\title{
Understanding the genetics and evolution of antimicrobial resistance in Escherichia coli
}

by

Kamya Bhatnagar

A thesis submitted to the Faculty of Graduate and Postdoctoral Affairs in partial fulfillment of the requirements

for the degree of

Doctor of Philosophy
in

Biology

Carleton University

Ottawa, Ontario

(C)2019

Kamya Bhatnagar 


\section{ABSTRACT}

Antimicrobial resistance (AMR) has emerged as a global public health challenge. The central goals of my $\mathrm{PhD}$ research are to better understand AMR evolution, with the longterm goal of developing novel strategies to mitigate the problem of AMR. This involves gaining comprehensive insights into the evolutionary trajectories of resistance evolution, investigation of the availability of resistance mutations and their pleiotropic consequences, and identification of genetic targets for the prevention/slowing of resistance evolution.

In a systematic study, I have investigated the effects of different chromosomal quinolone resistance mutations, and the pleiotropic effects of such mutations in Escherichia coli. I identified 50 spontaneous resistance mutants on nalidixic acid, ciprofloxacin, and levofloxacin, with mutations in regions of gyrA, gyrB, and marR, which are known contributors of resistance in quinolones. Resistant isolates had increased resistance levels, widespread cross-resistance to other quinolones, and overall significant costs of resistance in the absence of antibiotic. To investigate novel strategies to combat AMR, I carried out a small RNA screen on a quinolone resistant gyr background, to identify alternative drug targets. This screen led to the identification of 30 genes whose knockdown reduced the level of gyrase-mediated quinolone resistance in $E$. coli. Finally, to gain understanding of the network of genes contributing to intrinsic and phenotypic resistance, I have studied E. coli's two-component signal transduction systems (TCS). TCSs are involved in bacterial responses to many stresses, including 
antibiotics. I examined interactions between antibiotic stress and other environmental stressors in a set of eight TCS mutants. Mutants showed different types of responses to antibiotics and stressor+antibiotic combinations.

In this research, I have used novel approaches to understand bacterial resistance evolution and to combat AMR. On an applied level, these studies have implications for public health strategies. This research can help lead to better selection of appropriate antibiotics, alternative drug targets involved in resistance, and has prospects for development of new therapeutic approaches for combating AMR. 


\section{ACKNOWLEDGEMENTS}

First and foremost, I would like to express my sincere gratitude to my supervisor, Dr. Alex Wong, for introducing an inexperienced $\mathrm{PhD}$ student to the fascinating world of bacterial evolution and genetics. I am always amazed and constantly inspired to see your critical thinking, scientific abilities, wisdom, and compassion. Thank you, for providing me this opportunity to pursue my research. Through your kind, patient and supportive supervision, today I am a better researcher with broader perspectives and overall a new evolved version of myself. It was my great honour to pursue a $\mathrm{PhD}$ degree under your supervision, and an incredible journey that I will proudly remember.

I would like to thank my committee members, Dr. Ashkan Golshani and Dr. Thien-Fah Mah, who have been extremely helpful and guided me with valuable comments and feedback that broadened my research avenues.

I would like to acknowledge all the people that made my whole $\mathrm{PhD}$ journey memorable, and I am grateful to every one of them. I could not have enjoyed my experience at Carleton without the camaraderie of the Wong lab. I am privileged to have worked with and learned from such a fantastic group of scientists. Special thanks to my lab members - Ahlam, Prabh, Melissa, Trevor, Janelle, Tom, Gaby, Jess, Andrew, Nicole, Amanda, Bryn, Leah, Katie, and Aaron. I am appreciative of all the conferences, dinners, celebrations, parties, and other outside the lab fun activities. The lab 220 and my 
grad room 232 has been my home during all these years, and I will miss it dearly when I am gone.

Thank you, to my other Nesbitt friends - Chanchal, Gigi, Bhaswati, Brenda, Houman for being great support and for all the wonderful memories. I would also like to extend my appreciation to Nesbitt Biology staff at Carleton for all their support. Thank you, Laura, Lisa, Darlene, SarahAnne, Courtney, Ed, and Taye for making Nesbitt a happier place to work.

Thanks to my family, without whom I wouldn't be anywhere near where I am at now. To my husband, Mayank for all the support and for providing me with the unlimited supply of coffee just the way I like it. My little brother, Avichal for always supporting me. You are one person to whom I can speak my mind. Manda, thank you for being part of our family. You are such a beautiful soul. Last but not the least, Papa and Mummy. I cannot pen down anything to express my feelings of gratitude and appreciation for you both. I am here today, that is the fruit of your hard work, sacrifices, and blessings. Thank you for shaping me into the person I have become. Thank you for always supporting me. Thank you for everything! Always papa's little girl (:) 


\section{DEDICATION}

\section{This is for you - Manya}

$\sim$ The apple of my eye

सर्वं ज्ञानं मयि विद्यते (ऋग्वेद)

Sarv gyanam mayi vidyate (RigVed)

(All that I have to learn is within me) 


\section{STATEMENT OF CONTRIBUTION}

The thesis, "Understanding the genetics and evolution of antimicrobial resistance in Escherichia coli" is composed of three studies.

Chapter 2, "The mutational landscape of quinolone resistance in Escherichia coli", is the result of experiments primarily designed and carried out by myself with contributions from Dr. Alex Wong. Dr. Alex Wong contributed to the computational analysis of the whole-genome sequencing data. A version of this chapter is currently under review at PLOS One.

Chapter 3, "An sRNA screen for reversal of quinolone resistance in Escherichia coli", is the result of experiments primarily designed and carried out by myself with guidance from Dr. Alex Wong. Dr. Vandana Sharma and Dr. Yohei Yokobayashi (Okinawa Institute of Science and Technology) provided the small RNA expression vectors. Dr. Aaron Hinz made the allelic constructs for $\lambda$-Red recombinase technique. A version of this chapter is currently under review at G3.

Chapter 4, "Two-component regulatory networks as determinants of stress responses and antimicrobial resistance in Escherichia coli", is the result of experiments primarily designed and carried out by myself with contributions from Dr. Alex Wong. 


\section{TABLE OF CONTENTS}

$\begin{array}{lll}\text { Abstract } & \text { ii }\end{array}$

Acknowledgements $\quad$ iv

Dedication vi vi

Statement of contribution vii

Table of contents viii

List of Tables $\quad$ xiii

List of Illustrations $\quad$ xiv

List of Appendices $\quad$ xvii

List of Abbreviations $\quad$ xx

Chapter $1+1$

\section{General Introduction}

1.1 Antimicrobials and the evolution of antimicrobial resistance (AMR) 1

1.1.1 Challenges of antibiotic discovery 3

$\begin{array}{lll}\text { 1.2 Classification of Antibiotics } & 6\end{array}$

$\begin{array}{lll}\text { 1.2.1 Cell wall synthesis inhibitors } & 6\end{array}$

$\begin{array}{lll}\text { 1.2.2 Ribosomal inhibitors } & 7\end{array}$ 
1.2.4 DNA gyrase inhibitors $\quad 8$

1.2.5 RNA polymerase inhibitors 9

1.2.6 Membrane inhibitors 9

$\begin{array}{lll}1.3 & \text { Resistance mechanisms } & 11\end{array}$

$\begin{array}{lll}1.4 & \text { Fluoroquinolones } & 17\end{array}$

1.5 Persistence of AMR 26

$\begin{array}{lll}\text { 1.5.1 } & \text { Estimating fitness } 30\end{array}$

1.5.2 Implications for the control of AMR 30

1.6 Current investigations 31

$\begin{array}{ll}\text { Chapter } 2 & 34\end{array}$

The mutational landscape of quinolone resistance in Escherichia coli

$\begin{array}{lll}2.1 & \text { Abstract } & 34\end{array}$

$\begin{array}{lll}\text { 2.2 Introduction } & 35\end{array}$

2.2.1 Mutation 36

$\begin{array}{llr}\text { 2.2.2 } & \text { Selection } & 40\end{array}$

2.3 Materials and methods 44

2.3.1 Bacterial strains and media 44

2.3.2 Quinolone susceptibility assays 44

2.3.3 Fluctuation analysis and estimation of mutation rates 45

2.3.4 PCR amplification and sequencing of candidate genes 46 
2.3.5 Whole-genome sequencing 46

2.3.6 24-hour growth curve analysis $\quad 47$

$\begin{array}{lll}2.4 & \text { Results and discussion } & 48\end{array}$

2.4.1 Estimation of mutation rates 48

2.4.2 Identification of resistance mutations 50

2.4.3 Direct responses to selection 55

2.4.4 Cross-resistance between quinolones $\quad 57$

2.4.5 Cost of resistance $\quad 60$

2.5 Conclusions and Perspectives $\quad 64$

$\begin{array}{ll}\text { Chapter } 3 & 65\end{array}$

An sRNA screen for reversal of quinolone resistance in Escherichia coli

$\begin{array}{lll}3.1 & \text { Abstract } & 65\end{array}$

$\begin{array}{lll}3.2 & \text { Introduction } & 66\end{array}$

$\begin{array}{lll}\text { 3.3 } & \text { Materials and methods } & 70\end{array}$

3.3.1 Bacterial strains, media, and plasmid construction $\quad 70$

$\begin{array}{lll}\text { 3.3.2 Randomized library construction } & 71\end{array}$

3.3.3 Ciprofloxacin sensitivity screen $\quad 72$

$\begin{array}{lll}\text { 3.3.4 Target identification } & 73\end{array}$

$\begin{array}{lll}\text { 3.3.5 Validation for selected targets } & 74\end{array}$

$\begin{array}{lll}\text { 3.3.6 Ciprofloxacin susceptibility assay } & 75\end{array}$

$\begin{array}{lll}3.4 & \text { Results } & 76\end{array}$ 
3.4.1 Randomized sRNA library construction 76

$\begin{array}{ll}\text { 3.4.2 Ciprofloxacin sensitivity screening } & 79\end{array}$

3.4.3 Identification of target genes 83

3.4.4 Experimental validation of targets 83

$\begin{array}{llr}\text { 3.5 Discussion } & 88\end{array}$

$\begin{array}{lr}\text { Chapter } 4 & 90\end{array}$

Two-component regulatory networks as determinants of stress responses and antimicrobial resistance in Escherichia coli

$\begin{array}{lll}\text { 4.1 } & \text { Abstract } & 90\end{array}$

$\begin{array}{lll}\text { 4.2 } & \text { Introduction } & 92\end{array}$

$\begin{array}{lll}\text { 4.3 } & \text { Material and methods } & 98\end{array}$

$\begin{array}{llr}\text { 4.3.1 E. coli } \text { strains } & 98\end{array}$

$\begin{array}{lll}\text { 4.3.2 } & \text { Media and culture conditions } & 98\end{array}$

4.3.3 The growth of TCS mutant strains in M9 MM +/- stress conditions on solid medium

$\begin{array}{lll}\text { 4.3.4 24-hour growth curve analysis } & 100\end{array}$

$\begin{array}{ll}\text { 4.3.5 Antibiotic susceptibility assay } & 101\end{array}$

$\begin{array}{lll}\text { 4.4 Results and Discussion } & 102\end{array}$

4.4.1 The impact of stressors on TCS mutants 103

4.4.2 The impact of antibiotic on TCS mutants 104

4.4.3 The response of stressor+antibiotic combinations on TCS mutants 105 
$\begin{array}{lll}\text { 4.4.4 Antibiotic susceptibility } & 111\end{array}$

$\begin{array}{ll}\text { 4.5 Conclusions and Perspectives } & 116\end{array}$

Chapter 5

Concluding Remarks

Chapter 6

123

Future Perspectives

Literature cited

128

Appendices

206

A Appendix to Chapter 2

206

B Appendix to Chapter 3

213

C Appendix to Chapter 4

229 


\section{LIST OF TABLES}

Table 1.1 Different antibacterial mechanisms of action and their targets. $\quad 10$

Table 2.1 MIC fold-change and mutations in $\operatorname{gyr} A$, gyrB, and marR among $\mathrm{NAL}^{\mathrm{R}}, \mathrm{CIP}^{\mathrm{R}}$, and $\mathrm{LEVO}^{\mathrm{R}}$ mutants of E. coli K-12 (MG1655).

Table 2.2 Two-way analysis of variance (ANOVA) for the effects of antibiotic and gene on levels of resistance.

Table 2.3 Effects of resistance mutations on growth parameters.

Table 3.1 Randomized sequences of artificial sRNAs constructs.

Table 3.2 36 genes selected for experimental validation, encoding predicted sRNA targets associated with reductions in gyrA S83L mediated CIP resistance.

Table 4.1 A two-way analysis of variance (ANOVA) for the effects

of stressor and mutants.

Table 4.2 A two-way analysis of variance (ANOVA) for the effects of antibiotic and mutants.

Table 4.3 Effect of mutants on the growth rate (OD600/minute) parameter. 106 


\section{LIST OF ILLUSTRATIONS}

Figure 1.1 Mode of action of some commonly used antibiotics and their mechanisms

of resistance.

Figure 1.2 The quinolone core nucleus and the other structural modifications in the development of nalidixic acid, ciprofloxacin, and levofloxacin. $\quad 18$

Figure 1.3 Type II topoisomerases in E. coli. (A) DNA gyrase, and (B) topoisomerase IV.

Figure 1.4 Resistance mechanisms to FQ (CIP) in E. coli.

Figure 1.5 Schematic representation of MarRAB operon in E. coli.

Figure 2.1 The fluctuation assay.

Figure 2.2 Spontaneous mutation rate per $10^{8}$ cells to quinolone resistance among $E$. coli K-12 (MG1655).

Figure 2.3 The domain structures of gyrase A, B, and MarR.

Figure 2.4 Direct responses to selection. Changes in MIC for resistant mutants towards the drug on which they were selected: NAL (A), CIP (B), and $\operatorname{LEVO}(\mathrm{C})$. 
Figure 2.5 Cross-resistance between resistant mutants. Fold-increase in MIC of resistant mutants isolated on NAL (A), CIP (B), and LEVO (C) against all three antibiotics.

Figure 2.6 Cost of resistance of quinolone resistant mutants. The fitness components measured are growth rate, cell density, and lag time between gyrA, gyrB, and $\operatorname{mar} R$ resistance mutations.

Figure 2.7 No correlation between level of resistance (fold-change in MIC) and growth rate, cell density or length of lag phase for all mutants.

Figure 3.1 A schematic representation for the construction of the randomized artificial sRNA library.

Figure 3.2 Boxplot distributions of CIP-sensitivity phenotypes for $\sim 5000$ gyrA S83L mutants harbouring randomized sRNA plasmid constructs.

Figure 3.3 Effects of sRNA expression on growth rate ( $\mathrm{OD}_{600} /$ minute) and length of lag phase (minutes) with and without CIP.

Figure 3.4 Fold change in MIC values for 36 knockout mutants on wild-type and gyrA S83L backgrounds.

Figure 4.1 Schematic of a stereotypical two-component signal transduction system's (TCS) phosphorylation cascade. 
Figure 4.2 Growth rate of baeS and baeR mutants in the presence and absence of stressor $(\mathrm{NaCl})$.

Figure 4.3 Growth rate of $\operatorname{cpx} A$ and $\operatorname{cpx} R$ mutants in the presence and absence of stressor $(\mathrm{NaCl})$.

Figure 4.4 Growth rate of $p h o Q$ and phoP mutants in the presence and absence of stressor $\left(\mathrm{MgCl}_{2}\right)$.

Figure 4.5 Growth rate of $e n v Z$ and $o m p R$ mutants in the presence and absence of stressor $(\mathrm{NaCl})$.

Figure 4.6A $\log _{2} \mathrm{MIC}$ change of $p h o Q$, phoP TCS mutants under stressor $\left(\mathrm{MgCl}_{2}\right)+/-$ antibiotic conditions compared to control $c y b C$.

Figure 4.6B $\log _{2} \mathrm{MIC}$ change of envZ, ompR TCS mutants under stressor $(\mathrm{NaCl})+/-$ antibiotic conditions compared to control $c y b C$.

Figure 4.6C $\log _{2} \mathrm{MIC}$ change of cpxR, cpxA TCS mutants under stressor $(\mathrm{NaCl})+/-$ antibiotic conditions compared to control $c y b C$.

Figure 4.6D $\log _{2} \mathrm{MIC}$ change of baeR, baeS TCS mutants under stressor $(\mathrm{NaCl})+/-$ antibiotic conditions compared to control $c y b C$. 


\section{LIST OF APPENDICES}

Appendix - A

206

\section{Appendix to Chapter 2}

Table A2.1 Terminology used for the estimation of mutation rates. Based on MSS-

likelihood method.

206

Box A2.1 Equations used in for the estimation of mutation rates (Based on the MSS-

likelihood method).

207

Table A2.2 Spontaneous mutation rate, with $95 \%$ confidence interval in E. coli K-12 (MG1655) supplemented with different quinolone antibiotics. $\quad 208$

Table A2.3 MIC fold-change and resistance mutations in gyrA, gyrB and marR among $56 \mathrm{NAL}^{\mathrm{R}}, \mathrm{CIP}^{\mathrm{R}}$, and $\mathrm{LEVO}^{\mathrm{R}}$ mutants of E. coli K-12 (MG1655). 209

Figure A2.1 Comparison between mean fold-change in MIC values for different resistance mutations in $\operatorname{gyr} A, \operatorname{gyr} B$, and $\operatorname{marR}$ regions.

Figure A2.2 Variation in costs of resistance - growth rate, cell density, and lag phase between $\operatorname{gyr} A(\mathrm{~A}), \operatorname{gyr} B(\mathrm{~B})$, and $\operatorname{marR}(\mathrm{C})$ resistance mutations. $\quad 212$ 


\section{Appendix to Chapter 3}

Table B3.1 List of oligonucleotides used in this study.

Table B3.2 The list of preliminary (222) gene targets. All the gene targets identified from the 31 sRNA randomized sequences, and their putative mRNA complementarity hits.

Table B3.3 The preliminary 74 candidate genes based on their function among 31 artificial sequences of sRNA and their hybridization with target mRNA.

Table B3.4 MIC values $(\mathrm{ng} / \mathrm{ml})$ of single (Keio knockout mutant) and double mutant (Keio knockout mutant + gyrA S83L mutation) towards Ciprofloxacin.

Figure B3.1 A boxplot distribution of 528 randomized plasmid constructs growing in the presence and absence of CIP.

Figure B3.2 A model predicted by IntaRNA, showing the complementary base-pairing between artificial sRNA sequence and its target mRNA hit. 


\section{Appendix to Chapter 4}

Table C4.1 The list of stressors and their concentrations used in this study. 229

Table C4.2 The antibiotics used in this study and their mode of action.

Table C4.3 MIC values (ug/ml) of TCS mutants under stressor $+/$ - antibiotic conditions.

Table C4.4 Normalized colony size measurement for eight mutants compared to $c y b C$ on solid media under various experimental treatments.

Table C4.5 A two-way analysis of variance (ANOVA) for the effects of different treatments between control and mutants.

Figure C4.1 Structural features and domain organization of sensor (HK) and response regulator (RR) proteins of E. coli TCS.

Figure C4.2 Boxplot of averaged normalized colony-size scores comparing various growth treatments (M9 MM and stressor+/-antibiotic) of control $c y b C$ versus eight mutants.

Figure C4.3 A representative image for phenotypic quantification of colony size variation between control $c y b C$ and Keio knockout mutant env $Z$ under various treatments. 


\section{LIST OF ABBREVIATIONS}

\begin{tabular}{|c|c|}
\hline$\mu \mathrm{g}$ & Microgram \\
\hline$\mu l$ & Microlitre \\
\hline$\mu \mathrm{M}$ & Micromolar \\
\hline ATP & Adenosine triphosphate \\
\hline bp & Base pair \\
\hline $\mathrm{C}$ & Celsius \\
\hline DNA & Deoxyribonucleic acid \\
\hline g & Gram \\
\hline 1 & Litre \\
\hline M & Molar \\
\hline $\mathrm{mM}$ & Millimolar \\
\hline $\mathrm{mm}$ & Millimeter \\
\hline $\mathrm{ml}$ & Millilitre \\
\hline mRNA & Messenger RNA \\
\hline ng & Nanogram \\
\hline OD & Optical density \\
\hline PCR & Polymerase chain reaction \\
\hline RNA & Ribonucleic acid \\
\hline rpm & Revolutions per minute \\
\hline WT & Wild-type \\
\hline $\mathrm{w} / \mathrm{v}$ & Weight by volume \\
\hline
\end{tabular}




\section{Chapter 1}

\section{General Introduction}

\subsection{Antimicrobials and the evolution of antimicrobial resistance (AMR)}

With the breakthrough discovery of sulphonamides in the 1930's, followed by penicillin in the 1940's, antibiotics revolutionized medicine. During 1950's and 1960's, the “golden age" of antibiotic discovery saw the development of many new drug classes. The golden age was followed by the development of synthetic or semisynthetic derivatives of natural substances with improved spectrum and pharmacokinetic properties (Debabov 2013; Ventola 2015). Multiple classes of antibiotics were launched during this era, including the ß-lactam antibiotics, aminoglycosides, tetracyclines, macrolides, glycopeptides, polymyxins, and fluoroquinolones. Since 1990, pleuromutilins, lipoglycopeptides, oxazolidinones, and derivatives of older classes were launched. Antibiotic therapy made treatment of infections, surgery, transplantation, chemotherapy, neonatal care and prosthetic surgery safer and more efficient. 
However, soon the inevitability of antimicrobial resistance (AMR) evolved. AMR evolution is part of an ancient process of natural selection wherein organisms producing, and organisms targeted by antibiotics, have been in an arms race against each other (D'Costa et al. 2006; Wright and Poinar 2012; Andersson and Hughes 2017). As most antibiotics are natural products (Allen et al. 2010), resistance genes are already present in the producers, and thus are easily shared with other bacteria, including potential human pathogens (Davies and Davies 2010). Clinically relevant resistance appeared soon after the introduction of mass-produced antibiotics (Barber and Rozwadowska-Dowzenko 1948), with the first multi-drug resistant (MDR) enteric bacteria found within a few years (Watanabe 1963). Thereafter, there has been increasing use, overuse, and misuse of antibiotics in healthcare, agriculture, and livestock.

Major health organizations like the Centers for Disease Control (CDC), the World Health Organization (WHO) and the European Center for Disease Prevention and Control (ECDC) have recognized MDR as an emergent global crisis (Blair et al. 2014; Roca et al. 2015). In 2017, the WHO issued a list of "Priority Pathogens" categorized into critical, high, and medium priority for which new antibiotics are urgently required. 9/12 pathogens listed are Gram-negative bacteria, including all three critical priority pathogens (carbapenem-resistant Enterobacteriaceae (CRE), Acinetobacter baumannii, and Pseudomonas aeruginosa). The Canadian Antimicrobial Resistance Alliance (CARA 2015) reported the prevalence of AMR in a range of infectious pathogens among Canadian patients from 2007-2016 and presented the most common pathogens as Escherichia coli (23\%; resistant), Staphylococcus aureus (MRSA; 73\%), and $P$. aeruginosa (15\%). In addition to impacting public health, AMR is also burdening the 
economy. The World Bank has predicted that AMR will increase global healthcare costs by $\$ 300$ billion to $\$ 1$ trillion per year by 2050 (World Bank 2018).

\subsubsection{Challenges of antibiotic discovery}

Drug discovery has slowed down dramatically, leading to an innovation gap with a dry antibiotic development pipeline (Palumbi 2001; Levy and Marshall 2004; Spellberg et al. 2008; Fischbach and Walsh 2009; Andersson and Hughes 2010; Davies and Davies 2010; Silver 2011; Fair and Tor 2014; Boeckel et al. 2014; Ventola 2015). The reasons for the inadequate supply of new antibacterials are complex, but are in part due to the failure of industry and academia to identify new drug leads in the past 20 years (Payne et al. 2007; Gwynn et al. 2010; Livermore 2011).

All antibiotics developed during the golden age were discovered by empirical screening via inhibition of growth assays, and their mechanisms of action were determined much later (Silver 2011). In the 1990s, an alternative approach was pursued, focused on synthetic small molecules that bind to different essential targets. This knowledge led to a huge expansion of industrial efforts in which companies ran highthroughput screens (HTSs) on dozens of different targets. For example, GlaxoSmithKline (GSK) (1995-2001) ran 67 in vitro target-directed screens against over half a million compounds in search of potential antimicrobials. 16 of these screens resulted in hits, where a "hit" is a chemically tractable, low-micromolar potency inhibitor of the target with 10 times selectivity for bacterial over mammalian enzymes. Of these initial hits, only five were potent candidate leads (a "lead" has the qualities of "hit" as well as 
antibacterial activity due to inhibition of the in vitro targeted enzyme) with Gram-positive antibacterial activity. However, none of their sustained efforts generated successful new drug leads due to narrow spectrum of activity (Payne et al. 2007; Tommasi et al. 2015). At AstraZeneca (AZ), 65 HTS yielded 19 leads for Gram-positive infections, but no leads for Gram-negatives were identified (Tommasi et al. 2015). These screening approaches were profoundly unsuccessful for various reasons, including a lack of chemical diversity, the compounds were unable to cross Gram-negative bacterial cell walls, or a lack of correlation between activity in a biochemical assay and whole-cell activity. Such failures ultimately halted interest in novel antibacterials on the part of many major pharmaceutical companies.

Given the failure of HTS for antibiotic discovery, research strategies have turned to other novel methods to help bridge the innovation gap of antibiotic discovery. The use of whole-cell phenotypic screening approaches, for example, overcomes the limitation of a lack of translation of activity from biochemical assays, but this kind of screening requires follow-up mechanism of action studies. The screening of natural products can provide access to chemical diversity and it has unraveled known and new scaffolds (Cox et al. 2017). Thus, the search for novel stand-alone antibacterials with novel mechanisms of action continues to be an imperative measure to continue the fight against antibioticresistant bacterial infections. Challenges in drug discovery, along with accelerating drug resistance, mean that additional approaches should be explored.

Recent efforts have identified novel antibacterials using innovative technologies and conceptual approaches. With the help of novel culture methods, for example, the new 
compound teixobaction was discovered that inhibits cell wall synthesis in pathogenic bacteria, such as S. aureus and Mycobacterium tuberculosis (Piddock 2015; Ling et al. 2015). Other strategies include antibiotic adjuvants or antibiotic potentiators (as discussed in chapter 3 of this thesis) that can either block bacterial resistance mechanisms or enhance the antimicrobial action of a drug. The pursuit of antibiotic adjuvants helps to avoid the re-discovery of known drugs, and has the advantage that it lessens the continuous effort in the challenging and expensive identification of new targets that are essential for bacterial survival (Kalan and Wright 2011; Farha and Brown 2013; Gill et al. 2015; Brown 2015; Wright 2016; Melander and Melander 2017). Some examples of antibiotic adjuvants include clinically-approved $\beta$-lactamase inhibitors that restore the activity of ß-lactam antibiotics (Drawz et al. 2014). Inhibitors of aminoglycosidemodifying enzymes (AME) have also been developed, but none of them have been clinically approved (Ramirez and Tolmasky 2010; Labby and Garneau-Tsodikova 2013). Another set of potential adjuvant targets are the signaling and regulatory pathways used by bacteria to detect antibiotics and in turn activate resistance mechanisms. Some examples include inhibition of BlaR1 or MecR1 mediated identification of ß-lactam antibiotics in S. aureus (Boudreau et al. 2015), interference with the SOS DNA repair pathway (Alam et al. 2016), and interference with the two-component signaling system (TCS) pathways that have a role in antimicrobial intrinsic resistance or tolerance (as discussed in chapter 4 of this thesis) (Worthington and Melander 2013). 


\subsection{Classification of Antibiotics}

Antibiotics, of natural or synthetic origin, can be classified by various criteria, including their activity spectrum, origin, or mode of action. On the basis of spectrum, antibiotics can be active against a wide range of Gram-negative and Gram-positive bacteria (broadspectrum antibiotics), for example tetracyclines and chloramphenicol. Others have activity against limited range of bacterial species (narrow-spectrum antibiotics), for example macrolides and vancomycin (Campbell et al. 2001; Brandis et al. 2014). Antibiotics attack infections either by inhibiting growth of (bacteriostatic), or killing (bactericidal), the infecting bacteria. Antibiotics usually target essential bacterial pathways that are sufficiently different from or not present in human cells to avoid toxicity. The common targets of antibiotics are cell wall synthesis, protein synthesis, folate synthesis, DNA replication, and RNA transcription (Figure 1.1, Table 1.1).

\subsubsection{Cell wall synthesis inhibitors}

$\beta$-lactams are the largest group of antimicrobials used worldwide, and include penicillins, cephalosporins, carbapenems, and monobactams. These antibiotics are cell wall synthesis inhibitors and share a common chemical structure, the $\beta$-lactam ring. They target bacterial cell wall biosynthesis by binding and inactivating penicillin-binding proteins (PBPs) involved in cross-linking peptidoglycan (Walsh 2000; Poole 2004). They are commonly used to treat various bacterial infections caused by Gram-positive (penicillins) and -negative bacteria (cephalosporins, carbapenems, monobactams). 


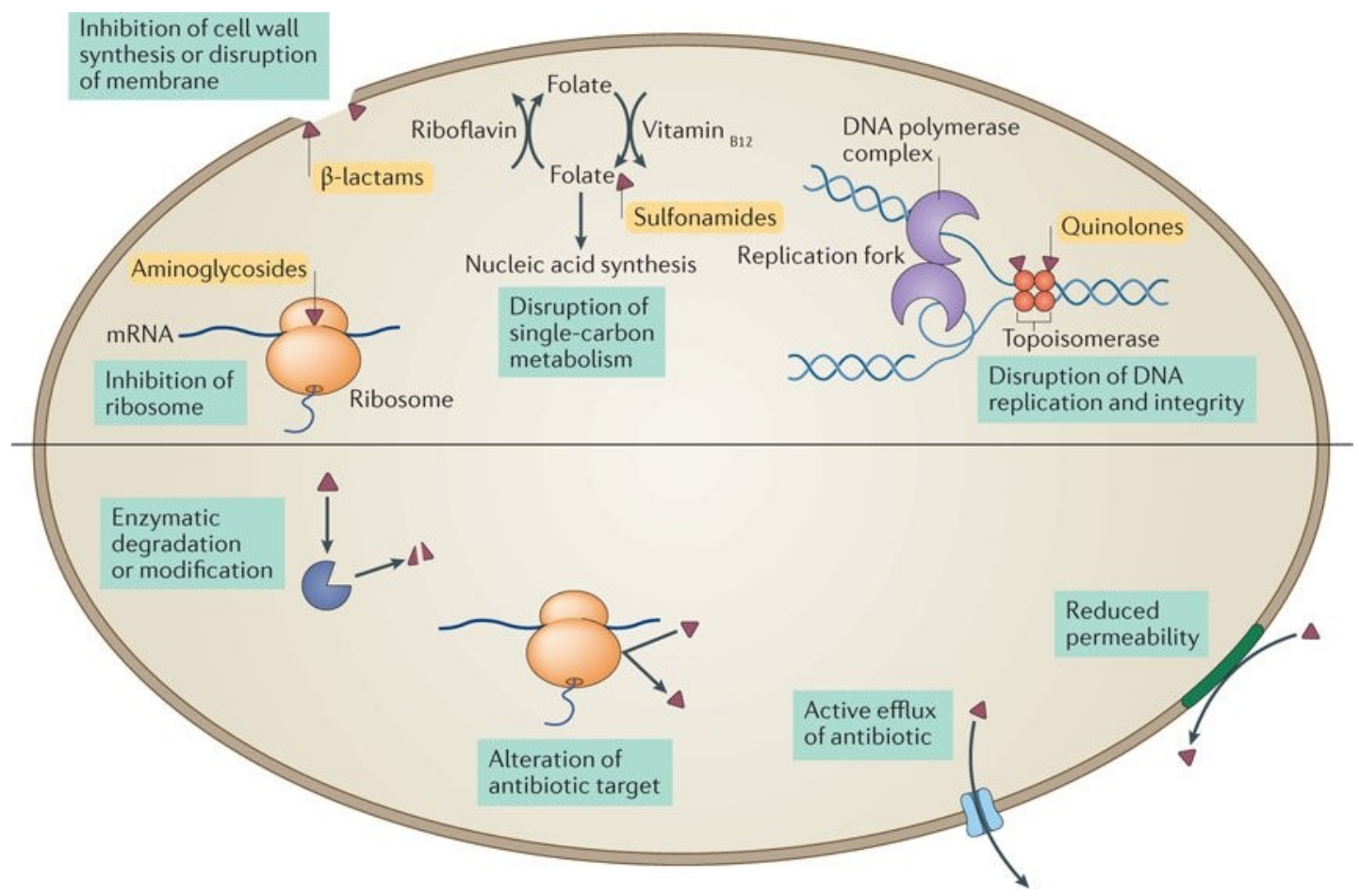

Figure 1.1 Mode of action of some commonly used antibiotics and their mechanisms of resistance (Crofts et al. 2017).

\subsubsection{Ribosomal inhibitors}

Some antibiotics target the ribosome and inhibit different stages of protein synthesis.

Some examples are aminoglycosides (such as kanamycin, amikacin, gentamicin, tobramycin, and streptomycin) that disrupt protein synthesis by binding to the $30 \mathrm{~S}$ subunit of the ribosome, blocking translation (Jana and Deb 2006). Tetracyclines are broad-spectrum, bacteriostatic antibiotics that bind to a different site on the $30 \mathrm{~S}$ ribosomal subunit and block elongation during translation. Macrolides are bacteriostatic antibiotics, mainly effective against Gram-positive bacteria. They bind to the polypeptide 
exit tunnel in the 50S subunit of the ribosome, thus blocking peptidyl transfer and protein elongation. Chloramphenicol is a broad-spectrum bacteriostatic antibiotic that binds to the 50S ribosomal subunit, inhibiting the peptidyl transferase of the ribosome (Walsh 2000).

\subsubsection{Folate synthesis inhibitors}

Bacteria are dependent on folate synthesis for production of nucleic acids, making the enzymes of this pathway targets for antibiotics. Sulfonamides and trimethoprim are important folate synthesis inhibitors, which inhibit the dihydropteroate synthetase (DHPS) and dihydrofolate reductase (DHFR) respectively. These antibiotics possess broad spectrum activity and are helpful in the treatment of urinary and respiratory tract infections (Hawser et al. 2006).

\subsubsection{DNA gyrase inhibitors}

Fluoroquinolones (FQs) are broad-spectrum antibiotics that bind to the type II topoisomerase enzymes DNA gyrase and topoisomerase IV (Topo IV). They are involved in the control of DNA supercoiling, transcription, and chromosome decatenation (Hooper 2001a; b). FQs stabilize the DNA-gyrase complex after cleavage of the DNA and thereby arrest DNA replication, transcription or decatenation, eventually leading to cell death (Ince and Hooper 2003). 


\subsubsection{RNA polymerase inhibitors}

Rifamycin antibiotics are a group of antibiotics that bind to the $\beta$-subunit of the bacterial RNA polymerase (RpoB), and sterically inhibit the elongation of the newly synthesized mRNA transcript (Conde and Lapa E Silva 2011). Rifampicin is the most popular broadspectrum bactericidal antibiotic of the rifamycin class and is used in the treatment of tuberculosis (Walsh 2003).

\subsubsection{Membrane inhibitors}

Antimicrobial peptides are considered as potential therapeutic agents as they are not commonly administered. The peptides often interact with the bacterial cytoplasmic cell membrane and disrupt the membrane (Hancock and Sahl 2006). Polymyxins are a class of cyclic peptides (such as colistin) that interact and disrupt the outer membrane (lipopolysaccharide) of Gram-negative bacteria, leading to increased permeability and cell death. 
Table 1.1 Different antibacterial mechanisms of action and their targets.

\begin{tabular}{|c|c|c|c|}
\hline Antibiotic class & $\begin{array}{l}\text { Molecular } \\
\text { target }\end{array}$ & Function inhibited & Examples \\
\hline ß-lactams & PBPs & Peptidoglycan synthesis & $\begin{array}{l}\text { Penicillin, Ampicillin, } \\
\text { Cephalosporins, Carbapenems }\end{array}$ \\
\hline Aminoglycosides & $\begin{array}{l}\text { rRNA of } 30 \mathrm{~S} \\
\text { ribosome subunits }\end{array}$ & Protein synthesis & $\begin{array}{l}\text { Gentamicin, Tobramycin, } \\
\text { Amikacin, Streptomycin, } \\
\text { Kanamycin }\end{array}$ \\
\hline $\begin{array}{l}\text { Trimethoprim, } \\
\text { Sulphonamides* }\end{array}$ & DHFR, DHPS & Folic acid synthesis & Trimethoprim, Sulfamethoxazole \\
\hline Quinolones & $\begin{array}{l}\text { Topoisomeraes } \\
\text { (DNA gyrase, } \\
\text { topoisomerase IV) }\end{array}$ & DNA replication & $\begin{array}{l}\text { Nalidixic acid, Fluoroquinolones: } \\
\text { Ciprofloxacin, Levofloxacin, } \\
\text { Moxifloxacin }\end{array}$ \\
\hline Macrolides & $\begin{array}{l}\text { rRNA of } 50 \mathrm{~S} \\
\text { ribosome subunit }\end{array}$ & Protein synthesis & $\begin{array}{l}\text { Erythromycin, Clarithromycin, } \\
\text { Azithromycin }\end{array}$ \\
\hline Tetracyclines & $\begin{array}{l}\text { rRNA of } 30 \mathrm{~S} \\
\text { ribosome subunit }\end{array}$ & Protein synthesis & Tetracycline \\
\hline Glycopeptides & $\begin{array}{l}\text { D-Ala-D-Ala of } \\
\text { lipid II }\end{array}$ & Peptidoglycan synthesis & Vancomycin, Teicoplanin \\
\hline Chloramphenicol & $\begin{array}{l}\text { rRNA of } 50 \mathrm{~S} \\
\text { ribosome subunit }\end{array}$ & Protein synthesis & Chloramphenicol \\
\hline Rifamycins & RNA polymerase & RNA synthesis & Rifampicin \\
\hline Polymyxins & Cell membrane & Disrupt cell membrane & Polymyxin B, Colistin \\
\hline
\end{tabular}




\subsection{Resistance mechanisms}

There are primarily two types of AMR, namely, intrinsic and acquired. Intrinsic resistance or innate resistance involves all of the inherent characteristics of a particular microorganism, such as physiological and genetic features, that contribute to its baseline resistance to antibiotics. Classically, intrinsic resistance is defined by the inherent capabilities of a bacterium to be resistant to certain antibiotics, due to impermeability of their cell wall, lack of target, or upregulated MDR efflux pumps (Nikaido 1989; 1994; Alekshun and Levy 2007; Fernández and Hancock 2012). Gram-negative bacteria, for example, are insensitive to many clinically effective antibiotics because many molecules cannot penetrate their double membrane cell wall structure (Cox and Wright 2013).

Studies of the intrinsic resistome have indicated a number of additional, non-conventional genes that can contribute to relative intrinsic resistance level and complement intrinsic resistance (Wright 2010; Cox et al. 2014). These genes fall under two categories of genes. The first category includes those genes whose inactivation can make bacteria more resistant to antibiotics, such as a mutation in a transcriptional repressor of an MDR efflux pump (Martinez and Baquero 2000; Martinez et al. 2007; 2011a; Fajardo et al. 2008).

The other set of elements are those whose inactivation make bacteria more susceptible to antibiotics. For example, in P. aeruginosa the inducibility of chromosomal ß-lactamase limits the efficacy of several ß-lactam antibiotics that are effective in other bacterial species (Martinez 2012). More recently, apart from the aforementioned classical resistance genes, several other elements from a variety of functional categories have been recognized to contribute to intrinsic resistance, including elements of bacterial general 
metabolism that respond to the presence of molecular signals, such as an antibiotics or another environmental stimuli (Fajardo and Martinez 2008; Fajardo et al. 2008; Girgis et al. 2009; Davies and Davies 2010; Sengupta et al. 2013).

One such metabolic situation where the bacterial intrinsic resistome is unveiled is when bacteria gain phenotypic resistance under specific growth conditions. Bacterial phenotypic resistance is a relatively unexplored area, and is a non-inherited, temporary survival mechanism for a bacterium to respond to surrounding environmental cues, e.g., stress, temperature, nutrient conditions, and antibiotics (Levin and Rozen 2006; Fernández and Hancock 2012; Olivares et al. 2013; Hughes and Andersson 2017). Bacteria display rapid metabolic downregulation or upregulation by alterations in their transcriptome, depending on the extracellular environmental conditions. Certain environmental stress responses in bacteria provide them with temporary tools to survive and increase the chances to resist antibiotic exposure, for example, tolerance to polymyxins or antimicrobial peptides can be mediated by low concentrations of magnesium or calcium (McPhee et al. 2003; Mulcahy et al. 2008). Therefore, phenotypic tolerance under metabolic control allow a certain proportion of the bacterial population to survive and become resistant despite the presence of antibiotics (Martinez and Rojo 2011).

Two-component signal transduction systems (TCSs) (chapter 4 of this thesis) constitute the most prevalent form of signal transduction used by bacteria to respond to changes in their surroundings. TCSs manage the repertoire of responses to fluctuating environmental conditions (Martinez et al. 2009). TCSs are involved in survival, 
physiology, virulence, and antibiotic resistance in pathogenic bacteria (Barrett et al. 1998; Stephenson and Hoch 2002; Rasko et al. 2008). TCSs include multistep phosphorelay cascades that are arranged in a complex array of networking circuits, which can detect and respond to different environmental stimuli and regulate large numbers of genes (Stock et al. 2000; Mascher et al. 2006; Gao and Stock 2009). TCSs in E. coli regulate the gene expression of many genes. For example, the EnvZ-OmpR TCS regulates expression of the outer membrane porin proteins $\mathrm{OmpC}$ and $\mathrm{OmpF}$ in response to osmotic stress and other environmental signals. While both $\mathrm{OmpC}$ and $\mathrm{OmpF}$ require OmpR for high-level expression, the extent to which each porin is expressed depends on the phosphorylation state of OmpR. At high-osmolarity conditions OmpR is phosphorylated by the sensor kinase EnvZ, resulting in increased transcription of ompC and repression of ompF (Aiba and Mizuno 1990; Cai and Inouye 2002). At lowosmolarity conditions, the expression pattern reverses. As porins serve as a gateway for many beneficial compounds to passively diffuse into the periplasm, small toxic compounds, including many antibiotics, such as ß-lactams and other hydrophilic antibiotics (tetracycline, chloramphenicol, and fluoroquinolones) can also use these channels to gain entry into the cell. Thus, a decrease in porin-mediated permeability caused by mutational inactivation of porins or alteration of the channel properties, can increase resistance to many different antibiotics, although often at a cost (Barrett et al. 1998; Nikaido 2003; Hirakawa et al. 2003; Fajardo et al. 2008; Bem et al. 2015). For example, removal of OmpC and $\mathrm{OmpF}$ in E. coli can cause a $20 \%$ reduction in exponential growth rate (Knopp and Andersson 2015). Similarly, in P. aeruginosa, the PhoP-PhoQ TCS affects susceptibility to antibiotics (aminoglycosides, antimicrobial 
peptides, and polymyxin B) as well as virulence by modifying the LPS layer (Macfarlane et al. 2000; Gooderham and Hancock 2009). Thus, investigating bacterial TCS and their contributions to intrinsic resistance will help to understand the capacity of bacteria to evade antibiotics, and may help to define novel targets whose inactivation can influence bacterial drug susceptibility.

In acquired resistance, a formerly susceptible microbe can become resistant either by incorporating new genetic material or as a result of mutations (Martinez et al. 2009). Acquired resistance is typically the primary concern when considering clinical breakthrough AMR. Acquired resistance can evolve through the accumulation of de novo mutations, typically in chromosomal genes, or via horizontal gene transfer (HGT) of resistance genes. Mutation driven resistance can render the bacterium resistant by modifying the antibiotic target, as is the case for FQ resistance mutations in $g y r A$, which codes for the A subunit of DNA gyrase (Bagel et al. 1999; Piddock 1999), or rifampicin resistance mutations in $r p o B$, which codes for the $\beta$ - subunit of RNA polymerase (Campbell et al. 2001). Resistance can also be achieved by reducing uptake; mutations that reduce expression of, or that alter the structure of membrane porins, cause resistance by this mechanism (for example, marR causing activation of efflux) (Nikaido 1989). Similarly, increased drug efflux can also lead to resistance - mutations leading to overexpression of the AcrAB-TolC efflux pump in E. coli confer resistance to FQs, phenicols, and some ß-lactams (Nikaido 1996; Komp-Lindgren et al. 2003). 
The general known acquired resistance mechanisms are:

1. Reduced influx of the antibiotic. Antibiotic molecules need to cross the cell envelope to reach sufficient intracellular concentrations. They can enter the cell via outer membrane porins (OMPs) but chromosomal mutations in the genes regulating OMPs (for example, OmpF in E. coli) can prevent or reduce the entry of the antibiotic into the cell (Nikaido 2003; Fernández and Hancock 2012). Resistance mediated by this mechanism is often less specific and effective against multiple antibiotics.

2. Increased efflux of the antibiotic. Bacterial MDR pumps transport various structurally unrelated compounds out of the cell. Amino acid alterations, deletions or insertions in genes (e.g. the mar operon and the AcrR repressor) regulating MDR pump expression (e.g. AcrAB-TolC pump in E. coli) can increase the efflux of antibiotics, such as ciprofloxacin, tetracycline and chloramphenicol out of the cell (Poole 2005). Some genetic changes can also lead to overexpression of the pump, increasing the level of resistance (Baucheron et al. 2004; Swick et al. 2011). In E. coli, the OmpF influx and AcrAB-TolC efflux systems are coregulated by the global transcriptional regulator MarA.

3. Target modification. The antibiotic target binding site can be altered by mutation, diminishing the affinity of the antibiotic towards the target. This resistance mechanism typically arises by chromosomal mutations. For example, mutations in the target encoding gene gyrA can increase resistance to ciprofloxacin (Heisig and Tschorny 1994; Piddock et al. 1999; Marcusson et al. 2009). In M. tuberculosis, a 
single point mutation in $r p o B$ causes rifampicin resistance (Garibyan et al. 2003; Lambert 2005).

4. Modification or inactivation of the antibiotic. Some bacteria produce antibiotic modifying enzymes that inactivate/modify antibiotics in ways that reduce their toxicity. The aminoglycoside-modifying enzymes can $\mathrm{N}$-acetylate (acetyltransferases), phosphorylate (phosphotransferases), or adenylate (nucleotidyltransferases) the aminoglycoside molecule and thus inactivate the antibiotic target binding (Smith and Baker 2002; Wright 2005). Resistance to ßlactams such as penicillin and cephalosporin is conferred by catalyzing the cleavage and inactivation of their ß-lactam ring mediated by B-lactamases (Poole 2004; Alekshun and Levy 2007).

Resistance by HGT often involves the acquisition of external genetic material that have evolved in other species and that are easily transferred between different organisms. The notable transferable mobile genetic elements consist of plasmids, transposons, insertion sequences, phages, and chromosome cassettes (Simillie et al. 2010; Stokes and Gillings 2011). In methicillin resistant S. aureus (MRSA), for example, the acquisition and expression of the mecA gene (coding the penicillin-binding protein-2a) on its chromosomal DNA spreads resistance to ß-lactam antibiotics. All MRSA strains contain

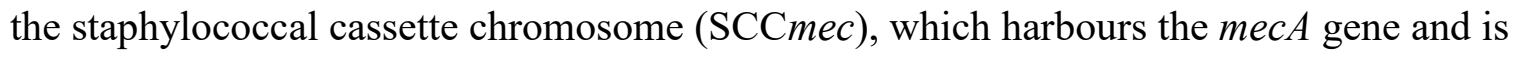
surrounded by mobile genetic elements Tn554 and IS431 (Malachowa and DeLeo 2010). The foreign piece of DNA can be acquired by the mechanisms of transformation (uptake of DNA from the surroundings), transduction (transfer of resistance genes via 
bacteriophage), or conjugation (transfer of plasmids with resistance genes from one bacterium to another via a pilus) (Ochman et al. 2000; Popa and Dagan 2011).

\subsection{Fluoroquinolones (FQs)}

The FQs are among the most important and frequently prescribed synthetic antibiotics. They have broad-spectrum activity, good pharmacokinetic and pharmacodynamic properties, and rapid bactericidal activity. Nalidixic acid (NAL) was the first quinolone to be discovered in 1962 as an impurity generated during the manufacturing of the antimalarial drug chloroquine, and was found to have antibacterial activity (Lesher et al. 1962; Mitscher 2005). NAL was initially used for the treatment of urinary tract infections (UTIs) caused by Gram-negative bacteria. However, limited clinical use, high toxicity, high minimum inhibitory concentration (MIC), and rapid resistance evolution spurred to the development of improved quinolones by modifications to the core nucleus (Emmerson and Jones 2003). Subsequently, in the 1970's, the additions of a 6-fluoro modification, and a 7-piperazine ring resulted in improved derivatives of NAL (Wolfson and Hooper 1985; Appelbaum and Hunter 2000) (Figure 1.2). The addition of fluorine increased drug penetration and drug target potency (Andersson and MacGowan 2003; Mitscher 2005). Following generations of FQs generated an improved spectrum of activity and pharmacokinetic properties. Examples include $2^{\text {nd }}$ generation ciprofloxacin (CIP), $3^{\text {rd }}$ generation levofloxacin (LEVO), and fourth-generation moxifloxacin (MOX). Some of the novel FQs currently in clinical trials, showing superior antimicrobial 
activity, include avarofloxacin, delafloxacin, finafloxacin, zabofloxacin, and nemonoxacin (Guan et al. 2013; Kocsis et al. 2016).

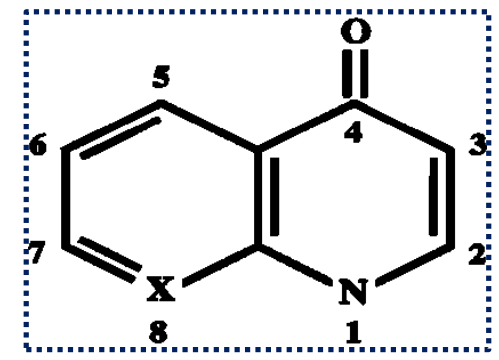

Quinolone core nucleus

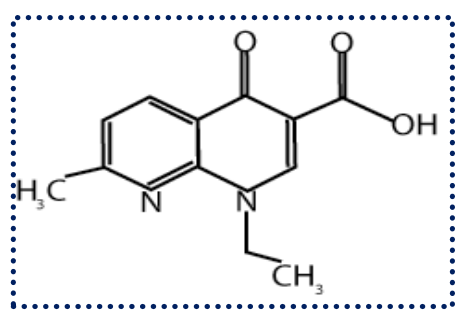

Nalidixic Acid

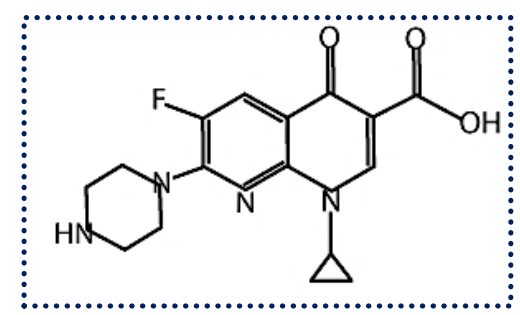

Ciprofloxacin

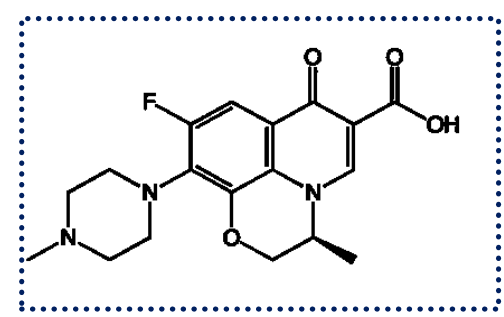

Levofloxacin

Figure 1.2 The quinolone core nucleus and the other structural modifications in the development of nalidixic acid, ciprofloxacin, and levofloxacin.

CIP, or 1-cyclopropyl-6-fluoro-1,4-dihydro-4-oxo-7-(1-piperazinyl)-3- quinolinecarboxylic acid, is a second-generation FQ. It is a clinically relevant antibiotic and is considered to be a highly prioritized important antimicrobial (WHO 2017). It is one of the most successful and widely used antibiotics with improved spectrum, bioavailability, and broad-spectrum activity against most Gram-negative (Enterobacteriaceae, 
Haemophilus influenza, Neisseria spp., P. aeruginosa) and some Gram-positive (S. aureus, Streptococcus pneumonia) bacterial pathogens. CIP is used to treat a wide range of bacterial infections including UTIs, gastrointestinal infections, respiratory tract infections, sexually transmitted diseases, and skin or bone related infections (Wolfson and Hooper 1985; Ball 2000; Guan et al. 2013).

FQs are DNA damaging agents that act by targeting two essential bacterial enzymes, DNA gyrase and DNA topoisomerase IV (Topo IV). Both target enzymes are type II topoisomerases that control the superhelicity of DNA to maintain a functional DNA structure (Figure 1.3) (Schoeffler and Berger 2008; Pommier et al. 2010). DNA gyrase is an ATP-dependent tetrameric protein composed of two GyrA and two GyrB subunits (A2B2), encoded by the genes gyrA and gyrB, respectively (Hooper and Jacoby 2015). DNA gyrase introduces negative, and relaxes positive supercoils generated by the separation of the strands during DNA replication ahead of the enzyme helicase. Gyrase catalyzes a double stranded break in the supercoiled DNA, passages another strand of the supercoiled DNA through it, and finally re-seals the broken DNA strands (Hooper 1999; Jacoby 2005). GyrA is involved in the double-strand breakage and reunion reaction, whereas GyrB is responsible for the ATPase activity of the enzyme. Topo IV is also a tetrameric protein $\left(\mathrm{C}_{2} \mathrm{E}_{2}\right)$, encoded by the genes parC and parE respectively. Topo IV relaxes both positive and negative supercoils and is primarily responsible for decatenation of interlinked daughter chromosomes at the end of replication process prior to cell division (Drlica and Zhao 1997; Hooper 2001; Schoeffler and Berger 2008; Pommier et al. 2010). 
A

DNA Gyrase
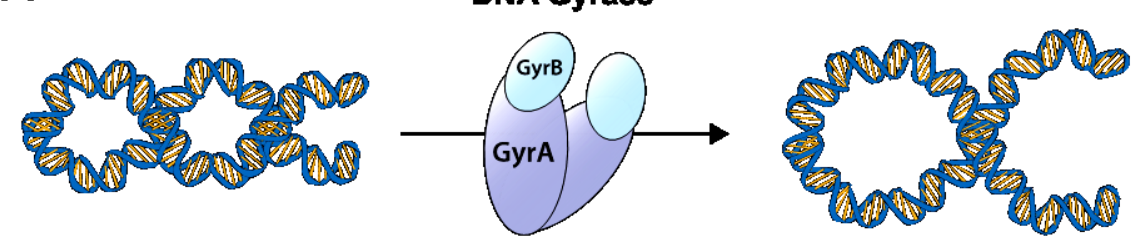

B
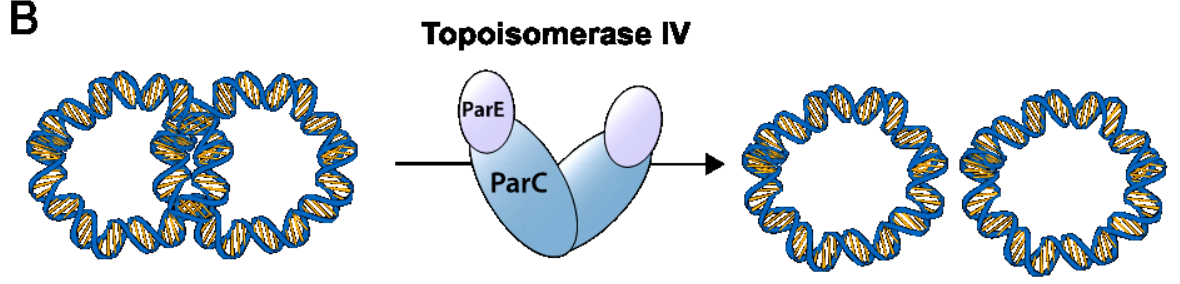

Figure 1.3 Type II topoisomerases in E. coli. (A) DNA gyrase (relaxes supercoils during DNA replication), and (B) topoisomerase IV (de-catenates daughter chromosomes before segregation process.

FQs form a complex with their target enzymes and the DNA in front of the replication fork. Type II topoisomerases bind to DNA crossovers and cleave one of the double-stranded DNA segments but remain attached to the 5' ends of the DNA so that the double-stranded DNA break is bridged. Subsequently, the enzyme associates with the intact double-stranded DNA segment and transfers it through the break, untangling the supercoil or catenation. Once the strand passage is completed, the double-stranded DNA break is re-ligated (Hooper 1999; Drlica et al. 2008; Schoeffler and Berger 2008). Consequently, the binding of FQs to the DNA-enzyme complex blocks the enzyme on the DNA and prevents resealing of the DNA strands, leading to the formation of lethal double-stranded DNA breaks (Drlica and Zhao 1997). The impasse created by FQs 
inhibits the movement of the replication fork and advancement of DNA polymerase resulting in cell death (Willmott et al. 1994; Hiasa et al. 1996).

The extensive use of FQs in clinical and veterinary medicine has resulted in the rapid emergence of resistance. Clinically relevant resistance to FQs is often due to the combined effects of multiple mutations. In E. coli, mutations are almost always present in gyrA. Additional mutations in $\operatorname{gyr} B$, $\operatorname{par} C$ and/or parE may be present, along with mutations in $\operatorname{mar} R, a c r R$ or $\operatorname{sox} R$ that alter efflux and influx. Thus, the complete resistome towards FQs in E. coli can be acquired through three main mechanisms: chromosomal mutations (single nucleotide substitution) that confer resistance to FQs by reducing drug-target binding, by reducing the intracellular concentration of the drug (by increased efflux), or downregulation of outer membrane porins (decreased influx through the membranes) (Figure 1.4) (Blair et al. 2014). 


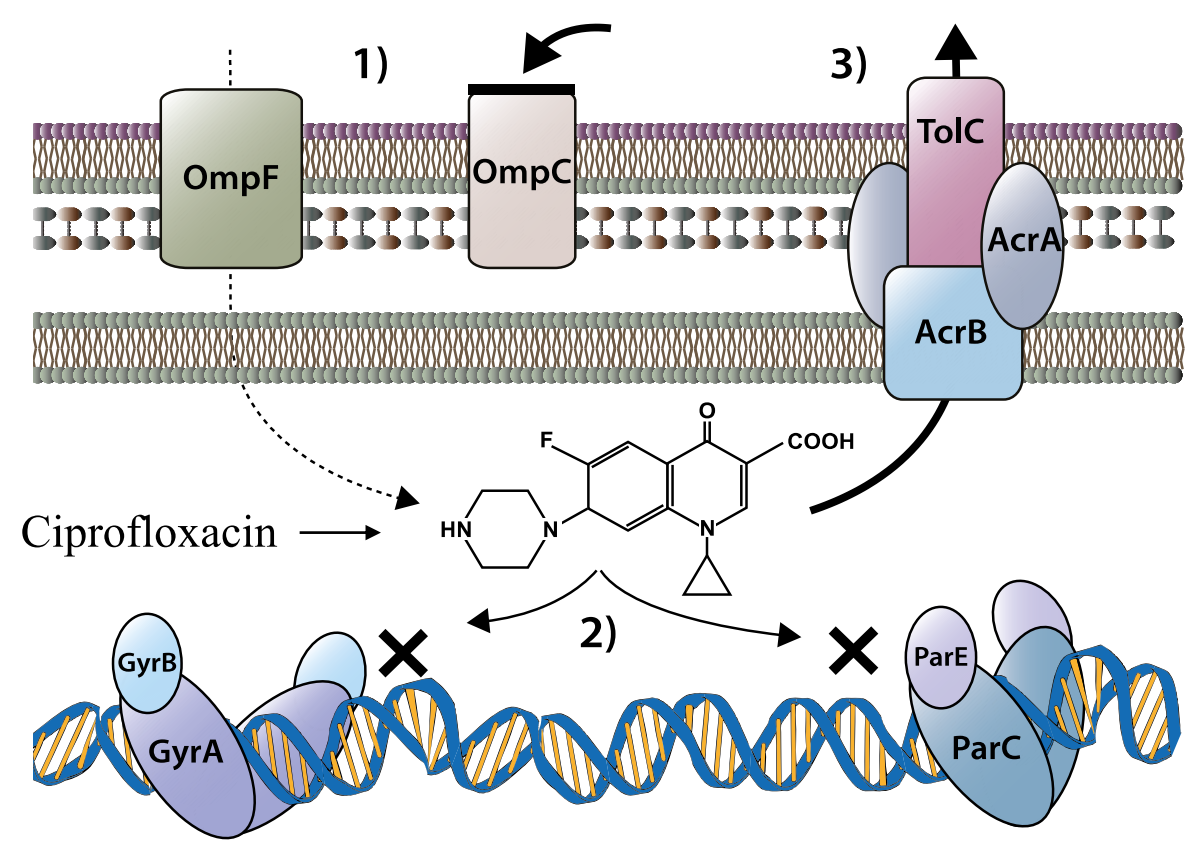

Figure 1.4 Resistance mechanisms to FQ (CIP) in E. coli involve: (1) downregulation of outer membrane proteins (OMP) (such as, porins $o m p F$ and $o m p C),(2)$ de novo mutations in DNA gyrase $(g y r A / B)$ and topoisomerase IV (parC/E), and (3) upregulation of AcrAB TolC efflux pump.

Mutations in the target genes gyrA, gyrB, parC, and parE often contribute to the $\mathrm{FQ}^{\mathrm{R}}$ phenotype (Deguchi et al. 1997; Komp Lindgren et al. 2003; Marcusson et al. 2009). The most frequently identified mutations in clinical isolates are located in a small part of gyrA (in particular amino acid residues S83 and D87) and parC (in particular amino acid residues S80 and E84), known as the quinolone resistance-determining region (QRDR). The QRDR corresponds to residues 67-106 of GyrA and residues 63-102 of ParC (Oram and Fisher 1991; Everett et al. 1996; Heisig 1996; Piddock 1999; Friedman et al. 2001; Komp Lindgren et al. 2003; Hopkins et al. 2005; Hooper and Jacoby 2015). 
Mutations in $g y r B$ are less frequent and the most studied are the D426N and K447E, displaying decreased susceptibility to NAL, CIP, and norfloxacin (Ince and Hooper 2003).

The most common mutations that appear frequently in clinically resistant isolates and increase levels of resistance above clinical breakpoints are combinations of gyr $A$ $\mathrm{S} 83 \mathrm{~L}, \operatorname{gyr} A \mathrm{D} 87 \mathrm{~N}, \operatorname{par} C \mathrm{~S} 80 \mathrm{I}$ and $\operatorname{parC} \mathrm{E} 84 \mathrm{~K} / \mathrm{A} / \mathrm{V} / \mathrm{G}$, and although less frequent, mutations in gyrB and parE are also observed (Vila et al. 1996; Drlica and Zhau 1997; Piddock 1999; Marcusson et al. 2009; Hooper and Jacoby 2015; Huseby et al. 2017; Basra et al. 2018). These combinations of mutations are associated with higher MIC gains, but reduced fitness costs, which possibly can explain their prevalence in clinical settings.

Mutations in $\mathrm{FQ}^{\mathrm{R}}$ clinical strains coding for protein repressors that regulate drug efflux are also often present. AcrAB-TolC is the major tripartite multidrug efflux pump associated with FQ resistance in E. coli (Alekshun and Levy 1997; Komp Lindgren et al. 2003; Marcusson et al. 2009; Li et al. 2015; Huseby et al. 2017). AcrAB-TolC is a multicomponent efflux transporter, the expression of which can be negatively regulated by the global regulatory genes of MarR, or the local repressors AcrR and SoxR (Komp Lindgren et al. 2003; Marcusson et al. 2009). Any inactivating mutation that downregulates the function of regulatory genes ( $\operatorname{mar} R, a c r R$, sox $R$ ) increases the expression of AcrABTolC, resulting in decreased intracellular concentrations of FQs and other antibiotics, such as chloramphenicol or tetracycline (Webber and Piddock 2003; Everett et al. 1996; Fernández and Hancock 2012). Efflux-related mutations cause a smaller gain in 
resistance phenotype than target mutations, but they hold a larger mutational target size. Notably, efflux mutations in combination with the target mutations can increase the MIC substantially (Marcusson et al. 2009). MarR is the repressor of the MarRAB operon and controls the intracellular level of the global transcriptional regulator MarA and MarB (Figure 1.5) (Alekshun and Levy 1997; Hooper 1999). MarR represses transcription of marRAB by binding as a dimer, within the mar operator, preventing binding of the RNA polymerase. MarR is a 144 amino acid protein folded into $\alpha$-helices and $\beta$-sheets and consists of a dimerization domain and a DNA-binding domain (Alekshun et al. 2001). The N- and C-terminal regions (amino acids 10-21 and 123-144) form the dimerization domain and intertwine with the corresponding regions in the other subunit of the dimer. The DNA-binding domain (amino acids 55-100) is composed of a winged-helix DNA binding domain ( $\alpha 2-\beta 1-\alpha 3-\alpha 4-\beta 2-W$ (wing)1- $\beta 3$ ) (Fig 2.2 of chapter 2 of this thesis).

The resistance gain associated with decreased porin production is small relative to other resistance mechanisms. This could be because FQs can also directly cross the outer membrane by diffusion through the phospholipid bilayer, and are thus not completely dependent on outer membrane porins for entry (Cohen et al. 1989; Nikaido and Thanassi 1993; Cramariuc et al. 2012). E. coli has of two major barrel-shaped outer membrane porins, OmpF and OmpC (Silver 2011; Li et al. 2015). CIP and other small hydrophilic molecules are able to enter through these channels. Therefore, the downregulation of porin expression due to mutations that reduce drug influx can also contribute to $\mathrm{FQ}$ resistance (Fernández and Hancock 2012). Inactivating mutations in marR (also discussed in chapter 2 of this thesis) can reduce the expression of the outer membrane porin $\mathrm{OmpF}$, causing a decrease in FQ influx. 


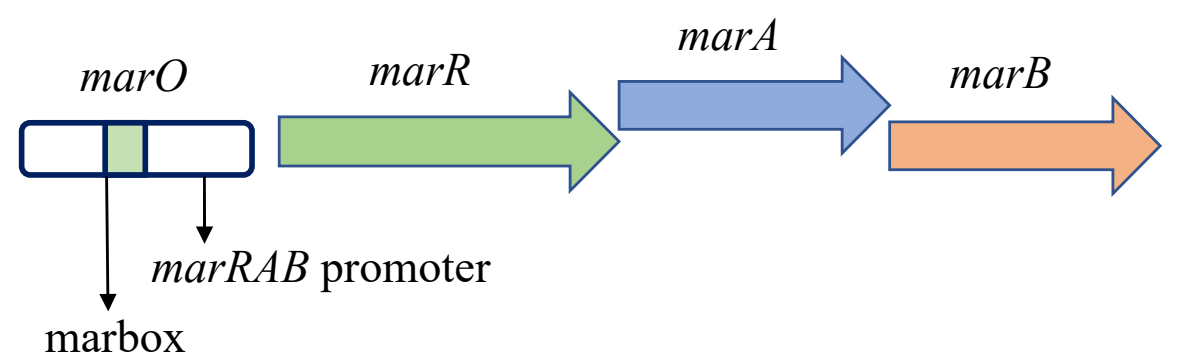

Figure 1.5 Schematic representation of MarRAB operon in E. coli.

Resistance to FQs is also associated with the presence of several genes acquired by HGT (Guan et al. 2013). These include plasmid-mediated quinolone resistance (PMQR) genes, which are now frequently reported in clinical isolates (Piddock 2014; Rodriguez-Martinez 2016). PMQR genes work by conferring target protection, drug modification, or drug efflux. Qnr proteins, a member of the pentapeptide repeat family, protect the target enzymes DNA gyrase and topoisomerase IV from FQ binding (Martínez-Martínez et al. 1998; Tran et al. 2005; Munita and Arias 2016). The efficacy of FQs is further reduced by the emergence of the plasmid-borne bifunctional aminoglycoside and FQ resistance gene $a a c-\left(6^{\prime}\right)-I b-c r$ (Ramirez and Tolmasky 2010). This gene, which classically encodes aminoglycoside $N$-acetyltransferase, differs by only two base pairs to modify FQs through acetylation. The acetylation reduces the antibacterial activity of FQs (specifically CIP), generating high MIC values (Robicsek et al. 2006a, b; Park et al. 2008). Recently, another CIP-modifying enzyme was found. A plasmid-borne gene named $\operatorname{crpP}$ isolated from a $P$. aeruginosa isolate increases CIP resistance by phosphorylation of the drug's carboxyl group (Chávez-Jacobo et al. 2018). 
A novel FQ inner membrane efflux pump QepA (quinolone efflux pump A) is flanked together with other antibiotic resistance determinants and through HGT can be expressed in the recipient cell (Périchon et al. 2007; Yamane et al. 2007). Several variants of QepA can be found in clinical isolates of E. coli: QepA2, QepA3, and QepA4 that differ from Qep1 by a few nucleotide changes. (Hooper and Jacoby 2015; Wang et al. 2017).

\subsection{Persistence of AMR}

The fitness costs or benefits associated with AMR mutations can influence the persistence, spread and emergence of antibiotic resistance within a bacterial population (Andersson and Levin 1999; Cohen et al. 2003; Andersson and Hughes 2010; Vogwill and MacLean 2015; Melnyk et al. 2015). In the presence of antibiotics, resistant mutants have an added fitness advantage over their susceptible counterparts. However, it is generally accepted that resistance is costly, and resistant bacteria suffer a metabolic burden in the absence of antibiotic that affects phenotypic traits, such as reductions in growth rate, survival, efficiency and stability relative to their sensitive genotypes (Andersson and Levin 1999; Reynolds 2000; Andersson 2006; Andersson and Hughes 2010). The fitness impairment is the repercussion of antibiotic mechanisms of action, since antibiotics target essential cellular enzymes in the bacterial cell and acquired resistance mutations often impair the processes mediated by these enzymes. For example, in $E$. coli, mutations in the RNA-polymerase $\beta$-subunit encoded by $r p o B$, which is the target of rifampicin (RIF), can confer a high-level of resistance but reduced transcriptional efficiency and eventually the growth rate (Reynolds 2000; O'Neill 2006; 
Brandis and Hughes 2013). Likewise, mutations in the target of streptomycin (STR), i.e. the ribosomal protein $\mathrm{S} 12$ encoded by $r p s L$, can have adverse effects in the form of decreased translational elongation rates in E. coli and S. typhimurium (Björkman et al. 1999; Levin et al. 2000). Some other examples where target alteration mutations confer a reduction in biological fitness are fusidic acid resistance caused by fusA mutations (Macvanin et al. 2003; 2004) or accumulation of FQ resistance mutations in DNA gyrase (Komp Lindgren et al. 2005; Marcusson et al. 2009). Similarly, resistance conferred by efflux-regulating mutations and porin-deficient mutants (OmpC and $\mathrm{OmpF}$ in E. coli) are also associated with a disorder in bacterial physiology, such as reduction in the exponential growth rates or reduced virulence in case of pathogenic organism (Andersson and Levin 1999; Maisnier-Patin and Andersson 2004; Du Toit 2017; Olivares et al. 2017).

Naively, it would be anticipated that scaling back of antibiotic selective pressure will favor selection against resistance, raising the possibility of reduction in resistance frequencies among bacterial population. But not all resistance mutations bear a cost, and such low, or no cost mutations are likely to persist and provide comparable fitness levels to resistant bacteria as their susceptible counterparts in the absence of antibiotic selection pressure (Björkman et al. 2000; Gottesman et al. 2009; Enne et al. 2010; Andersson and Hughes 2010; Sundqvist et al. 2010). For example, some $\mathrm{RIF}^{\mathrm{R}} r p o B$ and $\mathrm{STR}^{\mathrm{R}} r p s L$ mutations do not impose a fitness cost (Marcusson et al. 2009; Brandis et al. 2014).

Resistant clones that suffer a cost also often restore their fitness, and thus are not rapidly outcompeted by susceptible strains in antibiotic-free environments. This can 
occur via a genetic reversion to the wild-type state, which is unlikely since only one single nucleotide polymorphism (SNP) will cause the reversion and that will always cause loss of resistance. A more likely consequence is that resistant mutants acquire additional fitness-compensatory mutations while still maintaining resistance in the population (Handel et al. 2006; Andersson and Hughes 2010). The compensatory mutations have a larger mutational target size than reversion and can occur in the same gene that causes the resistance phenotype (intragenic compensation), or in other genes (extragenic compensation) causing a restoration of the functionality of the protein targeted by the antibiotic, and stabilizing the resistant mutants in the population (Schrag et al. 1997; Björkman et al. 2000; Reynolds 2000; Nagaev et al.2001; Maisnier-Patin and Andersson 2004; Marcusson et al. 2009; Hall et al., 2010; Andersson and Hughes 2010). For example, low-fitness $\mathrm{STR}^{\mathrm{R}}$ rps $L$ mutants compensate the deleterious effects of protein elongation rates by intragenic compensatory mutations, as well as via mutations in $r p s D$ or rpsE (Schrag et al. 1997; Björkman et al. 1998; 1999). Likewise, the fitness defects caused by rpoB mutations in $\mathrm{RIF}^{\mathrm{R}}$ mutants of Mycobacterium and Salmonella are ameliorated by second-site mutations in $r p o B$ itself (Reynolds 2000), or by other mutations in rpoA, or rpoC (Comas et al. 2011). Studies have also shown that strains having multiple resistance mutations are fitter than expected given the fitness of strains that carry only one mutation (Trindale et al. 2009; Borrell et al. 2013; Durão et al. 2015; Vogwill et al. 2016).

Some other factors can also influence bacterial fitness while still maintaining resistance, such as epistasis interactions among different resistance elements (Weinreich et al. 2006; Rozen et al. 2007; Trindade et al. 2009; Silva et al. 2011; Wong 2017), or 
selection of resistance mutations by unrelated stressors, such as the environment or genetic background (Trindade et al. 2009; 2012; Rodríguez-Verdugo et al. 2013). Epistasis, or genetic interactions, refers to the influence of genetic background on the fitness effect of a mutation. Thus, the effect of a mutation in a gene can vary depending on the presence/absence of mutations elsewhere in the genome (Cordell 2002; Weinrich et al. 2005; Lehner 2011). AMR mutations and associated compensatory mutations may interact epistatically, as compensatory mutations can be beneficial in the presence of AMR mutation but not otherwise. For example, reduced growth rate due to an increase in the elongation time among $\mathrm{STR}^{\mathrm{R}}$ mutations in $r p s L$ can be restored by compensatory mutations in rpsL. However, in a wild-type background, both resistant and compensatory mutations could be costly (Maisnier-Patin et al. 2002; Sander et al. 2002). In addition, the phenotypic effect of a mutation depends on the genetic context or strain in which it has appeared. For example, the fitness effects of rpoB mutations in different strains of Pseudomonas exhibit strain-dependent fitness effects (Vogwill et al. 2016). Moreover, some studies (Rozen et al. 2007; Trindade et al. 2009; Silva et al. 2011) have also demonstrated pervasive epistasis via in vitro competition assays in the absence of antibiotics, where the same resistance allele may have different selective effects across genetic backgrounds. 


\subsubsection{Estimating fitness}

Due to importance of fitness in determining the persistence of resistance, there is substantial interest in estimating the fitness effects of resistance mutations. Fitness can be measured in several ways, by comparing the relative maximum exponential growth rate, whereby a strain with reduced doubling time compared to another strain will be considered less fit. Doubling times are calculated using optical density measurements of a growing culture. The present studies in this thesis use exponential growth rate as a proxy for bacterial fitness. Competition assays are another method for fitness estimation, whereby two or more isogenically related susceptible and resistant strains are co-cultured and a fraction of the culture is transferred into fresh medium periodically. Using different genetic markers, the relative frequencies of the strains are quantified at set time.

Phenotypic traits can also be used as fitness proxies. These assays assess the strain's capability to withstand different stresses, such as salt, heat, osmolarity or acid conditions (Nagaev et al. 2001; Paulander et al. 2009; Hall et al. 2011).

\subsubsection{Implications for the control of AMR}

The economic and human cost of AMR is accelerating uncontrollably worldwide. To control the global unwanted impact of AMR, aggressive efforts are urgently required, such as improving global surveillance data of AMR, finding new antibiotics, reducing antibiotic use in food production, and facilitating research trials. In recent times, the WHO (WHO report 2017) has recommended scaling down the unnecessary use of 
antibiotics in animals. This step will help to preserve the effectiveness of medically important antibiotics in food producing animals and to control the emergence of resistance. Interestingly, as investigated by Enne et al. 2010, efforts to reduce the prevalence of resistance by restricting the antibiotic use in both animal and human populations has had mixed outcomes. In some cases, reduced antibiotic prescriptions lead to reduced prevalence of resistance but not in others. For example, in S. pneumoniae the prevalence was reduced after reduction of penicillin, however a persistent quinolone resistant E. coli was found remotely where quinolones were not prescribed (Austin et al. 1999; Enne et al. 2001; Enne 2010; Pallecchi et al. 2012). On a similar note, a recent systematic review and meta-analysis by Tang et al. (2017) found that interventions that restrict the use of antibiotics in food-producing animals can reduce the prevalence of AMR bacteria by 39\%. Such policy decisions that harmonize regulation will certainly help positively in restricting AMR and should be the focus of future research. Overall, these strategies assume that resistance is costly, which is supported by the results obtained in chapter 2 of this thesis.

\subsection{Current investigations}

While the primary mechanisms of mutation-driven resistance are understood for most drugs, there are important research gaps in understanding the full spectrum of resistance mutations and their pleiotropic effects even for well-studied antibiotics. Thus, I have begun my thesis with the aim of understanding the rate and consequences of chromosomal resistance mutations in order to investigate their evolutionary fates. 
Chapter 2 is the first study that attempts to generate a full catalogue of quinolone chromosomal resistance mutations and their pleiotropic effects. Quinolones are an important synthetic class of antibiotics that are used widely in clinical treatments. I have identified a number of resistance mutations in regions of the known contributors of quinolone resistance (DNA gyrase subunits A and B, and the MDR regulator Mar). I observed variation in resistance levels between mutations, widespread cross-resistance between quinolones, significant costs of resistance among resistant isolates. These findings have implications for public health strategies, chiefly in the context of antibiotic selection.

Chapter 3 is an attempt to understand the genetic network underpinning the function of DNA gyrase, and to take advantage of novel vulnerabilities in quinolone resistant gyrA S83L mutants. Using a synthetic genetic small RNA screen, I have identified genes whose inhibition increases susceptibility to quinolones in gyrA $\mathrm{S} 83 \mathrm{~L}$ mutants of E. coli. These genes are thus potential targets for restoring quinolone susceptibility in the clinic, either through the use of sRNA or small molecule inhibitors. More broadly, I show that sRNA screens can be a powerful tool for investigating genetic networks in bacteria.

While high-level acquired resistance can result from one or a few mutations, a broader network underlies intrinsic resistance. Intrinsic resistance in part results from the impermeability of the bacterial cell wall - for example, the outer membrane of Gramnegative bacteria serves as a permeability barrier to many antibiotics, and constitutive expression of membrane spanning efflux pumps can reduce the intracellular antibiotic 
concentration to sub-inhibitory levels (Cox and Wright 2013). In other cases, intrinsic resistance can result from the lack of a particular drug target, or from the formation of biofilms or spores. In the last decade, genome-scale screens have identified many additional redundant constellations of genes and proteins that contribute to intrinsic resistance to antibiotics. For example, the analysis of comprehensive mutant libraries from E. coli (Tamae et al. 2008; Liu et al. 2010) and P. aeruginosa (Breidenstein et al. 2008; Fajardo et al. 2008) has demonstrated that many genes contribute to intrinsic resistance. Two-component signal transduction systems (TCSs), which are involved in bacterial responses to many stresses, have emerged as important players in intrinsic resistance.

In Chapter 4, I examine the involvement of E. coli's TCSs in responding to multiple stresses. To do so, I investigate the impact of multiple stressors, including antibiotics, on eight TCS mutants of $E$. coli. I examine the response of each mutant to a variety of environmental stress conditions in combination with four antibiotics from different classes. This study broadens our understanding of the mechanisms by which $E$. coli's responds to external stressors, including antibiotics.

Chapter 5 is the concluding chapter that briefly summarizes the principal findings of this thesis. I finish with Chapter 6, discussing the future perspectives in light of prevalence of AMR . 


\section{Chapter 2}

\section{The mutational landscape of quinolone resistance in}

\section{Escherichia coli}

\subsection{Abstract}

The evolution of antibiotic resistance is influenced by a variety of factors, including the availability of resistance mutations, and the pleiotropic effects of such mutations. Here, we isolate and characterize chromosomal quinolone resistance mutations in Escherichia coli, in order to gain a systematic understanding of the rate and consequences of resistance to this important class of drugs. We isolated over fifty spontaneous resistance mutants on nalidixic acid, ciprofloxacin, and levofloxacin. This set of mutants includes known resistance mutations in $\operatorname{gyr} A$, gyrB, and $\operatorname{mar} R$, as well as two novel $g y r B$ mutations. We find that, for most mutations, resistance tends to be higher to nalidixic acid than relative to the other two drugs. Resistance mutations had deleterious impacts on one or more growth parameters, suggesting that quinolone resistance mutations are generally costly. Our findings suggest that the prevalence of specific gyrA alleles amongst clinical isolates are driven by high levels of resistance, at no more cost than other resistance alleles. 


\subsection{Introduction}

The increasing prevalence of antimicrobial resistance (AMR) has become an urgent public health problem worldwide. For example, Escherichia coli resistance to ciprofloxacin, the antimicrobial most purchased by hospitals in Canada between 20082014 (PHAC 2015-2016), rose to $26.7 \%$ in 2015 from $21.6 \%$ in 2009 (CARA 2015). The present AMR crisis has been attributed to the misuse and overuse of antibiotics, as well as the scarcity of novel drug development (Piddock 2012; Bartlett et al. 2013; Gross 2013; Gould and Bal 2013; Viswanathan 2014). Given the rapid increase in the prevalence of resistance, an understanding of the principles underlying resistance evolution is vital.

Adaptation, of which the evolution of AMR is a prime example, is driven by the interplay between mutation, selection, and demographic processes like drift. Mutation determines the rate at which beneficial variants are introduced into a population, while selection and demography govern the fates of these variants. Thus, in understanding the evolution of AMR, we are interested in both mutation and selection. We expect, for example, that higher mutation rates will generally lead to a more rapid evolution of resistance. The spread of a given mutation will then be influenced by its selective consequences, including its effect on resistance, and on its pleiotropic effects, such as fitness in the absence of antibiotic, and collateral sensitivity or cross resistance to other antibiotics. 


\subsubsection{Mutation}

The rate of mutation to resistance is given by the product of population size $(N)$, overall mutation rate $(\mu)$, and the fraction $(f)$ of mutations that are beneficial (i.e., those that grant resistance). Thus, resistance mutations will appear more frequently for larger populations, or for populations with higher mutation rates. Up to a point, this dependence on $N \mu$ leads to an increase in the rate of adaptation; however, as $N \mu$ approaches 1, a population is no longer limited by mutational input, but instead by competition between competing mutations (e.g., Gerrish and Lenski 1998; Wilke 2004).

Relevantly here, the fraction of mutations granting resistance almost certainly differs between different antibiotics; for some antibiotics, there will be a greater availability of resistance mutations. Variation in the availability of resistance mutations may reflect differences in the number of genetic loci that can contribute to resistance. For example, resistance to trimethoprim is largely conferred by mutations in a single gene encoding dihydrofolate reductase (DHFR) (Toprak et al. 2011), while resistance to chloramphenicol and streptomycin can be conferred by mutations in a number of different genes (Toprak et al. 2011; Winkler and Kao 2012). Moreover, the number of individual mutations conferring resistance may differ between genes: while a wide range of loss-of-function lesions in the transcriptional regulator $\operatorname{mar} R$ will grant multi-drug resistance (Alekshun et al. 2000), only a handful of mutations in gyrA confer quinolone resistance (Ruiz 2003; Hooper 1995). Thus, even given equal population size and overall mutation rates, we expect different rates of evolution to resistance to different antibiotics due to differences in the mutational target size. 
At a population level, mutation can be quantified by mutation rates or the mutation frequencies. The mutation rate is an estimate of the probability of a mutation occurring in an organism or gene during each generation or per cell division, whereas mutation frequency is simply the proportion of mutant bacteria present in a culture. The number of mutated cells in a culture depends on when the mutation occurred during the growth of the bacterial population. If a mutation occurs early in the growth of the culture, the number of mutants will be higher than those that arise later during growth. These parameters cause the frequency to vary greatly, making the frequency method an inaccurate measurement of mutation. As such, mutation rate is the preferable parameter to estimate (Rosche and Foster 2000).

A number of methods have been developed for calculating the rate of mutation; all of these methods measure the probable number of mutations per generation in a culture (Rosche and Foster 2000). The two broadly used methods are mutation accumulation assays and fluctuation assays. In a mutation accumulation assay the population is propagated through recurrent bottlenecks, presumably of a single cell, such that all mutations are nearly neutral, and the effect of selection is mitigated. Sequencing can then be used to quantify the number of mutations accumulated (Kibota and Lynch 1996; Garcia-Dorado and Gallego 2003).

Fluctuation assays are based on a model of clonal expansion of mutant clones as described by Luria and Delbrück (1943) from bacteriophage T1 sensitivity to resistance. In this method, the distribution of number of mutants is determined in parallel cultures and mutation rate is estimated from this distribution (Figure 2.1). Luria and Delbrück 
calculated the mutation rates of $E$. coli from a phage sensitive phenotype to a phage resistant phenotype. E. coli cultures exposed to the bacteriophage become clear at first because of cell lysis, but become turbid after prolonged incubation, since some T1 phageresistant cells would survive. $\mathrm{T} 1^{\mathrm{R}}$ mutations occurring early in the experiment, prior to the exposure of the phage, would be present in higher frequencies than $T 1^{\mathrm{R}}$ variants arising during viral exposure. Therefore, the frequencies of the resistant variants differ between independent cultures, depending on when during growth the mutations have taken place. As the number of mutant cells per culture varies due to the timing of mutation events, Luria and Delbrück argued that the distribution of mutant colonies did not follow a Poisson distribution and resistant mutants arise independently in the culture, even before selection on bacteriophage. However, the number of mutations that arise in each culture per generation follows a Poisson distribution. 

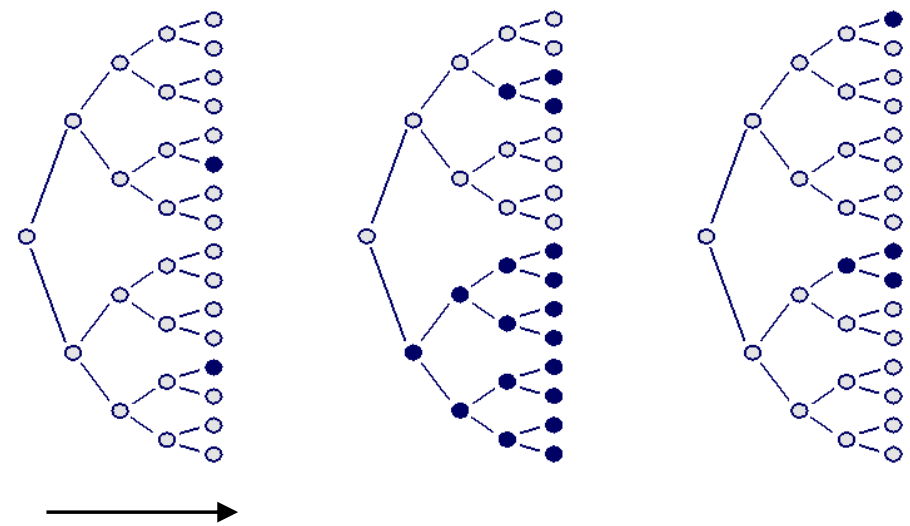

Generations

Figure 2.1 The fluctuation assay. Each of the three cultures has two mutational events during growth; however, the number of mutant cells varies depending on when during growth the mutations have taken place.

Several statistical methods have been developed for analyzing the results of fluctuation assays. Fluctuation assays provide the distribution of the numbers of mutants per culture, $r$, which is used to calculate $m$, the number of mutations per culture. When $m$ is divided by number of cells, $\mathrm{N}_{\mathrm{t}}$, it provides the mutation rate $\mu$. Although the distribution of mutants is not Poisson, the distribution of the number of mutations is a Poisson parameter. Many methods are used to calculate $m$, each having its pros and cons, depending on the particular experimental conditions. The $p_{0}$ method is suitable when the number of mutational events in a culture is low; this method estimates the mutation rate from the fraction of plates on which no colonies are observed, and thus does not use all of the data. The classical Lea and Coulson (LC) (1949) is the method of the median. This method assumes that if the median number of mutants is large enough, then most 
mutations occur earlier. The major drawbacks of this method are that it uses little information from the fluctuation assay, and it is sensitive to any variation in the assumptions, e.g., phenotypic lag, which will result in reduced precision. The most accurate method for estimating $m$ is the Ma-Sandri-Sarkar maximum likelihood method (MSS-MLE), which has become the standard method for calculating mutation rates. This method is valid for any range of the expected number of $m$, and $95 \%$ confidence intervals can be measured by an empirically determined set of equations. Moreover, it also calculates mutation rate from the entire dataset unlike the LC and $p_{0}$ methods (Ma et al. 1992; Rosche and Foster 2000). Mutation rate per cell is then calculated as $\mu=m / \mathrm{N}$, where $\mathrm{N}$ is the mean number of cells per culture, or approximately the number of cell divisions per culture (Sarkar et al. 1992; Hall et al. 2009).

\subsubsection{Selection}

Once a resistance mutation has arisen in a natural population, its persistence and spread may be affected by its pleiotropic effects, including its fitness costs and effects on resistance to other drugs. (Andersson and Levin 1999; Andersson and MacGowan 2003; Pál et al. 2015; Vogwill and MacLean 2015). In the presence of antibiotic, a resistant bacterium has a clear advantage compared to susceptible genotypes. However, in an antibiotic-free environment, a resistance mutation may impose a burden, for example through reduced growth rates relative to sensitive strains (Reynolds 2000; Levin et al. 2000; Tenover 2006; Hall et al. 2011). However, while resistance mutations are often costly, not all resistance mutations bear a cost, and such cost-free mutations are likely to 
persist (Komp-Lindgren et al. 2003; Luo et al. 2005; Zhang et al. 2006; Rozen et al. 2007). Melnyk et al. (2015) conducted a meta-analysis including 179 single chromosomal mutations conferring resistance to 16 antibiotics from 8 bacterial species. They reported 8 no-cost mutations, with variable costs of resistance depending on antibiotic class and the species assayed.

Increased resistance to one antibiotic may be accompanied by increased crossresistance to other antibiotics. Cross-resistance is often observed between members of the same class of antibiotic. For example, all quinolones target the essential enzymes DNA gyrase and Topoisomerase IV (Topo IV), whose subunits are encoded by the $g y r A / B$ and $\operatorname{par} C / E$ genes, respectively. Resistance to quinolones can be conferred by point mutations affecting specific portions of GyrA and ParC, known as the quinolone resistancedetermining regions (QRDR) (Horowitz and Wang 1987; Yoshida et al. 1990; Hooper 1999; Ruiz 2003). Changes at amino acid positions 83 and 87 of gyrA lead to a significant loss in quinolone susceptibility. Moreover, known resistance mutations in gyrB mutations affect amino acid positions 426 and 464 , sites that interact with the bound quinolone molecule close to the QRDR of GyrA (Sullivan et al. 1995; Lu et al. 1999; Friedman et al. 2001). Correspondingly, resistance mutations in $g y r A$, confer resistance across quinolones. Moreover, gyrB mutations affecting positions 426 and 464 interact with the bound quinolone molecule close to the QRDR of GyrA, and are known to confer resistance to particular quinolones (Yoshida et al. 1991; Heddle and Maxwell 2002; Weigel et al. 2002). 
$\operatorname{par} C$ mutations are additionally associated with resistance to quinolones (Vila et al. 1996; Heisig 1996). Topo IV acts as a secondary target to quinolones in Gram negatives and $\operatorname{parC}$ mutations are found along with the primary gyrA mutations among clinical isolates (Huseby et al. 2017; Basra et al. 2018). Resistance mutations in the primary target (DNA gyrase) precede those in the secondary target, in a stepwise selection for high-level resistance under prolonged exposure of increasing drug concentrations. In a multistep mutational pattern, particular sets of allele combination of gyrA S83L, D87N, and parC S80I genotype tend to be seen in clinical E. coli isolates at higher frequencies, evolving a high-level clinical resistance (Everett et al. 1996; Bagel et al. 1999; Komp Lindgren et al. 2003; Baudry-Simner et al. 2012; Johnson et al. 2013; Betitra et al. 2014).

Cross-resistance can also occur between drug classes - for example, marR mutations selected by quinolones also confer resistance to phenicols, tetracyclines, and rifampicin (Hooper 1999; Alekshun and Levy 1999a; Alekshun and Levy 1999b). Mutations in $\operatorname{marR}$, which encodes a negative regulator of the $\operatorname{mar} R A B$ operon, render the repressor function inactive, resulting in increased efflux and reduced permeability (Cohen et al. 1993; Hooper 1995; Alekshun and Levy 1997; Alekshun et al. 2001; Randall and Woodward 2002). Clinical strains of $E$. coli resistant to quinolones typically harbor both drug target mutations and efflux pump regulatory mutations (Hooper 1999; Komp Lindgren et al. 2003; Hooper and Jacoby 2015).

By contrast, in collateral sensitivity or negative cross-resistance, acquisition of resistance to one antibiotic may grant sensitivity to other antibiotics. For example, 
resistance to aminoglycosides in $E$. coli can be conferred by electron transport chain mutations that reduce proton-motive force (PMF). This decrease in PMF negatively affects the activity of multi-drug efflux pumps, such as AcrAB-TolC, granting hypersensitivity to many other antibiotics (Imamovic and Sommer 2013; Lázár et al. 2013).

Here, we assess mutation rates, levels of resistance, and pleiotropic effects for chromosomal mutations conferring resistance to different quinolones, in an effort to understand the full set of parameters contributing to the origin, spread, and persistence of quinolone resistance. Quinolones were first used clinically in the 1960's and have undergone multiple rounds of development. The first-generation quinolone, nalidixic acid (NAL), possesses a limited spectrum of activity, but fluorination of the core structure generated the so-called $2^{\text {nd }}$ generation quinolones like ciprofloxacin (CIP). Further overall structural developments resulted in $3^{\text {rd }}$-generation drugs such as levofloxacin (LEVO). We predict that, while broad mechanisms of resistance will be shared between quinolones, resistance mutations will differ in their effects towards different antibiotics owing to differences in antibiotic penetration and/or structural configuration. 


\subsection{Materials and methods}

\subsubsection{Bacterial strains and media}

The E. coli laboratory strain K-12 (MG1655) was used for all experiments. Lysogeny broth (LB) (10 g/l tryptone, $5 \mathrm{~g} / 1$ yeast extract, $10 \mathrm{~g} / 1 \mathrm{NaCl}$ per litre; Bishop) was used for agar and broth cultures.

\subsubsection{Quinolone susceptibility assays}

Minimum inhibitory concentration (MIC) values for the ancestral strain and for antibiotic-resistant mutants were determined for NAL, CIP, and LEVO (Sigma-Aldrich) using a 96 well plate assay in triplicate. Antibiotic concentrations started at $10 \mu \mathrm{g} / \mathrm{ml}$, $1 \mu \mathrm{g} / \mathrm{ml}$, and $8 \mu \mathrm{g} / \mathrm{ml}$ for NAL, CIP and LEVO, respectively, and were diluted in a twofold series and dispensed with $125 \mu \mathrm{l} /$ well of LB into 96 -well plates. The 96 well plates were incubated overnight at $30 \mathrm{C}$, with shaking at $150 \mathrm{rpm}$. The MIC was defined as the lowest concentration of antibiotic for which $90 \%$ growth inhibition was visibly observed after overnight culture. 


\subsubsection{Fluctuation analysis and estimation of mutation rates}

Mutation rates to resistance were determined using fluctuation assays (Luria and Delbrück 1943; Foster 2006). A single colony of E. coli MG1655 was grown overnight in liquid LB at $37 \mathrm{C}$, with shaking at $150 \mathrm{rpm}$. Fresh $200 \mu 1$ cultures were inoculated with $\sim 100$ cells each. Each independent culture was then grown to saturation overnight. The

final number of cells, $\mathrm{N}_{\mathrm{t}}$ was estimated from plate counts on LB without antibiotic.

Selective plates were supplemented with antibiotics (NAL, CIP or LEVO) at a concentration of 1xMIC or 2xMIC. 30 replicate populations were plated for each antibiotic at each concentration. The observed number of mutants, $r$, was then counted for each replicate.

For estimating the number of mutational events $m$, the MSS maximum-likelihood method was used (Ma et al. 1992). This method is based on a recursive algorithm for estimating the Luria-Delbruck distribution for a given number of mutational events (Luria and Delbrück 1943). This method is valid over the entire range of values of $m$ (Sarkar et al. 1992; Hall et al. 2009). The mutation rate per cell per generation, $\mu$, is calculated as $m$ divided by the total number of bacteria plated on selective plates $\left(\mathrm{N}_{\mathrm{t}}\right)$ (Zheng 1999; Rosche and Foster 2000; Foster 2006). The detailed terminology and equations are provided in supplementary Table A2.1 and Box A2.1. 


\subsubsection{PCR amplification and sequencing of candidate genes}

Targeted sequencing of the known resistance loci gyrA and $\operatorname{mar} R$ was carried out in order to identify potential resistance mutations. To ensure independence, a single mutant colony was picked from every plate from the fluctuation assay on which there was growth, and inoculated overnight in LB broth without antibiotic. DNA was then extracted using the EZ-10 spin column bacterial DNA miniprep kit (Bio Basic) and PCR was used to amplify the QRDR of gyrA (Gyrase forward 5'GTAAAACGACGGCCAGTGATGAGCGAC3', Gyrase reverse 5'CGGTACGGTAAGCTTCTTC3') and the entire marR gene (MarR-forward 5'GTAAAACGACGGCCAGTGGTCAATTCA3', MarR reverse 5'TCTGGACATCGTCATACCTC3'). PCR amplicons were sent to Genome Quebec for Sanger sequencing. Mutations in these particular regions were compared with the wild type MG1655 strain of E. coli.

\subsubsection{Whole-genome sequencing}

Whole-genome sequencing (WGS) was carried out to identify potential resistance mutations in clones for which mutations were observed in neither gyrA nor marR. Sequencing libraries were prepared using the Nextera XT kit (Illumina), and sequencing was carried out on the MiSeq platform using paired-end 300bp reads. Raw reads were processed using Trimmomatic-0.35 (Bolger et al. 2014), allowing for a minimum Phredscaled quality score of 20 for leading and trailing bases, truncating reads once average 
quality dropped below 20 in a 4bp sliding window, and dropping all reads of length less than 36. Read quality was assessed using FastQC

\section{(https://www.bioinformatics.babraham.ac.uk/projects/fastqc/).}

Reference-based assembly was carried out using Bowtie-2 (Langmead et al. 2009), with E. coli K-12 (MG1655; NC_00913.2) as the reference genome. SNPs were called using Samtools (Li et al. 2009) and SNP effects were inferred using SNPeff (Cingolani et al. 2012).

\subsubsection{4-hour growth curve analysis}

Fitness in the absence of antibiotic was estimated using 24-hour growth curves for each single colony isolate. Growth curves were obtained in triplicate in 96-well plates, inoculated at a 1:100 dilution from overnight cultures. $\mathrm{OD}_{600}$ was measured on a Bioteck ELx 808 plate reader every 37 minutes for 24 hours, incubating at $37 \mathrm{C}$ with 30 seconds of shaking every 5 minutes. Three growth parameters, lag time, maximum growth rate and optical density at stationary phase after 24 hours, were estimated using the program GrowthRates (Hall et al. 2013). 


\subsection{Results and Discussion}

\subsubsection{Estimation of mutation rates}

We estimated mutation rates to resistance for each of the three quinolones NAL, CIP, and LEVO using fluctuation assays (Luria and Delbrück 1943). The probability of a mutational event per cell per generation was estimated at $1 \mathrm{X}$ and $2 \mathrm{X}$ MIC where MIC values were $10 \mu \mathrm{g} / \mathrm{ml}, 15 \mathrm{ng} / \mathrm{ml}$, and $31 \mathrm{ng} / \mathrm{ml}$ for NAL, CIP and LEVO, respectively. The number of observed mutants (r) on each of 30 selective plates was used to estimate mutation rates to $\mathrm{NAL}^{\mathrm{R}}, \mathrm{CIP}^{\mathrm{R}}$, and $\mathrm{LEVO}^{\mathrm{R}}$ using the MSS maximum likelihood method (Sarkar et al. 1992; Hall et al. 2009). At 1x and 2xMIC, mutation rates differed between antibiotics, with lower rates to $\mathrm{NAL}^{\mathrm{R}}$ than to $\mathrm{CIP}^{\mathrm{R}}$ or $\mathrm{LEVO}^{\mathrm{R}}(\mathrm{CIP}>\mathrm{LEVO}>\mathrm{NAL})$, presumably because fewer resistance mutations are available for NAL that can grant sufficiently high levels of resistance (Supp Table A2.2, Figure 2.2). 


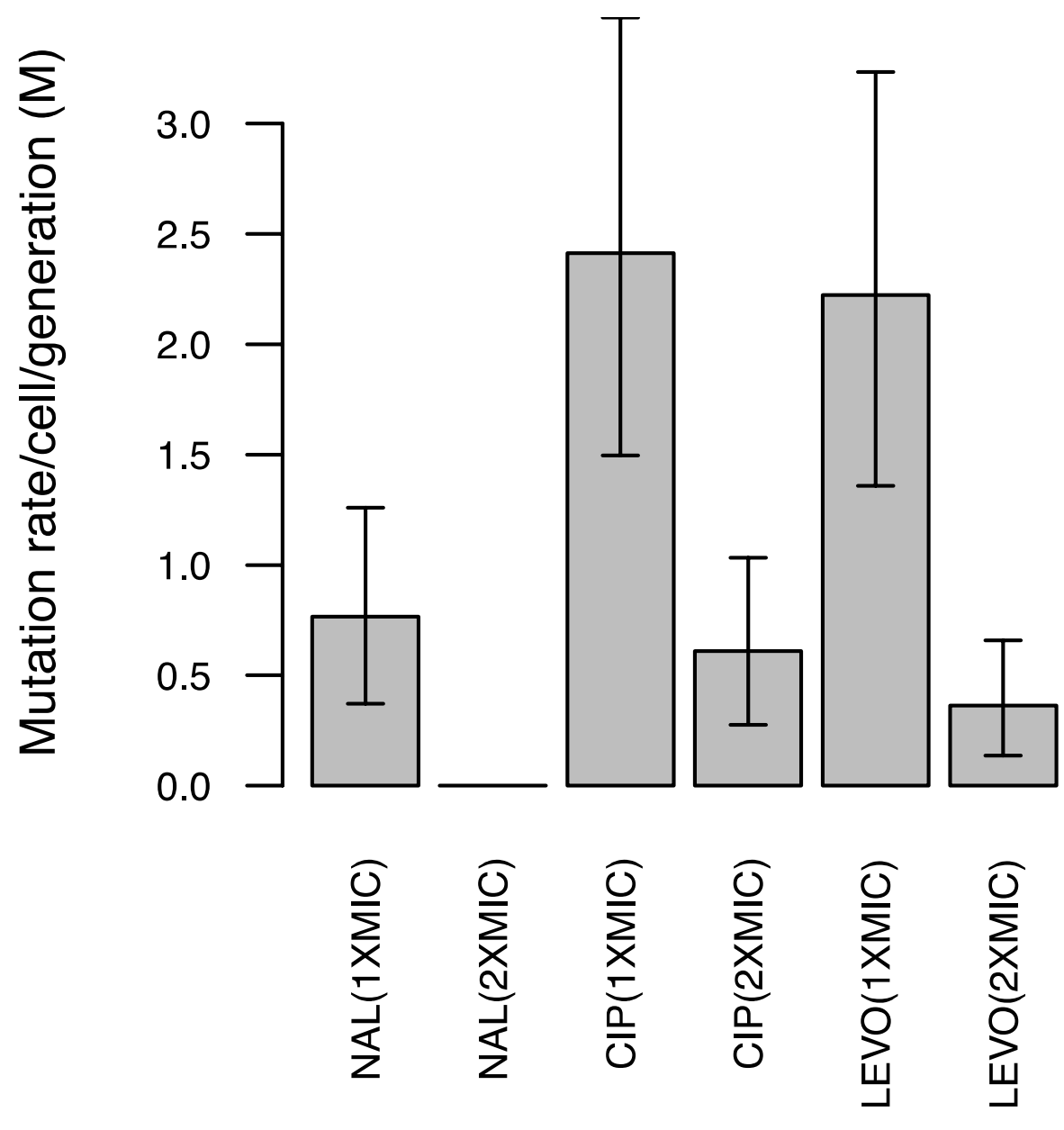

Figure 2.2 Spontaneous mutation rate per $10^{8}$ cells to quinolone resistance among $E$. coli K-12 (MG1655). The mutation rate was estimated from 30 independent cultures at $1 \mathrm{X}$ and $2 \mathrm{X}$ MIC concentration. Error bars represent $95 \%$ confidence intervals. Note that no colonies were obtained at $2 \mathrm{xMIC}$ for NAL. 


\subsubsection{Identification of resistance mutations}

We obtained a total of 56 spontaneous quinolone resistant mutants from the fluctuation assays (one from each plate), and identified putative resistance mutations in 50 of these (Table 2.1, Supp Table A2.3). Targeted Sanger sequencing identified 36 gyrA mutants, 9 marR mutants, and 1 double gyrA/marR mutant. We performed WGS on remaining of the 10 resistant mutants and identified an additional 4 gyr $B$ mutants. No mutations were identified in rest of the 6 resistant mutants, as WGS might not be able to detect big deletions or duplications. We note that $\operatorname{gyr} A / g y r B$ or $\operatorname{marR} /$ gyrB double mutants will be undetected by our approach. However, given that only one gyrA/marR double mutant was detected, the frequency of double mutants is probably low. In gyr $A$, mutations were observed at nucleotides encoding amino acid positions 67, 81, 83, and 87 (Figure 2.3A), consistent with previous findings that the GyrA QRDR spans amino acid 67 to 107 (Horowitz and Wang 1987; Yoshida et al. 1990; Heisig and Tschorny 1994; Hopkins et al. 2005). The most common alterations in the gyrA QRDR region were $\mathrm{S} 83 \mathrm{~L}(\mathrm{n}=18)$ and $\mathrm{D} 87 \mathrm{G} / \mathrm{N} / \mathrm{Y}(\mathrm{n}=11)$. These mutations were found in mutants isolated against all three drugs, presumably because these positions are located within the positively charged region close to the DNA-enzyme binding site (Hooper 1999). Amino acids 83 and 87 are located near the active site of DNA gyrase, along with the tyrosine-122 residue that interacts with the broken DNA strand following cleavage (Oram and Fisher 1991; Maxwell 1992; Everett et al. 1996; Heisig 1996; Hopkins et al. 2005; Komp-Lindgren et al. 2005; Marcusson et al. 2009; Moon et al. 2010). The $\alpha$ helix-4 region is particularly essential to quinolone binding and the substitution to leucine at position 83 makes the 
vicinity of $\alpha$ helix- 4 of the gyrase less electron rich, crippling gyrase-quinolone binding (Hallett and Maxwell 1991; Maxwell 1992; Lu et al. 1999). Mutations at position 87 and 81 also perturb the alignment of the $\alpha$ helix-4 structure.

marR mutations were obtained only in $\mathrm{LEVO}^{\mathrm{R}}$ isolates. These point, frameshift, and missense mutations were dispersed throughout the gene, as expected given that the Mar phenotype can arise from any loss-of-function mutation (Figure 2.3C). MarR consists of two domains, one N-C terminal domain and a helix-turn-helix (HTH) DNA binding domain. The closely packed hydrophobic core and intermolecular hydrogen bonds stabilize the $\mathrm{N}$-terminus (residues 10-21) of one subunit and the C-terminus (residues 123-144) of the second subunit, holding the dimer together. Some of the mutations reported in this study belong to the oligomerization dimer domain of MarR, such as those at positions 10,27, and 33 of one terminal subunit, and positions 123 and 126 of the other equivalent subunit. The rest of the MarR protein is linked via antiparallel helices emerging out from each of the subunits, encompassing the DNA binding domain (residues 55-100) (Alekshun et al. 2000; Alekshun et al. 2001). Mutations at positions 64 and 69 fall in the HTH DNA binding motif. Other mutations reported here at position 42 and 102 residue at $\alpha$ and $\beta$ sheets of MarR, which are essential for the interaction between the two antiparallel strands of HTH DNA binding domain (Alekshun et al. 2001). We note that previous work has identified marR mutants (R77H, R94C, and $\Delta 72-$ 82) during laboratory adaptation of E. coli to CIP (Wong and Seguin 2015). A possible explanation for our lack of marR mutants on CIP may be that marR mutants were growing slower on NAL and CIP than were gyrA or gyrB mutants. 
WGS revealed gyrB mutations in four isolates for which no gyrA or marR mutations were detected by Sanger sequencing (Figure 2.3B). H281L, S464F, and L509Q mutations were found in strains isolated on CIP, and a D426N mutation was found in a strain isolated on NAL. The GyrB enzyme consists of two domains, an N-terminal domain (amino acid 2-393) that incorporates the ATPase catalytic site, and a C-terminal domain (amino acid 394-804) that interacts with GyrA (Reece and Maxwell 1991; Brino et al. 2000). D426N and S464F have been previously reported to confer quinolone resistance (Nakamura et al. 1989; Yoshida et al. 1991; Vila et al. 1996; Hopkins et al. 2005; Komp-Lindgren et al. 2005). Both of these mutations are part of the QRDR of GyrB and cause conformational changes in the structure of the gyrase subunits (Weigel et al. 2002; Hopkins et al. 2005). The gyrB D426N mutation has been reported before along with a mutation at position L447, both of which provide a neutral vicinity, owing to their respective opposite charges. Both of these residues are suggested to be part of a quinolone-binding pocket (Yoshida et al. 1991; Gensberg et al. 1995; Hooper 1999; Heddle and Maxwell 2002).

Interestingly, the H281L and L509G mutations have not been previously reported to confer quinolone resistance. These two novel mutations are located outside the GyrB QRDR. Position 281 is located in the transducer region of GyrB, which forms a cavity just large enough to facilitate the transfer of the trapped double-stranded DNA through the DNA gate in the presence of ATP (Kampranis et al. 1999; Champoux 2001; Sissi and Palumbo 2010; Stanger et al. 2014). Position 509 is within the TOPRIM domain of GyrB, part of the catalytic DNA cleavage-rejoining complex along with the GyrA winged helix domain (Sissi and Palumbo 2010; Gubaev and Klostermeier 2014). 
Table 2.1 MIC fold-increase and mutations in $\operatorname{gyr} A, \operatorname{gyr} B$, and $\operatorname{mar} R$ among $\mathrm{NAL}^{\mathrm{R}}, \mathrm{CIP}^{\mathrm{R}}$, and $\mathrm{LEVO}^{\mathrm{R}}$ mutants of E. coli $\mathrm{K}-12$ (MG1655).

\begin{tabular}{|c|c|c|c|c|c|c|c|c|c|}
\hline \multirow[t]{2}{*}{ Antibiotic } & \multirow[t]{2}{*}{$\begin{array}{l}\text { MIC } \\
\text { WT } \\
\text { (ug/ml) }\end{array}$} & \multicolumn{3}{|c|}{$\begin{array}{l}\text { MIC fold-change } \\
\text { of mutants }\end{array}$} & \multicolumn{3}{|c|}{ Resistance mutation } & \multirow{2}{*}{$\begin{array}{l}\text { Total no. } \\
\text { of } \\
\text { NAL }^{\mathrm{R}}, \\
\text { CIP }^{\mathrm{R}}, \\
\text { and } \\
\text { LEVO }^{\mathrm{R}} \\
\text { mutants }\end{array}$} & \multirow{2}{*}{$\begin{array}{l}\text { No. of } \\
\text { gyr } A \text {, } \\
\text { gyrB, and } \\
\text { marR } \\
\text { mutants }\end{array}$} \\
\hline & & NAL & CIP & LEVO & gyrA & $g y r B$ & marR & & \\
\hline \multirow[t]{5}{*}{ Nalidixic acid } & 10 & & & & & & & 11 & \\
\hline & & 64 & & & A67S & & & & 2 \\
\hline & & 256 & & & S83L & & & & 6 \\
\hline & & 128 & & & D87G & & & & 2 \\
\hline & & 64 & & & & $\mathrm{D} 426 \mathrm{~N}$ & & & 1 \\
\hline \multirow[t]{8}{*}{ Ciprofloxacin } & 0.015 & & & & & & & 24 & \\
\hline & & & 64 & & G81C/D & & & & 3 \\
\hline & & & 128 & & S83L & & & & 4 \\
\hline & & & 32 & & S83W & & & & 2 \\
\hline & & & 64 & & $\mathrm{D} 87 \mathrm{Y} / \mathrm{G} / \mathrm{N}$ & & & & 7 \\
\hline & & & 32 & & & $\mathrm{H} 281 \mathrm{~L}$ & & & 1 \\
\hline & & & 32 & & & S464Y & & & 1 \\
\hline & & & 16 & & & L509G & & & 1 \\
\hline \multirow[t]{14}{*}{ Levofloxacin } & 0.031 & & & & & & & 21 & \\
\hline & & & & 16 & G81C & & & & 1 \\
\hline & & & & 16 & S83L & & & & 8 \\
\hline & & & & 16 & D87G & & & & 1 \\
\hline & & & & 16 & D87G & & $\mathrm{R} 27 \mathrm{P}$ & & 1 \\
\hline & & & & 16 & & & E10stop & & 1 \\
\hline & & & & 16 & & & L33R & & 1 \\
\hline & & & & 8 & & & Q42E & & 1 \\
\hline & & & & 8 & & & L64fs & & 1 \\
\hline & & & & 8 & & & G69E & & 1 \\
\hline & & & & 8 & & & $\mathrm{~T} 102 \mathrm{~S}$ & & 1 \\
\hline & & & & 8 & & & Q117stop & & 1 \\
\hline & & & & 4 & & & L123S & & 1 \\
\hline & & & & 8 & & & N126fs & & 1 \\
\hline
\end{tabular}




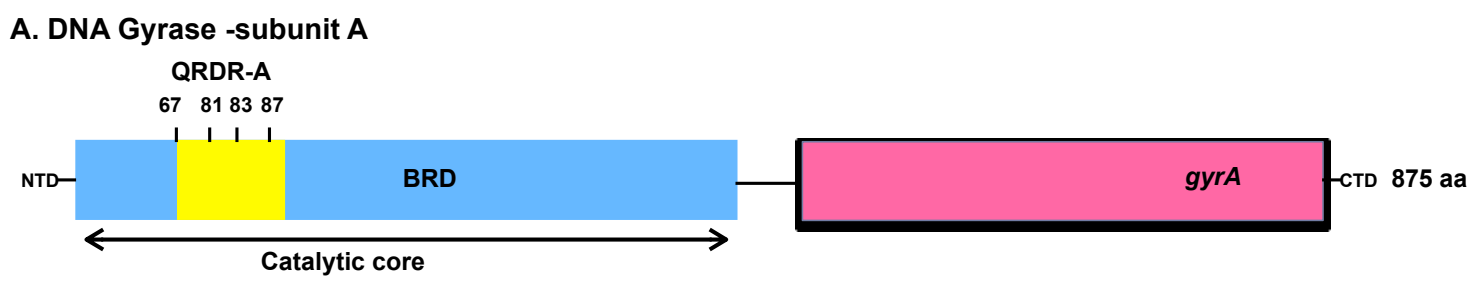

B. DNA Gyrase -subunit B

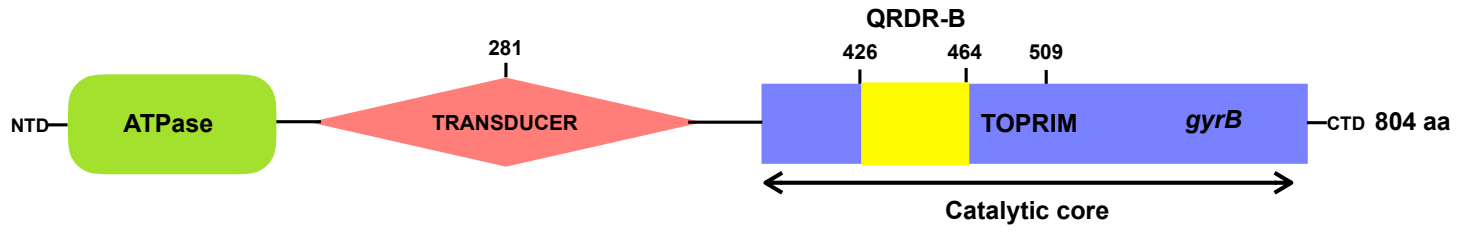

C. MarR

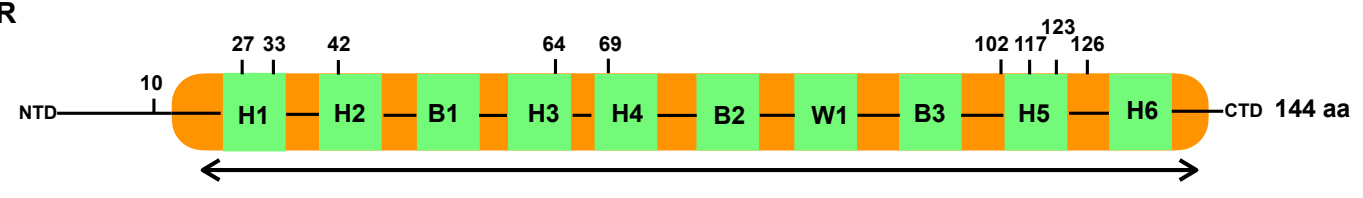

HELIX-TURN-HELIX

Figure 2.3 The domain structures of gyrase A, B, and MarR. Mutations obtained in this study are indicated in bold above. Panel A: Arrangement of GyrA. This subunit of DNA gyrase consists of the breakage-reunion (BRD) domain, and the quinolone resistance determining region (QRDR-A) site. Panel B: Arrangement of GyrB, with the ATPase, Transducer (221-392), and Toprim (418-533) regions. The QRDR-B is shown within the Toprim domain of GyrB (Stanger et al. 2014). Panel C. MarR domain structure, comprising four helices $(\mathrm{H})$ and three $\beta$-sheets (B). H3 and $\mathrm{H} 4$ (57-80) are the recognition and DNA binding motifs containing $\mathrm{H}-\mathrm{T}-\mathrm{H}$ motifs and the $\beta$-sheet winged structure. H1, H5, and H6 are associated with dimerization (Sulavik et al. 1995; Alekshun et al. 2001; Finn et al. 2016; Uniport Consortium 2018). 


\subsubsection{Direct responses to selection}

An increase in fitness in the selective environment is referred to as the direct response to selection; here, the direct response to selection is measured by an increase in MIC towards the drug on which a mutant was selected. We found substantial variation between drugs in the magnitude of the direct response. Mutants isolated on NAL showed a stronger direct response to selection than did mutants isolated on CIP or LEVO, with a mean increase of 256-fold MIC towards NAL. Mutants isolated on CIP and LEVO showed mean increases of 64-fold and 16-fold towards CIP and LEVO, respectively (Figure 2.4).

Among resistance mutations, gyrA mutations consistently showed higher levels of resistance than $g y r B$ or $\operatorname{mar} R$, regardless of the antibiotic they were isolated on, which impacts the variation in MIC values significantly (Table 2.2). Furthermore, within each gene, the level of resistance varied by mutation. In the case of gyr $A$, the S83L mutation conferred higher resistance among all the isolates compared to other mutational sites of gyrA $(87,81$ or 67$)$. This suggests that the widespread occurrence of the S83L mutation amongst clinical isolates is due to the high level of resistance (Supp Figure A2.1). 

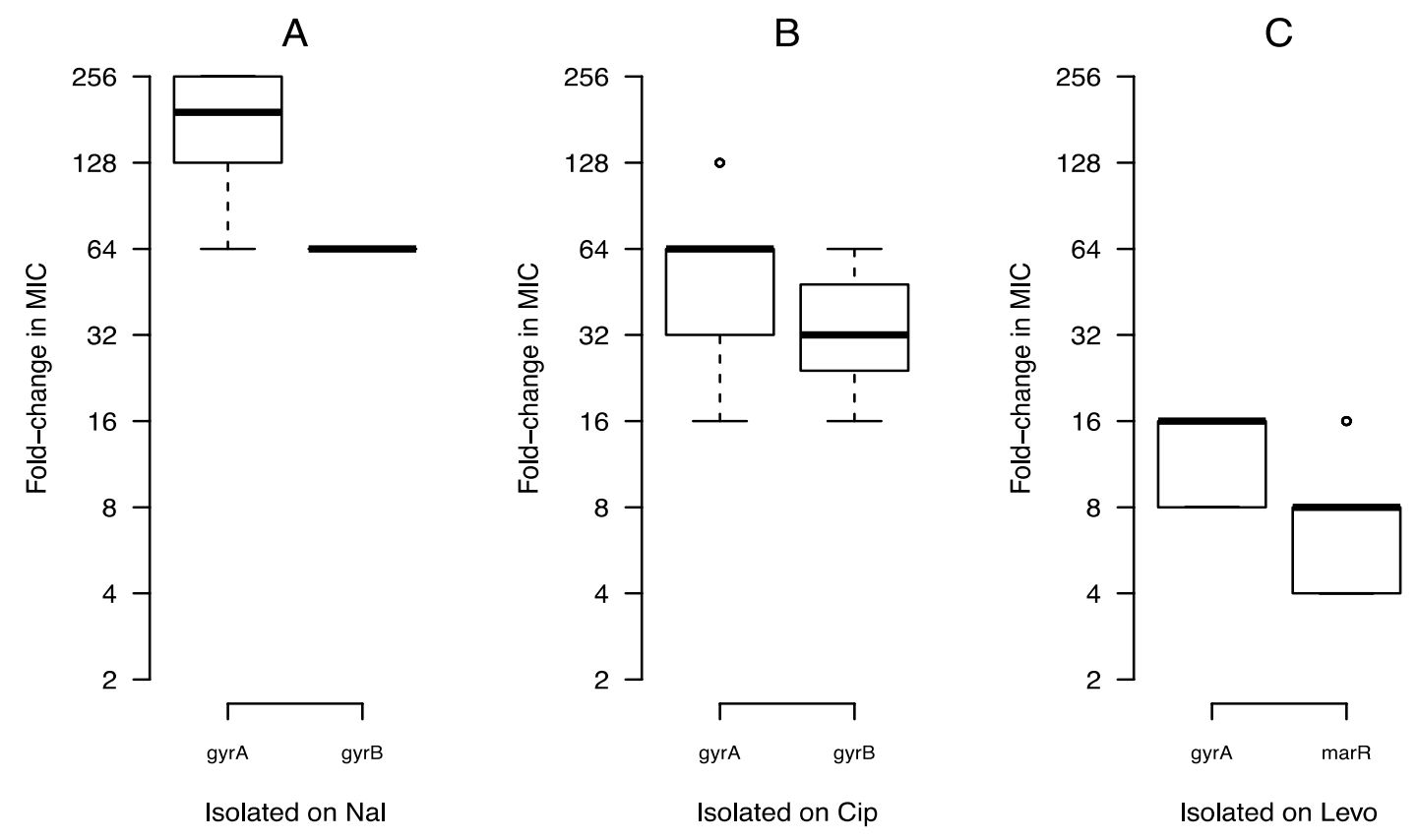

Figure 2.4 Direct responses to selection. Changes in MIC for resistant mutants towards the drug on which they were selected: NAL (A), CIP (B), and LEVO (C). The boxplot presents the median, first, and third quartiles, with whiskers showing either the maximum (minimum) value or 1.5 times the interquartile range of the data, whichever is smaller (larger). The points (open circles) indicate the outliers. 
Table 2.2 Two-way analysis of variance (ANOVA) for the effects of antibiotic and gene on levels of resistance.

\begin{tabular}{lll}
\hline Factor & F-value & $\boldsymbol{P}$-value \\
\hline Gene & 10.00 & $0.00025^{*}$ \\
Antibiotic & 39.30 & $1.34 \mathrm{e}-10^{*}$ \\
Gene*Antibiotic & 2.83 & 0.09 \\
\hline
\end{tabular}

\subsubsection{Cross resistance between quinolones}

Cross-resistance between quinolones was widespread: all of the resistant mutants isolated on one quinolone displayed increased resistance, in varying degrees, to the other two quinolones. Nonetheless, different quinolones were not equally affected by the resistance mutations (Figure 2.5). Overall, mutants were more resistant towards NAL than they were towards CIP or LEVO. Among CIP and LEVO, mutations showed smaller gains in resistance on LEVO. Nevertheless, significant correlations between levels of resistance for CIP and LEVO (Pearson's $r=0.64, t=6.2, P=8.433 \mathrm{e}-08)$, NAL and CIP $(r=0.47, t$ $=3.93, \mathrm{P}=0.0002)$, and NAL and LEVO $(\mathrm{r}=0.38, \mathrm{t}=3.07, \mathrm{P}=0.0032)$ suggest a closer relationship between the newer quinolones. This variation in resistance among quinolones can be explained by the intrinsic structural drug differences between older and newer quinolone classes. NAL is devoid of any cyclic derivatives whereas CIP and LEVO have substituents at positions C-6, C-7, and C-8 that offer greater 
spectrum/potency of activity. Thus, the modified quinolone substituents likely reduce resistance levels by increasing the affinity for GyrA, and by stabilizing the quinoloneDNA complex (Domagala 1994; Tillotson 1996; Zhao et al. 1998; Dong et al. 1999; Fukuda and Hiramatsu 1999; Jorgensen et al. 1999; Lu et al. 1999; Pestova et al. 2000; Barnard and Maxwell 2001; Peterson 2001; Sanders 2001; Zhao and Drlica 2001; Michot et al. 2005; Becnel et al. 2009; Morgan-Linnell et al. 2009; Azéma et al. 2011).

These trends are also evident for specific loci. gyr $A$ mutants showed the highest gain in resistance (average 128XMIC) on NAL in comparison with CIP (32XMIC) or LEVO (16XMIC). gyrB mutants also displayed a higher increase in resistance to NAL (64XMIC), but the same increase on CIP and LEVO (16XMIC). The novel H281L and L509G mutations gained similar increases with CIP and LEVO, at 32XMIC and 16XMIC respectively. On NAL, H281L gained 64XMIC whereas L509G gained similar increase in resistance as with CIP or LEVO, i.e. 16XMIC. Meanwhile, marR mutants did not show as high increases in MICs, with increases of 32X, 16X, and 8XMIC on NAL, CIP and LEVO. 

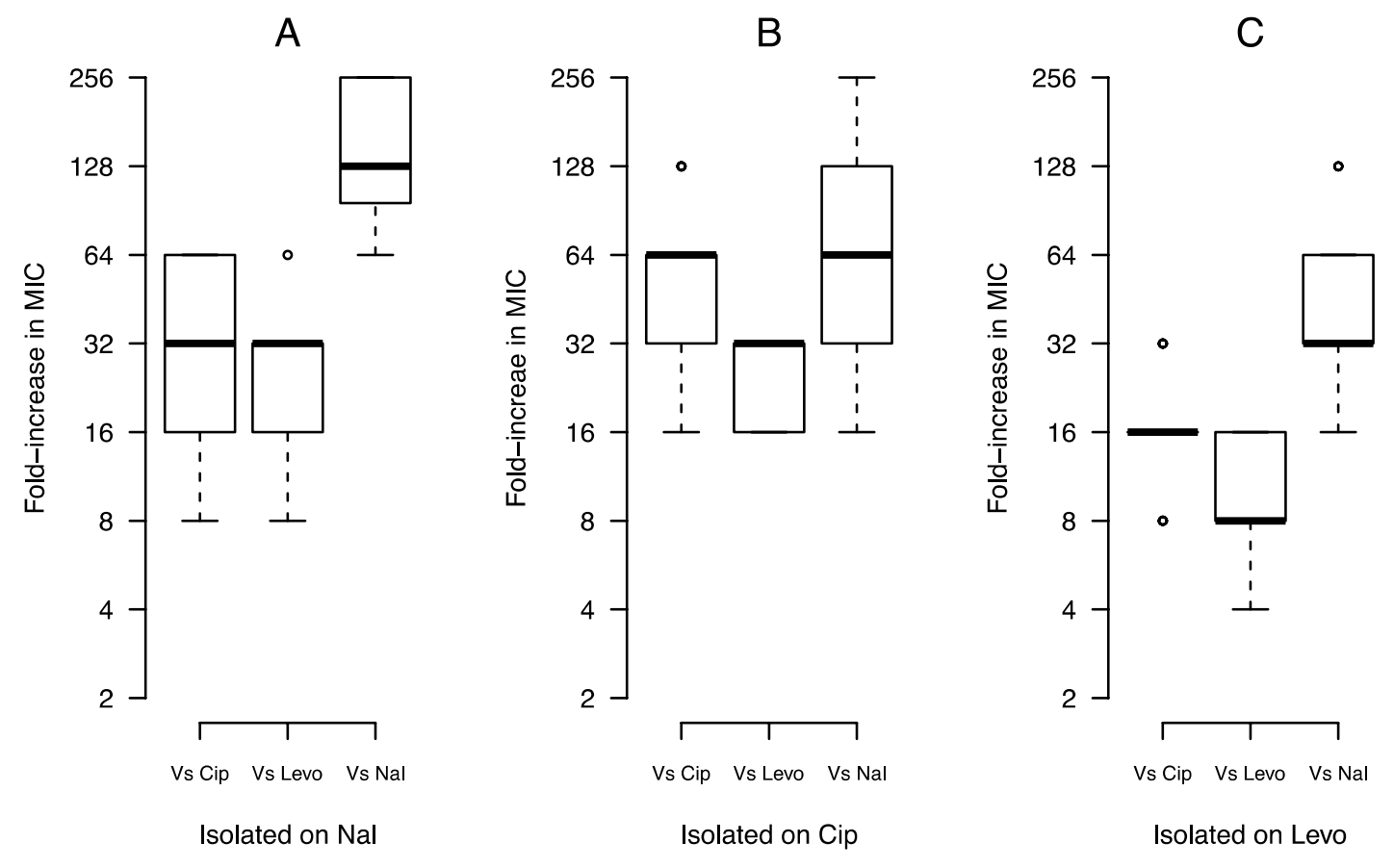

Figure 2.5 Cross-resistance between resistant mutants. Fold-increase in MIC of resistant mutants isolated on NAL (A), CIP (B), and LEVO (C) against all three antibiotics. The boxplot presents the median, first, and third quartiles, with whiskers showing either the maximum (minimum) value or 1.5 times the interquartile range of the data, whichever is smaller (larger). The points (open circles) indicate the outliers. 


\subsubsection{Cost of resistance}

The persistence of resistance in the absence of antibiotic is determined in part by the fitness costs associated with resistance mutations (Björkman et al. 2000; Gagneux et al. 2006; Andersson and Hughes 2010). No-cost mutations may contribute to the persistence of resistance mutations in the absence of antibiotic. We measured three fitness components in the absence of antibiotic for our set of quinolone resistant mutants: maximum growth rate (Vmax), density at stationary phase (Max OD), and length of lag phase. Resistant mutants were consistently found to be costly, exhibiting significant differences in Vmax, Max OD, and lag time compared to their drug-susceptible ancestor MG1655 (Figure 2.6, Table 2.3) (Supp Figure A2.2 A, B, C). Thus, overall, we observe significant costs of resistance for quinolone resistance mutations, consistent with previous studies (Bagel et al. 1999; Barnard and Maxwell 2001; Kugelberg et al. 2005; MacLean and Vogwill 2014; Melnyk et al. 2015). 

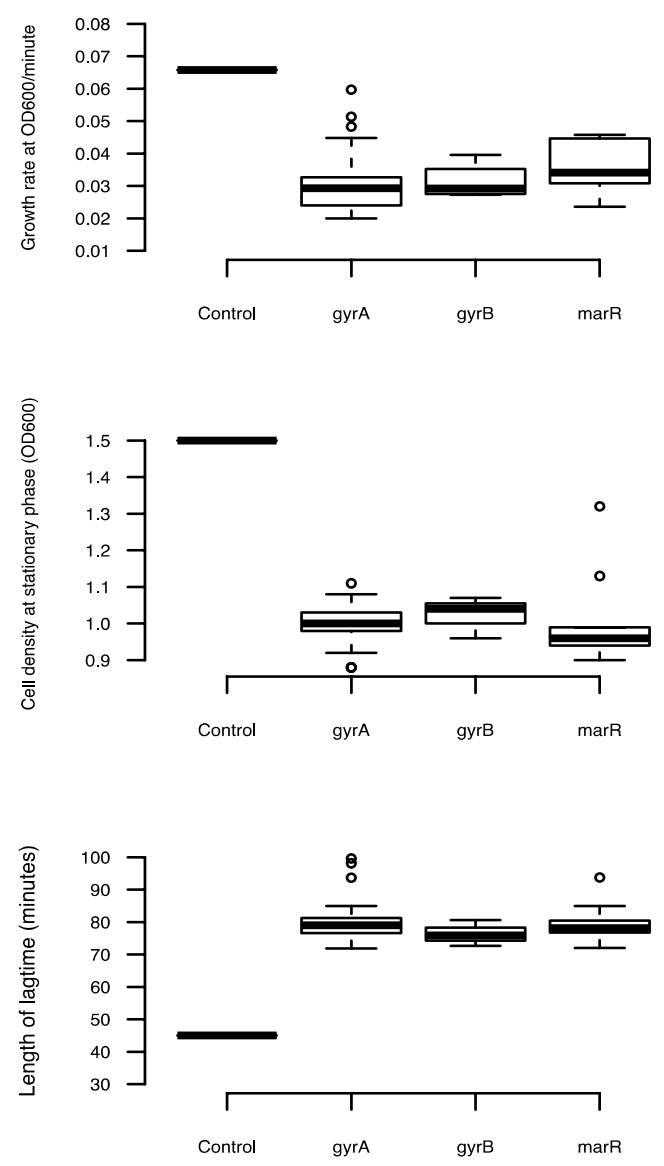

Figure 2.6 Cost of resistance of quinolone resistant mutants. The fitness components measured are growth rate, cell density, and lag time between $\operatorname{gyr} A, \operatorname{gyr} B$, and $\operatorname{mar} R$ resistance mutations. All the fitness components are compared to control E. coli $\mathrm{K}-12$ (MG1655). The boxplot presents the median, first, and third quartiles, with whiskers showing either the maximum (minimum) value or 1.5 times the interquartile range of the data, whichever is smaller (larger). The points (open circles) above or below the whiskers indicate the outliers. 
Table 2.3 Effects of resistance mutations on growth parameters.

\begin{tabular}{|c|c|c|c|c|c|c|}
\hline \multirow[t]{3}{*}{ Factor } & \multicolumn{6}{|c|}{ Post hoc (Tukey HSD) comparisons with $E$. coli K-12 MG1655 } \\
\hline & \multicolumn{2}{|c|}{$\begin{array}{l}\text { Growth rate } \\
\text { (OD600/minute) }\end{array}$} & \multicolumn{2}{|c|}{$\begin{array}{l}\text { Length of } \\
\text { lag time (minutes) }\end{array}$} & \multicolumn{2}{|c|}{$\begin{array}{c}\text { Cell density at } \\
\text { stationary phase } \\
\text { (OD600) }\end{array}$} \\
\hline & Mean & $P$-value & Mean & $P$-value & Mean & $P$-value \\
\hline $\begin{array}{l}\text { gyrA- } \\
\text { MG1655 }\end{array}$ & -0.032 & 0.0000011 & 33.4 & $<2.0 \mathrm{e}-16$ & -0.55 & $<2.0 \mathrm{e}-16$ \\
\hline $\begin{array}{l}\text { gyrB- } \\
\text { MG1655 }\end{array}$ & -0.033 & 0.0000191 & 28.9 & $<2.0 \mathrm{e}-16$ & -0.52 & $<2.0 \mathrm{e}-16$ \\
\hline $\begin{array}{l}\operatorname{marR}- \\
\text { MG1655 }\end{array}$ & -0.029 & 0.0000173 & 32.3 & $<2.0 \mathrm{e}-16$ & -0.53 & $<2.0 \mathrm{e}-16$ \\
\hline
\end{tabular}

Some studies have reported that mutations granting higher levels of resistance impose higher costs (Melnyk et al. 2015). However, we fail to find a significant relationship between MIC and any fitness component. No correlations were found between MIC and growth rate $(\mathrm{P}=0.42$, tau $=0.08)$, length of lag phase $(\mathrm{P}=0.82$, tau $=$ $0.02)$ or cell density $(\mathrm{P}=0.12$, tau $=0.15)$ (Figure 2.7$)$. We note that gyrA mutations confer no greater costs than other resistance mutations ( $g y r B$, marR). Moreover, amongst the handful of mutations in gyrA (S83, D87, G81) that can confer high level resistance (Heisig and Tschorny 1994; Heisig 1996), a few prominent alleles of gyrA tend to be found in E. coli clinical isolates (Everett et al. 1996; Komp-Lindgren 2003; Betitra et al. 2014; Basra et al. 2018). That these mutations confer high levels of resistance, but are no more costly than other gyrA mutations, could help to explain the prevalence of specific gyrA mutations amongst clinical isolates. 

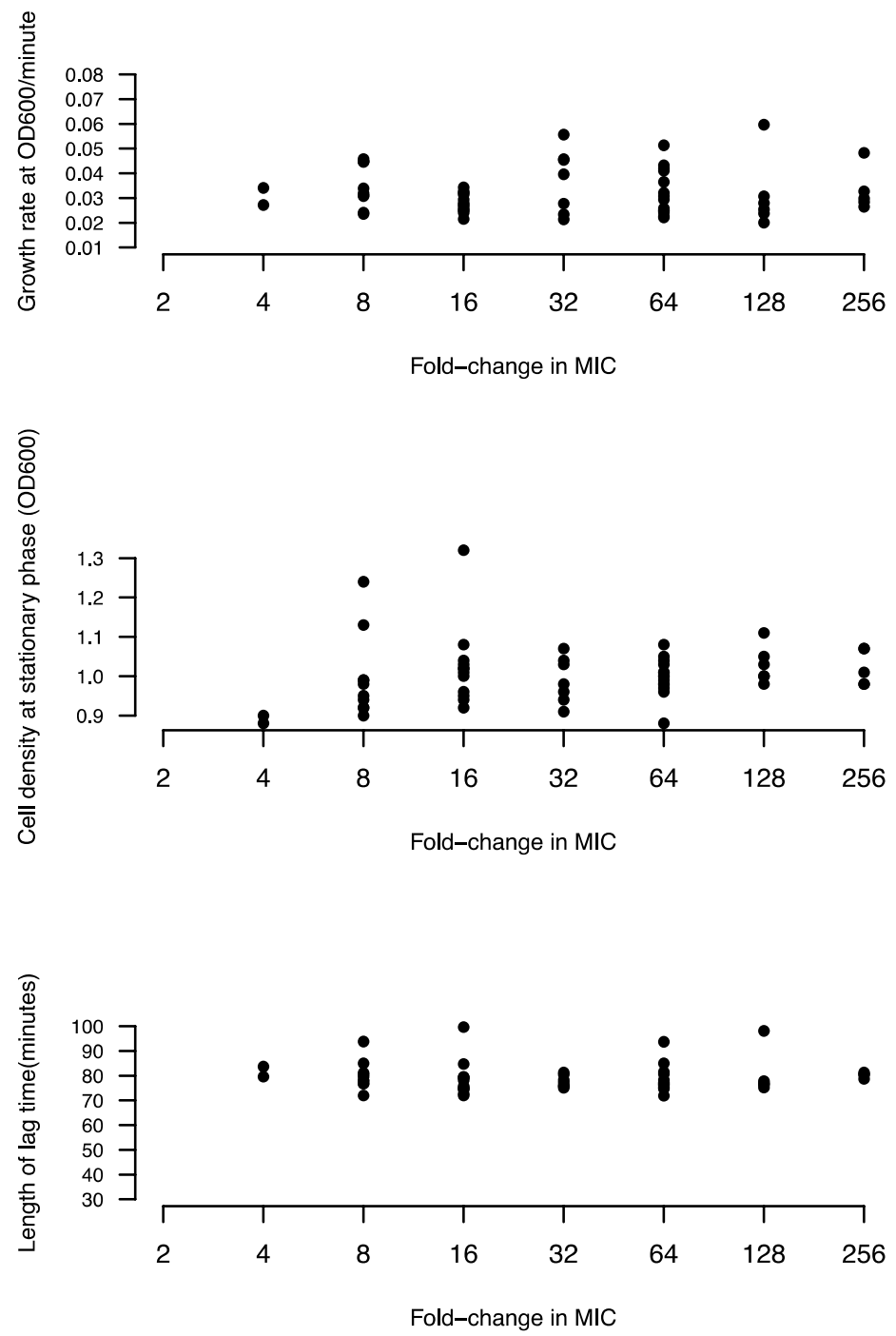

Figure 2.7 No correlation between level of resistance (fold-change in MIC) and growth rate, cell density or length of lag phase for all mutants. 


\subsection{Conclusions and Perspectives}

Quinolones target DNA gyrase and topoisomerase IV. Resistance against them is achieved by target alteration and/or through efflux and permeability associated mutations. We find that 50/56 spontaneous mutants obtained through fluctuation assays were resistant through mutations in the known resistance conferring genes - $\operatorname{gyr} A(36), \operatorname{gyr} B(4), \operatorname{mar} R(9)$, and $\operatorname{gyr} A / \operatorname{mar} R(1)$. This finding suggests that there are few other quinolone resistance mutations available in E. coli $\mathrm{K}-12$; this is somewhat surprising, given that selection experiments in $P$. aeruginosa have identified novel resistance mutations (Wong et al. 2012). We find significant costs of resistance, and differences in the mutational supply rate among resistant isolates. Notably, we find gyrA mutations conferred higher resistance, without greater fitness cost, than other mutations. This finding may explain the prevalence of gyr $A$ mutations in clinical samples. We also find variation in cross-resistance amongst quinolone resistant isolates, implying that different resistance mutations respond differently to quinolone variants. Thus, antibiotic variants may have different implications for the evolution of resistance. Optimally, we should choose an antibiotic for which resistance is costly, and where single mutations have relatively small effect, as was the case here for levofloxacin. 


\section{Chapter 3}

\section{An sRNA screen for reversal of quinolone resistance in Escherichia coli}

\subsection{Abstract}

In light of the rising prevalence of antimicrobial resistance (AMR) and the slow pace of new antimicrobial development, there has been increasing interest in the development of adjuvants that improve or restore the effectiveness of existing drugs. Here, we use a novel small RNA (sRNA) screening approach to identify genes whose knockdown increases ciprofloxacin (CIP) sensitivity in a resistant strain of Escherichia coli. 5000 constructs were initially screened on a gyrA S83L background, ultimately leading to 30 validated genes whose disruption reduces CIP resistance. This set includes genes involved in DNA replication, repair, recombination, efflux, and other regulatory systems. Our findings increase understanding of the functional interactions of DNA gyrase, and may aid in the development of new therapeutic approaches for combating AMR. 


\subsection{Introduction}

The rapid evolution of antimicrobial resistance (AMR) among bacterial pathogens and the arrested development of new antibiotics have driven the search for novel approaches to counteract the resistance crisis (Levy and Marshall 2004; Spellberg 2004; Payne 2007; Davies and Davies 2010). Recently, re-sensitization of resistant strains to existing antibiotics has emerged as a promising strategy (Wright 2000; Bassetti et al. 2008). Compounds that reduce bacterial resistance, and that thereby restore the effectiveness of existing drugs, are a promising variety of antibiotic adjuvant (Wright 2016; GonzálezBello 2017). Certain ß-lactamase inhibitors, for example, restore susceptibility to cephalosporins by inhibiting degradative enzymes (extended-spectrum B-lactamases, ESBLs) that are often responsible for resistance (Drawz and Bonomo 2010; King et al. 2014).

Here, we use a bacterial small RNA (sRNA) screen to identify genes whose knockdown re-sensitizes DNA gyrase-mediated resistance to the fluoroquinolone antibiotic ciprofloxacin (CIP). CIP is a synthetic antibiotic used globally for the treatment of many bacterial infections (Hooper 1999; Anderson 2004; Bolon 2011); high-level resistance is typically conferred by mutations in the gyrA gene, which encodes one subunit of DNA gyrase, the primary target of quinolones (Drlica and Zhao 1997; Walsh 2000). The S83L substitution in the GyrA subunit confers a high-level of CIP resistance in E. coli (Bagel 1999; Bhatnagar and Wong submitted). We reasoned that CIP 
susceptibility might be restored in gyr $A$ mutants by disrupting genes involved in the function of DNA gyrase, or by altering cell permeability to CIP.

Bacterial sRNAs are widespread, non-coding RNA molecules. They are typically 50-300 nucleotides in size, trans-encoded, with distinct stem loops as secondary structures (Argaman et al. 2001; Gottesman 2004; Vogel and Sharma 2005, Sharma and Vogel 2009; Yoo et al. 2013). They play a prominent role in bacterial physiology by controlling gene expression post-transcriptionally. Each sRNA consists of two important regions. One is the recognition region that regulates sRNA-mRNA base-pairing through antisense short complementary base-pairing with the 5' untranslated region (UTR) or translation initiation region (TIR), and the other is the scaffold (Hfq) region that stabilizes sRNA-mRNA base-pairing (Møller et al. 2002; Zhang et al. 2002; Storz et al. 2004; Jousselin et al. 2009; Vogel and Luisi 2011; Holmquist and Vogel 2013; VazquezAnderson and Contreras 2013; Lee and Moon 2018; Lee et al. 2019). Binding of sRNA to mRNA targets can reduce gene expression by inhibiting translation or promoting mRNA degradation.

In eukaryotes, RNA interference (RNAi) is used extensively for studies of gene function. RNAi mediated gene silencing through short interfering (siRNA) and short hairpin (shRNA) RNAs has become a mainstay in cancer research and is a recognized basis of target validation and drug development (Silva et al. 2005, 2008; Schlabach et al. 2008; Scholl et al. 2009). In prokaryotes, comparable use of sRNA as a genetic tool is promising, but still needs to be explored further (Nakashima et al. 2006; Man et al. 2011; Meng et al. 2012; Sharma et al. 2011, 2013). Sharma et al. $(2011,2013)$ have exploited 
the modular nature of natural bacterial sRNAs and with a fused fluorescent protein (GFP) as reporter, successfully isolated and characterized three artificial sRNAs. These artificial sRNAs post-transcriptionally repressed the endogenous gene expression of ompF (encoding an outer-membrane porin), fliC (a motility gene), and $r e c A$ (involved in DNA repair) in E. coli. recA downregulation subsequently led to increased susceptibility to CIP. Similarly, Man et al. (2011) also designed artificial sRNAs for specific gene silencing in bacteria. Their synthesized sRNAs downregulated the expression of exogenous (EGFP) and endogenous (uidA) genes in E. coli.

In the context of AMR, Lee et al. (2011) demonstrated the potential of sRNA screens for the reversal of antibiotic resistance. They conducted a genetic screen using antisense RNA interference conditional expression against 245 essential genes for reversal of AMR in methicillin-resistant S. aureus (MRSA) to restore B-lactam activity. The library was expressed on wild-type as well as resistant MRSA strains (COL and USA300) under partially inducing conditions. They found 45 genes whose knockdown reduced resistance to at least one of seventeen clinically relevant antibiotics, including those involved in cell division ( $f$ ts $Z$ and $f t s A$ ), protein secretion (spsB), teichoic acid biosynthesis $(\operatorname{tar} L)$, and signal transduction. Notably, since these are essential genes, they would be missed in any knockout-driven screening approach, highlighting a key benefit of sRNA screens.

In this study, we randomized the antisense sequences of three naturally occurring sRNAs to generate an sRNA expression library with the potential to target diverse mRNA transcripts. We identified a number of sRNA sequences that reduce quinolone 
resistance on a gyrA S83L background. Further bioinformatic and functional analyses confirmed several genes whose down-regulation reduces resistance levels, and that may thus be promising adjuvant targets. 


\subsection{Materials and Methods}

\subsubsection{Bacterial strains, media, and plasmid construction}

One shot Top10 E. coli (Invitrogen, F- mcrA $\Delta$ (mrr-hsdRMS-mcrBC), j80lacZ $\Delta$ M15, $\Delta$ lacX74 recA1 araD139 $\Delta$ (ara leu)7697, galU, galK, rpsL (StrR), endA1, nupG)

chemically competent cells were used for the development of randomization methods and for vector maintenance. For the sRNA screen, a quinolone resistant derivative of $E$. coli K-12 (MG1655) was generated by gene gorging (Herring 2003). Briefly, a fragment of gyrA encoding the S83L substitution (via a TCG->TTG mutation) and an I-SceI restriction site was generated by megaprimer PCR (Herring 2003) and cloned into the PCR2.1 vector using TOPO cloning (Invitrogen). This donor plasmid was co-transformed into $E$. coli $\mathrm{K}-12$ (MG1655) along with the mutagenesis plasmid pACBSR. I-SceI endonuclease and $\lambda$-red functions encoded on pACBSR were then induced with arabinose. Potential mutants were plated on $\mathrm{LB}$, and replica plated to $\mathrm{LB}+50 \mu \mathrm{g} / \mathrm{ml}$ kanamycin, $\mathrm{LB}+25 \mu \mathrm{g} / \mathrm{ml}$ chloramphenicol, or $\mathrm{LB}+25 \mathrm{ng} / \mathrm{ml}$ ciprofloxacin to identify clones that had incorporated the S83L substitution and lost the donor and mutagenesis plasmids. Successful mutagenesis was confirmed by Sanger sequencing.

Cultures were grown in Lysogeny broth/agar cultures (LB) (10 g/l tryptone, $5 \mathrm{~g} / 1$ yeast extract, $10 \mathrm{~g} / 1 \mathrm{NaCl}$; Bishop) at $37 \mathrm{C}$ throughout this study. LB media supplemented with $100 \mu \mathrm{g} / \mathrm{ml}$ ampicillin (Sigma-Aldrich) was used for plasmid 
maintenance. Susceptibility assays were performed using CIP (Sigma-Aldrich) at $100 \mathrm{ng} / \mathrm{ml}$.

\subsubsection{Randomized library construction}

The small RNA expression vectors pBad-DsrA, MicF, and Spot42 (Sharma et al. 2011; kindly donated by Yohei Yokobayashi, Okinawa Institute of Science and Technology) were used as templates for polymerase chain reaction (PCR) reactions. Randomized artificial sRNAs were constructed by incorporating random sequences in the antisense domains of sRNAs using PCR primers with degenerate bases. Platinum Pfx polymerase (Invitrogen) was used to PCR amplify the whole plasmid with a common reverse primer Sartrev containing 20 degenerate bases and an sRNA specific forward primer consisting of 10 degenerate bases for randomizing the antisense domain. The complete list of oligonucleotides used in this study is provided in supplementary Table B3.1.

The PCR amplicon was purified (Bio Basic) and digested using the DpnI enzyme (New England Biolabs) for 2 hours at $37 \mathrm{C}$ to cleave the methylated template parental DNA. Digested plasmids were phosphorylated using T4 polynucleotide kinase (New England Biolabs) for 20 minutes at 37 C. After phosphorylation, the linear DNA was self-ligated using T4 DNA ligase (New England Biolabs) and incubated at $25 \mathrm{C}$ overnight.

The consistency of the randomization protocol was first verified in Top10 cells. Subsequently, the randomized sRNA library was introduced into an E. coli gyrA S83L mutant by transforming chemically competent cells with the self-ligated PCR amplicons. 
Transformations were plated on LB agar supplemented with ampicillin, single colonies were picked, grown overnight at $37 \mathrm{C}$ at $150 \mathrm{rpm}$ with $100 \mu \mathrm{g} / \mathrm{ml}$ ampicillin, and glycerol stocks were prepared in a 96 well plate for further screening. Controls in each of the 96 well plates included gyrA S83L carrying empty sRNA plasmid and untransformed E. coli MG1655. Libraries were constructed separately for each of the three expression vectors, consisting of $\sim 5000$ transformants in total. $\sim 40$ plasmids from the randomized library (roughly equal numbers from each plasmid backbone) were extracted and sent for Sanger sequencing to confirm randomization.

\subsubsection{Ciprofloxacin sensitivity screen}

In order to identify sRNA constructs that increased CIP sensitivity, 24 hour growth curves in CIP-containing media were assayed for $\sim 5000$ gyr $\mathrm{S} 83 \mathrm{~L}$ mutant clones bearing randomized constructs. Cultures were inoculated at a 1:100 dilution from glycerol stock cultures and grown overnight at $37 \mathrm{C}, 150 \mathrm{rpm}$ in LB with $100 \mu \mathrm{g} / \mathrm{ml}$ ampicillin for sRNA plasmid maintenance. For 24-hour growth curves, the overnight cultures were diluted (1:100) in LB supplemented with $100 \mathrm{ng} / \mathrm{mL}$ CIP, $0.5 \mathrm{mM}$ arabinose, and $100 \mu \mathrm{g} / \mathrm{ml}$ ampicillin. Arabinose is required to induce expression of the sRNA construct. $100 \mathrm{ng} / \mathrm{mL}$ CIP was chosen as an intermediate concentration between the minimum inhibitory concentration (MIC) of the WT $(30 \mathrm{ng} / \mathrm{mL})$ and the S83L mutant $(600 \mathrm{ng} / \mathrm{mL})$. The $\mathrm{OD}_{600}$ of each culture was measured on a Biotek ELx808 plate reader every 37 minutes for 24 hours, incubating at $37 \mathrm{C}$ with 30 seconds of shaking every 5 
minutes. Two growth parameters, lag time, and maximum growth rate, were estimated using the program GrowthRates (Hall et al. 2013).

Clones showing growth repression in the primary CIP sensitivity screen were selected for secondary screening in the presence and absence of CIP $(100 \mathrm{ng} / \mathrm{ml})$ using similar experimental methods as described above. Clones whose growth was repressed in the presence of CIP, but not in LB alone, were selected for further analysis.

\subsubsection{Target identification}

In order to identify potential target mRNAs, sRNA vectors were extracted from CIPsensitive clones and the sRNA region was Sanger sequenced. Putative mRNA targets were identified for the randomized sRNA sequences using the online software packages IntaRNA (Mann et al. 2017), Target RNA (Kery et al. 2014), and RNA predator (Eggenhofer et al. 2011). These web based programs predict hybridizations between two RNA molecules, and provide a graphical overview of the sRNA-mRNA binding interactions (Tjaden 2008; Smith et al. 2010; Eggenhofer et al. 2011). The resulting target genes were identified and selected for experimental validation. 


\subsubsection{Validation for selected targets}

Candidate genes whose knockdown may induce CIP sensitization were validated experimentally by constructing double mutants, in which the gyrA S83L mutation was combined with knockout mutations of the computationally predicted target genes. The knockout mutants were selected from the Keio mutant collection (Baba et al. 2006), wherein a kanamycin resistance cassette was used to replace sequences of non-essential genes. The gyrA S83L mutation was introduced by oligonucleotide-mediated recombineering (Ellis et al. 2001) into the Keio deletion mutants, to construct each double mutant. A mobilizable derivative of pMA7SacB was used (Lennen et al. 2016), in which an arabinose-inducible promoter $\left(\mathrm{P}_{\mathrm{BAD}}\right)$ controls expression of the $\beta$ subunit of $\lambda$ Red recombinase and the E. coli Dam methylase. Dam methylase induction has been shown to increase mutagenesis efficiencies in E. coli by transiently interfering with the DNA mismatch repair system (Lennen et al. 2016). The plasmid was introduced into recipient strains by conjugation using a donor E. coli strain (WM3064) that is auxotrophic to diaminopimelic acid (DAP) (Dehio and Meyer 1997; Saltikov and Newman 2003). Recipients (Keio mutants) were cultured in LB broth supplemented with $30 \mu \mathrm{g} / \mathrm{ml}$ kanamycin and the donor was cultured in LB supplemented with $0.3 \mathrm{mM}$ DAP and $100 \mu \mathrm{g} / \mathrm{ml}$ ampicillin (for Red plasmid maintenance). Mating mixtures were spotted on LB agar with $0.3 \mathrm{mM}$ DAP and incubated overnight. Exconjugates were selected with $100 \mu \mathrm{g} / \mathrm{ml}$ ampicillin on LB lacking DAP, to prevent growth of donors. $\lambda$-Red recombination was performed on Keio mutants harboring the Red plasmid as previously described (Lennen et al. 2016) using an oligonucleotide encoding the gyrA (S83L) 
mutation $\left(5^{\prime}-\right.$

AACGCAGCGAGAATGGCTGCGCCATGCGGACGATCGTGTCATAGACCGCCÁA GTCACCATGGGGATGGTATTTACCGATTACGTCACCAA-3'). Transformants harboring the gyrA mutation were selected by plating on LB supplemented with $30 \mu \mathrm{g} / \mathrm{ml}$ CIP and $30 \mu \mathrm{g} / \mathrm{ml}$ kanamycin. The strains were cured of the $\lambda$ Red plasmid by $s a c B$ counterselection by streaking on LB agar containing 5\% sucrose. The gyr $A$ gene of each putative double mutant was PCR amplified and Sanger sequenced to verify the presence of the S83L mutation (PCR primers: Gyrase forward 5'GTAAAACGACGGCCAGTGATGAGCGAC3', Gyrase reverse 5'CGGTACGGTAAGCTTCTTC3').

\subsubsection{Ciprofloxacin susceptibility assay}

Minimum inhibitory concentrations (MIC) for CIP were determined for each of the gyrA S83L/ Keio knockout double mutants using a 96 well plate assay. Antibiotic concentrations were started at $125 \mathrm{ng} / \mathrm{ml}, 4 \mathrm{ug} / \mathrm{ml}$, and $8 \mathrm{ug} / \mathrm{ml}$ for Keio single knockout mutants, double mutants (Keio single knock out mutant + gyrA S83L), and controls $(c y b C$ only and $c y b C+g y r A \mathrm{~S} 83 \mathrm{~L})$ respectively, and were diluted in a two-fold series and dispensed with $125 \mu \mathrm{l} /$ well of LB into 96 -well plates. The 96 -well plates were incubated overnight at $37 \mathrm{C}$, with shaking at $150 \mathrm{rpm}$. The MIC was defined as the lowest concentration of antibiotic for which $90 \%$ growth inhibition was visibly observed after overnight culture. 


\subsection{Results}

\subsubsection{Randomized sRNA library construction}

We generated a library of artificial sRNAs by randomizing the antisense domain sequences of three native E. coli $\mathrm{sRNAs}$ : DsrA, MicF, and Spot42. We transformed the sRNA library into an E. coli gyrA S83L mutant and collected a total of $\sim 5000$ clones, each bearing a plasmid with an artificial sRNA construct (Figure 3.1A, B). We arbitrarily isolated 40 clones from the plasmid library and sequenced the sRNA regions to check for the incorporation of randomized sequences. The sequencing results confirmed the presence of unique randomized sequences in each of the engineered sRNA constructs (example sequences are shown in Table 3.1). 
Table 3.1 Randomized sequences of artificial sRNAs constructs. $\sim 10-30$ randomized bases (highlighted) were incorporated upstream of the scaffold regions of DsrA, MicF, and Spot42 by PCR amplification of source vectors lacking the antisense regions (“empty" vectors). All the sRNAs start from vector-derived sequence of 5 ACTCGAG (red).

\begin{tabular}{ll}
\hline \multicolumn{1}{c}{ Scaffold } & \multicolumn{1}{c}{ Randomized region } \\
\hline Wild-type DsrA sequence & actcgagcaatttttaagtgcttcttgcttaag \\
& actcgagttgtacctgctttcgatacgactttcatcaatttttaagtgcttcttgcttaag \\
Example randomized DsrA & actcgagttctctcgtcggactgaacgtggagctggaatttttaagtgcttcttgcttaag \\
Wild-type MicF sequence & actcgaggaattcggaccatgataccactgagtttaatttttaagtgcttcttgcttaag \\
Example randomized MicF & actcgagcgtcattcatttctgaatgtctg \\
& actcgagtcccttcacgggtgcaacgggccaatggcgtcattcatttctgaatgtctg \\
Wild-type Spot42 sequence & actcgagggtagtagactgcgtcattcatttctgaatgtctg \\
Example randomized Spot42 & actcgagggagggggggatttggctgaatattttagccgc \\
& actcgagccgtatagaaccacatctgcctgggggggatttggctgaatattttagccgc \\
\hline
\end{tabular}


(A)

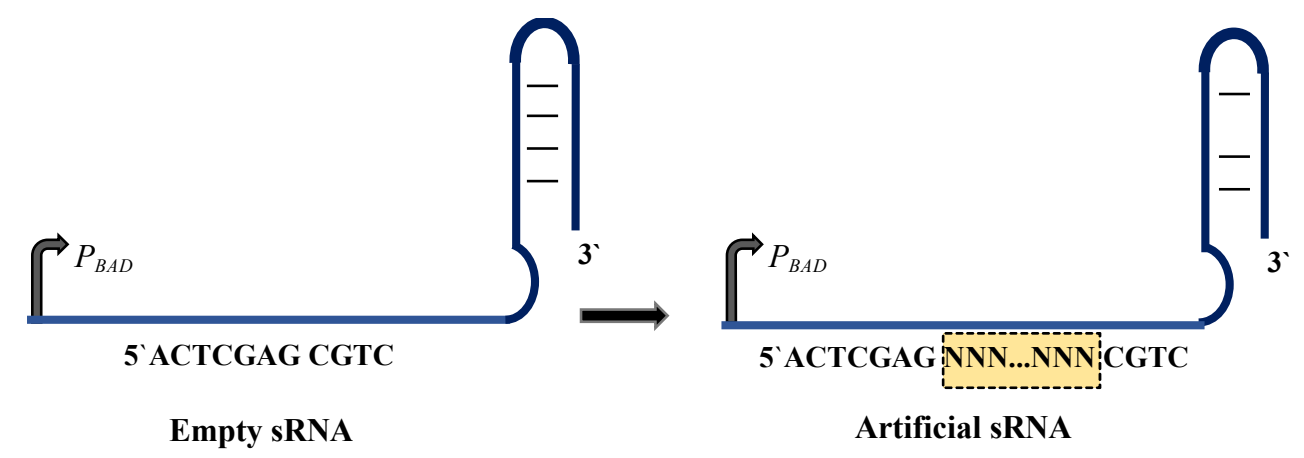

(B)

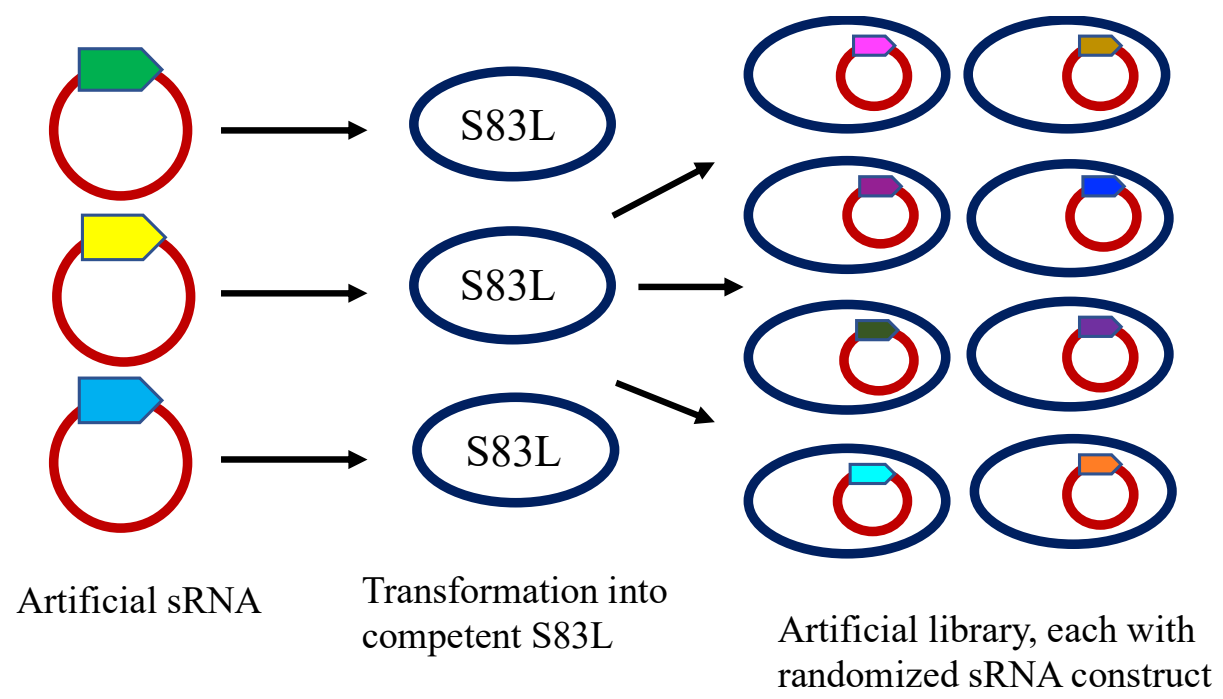

Figure 3.1 A schematic representation for the construction of the randomized artificial sRNA library. (A) The antisense domain is randomized with $\sim 10-30$ degenerate bases. The randomized bases were incorporated upstream of the scaffold regions of DsrA, MicF, and Spot42 by PCR amplification of source vectors lacking the antisense regions ("empty" sRNA). (B) The subsequent randomized product is transformed into a gyrA S83L mutant to obtain a randomized artificial library, with each clone bearing a novel sRNA construct. 


\subsubsection{Ciprofloxacin sensitivity screening}

To identify clones with increased susceptibility towards CIP, all $\sim 5000$ gyr $A$ S83L clones bearing sRNA constructs were grown in LB supplemented with 100ng/mL CIP and arabinose to induce sRNA expression. The MIC of the gyrA S83L mutant is $600 \mathrm{ng} / \mathrm{ml}$, so we reasoned that incubation at this sub-MIC concentration of CIP would be a good indicator for changes in sensitivity. Controls included wild-type E. coli MG1655 grown in LB without CIP (median growth rate: $0.09 \mathrm{OD}_{600} /$ minute and length of lag phase: 71 minutes), and a gyrA S83L mutant bearing a non-randomized plasmid grown in LB+CIP (median growth rate: $0.08 \mathrm{OD}_{600} /$ minute and length of lag phase: 250 minutes) (Figure 3.2). We selected a total of 528 clones exhibiting substantial reductions in growth rate and/or increased lag phase (outliers from Figure 3.2). From the individual sRNA scaffolds, we selected 199 clones from DsrA (median growth rate: $0.08 \mathrm{OD}_{600} /$ minute and length of lag phase: 395 minutes), 231 clones from MicF (median growth rate: 0.08 $\mathrm{OD}_{600} /$ minute and length of lag phase: 393 minutes), and 98 clones from Spot42 (median growth rate: $0.08 \mathrm{OD}_{600} /$ minute and length of lag phase: 275 minutes).

We repeated the growth curves of the 528 putatively CIP susceptible clones in the presence and absence of CIP (Figure 3.3, Supp Figure B3.1). This secondary screen was performed in order to verify the CIP-sensitive phenotypes and to determine whether or not the growth deficiencies were specific to CIP; a growth deficit in LB alone would indicate a general fitness effect of the sRNA, rather than reversal of CIP resistance. From this secondary screen, we selected 48 clones showing little or no growth inhibition in LB without CIP (Mean growth rate: $0.085 \mathrm{OD}_{600} /$ minute and length of lag phase: 478 
minutes), but whose growth was inhibited in LB supplemented with CIP (Mean growth rate: $0.071 \mathrm{OD}_{600} /$ minute and length of lag phase: 824 minutes). 


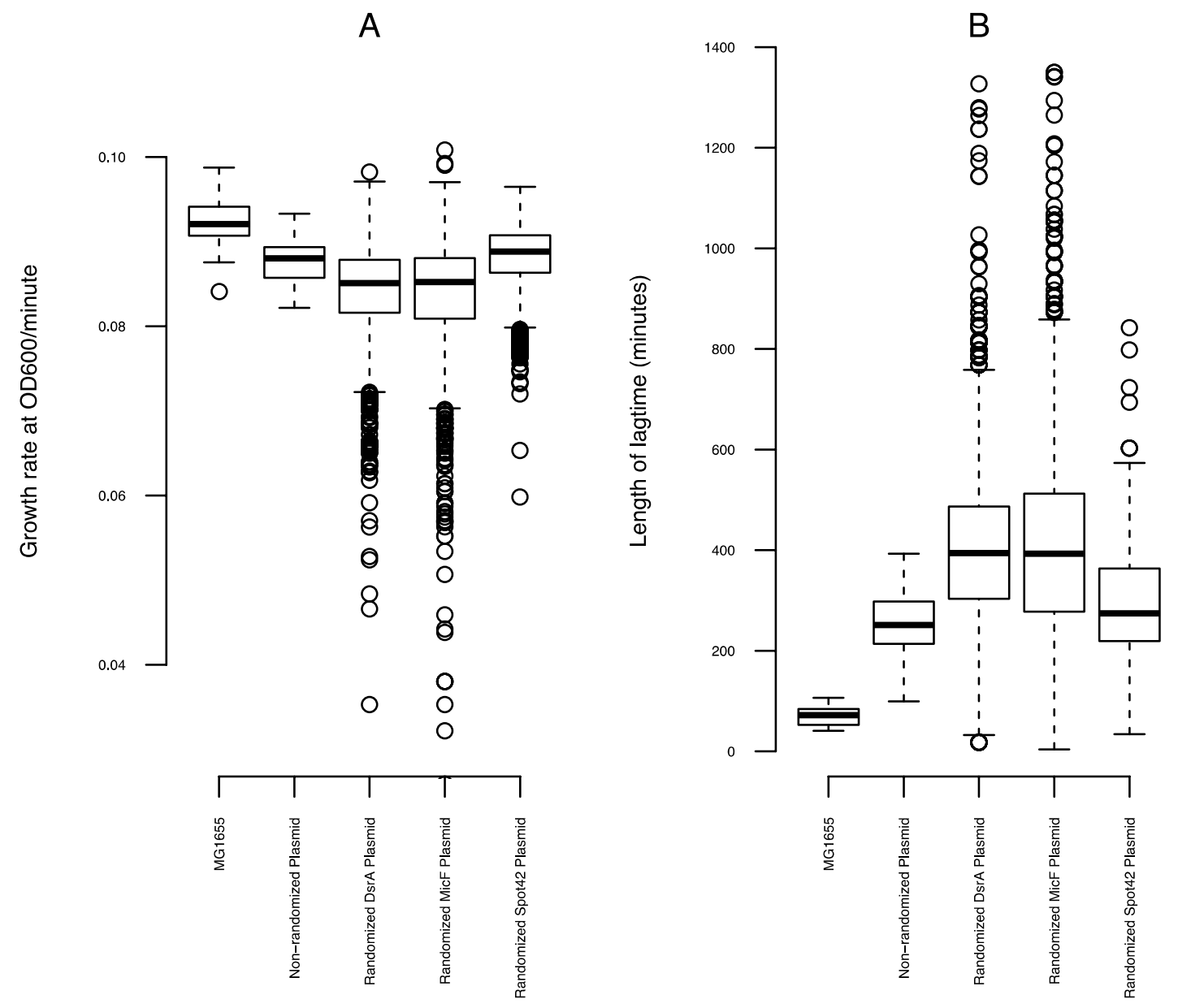

Figure 3.2 Boxplot distributions of CIP-sensitivity phenotypes for $\sim 5000$ gyrA S83L mutants harbouring randomized sRNA plasmid constructs. The growth rate (A) and lag time (B) distributions are depicted for clones cultured in LB with $600 \mathrm{ng} / \mathrm{ml} \mathrm{CIP.} \mathrm{E.} \mathrm{coli}$ MG1655 grown without CIP, and gyrA S83L mutants bearing empty sRNA plasmids grown with CIP, were used as controls. The boxplot presents the median, first, and third quartiles, with whiskers showing either the maximum (minimum) value or 1.5 times the interquartile range of the data, whichever is smaller (larger). The outliers (528 clones) selected for further investigation have substantial reductions in growth rate and/or increased lag phase. 


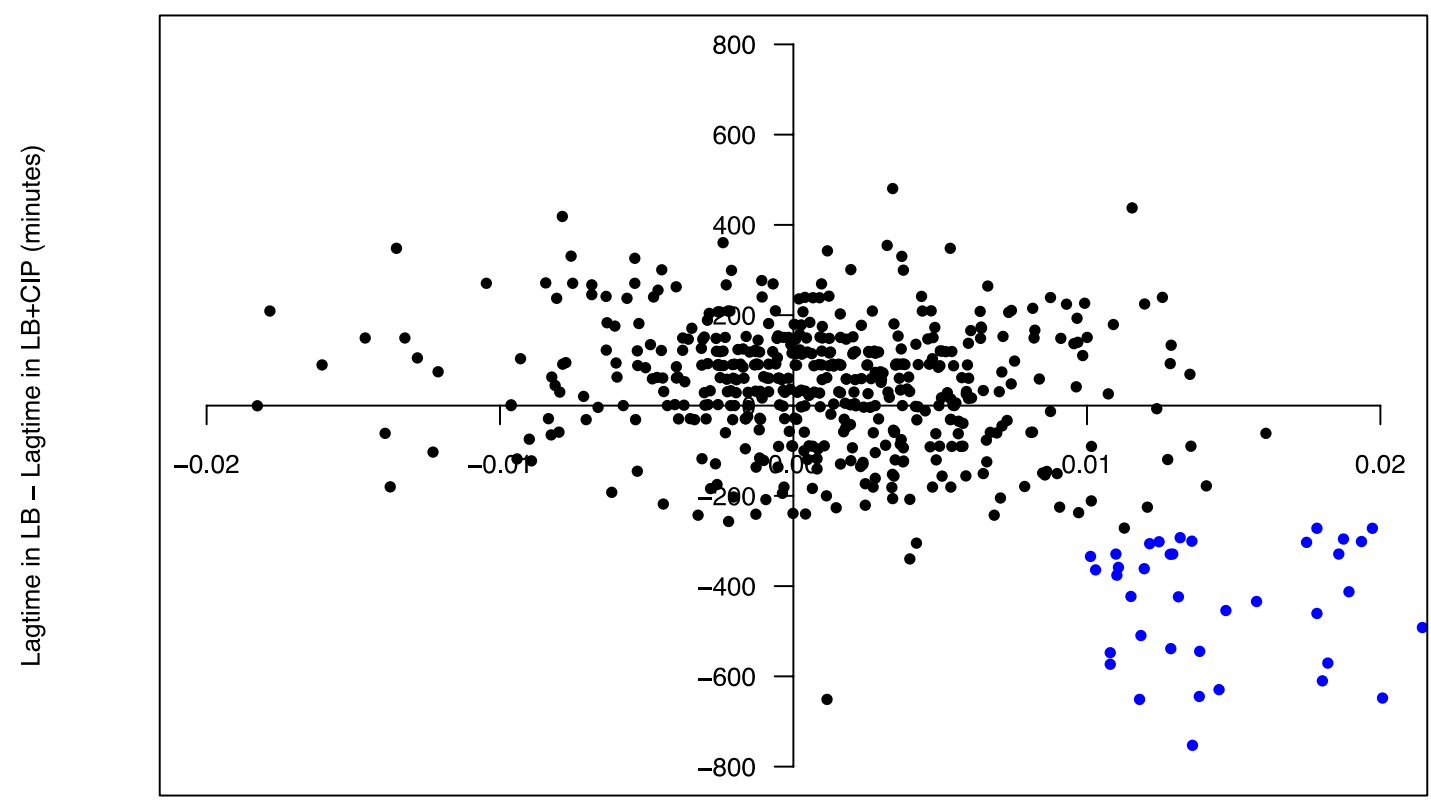

Growth rate in LB - Growth rate in LB+CIP (OD600/minute)

Figure 3.3 Effects of sRNA expression on growth rate $\left(\mathrm{OD}_{600} /\right.$ minute $)$ and length of lag phase (minutes) with and without CIP. The 48 selected constructs showing repression in growth in the presence of CIP are indicated in blue. 


\subsubsection{Identification of target genes}

We sequenced the randomized antisense regions of the sRNA plasmids isolated from the 48 clones exhibiting growth defects specific to the presence of CIP. Candidate target mRNAs were identified using the online tools IntaRNA (Mann et al. 2017), Target RNA (Kery et al. 2014), and RNA predator (Eggenhofer et al. 2011). These packages predict target mRNA sites in the E. coli MG1655 transcriptome with the potential for complementary sRNA interactions. We detected complementary hits for 31/48 randomized sequences (an example of complementarity between an artificial sRNA and its predicted target gene $(\mathrm{sbmC})$ is shown in Supp Figure B3.2). Since these tools identify complementary matches between short sequences, several sequences were matched to multiple targets, resulting in a total of 222 potential gene targets (Supp Table B3.2). Candidate targets included genes involved in DNA repair and recombination $(\mathrm{recC}$, $\mathrm{rec} D$, $r e c J, s b m C)$, the SOS response and error prone replication (umuC, xseA), transcriptional regulation $(y e e Y, m t l R)$, cell wall division and assembly $(z i p A, \min C)$, transport and efflux (emrB, tolQ), two-component regulatory systems ( $c p x A, c p x R, o m p R, c h e B)$, and chaperone proteins (hybB, dnaK).

\subsubsection{Experimental validation of targets}

We carried out validation experiments for 36 genes that encoded potential mRNA targets (Supp Table B3.3). Since CIP primarily interacts with DNA gyrase, we focused on putative target genes related to gyrase and its functions in DNA replication, repair and 
recombination. Additional genes were chosen because of their roles in efflux transport systems, two-component regulatory systems, transcriptional regulators and as chaperone proteins (Table 3.2). For each candidate target gene, the corresponding knockout mutant was obtained from the Keio single gene knockout collection (Baba et al. 2006). The gyrA S83L mutation was then transferred into the Keio clone by $\lambda$-Red mutagenesis, generating a double mutant.

To determine which candidate target genes conferred increased sensitivity to CIP on the gyrA S83L background, we obtained the MICs of each double mutant and the corresponding single mutants (Supp Table B3.4). Knockout of a pseudogene, $c y b C$, was used as a control for the effect of the kanamycin cassette present in the Keio clones. The MIC values of a number of double mutants showed reduced susceptibility when compared to the $c y b C+\mathrm{S} 83 \mathrm{~L}(\mathrm{MIC}=1000 \mathrm{ng} / \mathrm{ml})$ double mutant. Importantly, for 30 genes, the reduction in CIP resistance was more pronounced on the gyrA $\mathrm{S} 83 \mathrm{~L}$ background than on the WT background; these genes fall below the 1:1 line on Figure 3.4. Inactivation of these genes thus leads to substantial reductions in gyrA-mediated CIP resistance. Some of the mutants falling into this category include xseA, tus (double mutant MIC reduction of 16-fold), $s b m C$ (double mutant MIC reduction of 8-fold), tolQ and tolC (double mutant MIC reduction of 16-fold). 
Table 3.236 genes selected for experimental validation, encoding predicted sRNA targets associated with reductions in gyrA S83L mediated CIP resistance. (The locus tags indicate to the accession number, obtained from EcoCyc database collection (Ecocyc database: Keseler et al. 2017).

\begin{tabular}{|c|c|c|c|c|c|}
\hline S.NO. & $\begin{array}{l}\text { Target } \\
\text { gene }\end{array}$ & Function & Category & $\begin{array}{c}\text { Locus } \\
\text { tag }\end{array}$ & Reference \\
\hline 1 & $\mathrm{rec} C$ & $\begin{array}{l}\text { DNA helicase, ATP-dependent } \\
\text { dsDNA/ssDNA exonuclease }\end{array}$ & $\begin{array}{l}\text { DNA replication, } \\
\text { repair and } \\
\text { recombination }\end{array}$ & b2822 & $\begin{array}{l}\text { Persky and } \\
\text { Lovett (2008) }\end{array}$ \\
\hline 2 & $r e c D$ & $\begin{array}{l}\text { Exonuclease V (RecBCD } \\
\text { complex) alpha chain }\end{array}$ & $\begin{array}{l}\text { DNA replication, } \\
\text { repair and } \\
\text { recombination }\end{array}$ & b2819 & $\begin{array}{l}\text { Persky and } \\
\text { Lovett (2008) }\end{array}$ \\
\hline 3 & rec $J$ & $\begin{array}{l}\text { ssDNA exonuclease; 5' 3' } \\
\text { specific }\end{array}$ & $\begin{array}{l}\text { DNA replication, } \\
\text { repair and } \\
\text { recombination }\end{array}$ & b2892 & Han et al. (2006) \\
\hline 4 & rnt & Ribonuclease T (RNase T) & $\begin{array}{l}\text { DNA replication, } \\
\text { repair and } \\
\text { recombination }\end{array}$ & b1652 & $\begin{array}{l}\text { Viswanathan et } \\
\text { al. 1999) }\end{array}$ \\
\hline 5 & rpoN & $\begin{array}{l}\text { RNA polymerase sigma } 54 \\
(\text { sigma N) factor }\end{array}$ & $\begin{array}{l}\text { DNA replication, } \\
\text { repair and } \\
\text { recombination }\end{array}$ & b3202 & $\begin{array}{l}\text { Zhang et al. } \\
\text { (2016) }\end{array}$ \\
\hline 6 & $\operatorname{smr} B$ & Putative DNA endonuclease & $\begin{array}{l}\text { DNA replication, } \\
\text { repair and } \\
\text { recombination }\end{array}$ & b2331 & $\begin{array}{l}\text { Keseler et al. } \\
(2016)\end{array}$ \\
\hline 7 & $s b m C$ & DNA gyrase inhibitor & $\begin{array}{l}\text { DNA replication, } \\
\text { repair and } \\
\text { recombination }\end{array}$ & b2009 & $\begin{array}{l}\text { Nakanishi et al. } \\
\text { (1998) }\end{array}$ \\
\hline 8 & tus & $\begin{array}{l}\text { Inhibitor of replication at Ter; } \\
\text { DNA binding protein }\end{array}$ & $\begin{array}{l}\text { DNA replication, } \\
\text { repair and } \\
\text { recombination }\end{array}$ & b1610 & Hill et al. 1989 \\
\hline 9 & top $B$ & DNA topoisomerase III & $\begin{array}{l}\text { DNA replication, } \\
\text { repair and } \\
\text { recombination }\end{array}$ & b1763 & $\begin{array}{l}\text { DiGate et al. } \\
\text { (1988) }\end{array}$ \\
\hline 10 & итис & DNA polymerase $\mathrm{V}$; subunit $\mathrm{C}$ & $\begin{array}{l}\text { DNA replication, } \\
\text { repair and } \\
\text { recombination }\end{array}$ & b1184 & Tang et al. (1999) \\
\hline 11 & $u v r D$ & $\begin{array}{l}\text { DNA-dependent ATPase I and } \\
\text { helicase II }\end{array}$ & $\begin{array}{l}\text { DNA replication, } \\
\text { repair and } \\
\text { recombination }\end{array}$ & b3813 & $\begin{array}{l}\text { Matson and } \\
\text { Robertson (2006) }\end{array}$ \\
\hline 12 & $x s e A$ & Exonuclease VII large subunit & $\begin{array}{l}\text { DNA replication, } \\
\text { repair and } \\
\text { recombination }\end{array}$ & b2509 & $\begin{array}{l}\text { Vales et al. } \\
\text { (1982) }\end{array}$ \\
\hline 13 & $\operatorname{dna} Q$ & $\begin{array}{l}\text { DNA polymerase III epsilon } \\
\text { subunit }\end{array}$ & $\begin{array}{l}\text { DNA replication, } \\
\text { repair and } \\
\text { recombination }\end{array}$ & b0215 & $\begin{array}{l}\text { Echols et al. } \\
(1983)\end{array}$ \\
\hline 14 & helD & DNA helicase IV & $\begin{array}{l}\text { DNA replication, } \\
\text { repair and } \\
\text { recombination }\end{array}$ & b0962 & $\begin{array}{l}\text { Mendonca et al. } \\
\text { (1993) }\end{array}$ \\
\hline
\end{tabular}




\begin{tabular}{|c|c|c|c|c|c|}
\hline 15 & priB & Primosomal protein $\mathrm{N}$ & $\begin{array}{l}\text { DNA replication, } \\
\text { repair and } \\
\text { recombination }\end{array}$ & b4201 & $\begin{array}{l}\text { Heller and } \\
\text { Marians (2005) }\end{array}$ \\
\hline 16 & priC & $\begin{array}{l}\text { Primosomal replication protein } \\
\mathrm{N}\end{array}$ & $\begin{array}{l}\text { DNA replication, } \\
\text { repair and } \\
\text { recombination }\end{array}$ & b0467 & $\begin{array}{l}\text { Heller and } \\
\text { Marians (2005) }\end{array}$ \\
\hline 17 & $r n r$ & Exoribonuclease R; RNase R & $\begin{array}{l}\text { DNA replication, } \\
\text { repair and } \\
\text { recombination }\end{array}$ & b4179 & $\begin{array}{l}\text { Cheng and } \\
\text { Deutscher (2002) }\end{array}$ \\
\hline 18 & $u v r A$ & $\begin{array}{l}\text { ATPase and DNA damage } \\
\text { recognition protein of } \\
\text { nucleotide excision repair }\end{array}$ & $\begin{array}{l}\text { DNA replication, } \\
\text { repair and } \\
\text { recombination }\end{array}$ & b4058 & $\begin{array}{l}\text { Wagner et al. } \\
\text { (2009) }\end{array}$ \\
\hline 19 & yeeY & $\begin{array}{l}\text { Predicted DNA binding } \\
\text { transcriptional regulator }\end{array}$ & $\begin{array}{l}\text { Transcriptional } \\
\text { regulators }\end{array}$ & b2015 & $\begin{array}{l}\text { Keseler et al. } \\
\text { (2016) }\end{array}$ \\
\hline 20 & $m t l R$ & $\begin{array}{l}\text { Transcriptional repressor } \\
\text { (mannitol) }\end{array}$ & $\begin{array}{l}\text { Transcriptional } \\
\text { regulators }\end{array}$ & b3601 & $\begin{array}{l}\text { Figge et al. } \\
\text { (1994) }\end{array}$ \\
\hline 21 & $y d a G$ & Uncharacterized protein & $\begin{array}{l}\text { Uncharacterized } \\
\text { protein }\end{array}$ & b1355 & $\begin{array}{l}\text { Keseler et al. } \\
(2016)\end{array}$ \\
\hline 22 & $y d a V$ & $\begin{array}{l}\text { Uncharacterized protein } \\
\text { (predicted ATP binding } \\
\text { protein) }\end{array}$ & $\begin{array}{l}\text { Uncharacterized } \\
\text { protein }\end{array}$ & b1360 & $\begin{array}{l}\text { Hidese et al. } \\
\text { (2014) }\end{array}$ \\
\hline 23 & ompR & Response regulator with EnvZ & $\begin{array}{l}\text { Two component } \\
\text { regulatory system }\end{array}$ & b3405 & $\begin{array}{l}\text { Cai and Inouye } \\
\text { (2002) }\end{array}$ \\
\hline 24 & cheB & $\begin{array}{l}\text { Fused chemotaxis regulator } \\
\text { protein }\end{array}$ & $\begin{array}{l}\text { Two component } \\
\text { regulatory system }\end{array}$ & b1883 & $\begin{array}{l}\text { Baker et al. } \\
(2006)\end{array}$ \\
\hline 25 & $\operatorname{cpxA}$ & $\begin{array}{l}\text { Sensory histidine kinase with } \\
\text { CpxR }\end{array}$ & $\begin{array}{l}\text { Two component } \\
\text { regulatory system }\end{array}$ & b3911 & $\begin{array}{l}\text { Bury-Mone' et al. } \\
\text { (2009) }\end{array}$ \\
\hline 26 & $\operatorname{cpx} R$ & Response regulator with $\mathrm{CpxA}$ & $\begin{array}{l}\text { Two component } \\
\text { regulatory system }\end{array}$ & b3912 & $\begin{array}{l}\text { Batchelor et al. } \\
\text { (2005) }\end{array}$ \\
\hline 27 & $d n a K$ & $\begin{array}{l}\text { Chaperone Hsp } 70 \text {; co- } \\
\text { chaperone with DnaJ }\end{array}$ & Chaperons proteins & b0014 & $\begin{array}{l}\text { Mayer et al. } \\
(2000)\end{array}$ \\
\hline 28 & $h y b B$ & $\begin{array}{l}\text { Predicted hydrogenase } 2 \\
\text { cytochrome } b \text { type component }\end{array}$ & Chaperons proteins & b2995 & $\begin{array}{l}\text { Menon et al. } \\
\text { (1994) }\end{array}$ \\
\hline 29 & emrB & Multidrug efflux system protein & $\begin{array}{l}\text { Transport, Efflux } \\
\text { system }\end{array}$ & b2686 & $\begin{array}{l}\text { Lomovskaya et } \\
\text { al. (1995) }\end{array}$ \\
\hline 30 & tolA & $\begin{array}{l}\text { Membrane anchored protein in } \\
\text { TolA-TolQ-TolR complex }\end{array}$ & $\begin{array}{l}\text { Transport, Efflux } \\
\text { system }\end{array}$ & b0739 & $\begin{array}{l}\text { Bernadac et al. } \\
\text { (1998) }\end{array}$ \\
\hline 31 & tolQ & $\begin{array}{l}\text { Membrane spanning protein in } \\
\text { TolA-TolQ-TolR complex }\end{array}$ & $\begin{array}{l}\text { Transport, Efflux } \\
\text { system }\end{array}$ & b0737 & $\begin{array}{l}\text { Bernadac et al. } \\
\text { (1998) }\end{array}$ \\
\hline 32 & tolC & $\begin{array}{l}\text { AcrAB-TolC multidrug efflux } \\
\text { transport system }\end{array}$ & $\begin{array}{l}\text { Transport, Efflux } \\
\text { system }\end{array}$ & b3035 & $\begin{array}{l}\text { Bernadac et al. } \\
\text { (1998) }\end{array}$ \\
\hline 33 & $\min C$ & $\begin{array}{l}\text { Septum site determining } \\
\text { protein, inhibitor of FtsZ ring } \\
\text { polymerization }\end{array}$ & Cell division & b1176 & $\begin{array}{l}\text { Pichoff, and } \\
\text { Lutkenhaus } \\
\text { (2001) }\end{array}$ \\
\hline 34 & zipA & FtsZ stabilizer & Cell division & b2412 & $\begin{array}{l}\text { Hale and de Boer } \\
(2002)\end{array}$ \\
\hline 35 & proC & $\begin{array}{l}\text { Pyrroline-5-carboxylate } \\
\text { reductase NAD(P)-binding }\end{array}$ & $\begin{array}{l}\text { Catalytic, biosynthetic } \\
\text { pathways }\end{array}$ & b0386 & $\begin{array}{l}\text { Deutch et al. } \\
\text { (1982) }\end{array}$ \\
\hline 36 & pyr $B$ & Catalytic subunit & $\begin{array}{l}\text { Catalytic, biosynthetic } \\
\text { pathways }\end{array}$ & b4245 & $\begin{array}{l}\text { Donahue and } \\
\text { Turnbough } \\
\text { (1994) }\end{array}$ \\
\hline
\end{tabular}




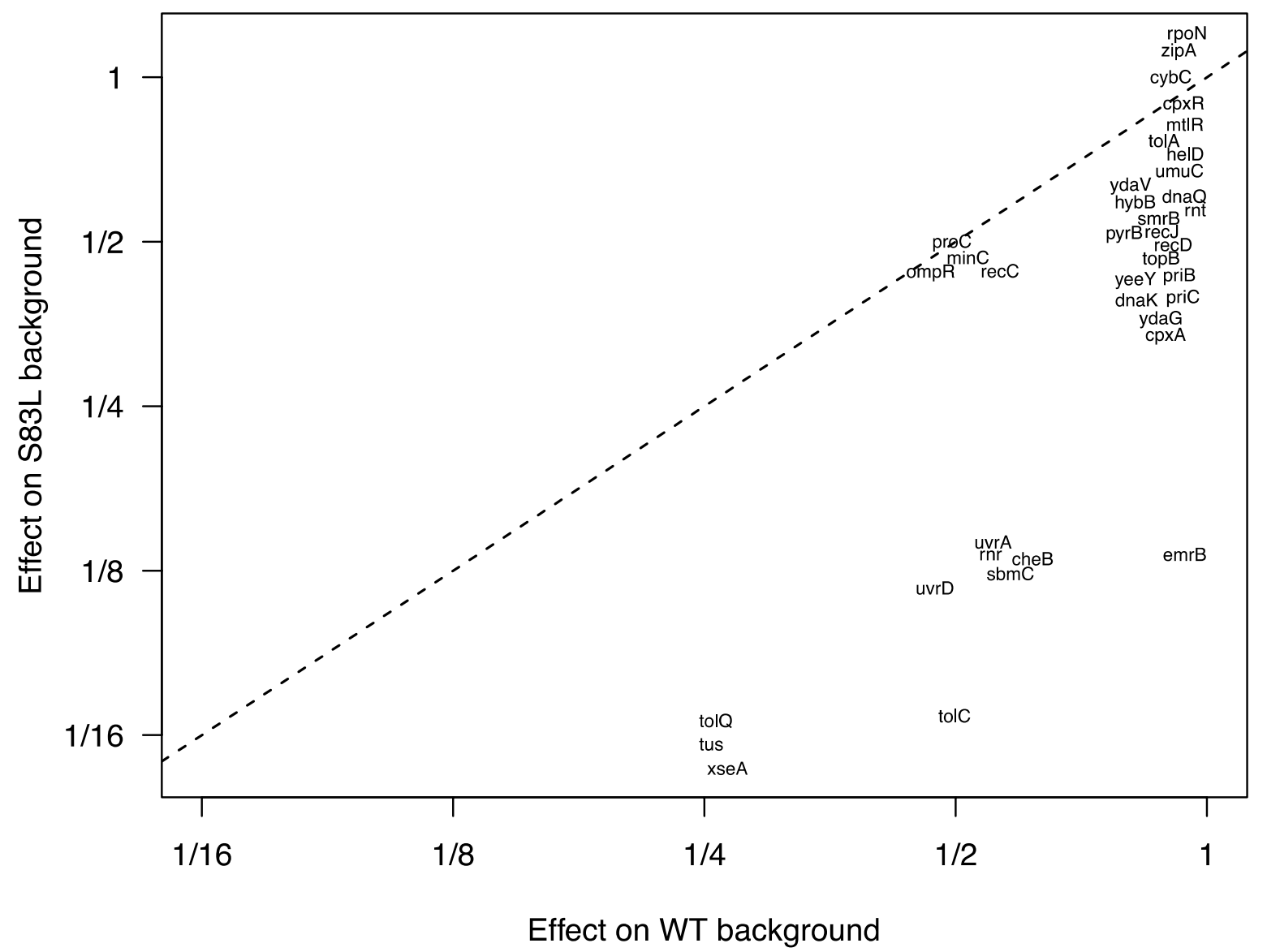

Figure 3.4 Fold change in MIC values for 36 knockout mutants on wild-type and gyrA S83L backgrounds. The 30/36 mutants that fall below the 1:1 line show reduction in CIP resistance on the gyrA S83L background but not on the wild-type background. 


\subsection{Discussion}

We carried out an sRNA screen to identify genes whose knockdown restores quinolone sensitivity in E. coli. Following an initial screen of $\sim 5000$ sRNA-bearing clones, and secondary screening of over 500 clones, we found 30 genes whose disruption increases sensitivity of a gyrA S83L mutant by 2 -fold or more. These genes have known functions in DNA repair and replication, drug efflux, and transcriptional regulation. Our findings expand knowledge of the genetic interaction network of the essential gene gyrA, and provide potential targets for the development of antibiotic adjuvants to restore sensitivity in quinolone resistant pathogens.

Chemical-genetic sensitivity screens have largely used knockout approaches, whereby a library of knockout mutants is screened for sensitivity or resistance to an antibiotic at sub-lethal concentrations (e.g., Tamae et al. 2008, reported 140, (approximately 3\%) out of 4000 Keio mutants against 7 antibiotics); Gomez and Neyfakh 2006; Breidenstein et al. 2008; Fajardo et al. 2008; Liu et al. 2010). Typically, such screens are carried out on a wild-type, antibiotic susceptible background, so they are not well-suited to identifying genes whose knockdown reverses resistance. Nonetheless, Tamae et al. (2008) did show that knockouts of five genes recovered from a sensitivity screen (recC, recA, fis, xseA, tolC; all but fis were also found here) did reduce CIP resistance on a gyrA mutant background. sRNA screens offer a powerful means for identifying genetic-background specific effects, since sRNA libraries can be readily 
generated in any transformable strain (Sharma et al. 2011, 2013; Lee et al. 2011). Moreover, inducible sRNA constructs can be used for studying gene functions of essential genes as well as non-essential genes (Rodrigo et al. 2012), which is not possible with knockouts. Our sRNA screen identified several candidate essential genes (gyrA and parC, encoding the targets of CIP) whose knockdown reduced growth in CIP. The randomization approach that we have adopted here (see also Sharma et al. 2011, 2013) is particularly promising, since it does not require targeted cloning of gene-specific sRNAs.

Direct use of sRNAs as therapeutics would require the efficient expression of a synthetic sRNA construct inside the target bacterial cell. This would require a competent delivery system, such as phage or a conjugative plasmid system. An engineered-phage approach illustrated by Lu and Collins (2009) expressed the LexA and OmpF proteins from phage M13. This resulted in reduction in antibiotic resistance evolution in mice when injected with engineered-phages. Thus, a comparable approach could be a plausible delivery system for combating resistant bacteria. An alternative strategy would be to identify small molecules that inhibit the targets identified here. For example, we showed that inhibition of $s b m C$ reduces CIP resistance by 2-fold, and SbmC is a known target of the peptide antibiotic Microcin B17 (Baquero et al. 1995; Collin et al. 2011). Thus, sRNA-mediated target discovery may be an efficient option for generating prospects for novel therapeutics. 


\title{
Chapter 4
}

\section{Two-component regulatory networks as determinants of stress responses and antimicrobial resistance in}

\author{
Escherichia coli
}

\subsection{Abstract}

Two-component signal transduction systems (TCS) serve as stimulus-response mechanisms that allow bacteria to monitor diverse environmental cues. The typical bacterial TCS consists of a transmembrane sensor kinase and a cytoplasmic response regulator, regulating physiological responses to a variety of environmental parameters, including phenotypic resistance due to various stressors. The present study aims to elucidate the physiological basis of bacterial tolerance in response to different envelope stresses using well elucidated TCSs that act as global regulators of envelope stress tolerance, as well as antibiotic resistance. Here, I have characterized the responses of eight mutants from four TCSs of Escherichia coli to combinations of external stressors and antibiotic challenges. I identified TCS mutants exhibiting different phenotypic 
changes in response to stresses, antibiotics and stressor+antibiotic combinations. While the stressor-only conditions generally slowed growth rates of the TCS mutants, increased growth rates were observed under some stressor+antibiotic combination. Similarly, TCS mutants showed gain in resistance in the presence of stressor, with reductions in resistance levels in its absence. Overall, this work broadens the understanding of the mechanisms by which $E$. coli responds to the external physiological environment, including antibiotics. 


\subsection{Introduction}

The tolerance of bacterial pathogens to fluctuating environmental challenges is based on their rapid repertoire of responses to distinct environments. Environmental stresses that perturb bacterial cell envelope integrity can impact its growth and survival (Poole et al. 2012). It is of great interest, therefore, to understand bacterial survival strategies in the landscape of membrane stress. In both Gram-positive and Gram-negative bacteria, twocomponent signal transduction systems (TCSs) play a pivotal role in regulating diverse protective stress responses (Stock et al. 1989; Hoch and Silhavy 1995; 2000; Gao and Stock 2009). Recognition of specific environmental cues/signals that perturb the cell envelope's integrity, and interpretation of this information through modulating transcriptional responses, is the essence of such TCSs (Stock et al. 2000; Mascher et al. 2006; Laub et al. 2007). TCSs have been shown to respond to a variety of environmental signals, such as osmolarity, antibiotics, and nutrient availability (Parkinson 1993; Hoch and Silhavy 1995; Mizuno 1997; Oshima et al. 2002; Krell et al. 2010). TCSs regulate a range of activities, such as sporulation, biofilm formation, chemotaxis, nutrient utilization, virulence to antimicrobial susceptibility, and resistance (Mizuno 1997; Stock et al. 2000).

TCSs are comprised of two protein modules: a transmembrane receptor or sensor histidine protein kinase $(\mathrm{HK})$ protein, and its cognate, intracellular signalling receiver known as a response regulator (RR) (Stock et al. 2000). The various HKs and RRs constitute a complex signal transduction system, culminating in the regulation of gene 
expression. Concisely, after sensing an external stimulus (e.g., ligand binding) the HK undergoes a conformational change and as a result auto-phosphorylates at a conserved histidine $(\mathrm{H})$ residue using of the $\gamma$-phosphate of ATP. Subsequently, the signal information as a phosphoryl moiety is transferred to a conserved aspartate residue of a cognate RR, triggering the expression of a large number of target genes (Stock et al. 2000; Gao and Stock 2009; Zschiedrich et al. 2016) (Figure 4.1).

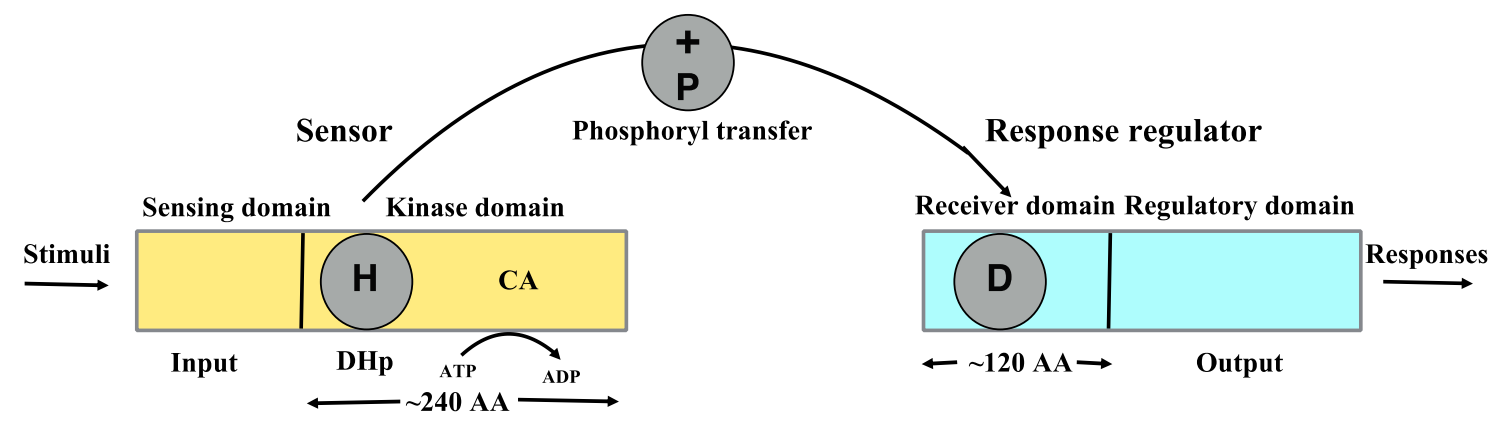

Figure 4.1 Schematic of a stereotypical two-component signal transduction system's (TCS) phosphorylation cascade. The sensor domain responds to a stimulus and uses ATP to autophosphorylate at conserved histidine $(\mathrm{H})$ residue, located at the kinase core domain. The phosphoryl group $(\mathrm{P})$ is transferred to a conserved aspartate $(\mathrm{D})$ residue of its cognate response regulator, which subsequently triggers the output response. 
In Escherichia coli, $\sim 30$ TCSs have been identified by experimental evidence and protein sequence similarity. For comparison, this is roughly half the number of TCSs found in Pseudomonas aeruginosa (Rodrigue et al. 2000; Gooderham and Hancock 2009). Mizuno et al. (1997) used the sequences of OmpR, NarL, NtrC, and CheY as probes to explore E. coli TCSs with similar open reading frames. The members of each TCS family show strong amino acid conservation of domains involved in signal transduction, reflecting functional similarities (Supp Figure C4.1). For example, in the OmpR family of TCS, the phosphotransfer, ATP-domain, and regulator domain have maintained common sequences and structure (Gross et al. 1989; Hoch 2000).

In Gram-negative bacteria, many TCSs respond to envelope stresses triggered by different stressors. In the context of antimicrobial resistance (AMR) TCSs act as global regulators of AMR as they optimize bacterial metabolism based on their surrounding environmental challenges (such as envelope stress responses) and thereby impact levels of resistance (Tamae et al. 2008; Fajardo et al. 2008; Martinez et al. 2009; Dam et al. 2018). Given that antimicrobial compounds target components of the cell envelope or penetrate the cell envelope to gain entry to their respective targets, it is not surprising that membrane permeability impacts antimicrobial susceptibility (Hirakawa et al. 2003; Pagès et al. 2008; Fernandez and Hancock 2012; Poole 2014). TCSs also act as regulators, and thus participate in regulating the expression of many genes leading to effects on efflux pumps, cell envelope components, and antibiotic-degrading enzymes (Lee et al. 2009). TCSs modulate antibiotic susceptibility through their global regulatory phosphorelay networks that regulate bacterial physiology while adjusting to different environmental cues. Here, I study several well elucidated TCSs that are global regulators of envelope 
stress and that impact antimicrobial susceptibility in E. coli: PhoQ-PhoP, EnvZ-OmpR, CpxA-CpxR, and BaeS-BaeR .

The PhoP-PhoQ (Pho) TCS is linked to virulence and cationic antimicrobial peptide (CAMP) resistance in several Enterobacteriaceae, P. aeruginosa, and Salmonella typhimurium (Kato and Groisman 2008; Gooderham and Hancock 2009). The Pho system facilitates the response to limiting magnesium concentrations and affects antimicrobial susceptibility against aminoglycosides and membrane disrupting CAMPs (e.g., polymyxin B and colistin) (Macfarlane et al. 2000; Cheng et al. 2010; Zschiedrich et al. 2016). This is primarily mediated by regulating the genes involved in the production of lipid A of the bacterial lipopolysaccharide (LPS) layer (Macfarlane et al. 2000;

Gooderham and Hancock 2009; Needham and Trent 2013; Dalebroux and Miller 2013; Ram and Goulian 2013). The Pho system also regulates other features of bacterial metabolism, such as glycogen metabolism of E. coli (Montero et al. 2009) as well as several other genes involved in mediating bacterial metabolism (Santos-Beneit 2015).

Another relevant TCS is EnvZ-OmpR, widely found in E. coli and other Gramnegative bacteria. This system responds to changes of medium osmolarity and plays a role in iron homeostasis, synthesis of flagella, and amino acid metabolism (Forst and Roberts 1994; Cai and Inouye 2002). The EnvZ-OmpR phosphorelay system regulates these bacterial physiological effects, in part, through transcriptional regulation of two major outer membrane proteins, OmpF and OmpC (Forst and Roberts 1994; Yamamoto et al. 2005; Yoshida et al. 2006). As porins are the gateway for the entry of small ßlactams and other hydrophilic antibiotics, such as tetracycline, chloramphenicol and 
fluoroquinolones, a decrease in porin-mediated permeability caused by any loss-offunction/regulatory mutations in the porins confers high level resistance to antibiotics (Nikaido 2003; Doumith et al. 2009; Källman et al. 2009).

Similarly, in E. coli and other Gram negative organisms, the CpxR-CpxA (Cpx) system contributes to multidrug resistance (MDR) (Hu et al. 2011; Audrain et al. 2013; Kurabayashi et al. 2014; Surmann et al. 2016). The Cpx system is predominantly involved in the envelope stress maintenance system and responds to a variety of environmental stresses including bactericidal antibiotics that generate misfolded envelope proteins and impact the cell envelope adversely (Raffa and Raivio 2002; Ruiz and Silhavy 2005; Raivio 2014; Delhaye et al. 2016). In E. coli, the Cpx system upregulates the transcription of the marRAB operon. This operon activates TolC-dependent tripartite MDR efflux transporters, which in turn facilitates resistance to aminoglycosides, $\beta$ lactams, novobiocin, and CAMPs (Audrain et al. 2013; Mahoney and Silhavy 2013; Raivio 2014; Weatherspoon-Griffin et al. 2014; Delhaye et al. 2016). Girgis et al. (2009) in their transposon-mutagenized library of E. coli determined the involvement of Cpx system in governing intrinsic antibiotic susceptibility.

The BaeR-BaeS (Bae) TCS also contributes to MDR by modulating the expression of multidrug transporters in E. coli (Nishino and Yamaguchi 2001; Baranova and Nikaido 2002; Nishino and Yamaguchi 2002; Leblanc et al. 2011). The Bae system responds to envelope stress and is known to confer resistance to novobiocin and $\beta$ lactams by upregulating Bae-dependent $m d t A B C D$ and $a c r D$ MDR efflux transporters (Baranova and Nikaido 2002; Hirakawa et al. 2003; Bury-Moné et al. 2009; Leblanc et 
al. 2011; Rosner and Martin 2013). Lin et al. (2013) identified the Bae system in Acetobacter baumannii, and showed that baeSR mutants are susceptible to tigecycline. The Bae system also confers resistance to variety of other antimicrobials, including aminoglycosides, tetracycline, rifampicin, and fluoroquinolones (Hu et al. 2011; Guerrero et al. 2013).

In this study, I have selected eight mutants from four TCSs (Pho, EnvZ-OmpR, $\mathrm{Cpx}$, and $\mathrm{Bae}$ ) that are the global regulators of stress tolerance and antimicrobial resistance in E. coli. I have obtained TCS mutants from the Keio single-gene knockout mutant collection and exposed them to a stand-alone stressor $\left(\mathrm{NaCl}\right.$ and $\left.\mathrm{MgCl}_{2}\right)$ and in a combination of stressor plus antibiotic conditions. These clinically relevant antibiotics belong to different antibiotic classes- ciprofloxacin (CIP), ceftazidime (CEF), tetracycline (TET), and chloramphenicol (CHM). In normal metabolic regulation, upon sensing environmental stress, TCSs get activated. Therefore, if a particular TCS is involved in the response to a particular stress condition, presumably we can expect a TCS mutant to display a decreased growth phenotype in the presence of that particular stress condition. Similarly, in a stressor+antibiotic encounter, TCS mutants are expected to show a different growth phenotype, providing direct evidence that these mutants play an important role in the tolerance of stressful conditions, as well as antibiotics. Accordingly, the profiling of $E$. coli responses to envelope stresses has expanded our understanding of intricate control systems in elucidating the potential link between complex TCS, and their impact on growth upon exposure to a particular antibiotic. 


\subsection{Materials and Methods}

\subsubsection{E. coli strains}

Eight mutants from four TCSs (Pho, EnvZ-OmpR, Cpx, and Bae) were selected from the Keio single-gene knockout collection (Baba et al. 2006). The Keio collection was derived from the wild-type strain K-12 BW25113 (lacI ${ }^{+} r r n B{ }_{\mathrm{T} 14} \Delta \operatorname{lac}_{\mathrm{WJ} 16} h s d R 514$ $\left.\triangle a r a B A D_{\mathrm{AH} 33} \Delta r h a B A D_{\mathrm{LD} 78}\right)$. Each of the 3985 strains in the Keio collection carries a complete deletion of a different gene with a kanamycin resistance cassette replacing the gene of interest. Throughout this experiment, I have used a knockout of $c y b C$, which is a pseudogene, as a control for the effect of the kanamycin resistance cassette present in the Keio clones.

\subsubsection{Media and culture conditions}

M9 minimal media (M9 MM) (composition per liter (Bioshop): 6.78g Na2HPO4, 3.00g $\mathrm{KH} 2 \mathrm{PO} 4,0.50 \mathrm{~g} \mathrm{NaCl}, 1.00 \mathrm{~g} \mathrm{NH} 4 \mathrm{Cl}, 0.24 \mathrm{~g} \mathrm{MgSO} 4,0.01 \mathrm{~g} \mathrm{CaCl} 2,8.00 \mathrm{~g}$ glucose as the carbon source, $16 \mathrm{~g}$ agar, $\mathrm{pH} 7$ ) was used for agar and broth cultures. When needed, the growth medium was supplemented with inducers and antibiotics. The antibiotics ciprofloxacin, ceftazidime, tetracycline, and chloramphenicol were used (Sigma-Aldrich) in this study at their minimum inhibitory concentrations (MIC) (Andrews 2001): ciprofloxacin $(0.015 \mu \mathrm{g} / \mathrm{ml})$, ceftazidime $(0.06 \mu \mathrm{g} / \mathrm{ml})$, tetracycline $(1 \mu \mathrm{g} / \mathrm{ml})$, and 
chloramphenicol $(2 \mu \mathrm{g} / \mathrm{ml})$. The chemicals that were used as inducers in this study and their concentrations are: $\mathrm{MgCl}_{2}(200 \mu \mathrm{M}$, Bioshop) and $\mathrm{NaCl}(0.5 \mathrm{M}$, Bioshop) (Supp Table C4.1).

\subsubsection{The growth of TCS mutant strains in M9 MM +/- stress conditions on solid medium}

Each of the TCS mutants was transferred from the frozen glycerol Keio collection stock into $5 \mathrm{ml}$ of M9 MM with $0.8 \%(\mathrm{w} / \mathrm{v})$ glucose as the sole carbon source and grown

overnight on a rotary shaker at $37 \mathrm{C}, 150 \mathrm{rpm}$ (optimal growth conditions). The overnight culture was inoculated into a fresh 150ul of M9 MM in a 96-well plate at a 1:500 dilution and grown overnight on a rotary shaker at $37 \mathrm{C}, 150 \mathrm{rpm}$. The high dilution minimizes nutrient leftovers from the overnight culture.

For colony size measurements the overnight cultures were used and spotted in triplicate on rectangular plates (single well with lid $(86 \times 128 \mathrm{~mm})$, Thermo fisher scientific) having M9 MM with $0.8 \%$ glucose and $1.5 \%$ agar (using a pinning tool (V\&P scientific Inc.(408FP6)). Where required, the stressor $+/-$ antibiotic were pre-added into the solid media at the same concentrations as mentioned above. All combinations of stressor and antibiotic were used, including M9 MM alone, M9 MM + stressor, M9 MM + antibiotic, and M9 MM + stressor + antibiotic. Overnight cultures were spotted at the centre of the plates in order to avoid errors in colony quantification, since colonies on the periphery are oversized compared to interior colonies. All plates were then incubated at 
$37 \mathrm{C}$ overnight. High resolution images of each plate were obtained using the ProtoCOL 3 instrument (Synbiosis, MD, USA) with an ultra-HD 5 megapixel scientific grade camera. Colony size was measured using two automated image analysis software packages, available online, SGA tools and the R package gitter image (http://bioconductor.org/biocLite.R). These tools quantify growth by obtaining an image of the plate and measuring pixel counts of the colony within a defined boundary.

\subsubsection{4-hour growth curve analysis}

The growth of each TCS mutant in broth culture was estimated using 24-hour growth curves using three independent replicates. Growth curves were obtained in 96-well plates, inoculated at a 1:100 dilution from overnight cultures in a 96-well plate with fresh M9 MM. Again, all four possible combinations of stressor and antibiotic were used, including $\mathrm{M} 9 \mathrm{MM}+$ no antibiotic, $\mathrm{M} 9 \mathrm{MM}+$ stressor, $\mathrm{M} 9 \mathrm{MM}+$ antibiotic, and M9 MM + stressor + antibiotic. Absorbance $\left(\mathrm{OD}_{600}\right)$ was measured on a Biotek ELx808 plate reader every 37 minutes for 24 hours, incubating at $37 \mathrm{C}$ with 30 seconds of shaking every 5 minutes. Two growth parameters, lag time, and maximum growth rate after 24 hours, were estimated using the program GrowthRates (Hall et al. 2013). 


\subsubsection{Antibiotic susceptibility assay}

Minimum inhibitory concentration (MIC) values were determined for all of the TCS mutants involved in this study using +/- stressor conditions in a 96 well plate assay. Antibiotic concentrations were diluted in a two-fold series and dispensed with $125 \mu \mathrm{l} /$ well of M9 MM into 96-well plates. The 96 well plates were incubated overnight at $30 \mathrm{C}$, with shaking at $150 \mathrm{rpm}$. The MIC was defined as the lowest concentration of antibiotic for which $90 \%$ growth inhibition was visibly observed after overnight culture. The MIC value was determined in triplicates with similar results. 


\subsection{Results and Discussion}

I systematically examined four TCSs that are involved in E. coli's response to envelope stresses, such as $\mathrm{NaCl}$ and $\mathrm{MgCl}_{2}$, and their impact on growth upon confronting a particular antibiotic. These TCSs are also involved in conferring intrinsic resistance to $E$. coli due to the low permeability of its outer membrane, regulating expression of efflux pumps and porins. To study these TCSs, I used knockout mutants from the Keio single gene knockout collection (Baba et al. 2006). For each mutant, I measured (1) growth rate parameters in M9 MM under four treatments (a) M9 MM as control (b) MM with a stressor (c) MM + antibiotic (d) MM + stressor + antibiotic, and (2) antibiotic susceptibility under stressor+/-antibiotic conditions. I have chosen stressors (sodium and magnesium) to match known functions of the TCS genes; some of the stressors are common to more than one TCS mutant. I have chosen four antibiotics from different drug classes with different modes of antimicrobial action (CIP, CEF, TET, and CHM) (Supp Table C4.2). As a control, a pseudogene $c y b C$ is used under all the growth parameters. Overall, each of the eight different TCS mutants exhibited compromised growth rates in comparison to $c y b C$ while growing in M9 MM without any treatments (no stressor, no antibiotic) (Anova: $\mathrm{F}=57.59, \mathrm{P}=6.06 \mathrm{e}-08^{* * *}$ ). The different responses of TCS mutants to different treatments, i.e. antibiotics and stressor+antibiotic, combinations are discussed below. 


\subsubsection{The impact of stressors on TCS mutants}

Each of the mutants performed worse with stressor than without stressor, whereas the control $c y b C$ does not (Figures 4.2-4.5, blue and red lines under no drug) (Table 4.1). This finding includes $c p x A$ and $c p x R$, envZ, ompR, baeS, baeR under a sodium stressor and $p h o Q$, phoP in the presence of magnesium. These TCSs are known to contribute to membrane architecture, suggesting a plausible explanation for decline in growth under the selected stressor conditions.

Table 4.1 A two-way analysis of variance (ANOVA) for the effects of stressor and mutants. (Stars indicate significance: ${ }^{* * *} \mathrm{p}<0.001$ ).

\begin{tabular}{ccc}
\hline Factor & F-value & $\boldsymbol{P}$-value \\
\hline Mutant & 274 & $2.05 \mathrm{e}-05 * * *$ \\
Stressor & 274 & $4.76 \mathrm{e}-08 * * *$ \\
Mutant * Stressor & 0.183 & 0.987 \\
\hline
\end{tabular}




\subsubsection{The impact of antibiotic on TCS mutants}

To investigate the role of these TCS genes in antibiotic susceptibility in E. coli, I selected four clinically relevant antibiotics (CIP, CEF, TET, and CHM) from different drug classes. I measured the growth rates of TCS mutants at the MIC of each drug. Overall, I observed that each of the mutants showed significantly reduced growth in the presence of any of the four antibiotics tested, compared to the control $c y b C$ knockout and irrespective of the presence of the stressor (Figures 4.2-4.5 red line) (Table 4.2). Thus, each of the antibiotics impacted growth rates of all the mutants significantly indicating a decreased tolerance to these antibiotics. This is not surprising given the involvement of these genes in efflux systems, porin-mediated permeability, and LPS modification.

Table 4.2 A two-way analysis of variance (ANOVA) for the effects of antibiotic and mutants. (Stars indicate significance: ${ }^{* * *} \mathrm{p}<0.001$ ).

\begin{tabular}{ccc}
\hline Antibiotic & F-value & $\boldsymbol{P}$-value \\
\hline CIP & 337.4 & $<2 \mathrm{e}-16^{* * *}$ \\
CEF & 229.9 & $<2 \mathrm{e}-16^{* * *}$ \\
TET & 184.1 & $1.16 \mathrm{e}-15^{* * *}$ \\
CHM & 266.9 & $<2 \mathrm{e}-16^{* * *}$ \\
\hline
\end{tabular}




\subsubsection{The response of stressor+antibiotic combinations on TCS mutants}

Each of the TCS mutants tested here showed significantly compromised growth rates compared to the control $c y b C$ knockout in a stressor+antibiotic combination (Table 4.3). As each of the TCSs are activated and regulate a set of different genes to cope with the environmental stresses, a TCS mutant is expected to confer impairment in its assigned function.

In the presence of stressor+antibiotic, envZ and ompR exhibited lower growth rates in comparison to the antibiotic-only treatment. Surprisingly, the mutants baeS, baeR, cpxA, cpxR, phoQ, and $p h o P$ showed increased growth rates compared to the antibiotic-only treatment (compare the blue and red lines in Figures 4.2-4.5). Intriguingly, this suggests that the presence of stressor in a medium can increase a mutant's tolerance to antibiotics in some mutants. One possible explanation for the improved tolerance of some TCS mutants under antibiotic+stress conditions is that some of the regulated genes are common between different TCSs. For example, the Cpx and Bae systems can regulate the expression of the same genes in relieving envelope stress, triggered by limited magnesium concentrations. These genes are $m d t A B C D$ (a multidrug efflux pump), spy (a periplasmic protein), and $a c r D$ (a predicted membrane facilitator) (Raffa and Raivio 2002; Baranova and Nikaido 2002; Zhau et al. 2003; Bury-Moné et al. 2009; Leblanc et al. 2011). Because of this redundancy, I speculate that the Bae system is upregulating these genes in Cpx mutants, and vice versa. By contrast, EnvZ-OmpR is the main regulatory system that controls permeability of the outer membrane by regulating the two major porins of E. coli (OmpC and $\mathrm{OmpF})$. The extent to which each porin is expressed 
predominantly depends on the phosphorylation state of OmpR when set by EnvZ. OmpR can be cross-phosphorylated by other TCSs (such as Cpx or Bae), but in that case only negligible phosphorylation of OmpR is observed (Skerker et al. 2005; Siryaporn and Goulian 2008; Bury-Moné et al. 2009). This lack of redundancy may therefore explain the decreased growth rates observed in $e n v Z$ and $o m p R$ mutants under stressor+antibiotic conditions.

Table 4.3 Effect of mutants on the growth rate (OD600/minute) parameter. (Stars indicate significance: ${ }^{*} \mathrm{p}<0.05,{ }^{* *} \mathrm{p}<0.01$, and $\left.{ }^{* * *} \mathrm{p}<0.001\right)$.

\begin{tabular}{|c|c|c|}
\hline \multirow[t]{2}{*}{ Factor } & $\begin{array}{l}\text { Post ho } \\
\text { with }\end{array}$ & $\begin{array}{l}\text { SD) comparisons of mutants } \\
\text { under stressor+antibiotic } \\
\text { treatment } \\
\text { ate }\left(\mathrm{OD}_{600} / \text { minute) }\right.\end{array}$ \\
\hline & F value & $P$-value \\
\hline$c y b C-p h o Q$ & 16.97 & $0.00208 * *$ \\
\hline$c y b C-p h o P$ & 6.051 & $0.0337 *$ \\
\hline$c y b C$-env $Z$ & 53.72 & $2.51 \mathrm{e}-05^{* * *}$ \\
\hline$c y b C$-отрR & 11.07 & $0.00766^{* *}$ \\
\hline$c y b C-c p x A$ & 6.976 & $0.0247 *$ \\
\hline$c y b C-c p x R$ & 23.73 & $0.00065 * * *$ \\
\hline$c y b C-b a e S$ & 9.505 & $0.0116 *$ \\
\hline$c y b C-b a e R$ & 7.408 & $0.0215 *$ \\
\hline
\end{tabular}



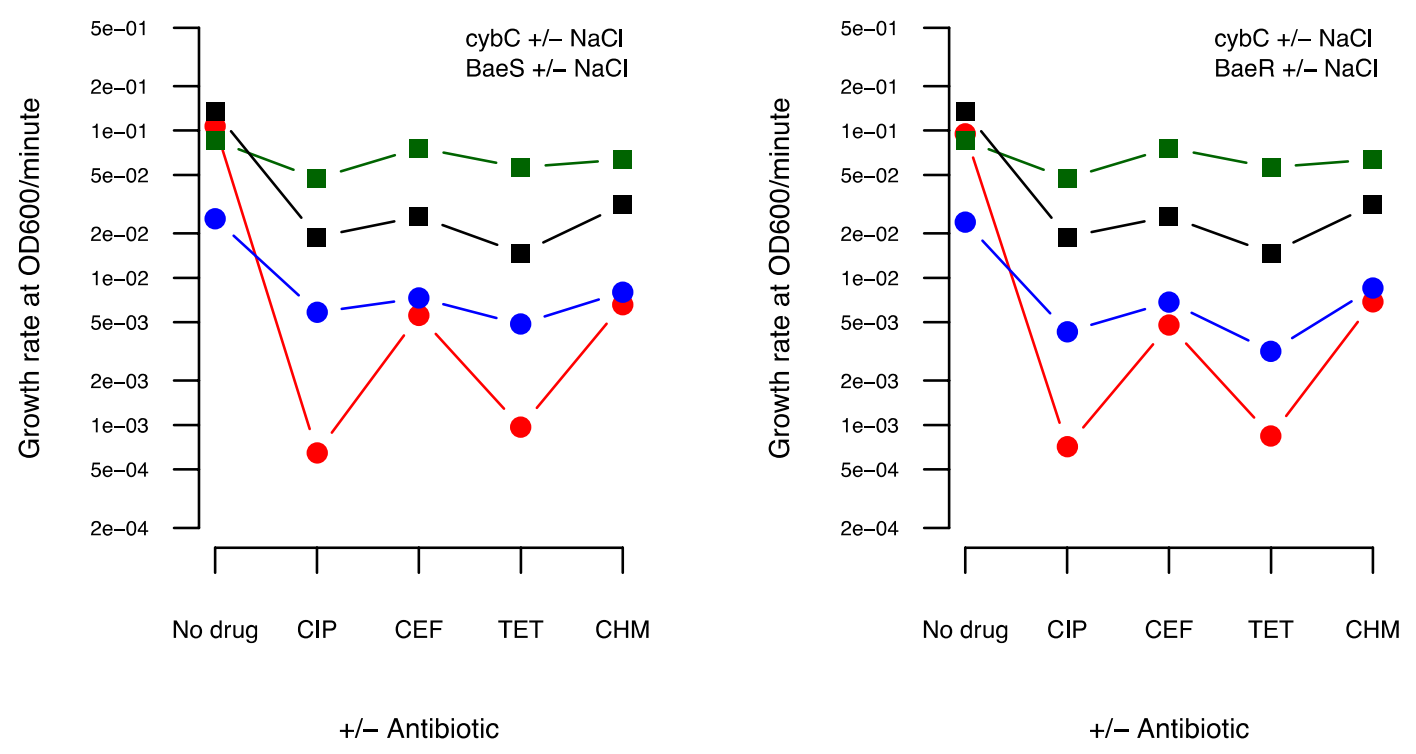

Figure 4.2 Growth rate of baeS and baeR mutants in the presence and absence of stressor $(\mathrm{NaCl})$. The red line represents no stressor and blue with stressor condition. The $c y b C$ control can be seen without stressor (black line) and with stressor (green line). (Each point represents the average of three independent biological replicates). 

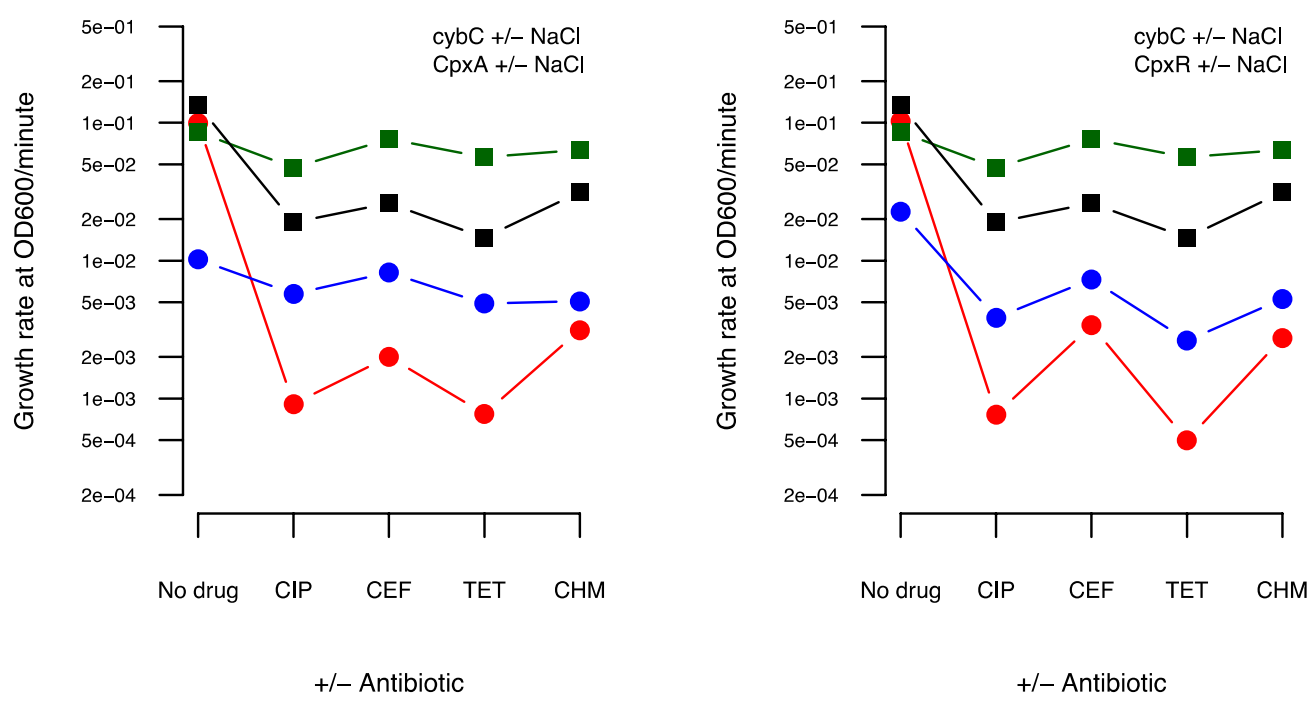

Figure 4.3 Growth rate of $c p x A$ and $c p x R$ mutants in the presence and absence of stressor $(\mathrm{NaCl})$. The red line represents no stressor and blue with stressor condition. The $c y b C$ control can be seen without stressor (black line) and with stressor (green line). (Each point represents the average of three independent biological replicates). 

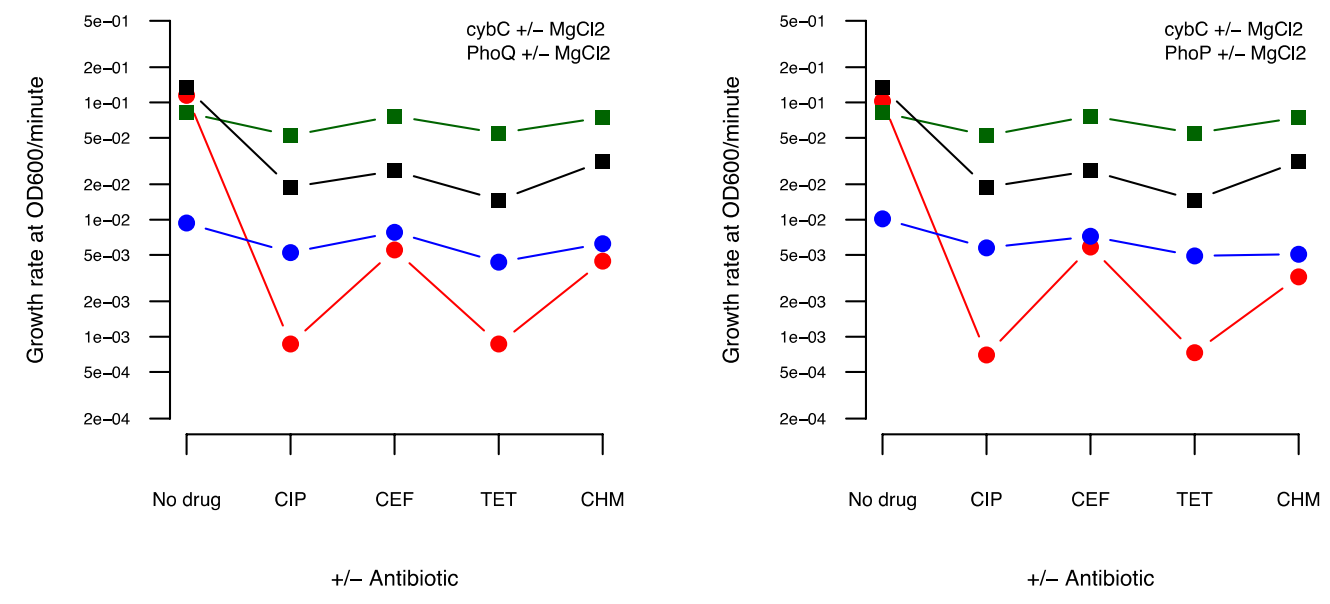

Figure 4.4 Growth rate of $p h o Q$ and $p h o P$ mutants in the presence and absence of stressor $\left(\mathrm{MgCl}_{2}\right)$. The red line represents no stressor and blue with stressor condition. The $c y b C$ control can be seen without stressor (black line) and with stressor (green line). (Each point represents the average of three independent biological replicates) 

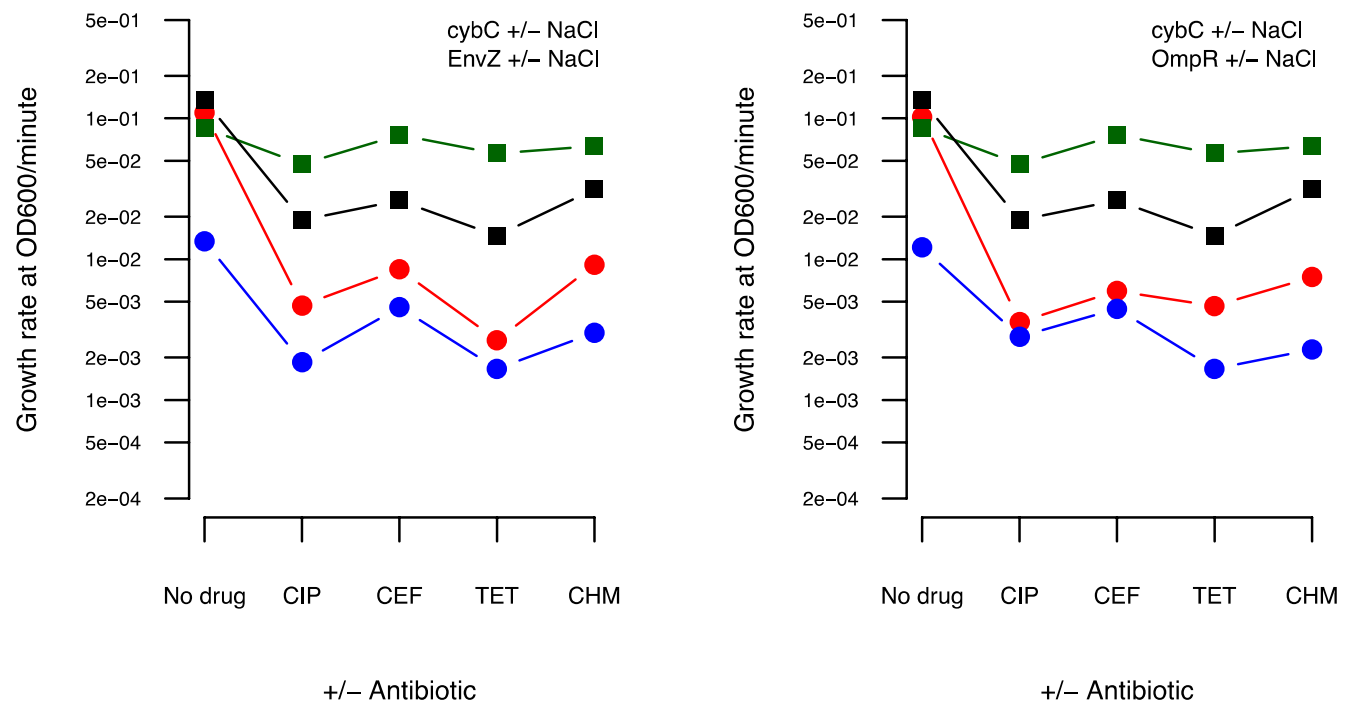

Figure 4.5 Growth rate of env $Z$ and $\operatorname{omp} R$ mutants in the presence and absence of stressor $(\mathrm{NaCl})$. The red line represents no stressor and blue with stressor condition. The $c y b C$ control can be seen without stressor (black line) and with stressor (green line). (Each point represents the average of three independent biological replicates). 


\subsubsection{Antibiotic susceptibility}

I quantified the MICs of all the eight mutants (baeS, baeR, cpxA, cpxR, phoQ, phoP, $e n v Z$, and $\operatorname{ompR}$ ) against all four antibiotics, with and without stressor. Overall, mutants in the presence of stressor showed either equal or increased resistance levels of 1-3 fold compared to the no-stressor condition, while comparable differences were not seen for the $c y b C$ mutant control (Figure 4.6 A, B, C, D). Two mechanisms may underlie this result. First, improved resistance levels may be induced by the envelope stress response through TCS-mutant independent mechanisms. Second, while experiencing a stressful encounter, different TCSs may operate as a proxy for one another, with many of the same genes regulated by these different systems. For example, the Cpx and Bae TCSs have synergistic effects in response to envelope stress (Raffa and Raivio 2002; Dorel et al. 2006; Rosner and Martin 2013). The Pho system is also linked to other TCSs (such as Rcs or Evg TCSs) that can form a cross-regulatory network and are implicated in a coordinated synthesis/modification of cell envelope through the activation of common transporters, such as MgtA, MgtB, MgrB (magnesium transporters) and SafA (a transmembrane protein) in response to the status of external magnesium (Kato et al. 1999; Groisman et al. 2001; Hagiwara et al. 2004; Eguchi et al. 2007; Moon and Gottesman 2009). However, I observed that envZ and ompR mutants showed decreased resistance levels for the four antibiotics tested, suggesting that EnvZ-OmpR is essential for regulating and maintaining bacterial envelope stress tolerance and porin synthesis.

In the absence of stressor, each of the TCS mutants showed a reduction in levels of resistance to all four antibiotics, ranging from 2-4 fold, whereas no changes were 
observed for the $c y b C$ knockout control (Figure 4.6 A, B, C, D and Supp Table C4.3). A 2-fold decrease in the MICs of CIP and CEF and a 4-fold decrease in the MIC of TET and CHM can be observed for both envZ and ompR mutants compared with that of the control $c y b C$ (Figure 4.6B). Other mutants, such as $c p x R$ and $c p x A$, also showed increased sensitivity to the antibiotics tested (Figure 4.6C). These results are in agreement with other studies describing the effects of these mutants on intrinsic resistance to various antibiotics. Tamae et al. (2008) and Liu et al. (2010) also found in genome-wide sensitivity screens that mutants of $e n v Z$ and $o m p R$ were susceptible to one or more of the antibiotics tested. 

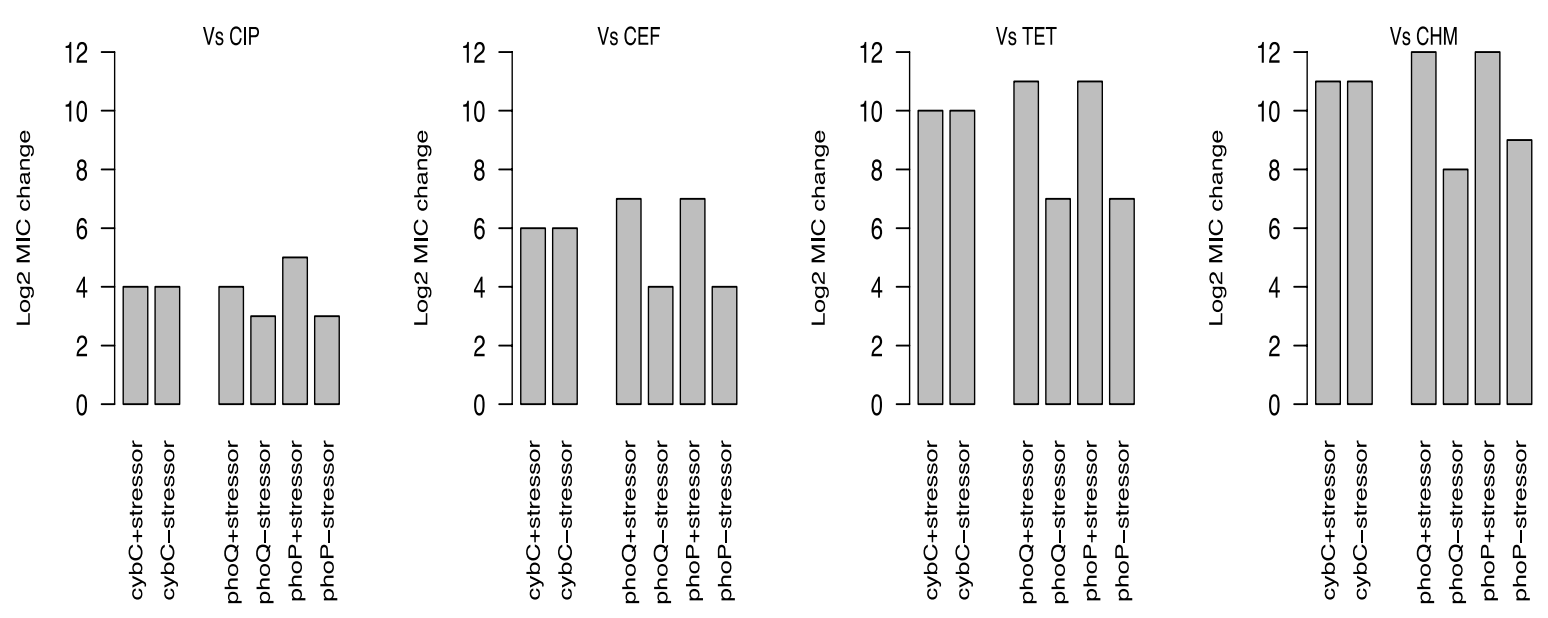

Figure 4.6A $\log _{2} \mathrm{MIC}$ change of phoQ, phoP TCS mutants under stressor $\left(\mathrm{MgCl}_{2}\right)+/-$ antibiotic conditions compared to control $c y b C$.

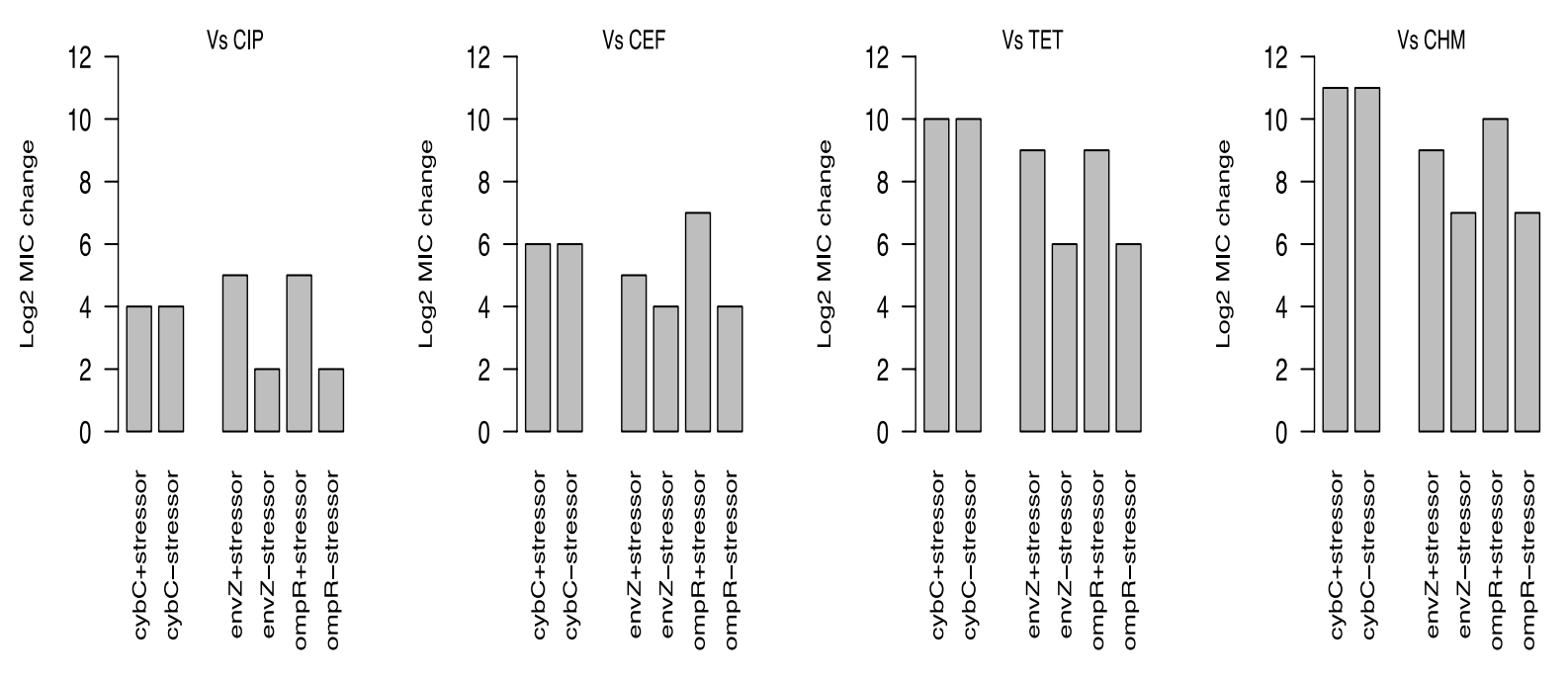

Figure 4.6B $\log _{2} \mathrm{MIC}$ change of envZ, ompR TCS mutants under stressor $(\mathrm{NaCl})+/$ antibiotic conditions compared to control $c y b C$. 

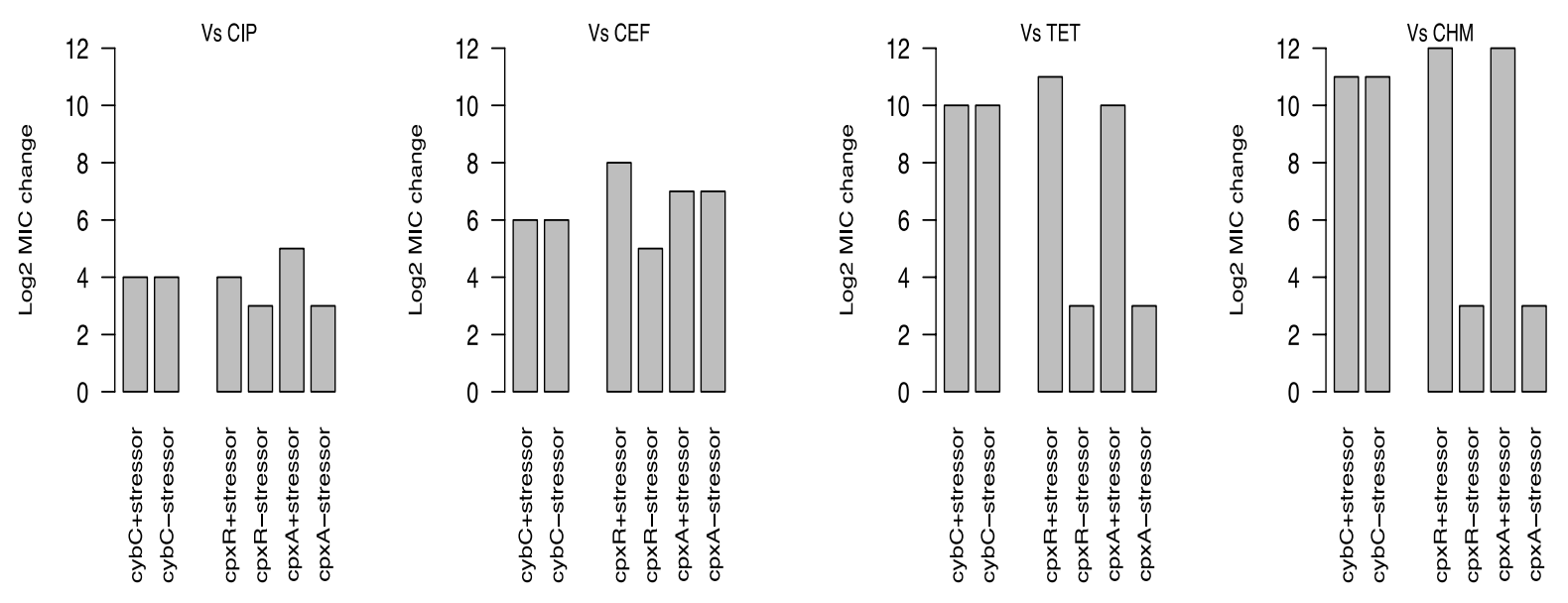

Figure 4.6C $\log _{2}$ MIC change of cpxR, cpxA TCS mutants under stressor $(\mathrm{NaCl})+/$ antibiotic conditions compared to control $c y b C$.
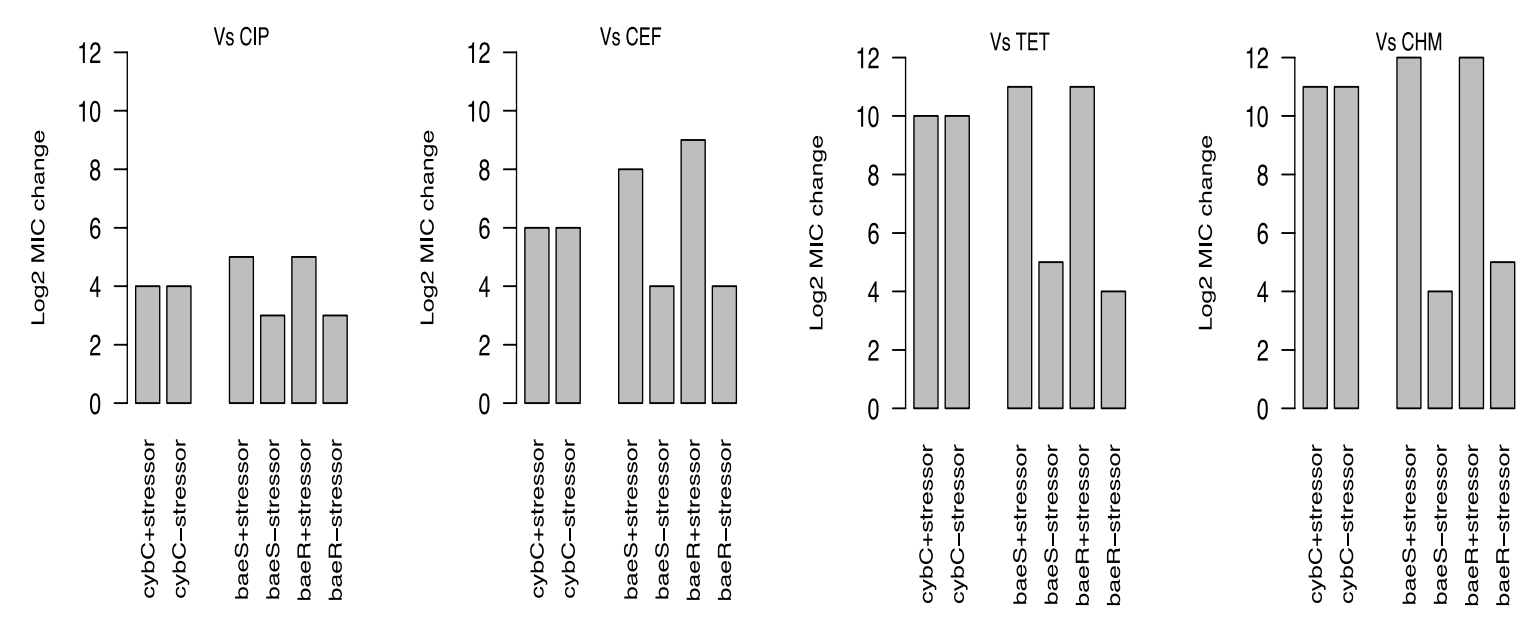

Figure 4.6D $\log _{2}$ MIC change of baeR, baeS TCS mutants under stressor $(\mathrm{NaCl})+/-$ antibiotic conditions compared to control $c y b C$. 
I carried out agar growth assays under similar conditions as the liquid growth assays, and results were broadly similar to the liquid growth assay (Supp Table C4.4, C4.5, Supp Figure C4.2, C4.3). As observed here, colony sizes varied significantly among all the treatments compared to control. 


\subsection{Conclusions and Perspectives}

TCSs are one of the predominant signaling regulatory families that are used by a bacterium to rapidly cope with changes in its environment. TCSs can impact bacterial performance considerably. The current study is an effort to gain more insight into the role of E. coli TCSs in conferring phenotypic resistance upon confronting metabolic stresses. I identified eight mutants from four TCSs, which upon exposure to a combination of stresses +/- antibiotic could show different growth phenotypic responses. As such, in the present study I found that $e n v Z$ and $o m p R$ mutants showed increased sensitivity to all four antibiotics tested, and to the presence of stress+antibiotic combination as well. The other six mutants showed different phenotypic responses in $+/$ - stress and/or antibiotic conditions. This study illustrates that the efficacy of TCSs is likely to depend on the environment it confronts, which suggests that the presence of stress in a medium can actually increase or decrease growth capacity as well as tolerance to antibiotics of some TCS mutants.

Bacterial tolerance pathways are upregulated to increase survival upon confronting a stressful encounter, and when these responses impact antibiotic activity, they have the potential to contribute to resistance. These transient, reversible resistance gains are linked to the physiological state of a bacterium upon confronting different habitats or stressors. For example, Allison et al. (2011) took advantage of bacterial metabolic states to increase susceptibility to aminoglycosides by combating persister cells by priming their metabolism. Similarly, biofilm-forming microorganisms display 
different metabolic states, where actively growing cells are killed by antibiotics and the fitness of remaining persister cells is dependent on nutrient availability, regulated by TCSs (Corona and Martinez 2013; Olivares et al. 2013; Zhang et al. 2013).

TCSs contribute to stress regulation associated with clinically relevant AMR, for example cell envelope stress-responsive TCS targets associated with efflux systems and LPS modification in Gram-negative organisms (Fraud and Poole 2010; Poole 2012; Poole et al. 2014). Due to their effects on AMR, virulence, and survival, TCSs have emerged as potential targets for therapeutic intervention. Lee et al. (2009) showed that the inactivation of the AmgRS envelope stress TCS in P. aeruginosa increases susceptibility to aminoglycosides in clinical settings. Similarly, Marchand et al. (2004) showed that the inactivation of adeRS resulted in improved aminoglycoside effectiveness in Acinetobacter baumanni, and inactivation of the SOS response that is dependent upon oxidative stress renders cells more susceptible to fluoroquinolones by decreasing fluoroquinolone stimulated persister formation by E. coli (Dörr et al. 2009). Many synthetic compounds are also explored that can inhibit TCSs, affecting bacterial growth and virulence (Barrett et al. 1998; Barrett and Hoch 1998; Macielag and Goldschmidt 2000; Stephenson and Hoch 2002; Gotoh et al. 2010; Harris et al. 2013). Rasko et al. (2008) have reported a small molecule that inhibits the sensor HK QseC in Salmonella typhimurium, lowering the virulence of several pathogens in animal studies without killing the bacterial cells. Watanabe et al. (2012) discovered a novel antibacterial Singermycin B, that targets the walKR TCS to inhibit bacterial growth in MDR bacteria including methicillin-resistant $S$. aureus. 
Despite a growing appreciation of the importance of TCSs to bacterial physiology, a complete understanding of TCS function, and how the TCS network integrates signals and stimuli in an orderly manner, has yet to be achieved. TCSs are not just linear on/off switches, but there is emerging concern about the complexity of the bacterial signaling networks (Olivera et al. 2010; Capra and Laub 2012; Jung et al. 2012; Guckes et al. 2013; Salazar and Laub 2015; Breland et al. 2017). These complexities include cross-interactions between closely related TCSs in a way that can be beneficial for the bacteria, such as a gain in antimicrobial resistance (Kraxenberger et al. 2012; Fried et al. 2013; Behr et al. 2014; Guckes et al. 2017; Urano et al. 2017; Wei et al. 2017). Therefore, cross regulation may pose a problem as any particular set of genes regulated by a specific TCS could also be under the control of other systems, and thus the effect of inhibiting a given component may not be observed if the functions of this system can be complemented by other systems. This present study also highlights one aspect of TCS, i.e. how bacteria respond to stresses that are encountered through environmental changes. In order to recognize the complete potential of TCS as stress responses and AMR determinants, more insight is required. 


\section{Chapter 5}

\section{Concluding Remarks}

Antimicrobial resistance (AMR) is one of the most pressing issues of recent times. Multidrug resistance in bacterial pathogens not only causes harm by jeopardizing medical treatments but also imposes a major economic burden. A growing gap between clinical need and antibiotic innovation has aggravated the crisis. Therefore, it is becoming increasingly challenging to find new efficacious antibiotics and novel therapeutic strategies to combat the accelerating resistance problem. This thesis provides and expands our understanding of both the evolution of AMR and approaches to counteract the resistance problem once it has arisen. In this section, I will discuss the significance of the findings presented in this work within the context of tackling the problems of AMR.

In chapter 2 of my thesis, I aim to achieve comprehensive insights about the rates, molecular bases, and pleiotropic consequences of resistance evolution. I obtained 56 spontaneous resistance mutants using fluctuation analysis across three generations of quinolones in Escherichia coli. I found 36 gyr A, 4 gyrB, 9 marR, and 1 double gyrA/ marR mutants, with two novel gyrB mutations. Overall, gyrA mutations conferred higher resistance, without greater fitness cost than other mutations, granting a selective advantage. I found widespread cross-resistance amongst quinolone resistant isolates, implying that different resistance mutations respond differently to quinolone variants. 
The results obtained in this chapter have implications for public health strategies, notably in antibiotic selection. The precise predictions about the rate and trajectories of resistance evolution towards new and old generations of antibiotics provide possibilities to take appropriate measures for the use of antimicrobials. In addition, as costs of resistance drive the evolution and persistence of resistance genes, a quantitative understanding of these costs and their effects is useful for optimizing treatment regimens and in managing resistance. As suggested in the current study of quinolones, the selection of levofloxacin would be ideal, since resistance is costly and single mutations have considerably smaller effects.

While it is imperative to develop new antimicrobials, improvements in antibiotic adjuvants are also essential. New strategies to preserve already existing antibiotics, for example using adjuvants, is one promising contribution. The efficacy of $\beta$-lactamase inhibitors over the past decades demonstrates the effectiveness of adjuvant concept (Drawz and Bonomo 2010; King et al. 2014). To help counteract the resistance problem, in chapter 3, I have used a novel approach for genetic screens in E. coli. Using a small RNA (sRNA) screen I have identified potential drug targets for the re-sensitization of quinolone resistant $E$. coli. Screening of $\sim 5000$ sRNA constructs on a gyrA mutant background led to the identification of 30 auxiliary drug targets. These genes are involved in DNA replication, repair and recombination (18), transport and efflux system (4), two-component regulatory system (4), transcriptional regulators (2), chaperone proteins (2), cell division (2) catalytic, biosynthetic pathways (2), and uncharacterized proteins (2). The repression of these genes reduced resistance to the fluoroquinolone ciprofloxacin in a gyrA S83L mutant. This screening approach has the potential to 
establish a promising future for combating AMR and can be used for various already existing antibiotics and different microorganisms.

Finally, I examined the involvement of E. coli's two-component signal transduction systems (TCS) in countering multiple stresses in chapter 4. I used eight TCS mutants from the Keio single gene knock-out collection. I examined the growth phenotype of each mutated TCS to different environmental stress conditions in combination with four antibiotics from different classes. Overall, the mutants showed different phenotypic changes in response to different treatment regimens. The study indicates the involvement of TCSs in governing critical biological parameters of E. coli, ranging from regulating environmental stresses to intrinsic resistance. Managing E. coli's ability to utilize TCSs should prove a worthy consideration as therapeutic targets.

Recent work has revealed the constellations of genes involved in phenotypic resistance with several of these genes contributing to bacterial intrinsic resistance, and also encode key elements of bacterial physiological states (Fajardo et al. 2008; Tamae et al. 2008; Martinez and Rojo 2011). The current study of TCSs provides a link to the physiological state of bacteria and to the responses that microorganisms receive when confronted to different stresses including antibiotics. Thus, this knowledge will be relevant in providing a better understanding of molecular mechanisms of resistance and contribute towards new adjunctive alternatives to improve the efficacy of antibiotic therapy programs.

In conclusion, to combat AMR, we need to understand the evolutionary trajectories that underlie the acquisition of antibiotic resistance mutations and their 
pleiotropic aftermaths, which are the critical drivers of the evolution of AMR (Chapter 2), how bacterial fitness depend on the environment it lives on (Chapter 4) and, to counteract the problem we need novel methods, such as genetic screens that can identify targets that can restore the effectiveness of already existing antibiotics (Chapter 3). This research work will lead us to understand the evolution and genetic complexity of resistance and will be helpful in contributing to the knowledge of antibiotic resistance evolution in future studies. 


\section{Chapter 6}

\section{Future Perspectives}

The widespread use of antibiotics has increased the evolutionary selection pressure for the emergence of antimicrobial resistance (AMR). The projects presented in this thesis seek to expand our understanding of the genetic basis for the evolution of resistance, and also involve novel concepts to circumvent the resistance problem once it has arisen.

The knowledge to predict evolutionary trajectories plays an integral part in the drug-development program that can make better pre-informed decisions. This type of knowledge is crucial in evaluating the prospects of the emergence of resistance to novel drugs and managing AMR in the future. This includes the mutation supply rate, level of resistance, identification of resistance mutations, and their pleiotropic aftermaths (addressed in chapter 2 of this thesis). Currently there is limited quantitative data available that restricts efforts to predict the trajectory of resistance evolution, especially in relevant environments (clinical and natural). More insight is required to better predict the evolution of AMR. This useful knowledge comprises information about epistatic interactions and compensatory evolution, co-selection and horizontal gene transfer, and population bottlenecks and clonal interference. Thus, using additional risk-assessment steps, it may impart better predictions to determine the success of a drug candidate in clinical development. 
The limited supply of antimicrobials has prompted the search for alternative approaches that can potentiate the effects of existing antibiotics. Investigating novel drug targets that exist in bacterial pathogens through interactive chemical-genetic sensitivity screens has gained a lot of interest. In a high-throughput approach using small RNA (sRNA) (addressed in chapter 3 of this thesis), I identified Escherichia coli genes that could be targeted to reverse quinolone resistance. In the next steps, we require a competent delivery system for efficient expression of synthetic sRNA constructs. Phage therapy or conjugative plasmids might be used as a successful sRNA delivery system (Lu and Collins 2009; Rastogi et al. 2018).

Antibiotic tolerance plays a critical role in bacterial survival, which has largely been an underappreciated area. The metabolic state of a bacterium depends on the environment where it lives, which in turn impacts antibiotic tolerance. The tolerance conferring effects are induced by environmental stresses and genetic factors that are translated into physiological responses. This is mediated largely by two-component signal transduction systems (TCS) in bacteria (addressed in chapter 4 of this thesis), which mediate signaling through the envelope stress response. Further steps require an accurate understanding of the clinical manifestation of antibiotic tolerance and the contribution of TCSs. TCSs are one of the promising combination therapy approaches that can be explored further to gain high-quality drug targets for potentiation of known antibacterial drugs.

To control or reduce the AMR evolution we need further effective strategies to improve our antimicrobials. Our knowledge of various mechanisms involved in 
underpinning AMR has expanded in recent years. Researchers have also recognized challenges (Payne et al. 2007; Tommasi et al. 2015) that constrain efforts to restrict AMR. Some of the effective strategies include, (i) Potentiation approaches. Highthroughput random transposon mutagenesis libraries in Pseudomonas aeruginosa and E. coli led to the identification of many genes responsible for intrinsic resistance to different classes of antibiotics (Breidenstein et al. 2008; Fajardo et al. 2008; Tamae et al. 2008; Liu et al. 2010). This has led the way for potential drug combinations whereby one agent can inhibit intrinsic resistance mechanisms to enhance the activities of other antibiotics. For example, inactivation of non-essential $E$. coli genes, such as $f a b I, \operatorname{rec} Q, \operatorname{sap} C$, and $d a c A$ can greatly potentiate the activity of already existing drugs, including rifampicin, aminoglycosides, triclosan, and some B-lactams (Liu et al. 2010; Blake and Neill 2013; Cox et al. 2014). (ii) Active uptake approaches. By taking advantage of membrane transporters, antibiotic influx can be increased. For example, fosfomycin uses bacterial sugar transporters to enter the cells (Czaplewski et al. 2016). (iii) Cycling of antibiotics. We can prolong the life span of already existing antibiotics. For example, a fluoroquinolone plus a macrolide or a ß-lactam plus an aminoglycoside or tetracycline (with different modes of action). This type of approach has been used in the past for treatment of cancer infection, however a comprehensive pharmacodynamic matching is essential, such as standardized predetermined concentrations of antibiotics for the maximum synergy (Bergstrom et al. 2004; Masterton 2005; Davies and Davies 2010). Therefore, this type of system does not provide a long-term solution, but certainly reduces the selection pressures for resistance. (iv) Combinatorial approaches. Attempts have been made to inhibit or bypass resistance mechanisms in pathogens, especially with 
ß-lactam antibiotics. For example, Clavulanic acid or natural products, such as Aspergilliomarasmine A (AMA) inhibits ß-lactamases enzymes and is used in combination with ß-lactam antibiotics (Reading and Cole 1977; King et al. 2014). Also, collateral sensitivity or cross-resistance strategies are encouraging options in a combination therapy (Imamovic and Sommer 2013; Pál et al. 2015; Lázár et al. 2015). (v) Non-conventional approaches. There are some alternative approaches to address bacterial infections, such as phage therapy. For example, Sahota et al. 2015 used phages directly inhaled into the lungs against $P$. aeruginosa to treat cystic fibrosis. Also, Nale et al. 2015 used a phage mixture in the gastrointestinal tract for the treatment of Clostridium difficile infection. Restoring the patient's microbiome (usually in C. difficile infection) is another non-conventional approach. Gerding et al. 2015 used nontoxinogenic C. difficile that can outcompete the infecting toxic $C$. difficile, helping to re-establish the host microbiome. Monoclonal antibodies can also be used as an alternative to antibiotics. Antibodies have already been successfully used in inflammatory diseases treatment (Czaplewski et al. 2016; Pegu et al. 2017; Bagnoli and Payne 2017). DiGiandomenico et al. (2014) showed a bispecific monoclonal antibody to target virulence factor (PcrV) and persistence factor (PsI) in P. aeruginosa.

All of these mentioned innovative approaches face challenges to be successfully used, such as efficacy and robustness in clinical development path or cost of development. But, investing in these collaborative measures can significantly advance our ability to control AMR. In addition, these efforts will only be successful if taken as a comprehensible international effort (O'Neill 2014; Boeckel et al. 2014). In essence, for 
our future, we have to use the best of available technologies, knowledge, and expertise to circumvent the resistance crisis and develop next generation antimicrobials. 


\section{Literature cited}

Aiba, H., \& Mizuno, T. (1990). Phosphorylation of a bacterial activator protein, OmpR, by a protein kinase, EnvZ, stimulates the transcription of the $о т p F$ and $о т p C$ genes in Escherichia coli. FEBS Letters, 261(1), 19-22. https://doi.org/10.1016/0014-

$\underline{5793(90) 80626-\mathrm{T}}$

Alam, M. K., Alhhazmi, A., DeCoteau, J. F., Luo, Y., \& Geyer, C. R. (2016). RecA inhibitors potentiate antibiotic activity and block evolution of antibiotic resistance. Cell Chemical Biology, 23(3), 381-391. $\underline{\text { https://doi.org/10.1016/j.chembiol.2016.02.010 }}$

Alekshun, M. N., \& Levy, S. B. (1997). Regulation of chromosomally mediated multiple antibiotic resistance: The mar regulon. Antimicrobial Agents and Chemotherapy, 41(10), 2067-2075. https://doi.org/10.1128/AAC.41.10.2067

Alekshun, M. N., \& Levy, S. B. (1999a). Alteration of the repressor activity of MarR, the negative regulator of the Escherichia coli marRAB locus, by multiple chemicals in vitro. Journal of Bacteriology, 181(15), 4669-4672.

https://www.ncbi.nlm.nih.gov/pubmed/10419969 
Alekshun, M. N., \& Levy, S. B. (1999b). Characterization of MarR superrepressor mutants. Journal of Bacteriology, 181(10), 3303-3306.

\section{http://jb.asm.org/content/181/10/3303.abstract}

Alekshun, M. N., \& Levy, S. B. (2007). Molecular mechanisms of antibacterial multidrug resistance. Cell, 128(6), 1037-1050. https://doi.org/10.1016/j.cell.2007.03.004

Alekshun, M. N., Kim, Y. S., \& Levy, S. B. (2000). Mutational analysis of MarR, the negative regulator of marRAB expression in Escherichia coli, suggests the presence of two regions required for DNA binding. Molecular Microbiology, 35(6), 13941404. https://doi.org/10.1046/j.1365-2958.2000.01802.x

Alekshun, M. N., Levy, S. B., Mealy, T. R., Seaton, B. A., \& Head, J. F. (2001). The crystal structure of MarR, a regulator of multiple antibiotic resistance, at $2.3 \AA$ resolution. Nature Structural Biology, 8(8), 710-714. https://doi.org/10.1038/90429

Allison, J. L., Hartman, R. E., Hartman, R. S., Wolfe, A. D., Ciak, J., \& Hahn, F. E. (1962). Mode of action of chloramphenicol. VII. Growth and multiplication of Escherichia coli in the presence of chloramphenicol. Journal of Bacteriology, 83(3), 609-615. https://www.ncbi.nlm.nih.gov/pubmed/13860618

Allison, K. R., Brynildsen, M. P., \& Collins, J. J. (2011). Metabolite-enabled eradication of bacterial persisters by aminoglycosides. Nature, 473, 216-220. https://doi.org/10.1038/nature10069 
Anderson, G. J. (2004). Quinolone antimicrobial agents, 3rd Edition. Emerging Infectious Diseases, 10(6), 1177. https://doi.org/10.3201/eid1006.040025

Andersson, D. I. (2006). The biological cost of mutational antibiotic resistance: Any practical conclusions? Current Opinion in Microbiology, 9(5), 461-465. https://doi.org/10.1016/j.mib.2006.07.002

Andersson, D. I., \& Hughes, D. (2010). Antibiotic resistance and its cost: Is it possible to reverse resistance? Nature Reviews Microbiology, 8, 260-271. https://doi.org/10.1038/nrmicro2319

Andersson, D. I., \& Hughes, D. (2017). Selection and transmission of antibiotic-resistant bacteria. Microbiology Spectrum, 5(4), 117-137. http://dx.doi.org/10.1128/microbiolspec.MTBP-0013-2016

Andersson, D. I., \& Levin, B. R. (1999). The biological cost of antibiotic resistance. Current Opinion in Microbiology, 2(5), 489-493. https://doi.org/10.1016/S1369$\underline{5274(99) 00005-3}$

Andersson, M. I., \& MacGowan, A. P. (2003). Development of the quinolones. Journal of Antimicrobial Chemotherapy, 51(suppl_1), 1-11. https://doi.org/10.1093/jac/dkg212

Andrews, J. M. (2001). Determination of minimum inhibitory concentrations. Journal of Antimicrobial Chemotherapy, 48(suppl_1), 5-16. https://doi.org/10.1093/jac/48.suppl_1.5 
Appelbaum, P. C., \& Hunter, P. A. (2000). The fluoroquinolone antibacterials: Past, present and future perspectives. International Journal of Antimicrobial Agents, 16(1), 5-15. https://doi.org/10.1016/S0924-8579(00)00192-8

Argaman, L., Hershberg, R., Vogel, J., Bejerano, G., Wagner, E. G. H., Margalit, H., \& Altuvia, S. (2001). Novel small RNA-encoding genes in the intergenic regions of Escherichia coli. Current Biology, 11(12), 941-950. https://doi.org/10.1016/S0960$\underline{9822(01) 00270-6}$

Audrain, B., Ferrières, L., Zairi, A., Soubigou, G., Dobson, C., Coppée, J.-Y., ... Ghigo, J.-M. (2013). Induction of the Cpx envelope stress pathway contributes to Escherichia coli tolerance to antimicrobial peptides. Applied and Environmental Microbiology, 79(24), 7770-7779. https://doi.org/10.1128/AEM.02593-13

Austin, D. J., Kristinsson, K. G., \& Anderson, R. M. (1999). The relationship between the volume of antimicrobial consumption in human communities and the frequency of resistance. Proceedings of the National Academy of Sciences of the United States of America, 96(3), 1152-1156. https://doi.org/10.1073/pnas.96.3.1152

Azéma, J., Guidetti, B., Korolyov, A., Kiss, R., Roques, C., Constant, P., ... MaletMartino, M. (2011). Synthesis of lipophilic dimeric C-7/C-7-linked ciprofloxacin and C-6/C-6-linked levofloxacin derivatives. Versatile in vitro biological evaluations of monomeric and dimeric fluoroquinolone derivatives as potential antitumor, antibacterial or antimycobacterial agents. European Journal of 
Medicinal Chemistry, 46(12), 6025-6038.

https://doi.org/10.1016/j.ejmech.2011.10.014

Baba, T., Ara, T., Hasegawa, M., Takai, Y., Okumura, Y., Baba, M., ... Mori, H. (2006). Construction of Escherichia coli K-12 in-frame, single-gene knockout mutants: the Keio collection. Molecular Systems Biology, 2, 2006.0008. https://doi.org/10.1038/msb4100050

Bagel, S., Hüllen, V., Wiedemann, B., \& Heisig, P. (1999). Impact of gyrA and parC mutations on quinolone resistance, doubling time, and supercoiling degree of Escherichia coli. Antimicrobial Agents and Chemotherapy, 43(4), 868-875. http://doi.org/10.1128/AAC.43.4.868

Bagnoli, F., \& Payne, D. J. (2017). Reaction: Alternative modalities to address antibioticresistant pathogens. Chem, 3(3), 369-372. https://doi.org/10.1016/j.chempr.2017.08.017

Baker, M. D., Wolanin, P. M., \& Stock, J. B. (2006). Signal transduction in bacterial chemotaxis. BioEssays, 28(1), 9-22. https://doi.org/10.1002/bies.20343

Ball, P. (2000). Quinolone generations: Natural history or natural selection? Journal of Antimicrobial Chemotherapy, 46(suppl_3), 17-24. https://doi.org/10.1093/oxfordjournals.jac.a020889

Baquero, M. R., Bouzon, M., Varea, J., \& Moreno, F. (1995). sbmC, a stationary-phase induced SOS Escherichia coli gene, whose product protects cells from the DNA 
replication inhibitor microcin B17. Molecular Microbiology, 18(2), 301-311. https://doi.org/10.1111/j.1365-2958.1995.mmi_18020301.x

Baranova, N., \& Nikaido, H. (2002). The BaeSR two-component regulatory system activates transcription of the yegMNOB $(m d t A B C D)$ transporter gene cluster in Escherichia coli and increases its resistance to novobiocin and deoxycholate. Journal of Bacteriology, 184(15), 4168-4176. https://doi.org/10.1128/jb.184.15.4168-4176.2002

Barber, M., \& Rozwadowska-Dowzenko, M. (1948). Infection by penicillin-resistant Staphylococci. The Lancet, 252(6530), 641-644. https://doi.org/10.1016/S0140$\underline{6736(48) 92166-7}$

Barnard, F. M., \& Maxwell, A. (2001). Interaction between DNA gyrase and quinolones: Effects of alanine mutations at GyrA subunit residues Ser(83) and Asp(87). Antimicrobial Agents and Chemotherapy, 45(7), 1994-2000. $\underline{\text { https://doi.org/10.1128/AAC.45.7.1994-2000.2001 }}$

Barrett, J. F., \& Hoch, J. A. (1998). Two-component signal transduction as a target for microbial anti-infective therapy. Antimicrobial Agents and Chemotherapy, 42(7), 1529-1536. https://www.ncbi.nlm.nih.gov/pubmed/9660978

Barrett, J. F., Goldschmidt, R. M., Lawrence, L. E., Foleno, B., Chen, R., Demers, J. P., ... Hoch, J. A. (1998). Antibacterial agents that inhibit two-component signal transduction systems. Proceedings of the National Academy of Sciences of the 
United States of America, 95(9), 5317-5322.

https://doi.org/10.1073/pnas.95.9.5317

Bartlett, J. G., Gilbert, D. N., \& Spellberg, B. (2013). Seven Ways to Preserve the Miracle of Antibiotics. Clinical Infectious Diseases, 56(10), 1445-1450. https://doi.org/10.1093/cid/cit070

Basra, P., Alsaadi, A., Bernal-Astrain, G., O’Sullivan, M. L., Hazlett, B., Clarke, L. M., ... Wong, A. (2018). Fitness tradeoffs of antibiotic resistance in extraintestinal pathogenic Escherichia coli. Genome Biology and Evolution, 10(2), 667-679. https://doi.org/10.1093/gbe/evy030

Bassetti, M., Righi, E., \& Viscoli, C. (2008). Novel $\beta$-lactam antibiotics and inhibitor combinations. Expert Opinion on Investigational Drugs, 17(3), 285296. https://doi.org/10.1517/13543784.17.3.285

Batchelor, E., Walthers, D., Kenney, L. J., \& Goulian, M. (2005). The Escherichia coli CpxA-CpxR envelope stress response system regulates expression of the porins ompF and ompC. Journal of Bacteriology, 187(16), 5723-5731. https://doi.org/10.1128/JB.187.16.5723-5731.2005

Baucheron, S., Monchaux, I., Le Hello, S., Weill, F.-X., \& Cloeckaert, A. (2014). Lack of efflux mediated quinolone resistance in Salmonella enterica serovars Typhi and Paratyphi A. Frontiers in Microbiology, 5, 12.

https://doi.org/10.3389/fmicb.2014.00012 
Baudry-Simner, P. J., Singh, A., Karlowsky, J. A., Hoban, D. J., Zhanel, G. G., \& Alliance, C. A. R. (2012). Mechanisms of reduced susceptibility to ciprofloxacin in Escherichia coli isolates from Canadian hospitals. The Canadian Journal of Infectious Diseases \& Medical Microbiology, 23(3), e60-e64. https://doi.org/10.1155/2012/569093

Becnel Boyd, L., Maynard, M. J., Morgan-Linnell, S. K., Horton, L. B., Sucgang, R., Hamill, R. J., ... Zechiedrich, L. (2009). Relationships among ciprofloxacin, gatifloxacin, levofloxacin, and norfloxacin MICs for fluoroquinolone-resistant Escherichia coli clinical isolates. Antimicrobial Agents and Chemotherapy, 53(1), 229-234. https://doi.org/10.1128/AAC.00722-08

Behr, S., Fried, L., \& Jung, K. (2014). Identification of a novel nutrient-sensing histidine kinase/response regulator network in Escherichia coli. Journal of Bacteriology, 196(11), 2023-2029. https://doi.org/10.1128/JB.01554-14

Bem, A. E., Velikova, N., Pellicer, M. T., Baarlen, P. van, Marina, A., \& Wells, J. M. (2015). Bacterial histidine kinases as novel antibacterial drug targets. ACS Chemical Biology, 10(1), 213-224. https://doi.org/10.1021/cb5007135

Bergstrom, C. T., Lo, M., \& Lipsitch, M. (2004). Ecological theory suggests that antimicrobial cycling will not reduce antimicrobial resistance in hospitals. Proceedings of the National Academy of Sciences of the United States of America, 101(36), 13285 LP - 13290. https://doi.org/10.1073/pnas.0402298101 
Bernadac, A., Gavioli, M., Lazzaroni, J. C., Raina, S., \& Lloubès, R. (1998). Escherichia coli tol-pal mutants form outer membrane vesicles. Journal of Bacteriology, 180(18), 4872-4878. https://www.ncbi.nlm.nih.gov/pubmed/9733690

Betitra, Y., Teresa, V., Miguel, V., \& Abdelaziz, T. (2014). Determinants of quinolone resistance in Escherichia coli causing community-acquired urinary tract infection in Bejaia, Algeria. Asian Pacific Journal of Tropical Medicine, 7(6), 462-467. https://doi.org/10.1016/S1995-7645(14)60075-4

Bhatnagar, K., \& Wong, A. (2019). The mutational landscape of quinolone resistance in Escherichia coli. PLOS One (Under review).

Björkman, J., Hughes, D., \& Andersson, D. I. (1998). Virulence of antibiotic-resistant Salmonella typhimurium. Proceedings of the National Academy of Sciences of the United States of America, 95(7), 3949-3953.

https://doi.org/10.1073/pnas.95.7.3949

Björkman, J., Nagaev, I., Berg, O. G., Hughes, D., \& Andersson, D. I. (2000). Effects of environment on compensatory mutations to ameliorate costs of antibiotic resistance. Science, 287(5457), 1479-1482.

https://doi.org/10.1126/science.287.5457.1479

Björkman, J., Samuelsson, P., Andersson, D. I., \& Hughes, D. (1999). Novel ribosomal mutations affecting translational accuracy, antibiotic resistance and virulence of Salmonella typhimurium. Molecular Microbiology, 31(1), 53-58.

https://doi.org/10.1046/j.1365-2958.1999.01142.x 
Blair, J. M. A., Webber, M. A., Baylay, A. J., Ogbolu, D. O., \& Piddock, L. J. V. (2014). Molecular mechanisms of antibiotic resistance. Nature Reviews Microbiology, 13, 42-51. https://doi.org/10.1038/nrmicro3380

Blake, K. L., \& O’Neill, A. J. (2012). Transposon library screening for identification of genetic loci participating in intrinsic susceptibility and acquired resistance to antistaphylococcal agents. Journal of Antimicrobial Chemotherapy, 68(1), 12-16. https://doi.org/10.1093/jac/dks373

Boeckel, T. P. Van, Gandra, S., Ashok, A., Caudron, Q., Grenfell, B. T., Levin, S. A., \& Laxminarayan, R. (2014). Global antibiotic consumption 2000 to 2010: An analysis of national pharmaceutical sales data. The Lancet Infectious Diseases, 14(8), 742750. https://doi.org/10.1016/S1473-3099(14)70780-7

Bolger, A. M., Lohse, M., \& Usadel, B. (2014). Trimmomatic: a flexible trimmer for Illumina sequence data. Bioinformatics (Oxford, England), 30(15), 2114-2120. https://doi.org/10.1093/bioinformatics/btu170

Bolon, M. K. (2009). The newer fluoroquinolones. Infectious Disease Clinics of North America, 23(4), 1027-1051. https://doi.org/10.1016/j.idc.2009.06.003

Borrell, S., Teo, Y., Giardina, F., Streicher, E. M., Klopper, M., Feldmann, J., ... Gagneux, S. (2013). Epistasis between antibiotic resistance mutations drives the evolution of extensively drug-resistant tuberculosis. Evolution, Medicine, and Public Health, 2013(1), 65-74. https://doi.org/10.1093/emph/eot003 
Boudreau, M. A., Fishovitz, J., Llarrull, L. I., Xiao, Q., \& Mobashery, S. (2015).

Phosphorylation of BlaR1 in manifestation of antibiotic resistance in methicillinresistant Staphylococcus aureus and its abrogation by small molecules. ACS Infectious Diseases, 1(10), 454-459. https://doi.org/10.1021/acsinfecdis.5b00086

Brandis, G., \& Hughes, D. (2013). Genetic characterization of compensatory evolution in strains carrying rроB Ser531Leu, the rifampicin resistance mutation most frequently found in clinical isolates. Journal of Antimicrobial Chemotherapy, 68(11), 2493-2497. https://doi.org/10.1093/jac/dkt224

Brandis, G., Pietsch, F., Alemayehu, R., \& Hughes, D. (2014). Comprehensive phenotypic characterization of rifampicin resistance mutations in Salmonella provides insight into the evolution of resistance in Mycobacterium tuberculosis. Journal of Antimicrobial Chemotherapy, 70(3), 680-685. https://doi.org/10.1093/jac/dku434

Breidenstein, E. B. M., Khaira, B. K., Wiegand, I., Overhage, J., \& Hancock, R. E. W. (2008). Complex ciprofloxacin resistome revealed by screening a Pseudomonas aeruginosa mutant library for altered susceptibility. Antimicrobial Agents and Chemotherapy, 52(12), 4486-4491. https://doi.org/10.1128/AAC.00222-08

Breland, E. J., Zhang, E. W., Bermudez, T., Martinez, C. R., \& Hadjifrangiskou, M. (2017). The Histidine Residue of QseC Is Required for Canonical Signaling between QseB and PmrB in Uropathogenic Escherichia coli. Journal of Bacteriology, 199(18), e00060-17. https://doi.org/10.1128/JB.00060-17 
Brino, L., Urzhumtsev, A., Mousli, M., Bronner, C., Mitschler, A., Oudet, P., \& Moras, D. (2000). Dimerization of Escherichia coli DNA-gyrase B provides a structural mechanism for activating the ATPase catalytic center. Journal of Biological Chemistry, 275(13), 9468-9475. http://doi.org/10.1074/jbc.275.13.9468

Brown, D. (2015). Antibiotic resistance breakers: Can repurposed drugs fill the antibiotic discovery void? Nature Reviews Drug Discovery, 14, 821-832. https://doi.org/10.1038/nrd4675

Bury-Moné, S., Nomane, Y., Reymond, N., Barbet, R., Jacquet, E., Imbeaud, S., ... Bouloc, P. (2009). Global analysis of extracytoplasmic stress signaling in Escherichia coli. PLOS Genetics, 5(9), e1000651. https://doi.org/10.1371/journal.pgen.1000651

Cai, S. J., \& Inouye, M. (2002). EnvZ-OmpR interaction and osmoregulation in Escherichia coli. Journal of Biological Chemistry, 277(27), 24155-24161. http://doi.org/10.1074/jbc.M110715200

Campbell, E. A., Korzheva, N., Mustaev, A., Murakami, K., Nair, S., Goldfarb, A., \& Darst, S. A. (2001). Structural mechanism for rifampicin inhibition of bacterial RNA polymerase. Cell, 104(6), 901-912. https://doi.org/10.1016/S0092-

\section{$\underline{8674(01) 00286-0}$}

Canadian antimicrobial resistance alliance (CARA). (2015). CANWARD susceptibility report. www.can-r.com 
Capra, E. J., \& Laub, M. T. (2012). Evolution of two-component signal transduction systems. Annual Review of Microbiology, 66, 325-347. https://dx.doi.org/10.1146\%2Fannurev-micro-092611-150039

Champoux, J. J. (2001). DNA Topoisomerases: Structure, function, and mechanism. Annual Review of Biochemistry, 70(1), 369-413. https://doi.org/10.1146/annurev.biochem.70.1.369

Chávez-Jacobo, V. M., Hernández-Ramírez, K. C., Romo-Rodríguez, P., Pérez-Gallardo, R. V., Campos-García, J., Gutiérrez-Corona, J. F., ... Ramírez-Díaz, M. I. (2018). CrpP Is a novel ciprofloxacin-modifying enzyme encoded by the Pseudomonas aeruginosa pUM505 plasmid. Antimicrobial Agents and Chemotherapy, 62(6), e02629-17. https://doi.org/10.1128/AAC.02629-17

Cheng, H. Y., Chen, Y. F., \& Peng, H. L. (2010). Molecular characterization of the PhoPQ-PmrD-PmrAB mediated pathway regulating polymyxin B resistance in Klebsiella pneumoniae CG43. Journal of Biomedical Science, 17(1), 60. $\underline{\text { https://doi.org/10.1186/1423-0127-17-60 }}$

Cheng, Z. F., \& Deutscher, M. P. (2002). Purification and characterization of the Escherichia coli exoribonuclease RNase R: Comparison with RNase II. Journal of Biological Chemistry, 277(24), 21624-21629. https://doi.org/10.1074/jbc.M202942200

Chopra, I., \& Roberts, M. (2001). Tetracycline antibiotics: Mode of action, applications, molecular biology, and epidemiology of bacterial resistance. Microbiology and 
Molecular Biology Reviews: MMBR, 65(2), 232-260.

https://doi.org/10.1128/MMBR.65.2.232-260.2001

Cingolani, P., Platts, A., Wang, L. L., Coon, M., Nguyen, T., Wang, L., ... Ruden, D. M. (2012). A program for annotating and predicting the effects of single nucleotide polymorphisms, SnpEff: SNPs in the genome of Drosophila melanogaster strain w1118; iso-2; iso-3. Fly, 6(2), 80-92. https://doi.org/10.4161/fly.19695

Cohen, S. P., Hächler, H., \& Levy, S. B. (1993). Genetic and functional analysis of the multiple antibiotic resistance (mar) locus in Escherichia coli. Journal of Bacteriology, 175(5), 1484-1492. https://doi.org/10.1128/jb.175.5.1484-1492.1993

Cohen, S. P., McMurry, L. M., Hooper, D. C., Wolfson, J. S., \& Levy, S. B. (1989). Cross-resistance to fluoroquinolones in multiple-antibiotic-resistant (Mar) Escherichia coli selected by tetracycline or chloramphenicol: Decreased drug accumulation associated with membrane changes in addition to OmpF reduction. Antimicrobial Agents and Chemotherapy, 33(8), 1318-1325. https://doi.org/10.1128/aac.33.8.1318

Cohen, T., Sommers, B., \& Murray, M. (2003). The effect of drug resistance on the fitness of Mycobacterium tuberculosis. The Lancet Infectious Diseases, 3(1), 1321. https://doi.org/10.1016/S1473-3099(03)00483-3

Collin, F., Karkare, S., \& Maxwell, A. (2011). Exploiting bacterial DNA gyrase as a drug target: Current state and perspectives. Applied Microbiology and Biotechnology, 92(3), 479-497. https://doi.org/10.1007/s00253-011-3557-z 
Comas, I., Borrell, S., Roetzer, A., Rose, G., Malla, B., Kato-Maeda, M., ... Gagneux, S. (2011). Whole-genome sequencing of rifampicin-resistant Mycobacterium tuberculosis strains identifies compensatory mutations in RNA polymerase genes. Nature Genetics, 44(1), 106-110. https://doi.org/10.1038/ng.1038

Conde, M. B., \& Lapa E Silva, J. R. (2011). New regimens for reducing the duration of the treatment of drug-susceptible pulmonary tuberculosis. Drug Development Research, 72(6), 501-508. https://doi.org/10.1002/ddr.20456

Cordell, H. J. (2002). Epistasis: What it means, what it doesn't mean, and statistical methods to detect it in humans. Human Molecular Genetics, 11(20), 2463-2468. https://doi.org/10.1093/hmg/11.20.2463

Corona, F., \& Martinez, J.L. (2013). Phenotypic resistance to antibiotics. Antibiotics, 2, 237-255. https://doi.org/10.3390/antibiotics2020237

Cox, G., \& Wright, G. D. (2013). Intrinsic antibiotic resistance: Mechanisms, origins, challenges and solutions. International Journal of Medical Microbiology, 303(6), 287-292. https://doi.org/10.1016/j.ijmm.2013.02.009

Cox, G., Koteva, K., \& Wright, G. D. (2014). An unusual class of anthracyclines potentiate Gram-positive antibiotics in intrinsically resistant Gram-negative bacteria. The Journal of Antimicrobial Chemotherapy, 69(7), 1844-1855. https://doi.org/10.1093/jac/dku057 
Cox, G., Sieron, A., King, A. M., Pascale, G. De, Pawlowski, A. C., Koteva, K., \& Wright, G. D. (2017). A common platform for antibiotic dereplication and adjuvant discovery. Cell Chemical Biology, 24(1), 98-109.

https://doi.org/10.1016/j.chembiol.2016.11.011

Cramariuc, O., Rog, T., Javanainen, M., Monticelli, L., Polishchuk, A. V, \& Vattulainen, I. (2012). Mechanism for translocation of fluoroquinolones across lipid membranes. Biochimica et Biophysica Acta (BBA) - Biomembranes, 1818(11), 2563-2571. https://doi.org/10.1016/j.bbamem.2012.05.027

Crofts, T. S., Gasparrini, A. J., \& Dantas, G. (2017). Next-generation approaches to understand and combat the antibiotic resistome. Nature Reviews. Microbiology, 15(7), 422-434. https://doi.org/10.1038/nrmicro.2017.28

Czaplewski, L., Bax, R., Clokie, M., Dawson, M., Fairhead, H., Fischetti, V. A., ... Rex, J. H. (2016). Alternatives to antibiotics - a pipeline portfolio review. The Lancet Infectious Diseases, 16(2), 239-251. https://doi.org/10.1016/S1473$\underline{3099(15) 00466-1}$

D’Costa, V. M., McGrann, K. M., Hughes, D. W., \& Wright, G. D. (2006). Sampling the antibiotic resistome. Science, 311(5759), 374-377.

https://doi.org/10.1126/science. 1120800

Dalebroux, Z. D., \& Miller, S. I. (2014). Salmonellae PhoPQ regulation of the outer membrane to resist innate immunity. Current Opinion in Microbiology, 17, 106113. https://doi.org/10.1016/j.mib.2013.12.005 
Dam, S., Pagès, J.-M., \& Masi, M. (2018). Stress responses, outer membrane permeability control and antimicrobial resistance in Enterobacteriaceae. Microbiology, 164(3), 260-267. https://dx.doi.org/10.1099/mic.0.000613

Davies, J., \& Davies, D. (2010). Origins and evolution of antibiotic resistance. Microbiology and Molecular Biology Reviews, 74(3), 417-433. https://doi.org/10.1128/MMBR.00016-10

Debabov, D. (2013). Antibiotic resistance: Origins, mechanisms, approaches to counter. Applied Biochemistry and Microbiology, 49(8), 665-671. https://doi.org/10.1134/S0003683813080024

Deguchi, T., Yasuda, M., Nakano, M., Ozeki, S., Kanematsu, E., Nishino, Y., ... Kawada, Y. (1997). Detection of mutations in the gyrA and $\operatorname{parC}$ genes in quinolone-resistant clinical isolates of Enterobacter cloacae. Journal of Antimicrobial Chemotherapy, 40(4), 543-549. https://doi.org/10.1093/jac/40.4.543

Dehio, C., \& Meyer, M. (1997). Maintenance of broad-host-range incompatibility group $\mathrm{P}$ and group Q plasmids and transposition of $\mathrm{Tn} 5$ in Bartonella henselae following conjugal plasmid transfer from Escherichia coli. Journal of Bacteriology, 179(2), 538-540. https://doi.org/10.1128/jb.179.2.538-540.1997

Delhaye, A., Collet, J.-F., \& Laloux, G. (2016). Fine-Tuning of the Cpx Envelope Stress Response Is Required for Cell Wall Homeostasis in Escherichia coli. MBio, 7(1), e00047-16. https://doi.org/10.1128/mBio.00047-16 
Deutch, A. H., Smith, C. J., Rushlow, K. E., \& Kretschmer, P. J. (1982). Escherichia coli delta 1-pyrroline-5-carboxylate reductase: Gene sequence, protein overproduction and purification. Nucleic Acids Research, 10(23), 7701-7714.

https://doi.org/10.1093/nar/10.23.7701

DiGate, R. J., \& Marians, K. J. (1988). Identification of a potent decatenating enzyme from Escherichia coli. Journal of Biological Chemistry, 263(26), 13366-13373. http://www.jbc.org/content/263/26/13366.abstract

DiGiandomenico, A., Keller, A. E., Gao, C., Rainey, G. J., Warrener, P., Camara, M. M., ... Stover, C. K. (2014). A multifunctional bispecific antibody protects against Pseudomonas aeruginosa. Science Translational Medicine, 6(262), 262ra155 LP262ra155. https://doi.org/10.1126/scitranslmed.3009655

Domagala, J. M. (1994). Structure-activity and structure-side-effect relationships for the quinolone antibacterials. Journal of Antimicrobial Chemotherapy, 33(4), 685-706. https://doi.org/10.1093/jac/33.4.685

Donahue, J. P., \& Turnbough, C. L. (1994). Nucleotide-specific transcriptional pausing in the pyrBI leader region of Escherichia coli K-12. Journal of Biological Chemistry, 269(27), 18185-18191. http://www.jbc.org/content/269/27/18185.abstract

Dong, Y., Zhao, X., Domagala, J., \& Drlica, K. (1999). Effect of fluoroquinolone concentration on selection of resistant mutants of Mycobacterium bovis BCG and Staphylococcus aureus. Antimicrobial Agents and Chemotherapy, 43(7), 17561758. https://doi.org/10.1128/AAC.43.7.1756 
Dorel, C., Lejeune, P., \& Rodrigue, A. (2006). The Cpx system of Escherichia coli, a strategic signaling pathway for confronting adverse conditions and for settling biofilm communities? Research in Microbiology, 157(4), 306-314.

https://doi.org/10.1016/j.resmic.2005.12.003

Dörr, T., Lewis, K., \& Vulić, M. (2009). SOS response induces persistence to fluoroquinolones in Escherichia coli. PLOS Genetics, 5(12), e1000760. https://doi.org/10.1371/journal.pgen.1000760

Doumith, M., Ellington, M. J., Livermore, D. M., \& Woodford, N. (2009). Molecular mechanisms disrupting porin expression in ertapenem-resistant Klebsiella and Enterobacter spp. clinical isolates from the UK. Journal of Antimicrobial Chemotherapy, 63(4), 659-667. https://doi.org/10.1093/jac/dkp029

Drawz, S. M., \& Bonomo, R. A. (2010). Three decades of beta-lactamase inhibitors. Clinical Microbiology Reviews, 23(1), 160-201. https://doi.org/10.1128/CMR.00037-09

Drawz, S. M., Papp-Wallace, K. M., \& Bonomo, R. A. (2014). New $\beta$-lactamase inhibitors: A therapeutic renaissance in an MDR world. Antimicrobial Agents and Chemotherapy, 58(4), 1835-1846. https://doi.org/10.1128/AAC.00826-13

Drlica, K., \& Zhao, X. (1997). DNA gyrase, topoisomerase IV, and the 4-quinolones. Microbiology and Molecular Biology Reviews : MMBR, 61(3), 377-392. https://www.ncbi.nlm.nih.gov/pubmed/9293187 
Drlica, K., Malik, M., Kerns, R. J., \& Zhao, X. (2008). Quinolone-mediated bacterial death. Antimicrobial Agents and Chemotherapy, 52(2), 385-392. https://doi.org/10.1128/AAC.01617-06

Du Toit, A. (2017). Efflux pumps, fitness and virulence. Nature Reviews Microbiology, 15, 512-513. https://doi.org/10.1038/nrmicro.2017.97

Durão, P., Trindade, S., Sousa, A., \& Gordo, I. (2015). Multiple Resistance at No Cost: Rifampicin and Streptomycin a dangerous liaison in the spread of antibiotic resistance. Molecular Biology and Evolution, 32(10), 2675-2680. https://doi.org/10.1093/molbev/msv143

Echols, H., Lu, C., \& Burgers, P. M. (1983). Mutator strains of Escherichia coli, mutD and $d n a Q$, with defective exonucleolytic editing by DNA polymerase III holoenzyme. Proceedings of the National Academy of Sciences of the United States of America, 80(8), 2189-2192. https://doi.org/10.1073/pnas.80.8.2189

Eggenhofer, F., Tafer, H., Stadler, P. F., \& Hofacker, I. L. (2011). RNApredator: Fast accessibility-based prediction of sRNA targets. Nucleic Acids Research, 39(Web Server issue), W149-W154. https://doi.org/10.1093/nar/gkr467

Eguchi, Y., Itou, J., Yamane, M., Demizu, R., Yamato, F., Okada, A., ... Utsumi, R. (2007). B1500, a small membrane protein, connects the two-component systems EvgS/EvgA and PhoQ/PhoP in Escherichia coli. Proceedings of the National Academy of Sciences of the United States of America, 104(47), 18712-18717. https://doi.org/10.1073/pnas.0705768104 
Ellis, H. M., Yu, D., DiTizio, T., \& Court, D. L. (2001). High efficiency mutagenesis, repair, and engineering of chromosomal DNA using single-stranded oligonucleotides. Proceedings of the National Academy of Sciences of the United States of America, 98(12), 6742-6746. https://doi.org/10.1073/pnas.121164898

Emmerson, A. M., \& Jones, A. M. (2003). The quinolones: Decades of development and use. Journal of Antimicrobial Chemotherapy, 51(suppl_1), 13-20. https://doi.org/10.1093/jac/dkg208

Enne, V. I. (2010). Reducing antimicrobial resistance in the community by restricting prescribing: Can it be done? Journal of Antimicrobial Chemotherapy, 65(2), 179182. https://doi.org/10.1093/jac/dkp443

Enne, V. I., Livermore, D. M., Stephens, P., \& Hall, L. M. C. (2001). Persistence of sulphonamide resistance in Escherichia coli in the UK despite national prescribing restriction. The Lancet, 357(9265), 1325-1328. https://doi.org/10.1016/S0140-

\section{$\underline{6736(00) 04519-0}$}

Everett, M. J., Jin, Y. F., Ricci, V., \& Piddock, L. J. (1996). Contributions of individual mechanisms to fluoroquinolone resistance in 36 Escherichia coli strains isolated from humans and animals. Antimicrobial Agents and Chemotherapy, 40(10), 23802386. https://doi.org/10.1128/AAC.40.10.2380

Fair, R. J., \& Tor, Y. (2014). Antibiotics and bacterial resistance in the 21 st century. Perspectives in Medicinal Chemistry, 6, 25-64. https://doi.org/10.4137/PMC.S14459 
Fajardo, A., \& Martínez, J. L. (2008). Antibiotics as signals that trigger specific bacterial responses. Current Opinion in Microbiology, 11(2), 161-167. https://doi.org/10.1016/j.mib.2008.02.006

Fajardo, A., Martínez-Martín, N., Mercadillo, M., Galán, J. C., Ghysels, B., Matthijs, S., ... Martínez, J. L. (2008). The neglected intrinsic resistome of bacterial pathogens. PLOS One, 3(2), e1619-e1619. https://doi.org/10.1371/journal.pone.0001619

Farha, M. A., \& Brown, E. D. (2013). Discovery of antibiotic adjuvants. Nature Biotechnology, 31, 120-122. https://doi.org/10.1038/nbt.2500

Fernández, L., \& Hancock, R. E. W. (2012). Adaptive and mutational resistance: Role of porins and efflux pumps in drug resistance. Clinical Microbiology Reviews, 25(4), 661-681. https://doi.org/10.1128/CMR.00043-12

Figge, R. M., Ramseier, T. M., \& Saier Jr, M. H. (1994). The mannitol repressor (MtlR) of Escherichia coli. Journal of Bacteriology, 176(3), 840-847. https://doi.org/10.1128/jb.176.3.840-847.1994

Finn, R. D., Coggill, P., Eberhardt, R. Y., Eddy, S. R., Mistry, J., Mitchell, A. L., ... Bateman, A. (2016). The Pfam protein families database: Towards a more sustainable future. Nucleic Acids Research, 44(D1), D279-D285. https://doi.org/10.1093/nar/gkv1344

Fischbach, M. A., \& Walsh, C. T. (2009). Antibiotics for emerging pathogens. Science, 325(5944), 1089-1093. https://doi.org/10.1126/science.1176667 
Forst, S. A., \& Roberts, D. L. (1994). Signal transduction by the EnvZ-OmpR phosphotransfer system in bacteria. Research in Microbiology, 145(5), 363-373. https://doi.org/10.1016/0923-2508(94)90083-3

Foster, P. L. (2006). Methods for determining spontaneous mutation rates. Methods in Enzymology, 409, 195-213. https://doi.org/10.1016/S0076-6879(05)09012-9

Fraud, S., \& Poole, K. (2011). Oxidative stress induction of the MexXY multidrug efflux genes and promotion of aminoglycoside resistance development in Pseudomonas aeruginosa. Antimicrobial Agents and Chemotherapy, 55(3), 1068-1074. https://doi.org/10.1128/AAC.01495-10

Fried, L., Behr, S., \& Jung, K. (2013). Identification of a target gene and activating stimulus for the $\mathrm{YpdA} / \mathrm{YpdB}$ histidine kinase/response regulator system in Escherichia coli. Journal of Bacteriology, 195(4), 807-815. https://doi.org/10.1128/JB.02051-12

Friedman, S. M., Lu, T., \& Drlica, K. (2001). Mutation in the DNA gyrase a gene of Escherichia coli that expands the quinolone resistance-determining region. Antimicrobial Agents and Chemotherapy, 45(8), 2378-2380. https://doi.org/10.1128/AAC.45.8.2378-2380.2001

Fukuda, H., \& Hiramatsu, K. (1999). Primary targets of fluoroquinolones in Streptococcus pneumoniae. Antimicrobial Agents and Chemotherapy, 43(2), 410412. http://doi.org/10.1128/AAC.43.2.410 
Gagneux, S., Long, C. D., Small, P. M., Van, T., Schoolnik, G. K., \& Bohannan, B. J. M. (2006). The competitive cost of antibiotic resistance in Mycobacterium tuberculosis. Science, 312(5782), 1944-1946.

$\underline{\text { https://doi.org/10.1126/science. } 1124410}$

Gao, R., \& Stock, A. M. (2009). Biological insights from structures of two-component proteins. Annual Review of Microbiology, 63, 133-154. https://doi.org/10.1146/annurev.micro.091208.073214

García-Dorado, A., \& Gallego, A. (2003). Comparing analysis methods for mutationaccumulation data: A simulation study. Genetics, 164(2), 807-819.

https://www.ncbi.nlm.nih.gov/pubmed/12807799

Garibyan, L., Huang, T., Kim, M., Wolff, E., Nguyen, A., Nguyen, T., ... Miller, J. H. (2003). Use of the $r p o B$ gene to determine the specificity of base substitution mutations on the Escherichia coli chromosome. DNA Repair, 2(5), 593-608. https://doi.org/10.1016/S1568-7864(03)00024-7

Gerding, D. N., Meyer, T., Lee, C., Cohen, S. H., Murthy, U. K., Poirier, A., ... Villano, S. (2015). Administration of spores of nontoxigenic Clostridium difficile strain M3 for prevention of recurrent $C$. difficile infection: A randomized clinical trial. JAMA, 313(17), 1719-1727. https://doi.org/10.1001/jama.2015.3725

Gerrish, P.J. \& Lenski, R.E. (1998). The fate of competing beneficial mutations in an asexual population. Genetica, 102, 127-144.

https://doi.org/10.1023/A:1017067816551 
Gill, E. E., Franco, O. L., \& Hancock, R. E. W. (2015). Antibiotic adjuvants: Diverse strategies for controlling drug-resistant pathogens. Chemical Biology \& Drug Design, 85(1), 56-78. https://doi.org/10.1111/cbdd.12478

Girgis, H. S., Hottes, A. K., \& Tavazoie, S. (2009). Genetic architecture of intrinsic antibiotic susceptibility. PlOS One, 4(5), e5629-e5629. https://doi.org/10.1371/journal.pone.0005629

Gomez, M. J., \& Neyfakh, A. A. (2006). Genes involved in intrinsic antibiotic resistance of Acinetobacter baylyi. Antimicrobial Agents and Chemotherapy, 50(11), 35623567. https://doi.org/10.1128/AAC.00579-06

González-Bello, C. (2017). Antibiotic adjuvants - A strategy to unlock bacterial resistance to antibiotics. Bioorganic \& Medicinal Chemistry Letters, 27(18), 42214228. https://doi.org/10.1016/j.bmcl.2017.08.027

Gooderham, W. J., \& Hancock, R. E. W. (2009). Regulation of virulence and antibiotic resistance by two-component regulatory systems in Pseudomonas aeruginosa. FEMS Microbiology Reviews, 33(2), 279-294. https://doi.org/10.1111/j.1574$\underline{6976.2008 .00135 . x}$

Gotoh, Y., Eguchi, Y., Watanabe, T., Okamoto, S., Doi, A., \& Utsumi, R. (2010). Twocomponent signal transduction as potential drug targets in pathogenic bacteria. Current Opinion in Microbiology, 13(2), 232-239. https://doi.org/10.1016/j.mib.2010.01.008 
Gottesman, B. S., Carmeli, Y., Shitrit, P., \& Chowers, M. (2009). Impact of quinolone restriction on resistance patterns of Escherichia coli isolated from urine by culture in a community setting. Clinical Infectious Diseases, 49(6), 869-875. https://doi.org/10.1086/605530

Gottesman, S. (2004). The small RNA regulators of Escherichia coli: Roles and Mechanisms. Annual Review of Microbiology, 58(1), 303-328. https://doi.org/10.1146/annurev.micro.58.030603.123841

Gould, I. M., \& Bal, A. M. (2013). New antibiotic agents in the pipeline and how they can help overcome microbial resistance. Virulence, 4(2), 185-191. https://doi.org/10.4161/viru.22507

Groisman, E. A. (2001). The pleiotropic two-component regulatory system PhoP-PhoQ. Journal of Bacteriology, 183(6), 1835-1842. https://doi.org/10.1128/JB.183.6.1835-1842.2001

Gross, M. (2013). Antibiotics in crisis. Current Biology, 23(24), R1063-R1065. https://doi.org/10.1016/j.cub.2013.11.057

Gross, R., Aricò, B., \& Rappuoli, R. (1989). Families of bacterial signal-transducing proteins. Molecular Microbiology, 3(11), 1661-1667. https://doi.org/10.1111/j.1365-2958.1989.tb00152.x

Guan, X., Xue, X., Liu, Y., Wang, J., Wang, Y., Wang, J., .. Pan, L. (2013). Plasmidmediated quinolone resistance - current knowledge and future perspectives. 
Journal of International Medical Research, 41(1), 20-30.

https://doi.org/10.1177/0300060513475965

Gubaev, A., \& Klostermeier, D. (2014). The mechanism of negative DNA supercoiling: A cascade of DNA-induced conformational changes prepares gyrase for strand passage. DNA Repair, 16, 23-34. https://doi.org/10.1016/j.dnarep.2014.01.011

Guckes, K. R., Breland, E. J., Zhang, E. W., Hanks, S. C., Gill, N. K., Algood, H. M. S., ... Hadjifrangiskou, M. (2017). Signaling by two-component system noncognate partners promotes intrinsic tolerance to polymyxin B in uropathogenic Escherichia coli. Science Signaling, 10(461), eaag1775.

https://doi.org/10.1126/scisignal.aag1775

Guckes, K. R., Kostakioti, M., Breland, E. J., Gu, A. P., Shaffer, C. L., Martinez, C. R., ... Hadjifrangiskou, M. (2013). Strong cross-system interactions drive the activation of the QseB response regulator in the absence of its cognate sensor. Proceedings of the National Academy of Sciences, 110(41), 16592-16597. $\underline{\text { https://doi.org/10.1073/pnas.1315320110 }}$

Guerrero, P., Collao, B., Álvarez, R., Salinas, H., Morales, E. H., Calderón, I. L., ... Gil, F. (2013). Salmonella enterica serovar Typhimurium BaeSR two-component system positively regulates $\operatorname{sodA}$ in response to ciprofloxacin. Microbiology (Reading, England), 159(Pt 10), 2049-2057. https://doi.org/10.1099/mic.0.066787- 
Gwynn, M. N., Portnoy, A., Rittenhouse, S. F., \& Payne, D. J. (2010). Challenges of antibacterial discovery revisited. Annals of the New York Academy of Sciences, 1213(1), 5-19. https://doi.org/10.1111/j.1749-6632.2010.05828.x

Hagiwara, D., Yamashino, T., \& Mizuno, T. (2004). A genome-wide view of the Escherichia coli BasS-BasR two-component system implicated in iron-responses. Bioscience, Biotechnology, and Biochemistry, 68(8), 1758-1767. https://doi.org/10.1271/bbb.68.1758

Hale, C. A., \& de Boer, P. A. J. (2002). ZipA is required for recruitment of FtsK, FtsQ, FtsL, and FtsN to the septal ring in Escherichia coli. Journal of Bacteriology, 184(9), 2552-2556. https://doi.org/10.1128/jb.184.9.2552-2556.2002

Hall, A. R., \& MacLean, R. C. (2016). Epistasis buffers the fitness effects of rifampicinresistance mutations in Pseudomonas aeruginosa. Evolution, 70(5), 1161. https://doi.org/10.1111/evo.12918

Hall, A. R., Iles, J. C., \& MacLean, R. C. (2011). The fitness cost of rifampicin resistance in Pseudomonas aeruginosa depends on demand for RNA polymerase. Genetics, 187(3), 817-822. https://doi.org/10.1534/genetics.110.124628

Hall, B. G., Acar, H., Nandipati, A., \& Barlow, M. (2013). Growth rates made easy. Molecular Biology and Evolution, 31(1), 232-238. https://doi.org/10.1093/molbev/mst187 
Hall, B. M., Ma, C.-X., Liang, P., \& Singh, K. K. (2009). Fluctuation analysis

CalculatOR: A web tool for the determination of mutation rate using LuriaDelbruck fluctuation analysis. Bioinformatics (Oxford, England), 25(12), 15641565. https://doi.org/10.1093/bioinformatics/btp253

Hallett, P., \& Maxwell, A. (1991). Novel quinolone resistance mutations of the Escherichia coli DNA gyrase A protein: Enzymatic analysis of the mutant proteins. Antimicrobial Agents and Chemotherapy, 35(2), 335-340. $\underline{\text { https://doi.org/10.1128/aac.35.2.335 }}$

Han, E. S., Cooper, D. L., Persky, N. S., Sutera Jr, V. A., Whitaker, R. D., Montello, M. L., \& Lovett, S. T. (2006). RecJ exonuclease: Substrates, products and interaction with SSB. Nucleic Acids Research, 34(4), 1084-1091.

https://doi.org/10.1093/nar/gkj503

Hancock, R. E. W., \& Sahl, H.-G. (2006). Antimicrobial and host-defense peptides as new anti-infective therapeutic strategies. Nature Biotechnology, 24(12), 15511557. https://doi.org/10.1038/nbt1267

Handel, A., Regoes, R. R., \& Antia, R. (2006). The role of compensatory mutations in the emergence of drug resistance. PLOS Computational Biology, 2(10), e137-e137. https://doi.org/10.1371/journal.pcbi.0020137

Harris, T. L., Worthington, R. J., Hittle, L. E., Zurawski, D. V, Ernst, R. K., \& Melander, C. (2014). Small molecule downregulation of PmrAB reverses lipid A modification 
and breaks colistin resistance. ACS Chemical Biology, 9(1), 122-127. https://doi.org/10.1021/cb400490k

Hawser, S., Lociuro, S., \& Islam, K. (2006). Dihydrofolate reductase inhibitors as antibacterial agents. Biochemical Pharmacology, 71(7), 941-948. $\underline{\text { https://doi.org/10.1016/j.bcp.2005.10.052 }}$

Hayes, M. V, \& Orr, D. C. (1983). Mode of action of ceftazidime: Affinity for the penicillin-binding proteins of Escherichia coli K12, Pseudomonas aeruginosa and Staphylococcus aureus. Journal of Antimicrobial Chemotherapy, 12(2), 119-126. https://doi.org/10.1093/jac/12.2.119

Heddle, J., \& Maxwell, A. (2002). Quinolone-binding pocket of DNA gyrase: Role of GyrB. Antimicrobial Agents and Chemotherapy, 46(6), 1805-1815. https://doi.org/10.1128/aac.46.6.1805-1815.2002

Heisig, P. (1996). Genetic evidence for a role of parC mutations in development of highlevel fluoroquinolone resistance in Escherichia coli. Antimicrobial Agents and Chemotherapy, 40(4), 879-885. https://doi.org/10.1128/AAC.40.4.879

Heisig, P., \& Tschorny, R. (1994). Characterization of fluoroquinolone-resistant mutants of Escherichia coli selected in vitro. Antimicrobial Agents and Chemotherapy, 38(6), 1284-1291. https://doi.org/10.1128/aac.38.6.1284 
Heller, R. C., \& Marians, K. J. (2005). The disposition of nascent strands at stalled replication forks dictates the pathway of replisome loading during restart. Molecular Cell, 17(5), 733-743. https://doi.org/10.1016/j.molcel.2005.01.019

Herring, C. D., Glasner, J. D., \& Blattner, F. R. (2003). Gene replacement without selection: regulated suppression of amber mutations in Escherichia coli. Gene, 311, 153-163. https://doi.org/10.1016/S0378-1119(03)00585-7

Hiasa, H., Yousef, D. O., \& Marians, K. J. (1996). DNA strand cleavage is required for replication fork arrest by a frozen topoisomerase-quinolone-DNA ternary complex. Journal of Biological Chemistry, 271(42), 26424-26429. $\underline{\text { http://doi.org/10.1074/jbc.271.42.26424 }}$

Hidese, R., Mihara, H., Kurihara, T., \& Esaki, N. (2014). Global identification of genes affecting iron-sulfur cluster biogenesis and iron homeostasis. Journal of Bacteriology, 196(6), 1238-1249. https://doi.org/10.1128/JB.01160-13

Hill, T. M., Tecklenburg, M. L., Pelletier, A. J., \& Kuempel, P. L. (1989). tus, the transacting gene required for termination of DNA replication in Escherichia coli, encodes a DNA-binding protein. Proceedings of the National Academy of Sciences of the United States of America, 86(5), 1593-1597. https://doi.org/10.1073/pnas.86.5.1593

Hirakawa, H., Nishino, K., Yamada, J., Hirata, T., \& Yamaguchi, A. (2003). $\beta$-Lactam resistance modulated by the overexpression of response regulators of two- 
component signal transduction systems in Escherichia coli. Journal of Antimicrobial Chemotherapy, 52(4), 576-582. https://doi.org/10.1093/jac/dkg406

Hoch, J. A. (2000). Two-component and phosphorelay signal transduction. Current Opinion in Microbiology, 3(2), 165-170. https://doi.org/10.1016/S1369$\underline{5274(00) 00070-9}$

Hoch, J. A., \& Silhavy, T. J. (Eds.). (1995). Two-component signal transduction. American Society for Microbiology, Washington, D.C, 25-51. http://dx.doi.org/10.1128/9781555818319

Holmqvist, E., \& Vogel, J. (2013). A small RNA serving both the Hfq and CsrA regulons. Genes \& Development, 27(10), 1073-1078. https://doi.org/10.1101/gad.220178.113

Hooper, D. C. (1995). Bacterial Resistance to Fluoroquinolones: Mechanisms and Patterns BT - Antimicrobial Resistance: A Crisis in Health Care (D. L. Jungkind, J. E. Mortensen, H. S. Fraimow, \& G. B. Calandra, eds.). Antimicrobial Resistance. Advances in Experimental Medicine and Biology, 390, 49-57. https://doi.org/10.1007/978-1-4757-9203-4_4

Hooper, D. C. (1999). Mechanisms of fluoroquinolone resistance. Drug Resistance Updates, 2(1), 38-55. https://doi.org/10.1054/drup.1998.0068

Hooper, D. C. (2001). Emerging mechanisms of fluoroquinolone resistance. Emerging Infectious Diseases, 7(2), 337-341. https://doi.org/10.3201/eid0702.700337 
Hooper, D. C. (2001). Mechanisms of action of antimicrobials: Focus on fluoroquinolones. Clinical Infectious Diseases, 32(Supplement_1), S9-S15. https://doi.org/10.1086/319370

Hooper, D. C., \& Jacoby, G. A. (2015). Mechanisms of drug resistance: Quinolone resistance. Annals of the New York Academy of Sciences, 1354(1), 12-31. https://doi.org/10.1111/nyas. 12830

Hopkins, K. L., Davies, R. H., \& Threlfall, E. J. (2005). Mechanisms of quinolone resistance in Escherichia coli and Salmonella: Recent developments. International Journal of Antimicrobial Agents, 25(5), 358-373. https://doi.org/10.1016/j.ijantimicag.2005.02.006

Horowitz, D. S., \& Wang, J. C. (1987). Mapping the active site tyrosine of Escherichia coli DNA gyrase. Journal of Biological Chemistry, 262(11), 5339-5344. http://www.jbc.org/content/262/11/5339.abstract

Hu, W. S., Chen, H.-W., Zhang, R.-Y., Huang, C.-Y., \& Shen, C.-F. (2011). The expression levels of outer membrane proteins STM1530 and OmpD, which are influenced by the CpxAR and BaeSR two-component systems, play important roles in the ceftriaxone resistance of Salmonella enterica serovar Typhimurium. Antimicrobial Agents and Chemotherapy, 55(8), 3829-3837. https://doi.org/10.1128/AAC.00216-11 
Hughes, D., \& Andersson, D. I. (2017). Evolutionary trajectories to antibiotic resistance. Annual Review of Microbiology, 71(1), 579-596. https://doi.org/10.1146/annurev$\underline{\text { micro-090816-093813 }}$

Huseby, D. L., Pietsch, F., Brandis, G., Garoff, L., Tegehall, A., \& Hughes, D. (2017). Mutation supply and relative fitness shape the genotypes of ciprofloxacin-resistant Escherichia coli. Molecular Biology and Evolution, 34(5), 1029-1039. https://doi.org/10.1093/molbev/msx052

Imamovic, L., \& Sommer, M. O. A. (2013). Use of collateral sensitivity networks to design drug cycling protocols that avoid resistance development. Science Translational Medicine, 5(204), 204ra132. https://doi.org/10.1126/scitranslmed.3006609

Ince, D., \& Hooper, D. C. (2003). Quinolone resistance due to reduced target enzyme expression. Journal of Bacteriology, 185(23), 6883-6892. https://doi.org/10.1128/jb.185.23.6883-6892.2003

Jacoby, G. A. (2005). Mechanisms of resistance to quinolones. Clinical Infectious Diseases, 41(Supplement_2), S120-S126. https://doi.org/10.1086/428052

Jana, S., \& Deb, J. K. (2006). Molecular understanding of aminoglycoside action and resistance. Applied Microbiology and Biotechnology, 70(2), 140-150. https://doi.org/10.1007/s00253-005-0279-0 
Jorgensen, J. H., Weigel, L. M., Ferraro, M. J., Swenson, J. M., \& Tenover, F. C. (1999). Activities of newer fluoroquinolones against Streptococcus pneumoniae clinical isolates including those with mutations in the $\operatorname{gyr} A$, $\operatorname{par} C$, and $\operatorname{parE}$ loci. Antimicrobial Agents and Chemotherapy, 43(2), 329-334. https://doi.org/10.1128/AAC.43.2.329

Jousselin, A., Metzinger, L., \& Felden, B. (2009). On the facultative requirement of the bacterial RNA chaperone, Hfq. Trends in Microbiology, 17(9), 399-405. https://doi.org/10.1016/j.tim.2009.06.003

Jung, K., Fried, L., Behr, S., \& Heermann, R. (2012). Histidine kinases and response regulators in networks. Current Opinion in Microbiology, 15(2), 118-124. https://doi.org/10.1016/j.mib.2011.11.009

Kalan, L., \& Wright, G. D. (2011). Antibiotic adjuvants: Multicomponent anti-infective strategies. Expert Reviews in Molecular Medicine, 13, e5. https://doi.org/10.1017/S1462399410001766

Källman, O., Giske, C. G., Samuelsen, Ø., Wretlind, B., Kalin, M., \& Olsson-Liljequist, B. (2009). Interplay of efflux, impermeability, and AmpC activity contributes to Cefuroxime resistance in clinical, non-ESBL-producing isolates of Escherichia coli. Microbial Drug Resistance, 15(2), 91-95. https://doi.org/10.1089/mdr.2009.0883

Kampranis, S. C., Bates, A. D., \& Maxwell, A. (1999). A model for the mechanism of strand passage by DNA gyrase. Proceedings of the National Academy of Sciences 
of the United States of America, 96(15), 8414-8419.

https://doi.org/10.1073/pnas.96.15.8414

Kato, A., \& Groisman, E. A. (2008). The PhoQ/PhoP regulatory network of Salmonella enterica BT-Bacterial Signal Transduction: Networks and Drug Targets (R.

Utsumi, Ed.), 631, 7-21. https://doi.org/10.1007/978-0-387-78885-2 2

Kato, A., Tanabe, H., \& Utsumi, R. (1999). Molecular characterization of the PhoP-PhoQ two-component system in Escherichia coli K-12: Identification of extracellular Mg2+-responsive promoters. Journal of Bacteriology, 181(17), 5516-5520. https://www.ncbi.nlm.nih.gov/pubmed/10464230

Kery, M. B., Feldman, M., Livny, J., \& Tjaden, B. (2014). TargetRNA2: identifying targets of small regulatory RNAs in bacteria. Nucleic Acids Research, 42(Web Server issue), W124-W129. https://doi.org/10.1093/nar/gku317

Keseler, I. M., Mackie, A., Santos-Zavaleta, A., Billington, R., Bonavides-Martínez, C., Caspi, R., ... Karp, P. D. (2017). The EcoCyc database: Reflecting new knowledge about Escherichia coli K-12. Nucleic Acids Research, 45(D1), D543-D550. https://doi.org/10.1093/nar/gkw1003

Kibota, T. T., \& Lynch, M. (1996). Estimate of the genomic mutation rate deleterious to overall fitness in E. coli. Nature, 381(6584), 694-696.

https://doi.org/10.1038/381694a0 
King, A. M., Reid-Yu, S. A., Wang, W., King, D. T., De Pascale, G., Strynadka, N. C., ... Wright, G. D. (2014). Aspergillomarasmine A overcomes metallo- $\beta$-lactamase antibiotic resistance. Nature, 510(7506), 503-506.

https://doi.org/10.1038/nature13445

Knopp, M., \& Andersson, D. I. (2015). Amelioration of the fitness costs of antibiotic resistance due to reduced outer membrane permeability by upregulation of alternative porins. Molecular Biology and Evolution, 32(12), 3252-3263. https://doi.org/10.1093/molbev/msv195

Kocsis, B., Domokos, J., \& Szabo, D. (2016). Chemical structure and pharmacokinetics of novel quinolone agents represented by avarofloxacin, delafloxacin, finafloxacin, zabofloxacin and nemonoxacin. Annals of Clinical Microbiology and Antimicrobials, 15(1), 34. https://doi.org/10.1186/s12941-016-0150-4

Komp Lindgren, K., Marcusson, L. L., Sandvang, D., Frimodt-Møller, N., \& Hughes, D. (2005). Biological cost of single and multiple norfloxacin resistance mutations in Escherichia coli implicated in urinary tract infections. Antimicrobial Agents and Chemotherapy, 49(6), 2343-2351.https://doi.org/10.1128/AAC.49.6.2343$\underline{2351.2005}$

Komp Lindgren, P., Karlsson, A., \& Hughes, D. (2003). Mutation rate and evolution of fluoroquinolone resistance in Escherichia coli isolates from patients with urinary tract infections. Antimicrobial Agents and Chemotherapy, 47(10), 3222-3232. https://doi.org/10.1128/aac.47.10.3222-3232.2003 
Kraxenberger, T., Fried, L., Behr, S., \& Jung, K. (2012). First insights into the unexplored two-component system YehU/YehT in Escherichia coli. Journal of Bacteriology, 194(16), 4272-4284. https://doi.org/10.1128/JB.00409-12

Krell, T., Lacal, J., Busch, A., Silva-Jiménez, H., Guazzaroni, M. E., \& Ramos, J. L. (2010). Bacterial sensor kinases: Diversity in the recognition of environmental signals. Annual Review of Microbiology, 64(1), 539-559. https://doi.org/10.1146/annurev.micro.112408.134054

Kugelberg, E., Löfmark, S., Wretlind, B., \& Andersson, D. I. (2005). Reduction of the fitness burden of quinolone resistance in Pseudomonas aeruginosa. Journal of Antimicrobial Chemotherapy, 55(1), 22-30. https://doi.org/10.1093/jac/dkh505

Kurabayashi, K., Hirakawa, Y., Tanimoto, K., Tomita, H., \& Hirakawa, H. (2014). Role of the CpxAR two-component signal transduction system in control of fosfomycin resistance and carbon substrate uptake. Journal of Bacteriology, 196(2), 248-256. https://doi.org/10.1128/JB.01151-13

Labby, K. J., \& Garneau-Tsodikova, S. (2013). Strategies to overcome the action of aminoglycoside-modifying enzymes for treating resistant bacterial infections. Future Medicinal Chemistry, 5(11), 1285-1309. https://doi.org/10.4155/fmc.13.80

Lambert, P. A. (2005). Bacterial resistance to antibiotics: Modified target sites. Advanced Drug Delivery Reviews, 57(10), 1471-1485.

https://doi.org/10.1016/j.addr.2005.04.003 
Langmead, B., Trapnell, C., Pop, M., \& Salzberg, S. L. (2009). Ultrafast and memoryefficient alignment of short DNA sequences to the human genome. Genome Biology, 10(3), R25-R25. https://doi.org/10.1186/gb-2009-10-3-r25

Laub, M. T., \& Goulian, M. (2007). Specificity in two-component signal transduction pathways. Annual Review of Genetics, 41(1), 121-145. https://doi.org/10.1146/annurev.genet.41.042007.170548

Lázár, V., Pal Singh, G., Spohn, R., Nagy, I., Horváth, B., Hrtyan, M., ... Pál, C. (2013). Bacterial evolution of antibiotic hypersensitivity. Molecular Systems Biology, 9, 700. https://doi.org/10.1038/msb.2013.57

Lea, D. E., \& Coulson, C. A. (1949). The distribution of the numbers of mutants in bacterial populations. Journal of Genetics, 49(3), 264.

https://doi.org/10.1007/BF02986080

LeBel, M. (1988). Ciprofloxacin: Chemistry, mechanism of action, resistance, antimicrobial spectrum, pharmacokinetics, clinical trials, and adverse reactions. Pharmacotherapy: The Journal of Human Pharmacology and Drug Therapy, 8(1), 3-30. https://doi.org/10.1002/j.1875-9114.1988.tb04058.x

Leblanc, S. K. D., Oates, C. W., \& Raivio, T. L. (2011). Characterization of the induction and cellular role of the BaeSR two-component envelope stress response of Escherichia coli. Journal of Bacteriology, 193(13), 3367-3375. https://doi.org/10.1128/JB.01534-10 
Lee, S. H., Jarantow, L. W., Wang, H., Sillaots, S., Cheng, H., Meredith, T. C., ... Roemer, T. (2011). Antagonism of chemical genetic interaction networks resensitize MRSA to $\beta$-lactam antibiotics. Chemistry \& Biology, 18(11), 13791389. https://doi.org/10.1016/j.chembiol.2011.08.015

Lee, S., Hinz, A., Bauerle, E., Angermeyer, A., Juhaszova, K., Kaneko, Y., ... Manoil, C. (2009). Targeting a bacterial stress response to enhance antibiotic action. Proceedings of the National Academy of Sciences, 106(34), 14570-14575. https://doi.org/10.1073/pnas.0903619106

Lee, Y. J., \& Moon, T. S. (2018). Design rules of synthetic non-coding RNAs in bacteria. Methods, 143, 58-69. https://doi.org/10.1016/j.ymeth.2018.01.001

Lee, Y. J., Kim, S.-J., Amrofell, M. B., \& Moon, T. S. (2019). Establishing a multivariate model for predictable antisense RNA-mediated repression. ACS Synthetic Biology, 8(1), 45-56. https://doi.org/10.1021/acssynbio.8b00227

Lehner, B. (2011). Molecular mechanisms of epistasis within and between genes. Trends in Genetics, 27(8), 323-331. https://doi.org/10.1016/j.tig.2011.05.007

Lennen, R. M., Nilsson Wallin, A. I., Pedersen, M., Bonde, M., Luo, H., Herrgård, M. J., \& Sommer, M. O. A. (2016). Transient overexpression of DNA adenine methylase enables efficient and mobile genome engineering with reduced off-target effects. Nucleic Acids Research, 44(4), e36. https://doi.org/10.1093/nar/gkv1090 
Lesher, G. Y., Froelich, E. J., Gruett, M. D., Bailey, J. H., \& Brundage, R. P. (1962). 1,8naphthyridine derivatives. A new class of chemotherapeutic agents. Journal of Medicinal and Pharmaceutical Chemistry, 5(5), 1063-1065.

https://doi.org/10.1021/jm01240a021

Levin, B. R., \& Rozen, D. E. (2006). Non-inherited antibiotic resistance. Nature Reviews Microbiology, 4(7), 556-562. https://doi.org/10.1038/nrmicro1445

Levin, B. R., Perrot, V., \& Walker, N. (2000). Compensatory mutations, antibiotic resistance and the population genetics of adaptive evolution in bacteria. Genetics, 154(3), 985-997. https://www.ncbi.nlm.nih.gov/pubmed/10757748

Levy, S. B., \& Marshall, B. (2004). Antibacterial resistance worldwide: causes, challenges and responses. Nature Medicine, 10, S122-S129. $\underline{\text { https://doi.org/10.1038/nm1145 }}$

Li, H., Handsaker, B., Wysoker, A., Fennell, T., Ruan, J., Homer, N., ... Subgroup, 1000 Genome Project Data Processing. (2009). The sequence alignment/map format and SAMtools. Bioinformatics (Oxford, England), 25(16), 2078-2079.

https://doi.org/10.1093/bioinformatics/btp352

Li, X. Z., Plésiat, P., \& Nikaido, H. (2015). The challenge of efflux-mediated antibiotic resistance in Gram-negative bacteria. Clinical Microbiology Reviews, 28(2), 337418. https://doi.org/10.1128/CMR.00117-14 
Lin, M. F., Lin, Y. Y., Yeh, H. W., \& Lan, C. Y. (2014). Role of the BaeSR twocomponent system in the regulation of Acinetobacter baumannii adeAB genes and its correlation with tigecycline susceptibility. BMC Microbiology, 14(1), 119. https://doi.org/10.1186/1471-2180-14-119

Ling, L. L., Schneider, T., Peoples, A. J., Spoering, A. L., Engels, I., Conlon, B. P., ... Lewis, K. (2015). A new antibiotic kills pathogens without detectable resistance. Nature, 517, 455-459. https://doi.org/10.1038/nature14098

Liu, A., Tran, L., Becket, E., Lee, K., Chinn, L., Park, E., ... Miller, J. H. (2010). Antibiotic sensitivity profiles determined with an Escherichia coli gene knockout collection: generating an antibiotic bar code. Antimicrobial Agents and Chemotherapy, 54(4), 1393-1403. https://doi.org/10.1128/AAC.00906-09

Livermore, D. M., Development, on behalf of the B. S. for A. C. W. P. on T. U. N. R. A. D. D. and, Blaser, M., Carrs, O., Cassell, G., Fishman, N., ... White, T. (2011). Discovery research: The scientific challenge of finding new antibiotics. Journal of Antimicrobial Chemotherapy, 66(9), 1941-1944. https://doi.org/10.1093/jac/dkr262

Lomovskaya, O., Lewis, K., \& Matin, A. (1995). EmrR is a negative regulator of the Escherichia coli multidrug resistance pump EmrAB. Journal of Bacteriology, 177(9), 2328-2334. https://doi.org/10.1128/jb.177.9.2328-2334.1995

Lu, T. K., \& Collins, J. J. (2009). Engineered bacteriophage targeting gene networks as adjuvants for antibiotic therapy. Proceedings of the National Academy of Sciences 
of the United States of America, 106(12), 4629-4634.

https://doi.org/10.1073/pnas.0800442106

Lu, T., Zhao, X., \& Drlica, K. (1999). Gatifloxacin activity against quinolone-resistant gyrase: Allele-specific enhancement of bacteriostatic and bactericidal activities by the C-8-methoxy group. Antimicrobial Agents and Chemotherapy, 43(12), 29692974. https://doi.org/10.1128/AAC.43.12.2969

Luo, N., Pereira, S., Sahin, O., Lin, J., Huang, S., Michel, L., \& Zhang, Q. (2005). Enhanced in vivo fitness of fluoroquinolone-resistant Campylobacter jejuni in the absence of antibiotic selection pressure. Proceedings of the National Academy of Sciences of the United States of America, 102(3), 541-546. https://doi.org/10.1073/pnas.0408966102

Luria, S. E., \& Delbrück, M. (1943). Mutations of bacteria from virus sensitivity to virus resistance. Genetics, 28(6), 491-511.

http://www.genetics.org/content/28/6/491.abstract

Ma, W. T., Sandri, G. H., \& Sarkar, S. (1992). Analysis of the Luria-Delbrück distribution using discrete convolution powers. Journal of Applied Probability, 29(2), 255-267. https://doi.org/10.2307/3214564

Macfarlane, E. L. A., Kwasnicka, A., \& Hancock, R. E. W. (2000). Role of Pseudomonas aeruginosa PhoP-PhoQ in resistance to antimicrobial cationic peptides and aminoglycosides. Microbiology, 146(10), 2543-2554.

https://dx.doi.org/10.1099/00221287-146-10-2543 
Macielag, M. J., \& Goldschmidt, R. (2000). Inhibitors of bacterial two-component signalling systems. Expert Opinion on Investigational Drugs, 9(10), 2351-2369. $\underline{\text { https://doi.org/10.1517/13543784.9.10.2351 }}$

MacLean, R. C., \& Vogwill, T. (2014). Limits to compensatory adaptation and the persistence of antibiotic resistance in pathogenic bacteria. Evolution, Medicine, and Public Health, 2015(1), 4-12. https://doi.org/10.1093/emph/eou032

Macvanin, M., Ballagi, A., \& Hughes, D. (2004). Fusidic acid-resistant mutants of Salmonella enterica serovar typhimurium have low levels of heme and a reduced rate of respiration and are sensitive to oxidative stress. Antimicrobial Agents and Chemotherapy, 48(10), 3877-3883. https://doi.org/10.1128/AAC.48.10.3877$\underline{3883.2004}$

Macvanin, M., Björkman, J., Eriksson, S., Rhen, M., Andersson, D. I., \& Hughes, D. (2003). Fusidic acid-resistant mutants of Salmonella enterica serovar Typhimurium with low fitness in vivo are defective in RpoS induction. Antimicrobial Agents and Chemotherapy, 47(12), 3743-3749. https://doi.org/10.1128/aac.47.12.3743$\underline{3749.2003}$

Mahoney, T. F., \& Silhavy, T. J. (2013). The Cpx stress response confers resistance to some, but not all, bactericidal antibiotics. Journal of Bacteriology, 195(9), 18691874. https://doi.org/10.1128/JB.02197-12 
Maisnier-Patin, S., \& Andersson, D. I. (2004). Adaptation to the deleterious effects of antimicrobial drug resistance mutations by compensatory evolution. Research in Microbiology, 155(5), 360-369. https://doi.org/10.1016/j.resmic.2004.01.019

Maisnier-Patin, S., Berg, O. G., Liljas, L., \& Andersson, D. I. (2002). Compensatory adaptation to the deleterious effect of antibiotic resistance in Salmonella typhimurium. Molecular Microbiology, 46(2), 355-366. https://doi.org/10.1046/j.1365-2958.2002.03173.x

Malachowa, N., \& DeLeo, F. R. (2010). Mobile genetic elements of Staphylococcus aureus. Cellular and Molecular Life Sciences : CMLS, 67(18), 3057-3071. https://doi.org/10.1007/s00018-010-0389-4

Man, S., Cheng, R., Miao, C., Gong, Q., Gu, Y., Lu, X., ... Yu, W. (2011). Artificial trans-encoded small non-coding RNAs specifically silence the selected gene expression in bacteria. Nucleic Acids Research, 39(8), e50.

https://doi.org/10.1093/nar/gkr034

Mann, M., Wright, P. R., \& Backofen, R. (2017). IntaRNA 2.0: enhanced and customizable prediction of RNA-RNA interactions. Nucleic Acids Research, 45(W1), W435-W439. https://doi.org/10.1093/nar/gkx279

Marchand, I., Damier-Piolle, L., Courvalin, P., \& Lambert, T. (2004). Expression of the RND-type efflux pump AdeABC in Acinetobacter baumannii is regulated by the AdeRS two-component system. Antimicrobial Agents and Chemotherapy, 48(9), 3298-3304. https://doi.org/10.1128/AAC.48.9.3298-3304.2004 
Marcusson, L. L., Frimodt-Møller, N., \& Hughes, D. (2009). Interplay in the selection of fluoroquinolone resistance and bacterial fitness. PLOS Pathogens, 5(8), e1000541. https://doi.org/10.1371/journal.ppat.1000541

Martínez-Martínez, L., Pascual, A., \& Jacoby, G. A. (1998). Quinolone resistance from a transferable plasmid. The Lancet, 351(9105), 797-799. https://doi.org/10.1016/S0140-6736(97)07322-4

Martínez, J. L. (2012). The antibiotic resistome: Challenge and opportunity for therapeutic intervention. Future Medicinal Chemistry, 4(3), 347-359. $\underline{\text { https://doi.org/10.4155/fmc.12.2 }}$

Martinez, J. L., \& Baquero, F. (2000). Mutation frequencies and antibiotic resistance. Antimicrobial Agents and Chemotherapy, 44(7), 1771-1777. $\underline{\text { https://doi.org/10.1128/AAC.44.7.1771-1777.2000 }}$

Martínez, J. L., \& Rojo, F. (2011). Metabolic regulation of antibiotic resistance. FEMS Microbiology Reviews, 35(5), 768-789. https://doi.org/10.1111/j.1574$\underline{6976.2011 .00282 . x}$

Martínez, J. L., Baquero, F., \& Andersson, D. I. (2007). Predicting antibiotic resistance. Nature Reviews Microbiology, 5, 958-965. https://doi.org/10.1038/nrmicro1796

Martínez, J. L., Baquero, F., \& Andersson, D. I. (2011). Beyond serial passages: New methods for predicting the emergence of resistance to novel antibiotics. Current 
Opinion in Pharmacology, 11(5), 439-445.

https://doi.org/10.1016/j.coph.2011.07.005

Martinez, J. L., Fajardo, A., Garmendia, L., Hernandez, A., Linares, J. F., Martínez-

Solano, L., \& Sánchez, M. B. (2009). A global view of antibiotic resistance. FEMS

Microbiology Reviews, 33(1), 44-65. https://doi.org/10.1111/j.1574-

$\underline{6976.2008 .00142 . x}$

Mascher, T., Helmann, J. D., \& Unden, G. (2006). Stimulus perception in bacterial signal-transducing histidine kinases. Microbiology and Molecular Biology Reviews : MMBR, 70(4), 910-938. https://doi.org/10.1128/MMBR.00020-06

Masterton, R. G. (2005). Antibiotic cycling: more than it might seem? Journal of Antimicrobial Chemotherapy, 55(1), 1-5. https://doi.org/10.1093/jac/dkh506

Matson, S. W., \& Robertson, A. B. (2006). The UvrD helicase and its modulation by the mismatch repair protein MutL. Nucleic Acids Research, 34(15), 4089-4097. https://doi.org/10.1093/nar/gk1450

Maxwell, A. (1992). The molecular basis of quinolone action. Journal of Antimicrobial Chemotherapy, 30(4), 409-414. https://doi.org/10.1093/jac/30.4.409

Mayer, M., Rüdiger, S. \& Bukau, B. (2005). Molecular basis for interactions of the DnaK chaperone with substrates. Biological Chemistry, 381(9-10), 877-885.

http://doi:10.1515/BC.2000.109 
McPhee, J. B., Lewenza, S., \& Hancock, R. E. W. (2003). Cationic antimicrobial peptides activate a two-component regulatory system, PmrA-PmrB, that regulates resistance to polymyxin B and cationic antimicrobial peptides in Pseudomonas aeruginosa. Molecular Microbiology, 50(1), 205-217. https://doi.org/10.1046/j.1365-2958.2003.03673.x

Melander, R. J., \& Melander, C. (2017). The challenge of overcoming antibiotic resistance: An adjuvant approach? ACS Infectious Diseases, 3(8), 559-563. https://doi.org/10.1021/acsinfecdis.7b00071

Melnyk, A. H., Wong, A., \& Kassen, R. (2015). The fitness costs of antibiotic resistance mutations. Evolutionary Applications, 8(3), 273-283. https://doi.org/10.1111/eva.12196

Mendonca, V. M., Kaiser-Rogers, K., \& Matson, S. W. (1993). Double helicase II (uvrD)-helicase IV (helD) deletion mutants are defective in the recombination pathways of Escherichia coli. Journal of Bacteriology, 175(15), 4641-4651. https://doi.org/10.1128/jb.175.15.4641-4651.1993

Meng, J., Kanzaki, G., Meas, D., Lam, C. K., Crummer, H., Tain, J., \& Xu, H. H. (2012). A genome-wide inducible phenotypic screen identifies antisense RNA constructs silencing Escherichia coli essential genes. FEMS Microbiology Letters, 329(1), 4553. https://doi.org/10.1111/j.1574-6968.2012.02503.x

Menon, N. K., Chatelus, C. Y., Dervartanian, M., Wendt, J. C., Shanmugam, K. T., Peck, H. D., \& Przybyla, A. E. (1994). Cloning, sequencing, and mutational analysis of 
the hyb operon encoding Escherichia coli hydrogenase 2. Journal of Bacteriology, 176(14), 4416-4423. https://doi.org/10.1128/jb.176.14.4416-4423.1994

Michot, J.-M., Seral, C., Van Bambeke, F., Mingeot-Leclercq, M.-P., \& Tulkens, P. M. (2005). Influence of efflux transporters on the accumulation and efflux of four quinolones (ciprofloxacin, levofloxacin, garenoxacin, and moxifloxacin) in J774 macrophages. Antimicrobial Agents and Chemotherapy, 49(6), 2429-2437. https://doi.org/10.1128/AAC.49.6.2429-2437.2005

Mitscher, L. A. (2005). Bacterial topoisomerase inhibitors: Quinolone and Pyridone antibacterial agents. Chemical Reviews, 105(2), 559-592.

https://doi.org/10.1021/cr030101q

Mizuno, T. (1997). Compilation of all genes encoding two-component phosphotransfer signal transducers in the genome of Escherichia coli. DNA Research, 4(2), 161168. https://doi.org/10.1093/dnares/4.2.161

Møller, T., Franch, T., Højrup, P., Keene, D. R., Bächinger, H. P., Brennan, R. G., \& Valentin-Hansen, P. (2002). Hfq: A bacterial Sm-like protein that mediates RNARNA interaction. Molecular Cell, 9(1), 23-30. https://doi.org/10.1016/S1097$\underline{2765(01) 00436-1}$

Montero, M., Eydallin, G., Viale, A. M., Almagro, G., Muñoz, F. J., Rahimpour, M., ... Pozueta-Romero, J. (2009). Escherichia coli glycogen metabolism is controlled by the PhoP-PhoQ regulatory system at submillimolar environmental $\mathrm{Mg}$ concentrations, and is highly interconnected with a wide variety of cellular 
processes. Biochemical Journal, 424(1), 129-141.

https://doi.org/10.1042/BJ20090980

Moon, D. C., Seol, S. Y., Gurung, M., Jin, J. S., Choi, C. H., Kim, J., ... Lee, J. C. (2010). Emergence of a new mutation and its accumulation in the topoisomerase IV gene confers high levels of resistance to fluoroquinolones in Escherichia coli isolates. International Journal of Antimicrobial Agents, 35(1), 76-79. https://doi.org/10.1016/j.ijantimicag.2009.08.003

Moon, K., \& Gottesman, S. (2009). A PhoQ/P-regulated small RNA regulates sensitivity of Escherichia coli to antimicrobial peptides. Molecular Microbiology, 74(6), 1314-1330. https://doi.org/10.1111/j.1365-2958.2009.06944.x

Morgan-Linnell, S. K., Becnel Boyd, L., Steffen, D., \& Zechiedrich, L. (2009). Mechanisms accounting for fluoroquinolone resistance in Escherichia coli clinical isolates. Antimicrobial Agents and Chemotherapy, 53(1), 235-241. https://doi.org/10.1128/AAC.00665-08

Mulcahy, H., Charron-Mazenod, L., \& Lewenza, S. (2008). Extracellular DNA chelates cations and induces antibiotic resistance in Pseudomonas aeruginosa biofilms. PLOS Pathogens, 4(11), e1000213. https://doi.org/10.1371/journal.ppat.1000213

Munita, J. M., \& Arias, C. A. (2016). Mechanisms of antibiotic resistance. Microbiology Spectrum, 4(2), 481-511. https://doi.org/10.1128/microbiolspec.VMBF-0016-2015 
Nagaev, I., Björkman, J., Andersson, D. I., \& Hughes, D. (2001). Biological cost and compensatory evolution in fusidic acid-resistant Staphylococcus aureus. Molecular Microbiology, 40(2), 433-439. https://doi.org/10.1046/j.1365-2958.2001.02389.x

Nakamura, S., Nakamura, M., Kojima, T., \& Yoshida, H. (1989). gyrA and gyrB mutations in quinolone-resistant strains of Escherichia coli. Antimicrobial Agents and Chemotherapy, 33(2), 254-255. https://doi.org/10.1128/aac.33.2.254

Nakanishi, A., Oshida, T., Matsushita, T., Imajoh-Ohmi, S., \& Ohnuki, T. (1998). Identification of DNA gyrase inhibitor (GyrI) in Escherichia coli. Journal of Biological Chemistry, 273(4), 1933-1938. http://doi.org/10.1074/jbc.273.4.1933

Nakashima, N., Tamura, T., \& Good, L. (2006). Paired termini stabilize antisense RNAs and enhance conditional gene silencing in Escherichia coli. Nucleic Acids Research, 34(20), e138. https://doi.org/10.1093/nar/gk1697

Nale, J. Y., Spencer, J., Hargreaves, K. R., Buckley, A. M., Trzepiński, P., Douce, G. R., \& Clokie, M. R. J. (2015). Bacteriophage combinations significantly reduce Clostridium difficile growth in vitro and proliferation in vivo. Antimicrobial Agents and Chemotherapy, 60(2), 968-981. https://doi.org/10.1128/AAC.01774-15

Needham, B. D., \& Trent, M. S. (2013). Fortifying the barrier: The impact of lipid A remodelling on bacterial pathogenesis. Nature Reviews Microbiology, 11, 467-481. https://doi.org/10.1038/nrmicro3047 
Nikaido, H. (1989). Outer membrane barrier as a mechanism of antimicrobial resistance. Antimicrobial Agents and Chemotherapy, 33(11), 1831-1836. https://doi.org/10.1128/aac.33.11.1831

Nikaido, H. (1994). Prevention of drug access to bacterial targets: Permeability barriers and active efflux. Science, 264(5157), 382-388. $\underline{\text { https://doi.org/10.1126/science.8153625 }}$

Nikaido, H. (1996). Multidrug efflux pumps of gram-negative bacteria. Journal of Bacteriology, 178(20), 5853-5859. https://doi.org/10.1128/jb.178.20.5853$\underline{5859.1996}$

Nikaido, H. (2003). Molecular basis of bacterial outer membrane permeability revisited. Microbiology and Molecular Biology Reviews : MMBR, 67(4), 593-656. https://doi.org/10.1128/mmbr.67.4.593-656.2003

Nikaido, H., \& Thanassi, D. G. (1993). Penetration of lipophilic agents with multiple protonation sites into bacterial cells: Tetracyclines and fluoroquinolones as examples. Antimicrobial Agents and Chemotherapy, 37(7), 1393-1399. https://doi.org/10.1128/aac.37.7.1393

Nishino, K., \& Yamaguchi, A. (2001). Analysis of a complete library of putative drug transporter genes in Escherichia coli. Journal of Bacteriology, 183(20), 5803-5812. https://doi.org/10.1128/JB.183.20.5803-5812.2001 
Nishino, K., \& Yamaguchi, A. (2002). EvgA of the two-component signal transduction system modulates production of the $y h i U V$ multidrug transporter in Escherichia coli. Journal of Bacteriology, 184(8), 2319-2323.

https://doi.org/10.1128/jb.184.8.2319-2323.2002

O’Neill, A. J., Huovinen, T., Fishwick, C. W. G., \& Chopra, I. (2006). Molecular genetic and structural modeling studies of Staphylococcus aureus RNA polymerase and the fitness of rifampin resistance genotypes in relation to clinical prevalence.

Antimicrobial Agents and Chemotherapy, 50(1), 298-309.

$\underline{\text { https://doi.org/10.1128/AAC.50.1.298-309.2006 }}$

O’Neill, J. (2014). Antimicrobial resistance: tackling a crisis for the health and wealth of nations. Rev Antimicrob Resist. http://amr-review.org/Publications.

Ochman, H., Lawrence, J. G., \& Groisman, E. A. (2000). Lateral gene transfer and the nature of bacterial innovation. Nature, 405(6784), 299-304.

https://doi.org/10.1038/35012500

Olivares Pacheco, J., Alvarez-Ortega, C., Alcalde Rico, M., \& Martínez, J. L. (2017). Metabolic compensation of fitness costs is a general outcome for antibioticresistant Pseudomonas aeruginosa mutants overexpressing efflux pumps. MBio, 8(4), e00500-17. https://doi.org/10.1128/mBio.00500-17

Olivares, J., Bernardini, A., Garcia-Leon, G., Corona, F., B Sanchez, M., \& Martinez, J. L. (2013). The intrinsic resistome of bacterial pathogens. Frontiers in Microbiology, 4, 103. https://doi.org/10.3389/fmicb.2013.00103 
Olivera, B. C. L., Ugalde, E., \& Martínez-Antonio, A. (2010). Regulatory dynamics of standard two-component systems in bacteria. Journal of Theoretical Biology, 264(2), 560-569. https://doi.org/10.1016/j.jtbi.2010.02.008

Oram, M., \& Fisher, L. M. (1991). 4-Quinolone resistance mutations in the DNA gyrase of Escherichia coli clinical isolates identified by using the polymerase chain reaction. Antimicrobial Agents and Chemotherapy, 35(2), 387-389. https://doi.org/10.1128/aac.35.2.387

Oshima, T., Aiba, H., Masuda, Y., Kanaya, S., Sugiura, M., Wanner, B. L., ... Mizuno, T. (2002). Transcriptome analysis of all two-component regulatory system mutants of Escherichia coli K-12. Molecular Microbiology, 46(1), 281-291. https://doi.org/10.1046/j.1365-2958.2002.03170.x

Pagès, J.-M., James, C. E., \& Winterhalter, M. (2008). The porin and the permeating antibiotic: a selective diffusion barrier in Gram-negative bacteria. Nature Reviews Microbiology, 6, 893-903. https://doi.org/10.1038/nrmicro1994

Pál, C., Papp, B., \& Lázár, V. (2015). Collateral sensitivity of antibiotic-resistant microbes. Trends in Microbiology, 23(7), 401-407. https://doi.org/10.1016/j.tim.2015.02.009

Pallecchi, L., Bartoloni, A., Riccobono, E., Fernandez, C., Mantella, A., Magnelli, D., ... Rossolini, G. M. (2012). Quinolone resistance in absence of selective pressure: The experience of a very remote community in the amazon forest. PLOS Neglected Tropical Diseases, 6(8), e1790. https://doi.org/10.1371/journal.pntd.0001790 
Palumbi, S. R. (2001). Humans as the world's greatest evolutionary force. Science, 293(5536), 1786-1790. https://doi.org/10.1126/science.293.5536.1786

Park, C. H., Robicsek, A., Jacoby, G. A., Sahm, D., \& Hooper, D. C. (2006). Prevalence in the United States of aac(6')-Ib-cr encoding a ciprofloxacin-modifying enzyme. Antimicrobial Agents and Chemotherapy, 50(11), 3953-3955.

https://doi.org/10.1128/AAC.00915-06

Parkinson, J. S. (1993). Signal transduction schemes of bacteria. Cell, 73(5), 857-871. https://doi.org/10.1016/0092-8674(93)90267-T

Paulander, W., Maisnier-Patin, S., \& Andersson, D. I. (2009). The fitness cost of streptomycin resistance depends on rpsL mutation, carbon source and RpoS (sigmaS). Genetics, 183(2), 539-546. https://doi.org/10.1534/genetics.109.106104

Payne, D. J., Gwynn, M. N., Holmes, D. J., \& Pompliano, D. L. (2006). Drugs for bad bugs: Confronting the challenges of antibacterial discovery. Nature Reviews Drug Discovery, 6, 29-40. https://doi.org/10.1038/nrd2201

Pegu, A., Hessell, A. J., Mascola, J. R., \& Haigwood, N. L. (2017). Use of broadly neutralizing antibodies for HIV-1 prevention. Immunological Reviews, 275(1), 296-312. https://doi.org/10.1111/imr.12511

Périchon, B., Courvalin, P., \& Galimand, M. (2007). Transferable resistance to aminoglycosides by methylation of G1405 in 16S rRNA and to hydrophilic fluoroquinolones by QepA-mediated efflux in Escherichia coli. Antimicrobial 
Agents and Chemotherapy, 51(7), 2464-2469. https://doi.org/10.1128/AAC.00143-

$\underline{07}$

Persky, N. S., \& Lovett, S. T. (2008). Mechanisms of recombination: Lessons from E. coli. Critical Reviews in Biochemistry and Molecular Biology, 43(6), 347-370. https://doi.org/10.1080/10409230802485358

Pestova, E., Millichap, J. J., Noskin, G. A., \& Peterson, L. R. (2000). Intracellular targets of moxifloxacin: A comparison with other fluoroquinolones. Journal of Antimicrobial Chemotherapy, 45(5), 583-590. https://doi.org/10.1093/jac/45.5.583

Peterson, L. R. (2001). Quinolone molecular structure-activity relationships: What we have learned about improving antimicrobial activity. Clinical Infectious Diseases, 33(Supplement_3), S180-S186. https://doi.org/10.1086/321846

Pichoff, S., \& Lutkenhaus, J. (2001). Escherichia coli division inhibitor MinCD blocks septation by preventing Z-ring formation. Journal of Bacteriology, 183(22), 66306635. http://doi.org/10.1128/JB.183.22.6630-6635.2001

Piddock, L. J. V. (1999). Mechanisms of fluoroquinolone resistance: An update 19941998. Drugs, 58(2), 11-18. https://doi.org/10.2165/00003495-199958002-00003

Piddock, L. J. V. (2012). The crisis of no new antibiotics--what is the way forward? The Lancet Infectious Diseases, 12(3), 249-253. https://doi.org/10.1016/S1473$\underline{3099(11) 70316-4}$ 
Piddock, L. J. V. (2014). Understanding the basis of antibiotic resistance: A platform for drug discovery. Microbiology, 160(11), 2366-2373.

https://dx.doi.org/10.1099/mic.0.082412-0

Piddock, L. J. V. (2015). Teixobactin, the first of a new class of antibiotics discovered by iChip technology? Journal of Antimicrobial Chemotherapy, 70(10), 2679-2680. https://doi.org/10.1093/jac/dkv175

Pommier, Y., Leo, E., Zhang, H., \& Marchand, C. (2010). DNA topoisomerases and their poisoning by anticancer and antibacterial drugs. Chemistry \& Biology, 17(5), 421433. https://doi.org/10.1016/j.chembiol.2010.04.012

Poole, K. (2004). Efflux-mediated multiresistance in Gram-negative bacteria. Clinical Microbiology and Infection, 10(1), 12-26. https://doi.org/10.1111/j.1469$\underline{0691.2004 .00763 . x}$

Poole, K. (2004). Resistance to $\beta$-lactam antibiotics. Cell and Molecular Life Sciences, 61(17), 2200-2223. https://doi.org/10.1007/s00018-004-4060-9

Poole, K. (2005). Efflux-mediated antimicrobial resistance. Journal of Antimicrobial Chemotherapy, 56(1), 20-51. https://doi.org/10.1093/jac/dki171

Poole, K. (2012). Bacterial stress responses as determinants of antimicrobial resistance. Journal of Antimicrobial Chemotherapy, 67(9), 2069-2089. https://doi.org/10.1093/jac/dks196 
Poole, K. (2014). Stress responses as determinants of antimicrobial resistance in Pseudomonas aeruginosa: Multidrug efflux and more. Canadian Journal of Microbiology, 60(12), 783-791. https://doi.org/10.1139/cjm-2014-0666

Popa, O., \& Dagan, T. (2011). Trends and barriers to lateral gene transfer in prokaryotes. Current Opinion in Microbiology, 14(5), 615-623. https://doi.org/10.1016/j.mib.2011.07.027

Public health agency of Canada (PHAC) (2015-2016). http://www.phacaspc.gc.ca/rpp/index-eng.php.

R: A language and environment for statistical computing. R Foundation for Statistical Computing. (2018). Vienna, Austria. https://www.r-project.org/

Raffa, R. G., \& Raivio, T. L. (2002). A third envelope stress signal transduction pathway in Escherichia coli. Molecular Microbiology, 45(6), 1599-1611. https://doi.org/10.1046/j.1365-2958.2002.03112.x

Raivio, T. L. (2014). Everything old is new again: An update on current research on the Cpx envelope stress response. Biochimica et Biophysica Acta (BBA) - Molecular Cell Research, 1843(8), 1529-1541. https://doi.org/10.1016/j.bbamcr.2013.10.018

Ram, S., \& Goulian, M. (2013). The architecture of a prototypical bacterial signaling circuit enables a single point mutation to confer novel network properties. PLOS Genetics, 9(8), e1003706. https://doi.org/10.1371/journal.pgen.1003706 
Ramirez, M. S., \& Tolmasky, M. E. (2010). Aminoglycoside modifying enzymes. Drug Resistance Updates : Reviews and Commentaries in Antimicrobial and Anticancer Chemotherapy, 13(6), 151-171. https://doi.org/10.1016/j.drup.2010.08.003

Randall, L. P., \& Woodward, M. J. (2002). The multiple antibiotic resistance (mar) locus and its significance. Research in Veterinary Science, 72(2), 87-93. https://doi.org/10.1053/rvsc.2001.0537

Rasko, D. A., Moreira, C. G., Li, D. R., Reading, N. C., Ritchie, J. M., Waldor, M. K., ... Sperandio, V. (2008). Targeting QseC signaling and virulence for antibiotic development. Science (New York, N.Y.), 321(5892), 1078-1080. $\underline{\text { https://doi.org/10.1126/science. } 1160354}$

Rastogi, V. P., Verma, N., Mishra, A. K., Nath, G., Gaur, P., K., Verma, A. (2018). An Overview on Bacteriophages: A Natural Nanostructured Antibacterial Agent. Current Drug Delivery, 15(1), 3-20. $\underline{\text { https://doi.org/10.2174/1567201813666160406115744 }}$

Reece, R. J., \& Maxwell, A. (1991). DNA gyrase: Structure and function. Critical Reviews in Biochemistry and Molecular Biology, 26(3-4), 335-375. $\underline{\text { https://doi.org/10.3109/10409239109114072 }}$

Reynolds, M. G. (2000). Compensatory evolution in rifampin-resistant Escherichia coli. Genetics, 156(4), 1471-1481. https://www.ncbi.nlm.nih.gov/pubmed/11102350 
Robicsek, A., Jacoby, G. A., \& Hooper, D. C. (2006a). The worldwide emergence of plasmid-mediated quinolone resistance. The Lancet Infectious Diseases, 6(10), 629-640. https://doi.org/10.1016/S1473-3099(06)70599-0

Robicsek, A., Strahilevitz, J., Jacoby, G. A., Macielag, M., Abbanat, D., Hye Park, C., ... Hooper, D. C. (2006b). Fluoroquinolone-modifying enzyme: A new adaptation of a common aminoglycoside acetyltransferase. Nature Medicine, 12(1), 83-88. https://doi.org/10.1038/nm1347

Roca, I., Akova, M., Baquero, F., Carlet, J., Cavaleri, M., Coenen, S., ... Vila, J. (2015). The global threat of antimicrobial resistance: Science for intervention. New Microbes and New Infections, 6, 22-29. https://doi.org/10.1016/j.nmni.2015.02.007

Rodrigo, G., Landrain, T. E., \& Jaramillo, A. (2012). De novo automated design of small RNA circuits for engineering synthetic riboregulation in living cells. Proceedings of the National Academy of Sciences of the United States of America, 109(38), 15271-15276. https://doi.org/10.1073/pnas.1203831109

Rodrigue, A., Quentin, Y., Lazdunski, A., Méjean, V., \& Foglino, M. (2000). Cell signalling by oligosaccharides. Two-component systems in Pseudomonas aeruginosa: Why so many? Trends in Microbiology, 8(11), 498-504. https://doi.org/10.1016/S0966-842X(00)01833-3

Rodríguez-Martínez, J. M., Machuca, J., Cano, M. E., Calvo, J., Martínez-Martínez, L., \& Pascual, A. (2016). Plasmid-mediated quinolone resistance: Two decades on. Drug Resistance Updates, 29, 13-29. https://doi.org/10.1016/j.drup.2016.09.001 
Rodríguez-Verdugo, A., Gaut, B. S., \& Tenaillon, O. (2013). Evolution of Escherichia coli rifampicin resistance in an antibiotic-free environment during thermal stress. BMC Evolutionary Biology, 13(1), 50. https://doi.org/10.1186/1471-2148-13-50

Rosche, W. A., \& Foster, P. L. (2000). Determining mutation rates in bacterial populations. Methods (San Diego, Calif.), 20(1), 4-17. https://doi.org/10.1006/meth.1999.0901

Rosner, J. L., \& Martin, R. G. (2013). Reduction of cellular stress by TolC-dependent efflux pumps in Escherichia coli indicated by BaeSR and CpxARP activation of spy in efflux mutants. Journal of Bacteriology, 195(5), 1042-1050. https://doi.org/10.1128/JB.01996-12

Rozen, D. E., McGee, L., Levin, B. R., \& Klugman, K. P. (2007). Fitness costs of fluoroquinolone resistance in Streptococcus pneumoniae. Antimicrobial Agents and Chemotherapy, 51(2), 412-416. https://doi.org/10.1128/AAC.01161-06

Ruiz, J. (2003). Mechanisms of resistance to quinolones: target alterations, decreased accumulation and DNA gyrase protection. Journal of Antimicrobial Chemotherapy, 51(5), 1109-1117. https://doi.org/10.1093/jac/dkg222

Ruiz, N., \& Silhavy, T. J. (2005). Sensing external stress: Watchdogs of the Escherichia coli cell envelope. Current Opinion in Microbiology, 8(2), 122-126. $\underline{\text { https://doi.org/10.1016/j.mib.2005.02.013 }}$ 
Sahota, J. S., Smith, C. M., Radhakrishnan, P., Winstanley, C., Goderdzishvili, M., Chanishvili, N., .. Clokie, M. R. J. (2015). Bacteriophage delivery by nebulization and efficacy against phenotypically diverse Pseudomonas aeruginosa from cystic fibrosis patients. Journal of Aerosol Medicine and Pulmonary Drug Delivery, 28(5), 353-360. https://doi.org/10.1089/jamp.2014.1172

Salazar, M. E., \& Laub, M. T. (2015). Temporal and evolutionary dynamics of twocomponent signaling pathways. Current Opinion in Microbiology, 24, 7-14. https://doi.org/10.1016/j.mib.2014.12.003

Saltikov, C. W., \& Newman, D. K. (2003). Genetic identification of a respiratory arsenate reductase. Proceedings of the National Academy of Sciences of the United States of America, 100(19), 10983-10988.

https://doi.org/10.1073/pnas.1834303100

Sander, P., Springer, B., Prammananan, T., Sturmfels, A., Kappler, M., Pletschette, M., \& Böttger, E. C. (2002). Fitness cost of chromosomal drug resistance-conferring mutations. Antimicrobial Agents and Chemotherapy, 46(5), 1204-1211. https://doi.org/10.1128/aac.46.5.1204-1211.2002

Sanders, C. C. (2001). Mechanisms responsible for cross-resistance and dichotomous resistance among the quinolones. Clinical Infectious Diseases, 32(Supplement_1), S1-S8. https://doi.org/10.1086/319369 
Santos-Beneit, F. (2015). The Pho regulon: A huge regulatory network in bacteria.

Frontiers in Microbiology, 6, 402.

https://dx.doi.org/10.3389\%2Ffmicb.2015.00402

Sarkar, S., Ma, W. T., \& Sandri, G. v. H. (1992). On fluctuation analysis: A new, simple and efficient method for computing the expected number of mutants. Genetica, 85(2), 173-179. https://doi.org/10.1007/BF00120324

Schlabach, M. R., Luo, J., Solimini, N. L., Hu, G., Xu, Q., Li, M. Z., ... Elledge, S. J. (2008). Cancer proliferation gene discovery through functional genomics. Science (New York, N.Y.), 319(5863), 620-624. https://doi.org/10.1126/science.1149200

Schoeffler, A. J., \& Berger, J. M. (2008). DNA topoisomerases: Harnessing and constraining energy to govern chromosome topology. Quarterly Reviews of Biophysics, 41(1), 41-101. https://doi.org/10.1017/S003358350800468X

Scholl, C., Fröhling, S., Dunn, I. F., Schinzel, A. C., Barbie, D. A., Kim, S. Y., ... Gilliland, D. G. (2009). Synthetic lethal interaction between oncogenic KRAS dependency and STK33 suppression in human cancer cells. Cell, 137(5), 821-834. https://doi.org/10.1016/j.cell.2009.03.017

Schrag, S. J., Véronique, P., \& Levin, B. R. (1997). Adaptation to the fitness costs of antibiotic resistance in Escherichia coli. Proceedings of the Royal Society of London. Series B: Biological Sciences, 264(1386), 1287-1291. https://doi.org/10.1098/rspb.1997.0178 
Sengupta, S., Chattopadhyay, M. K., \& Grossart, H.-P. (2013). The multifaceted roles of antibiotics and antibiotic resistance in nature. Frontiers in Microbiology, 4, 47. https://doi.org/10.3389/fmicb.2013.00047

Sharma, C. M., \& Vogel, J. (2009). Experimental approaches for the discovery and characterization of regulatory small RNA. Current Opinion in Microbiology, 12(5), 536-546. https://doi.org/10.1016/j.mib.2009.07.006

Sharma, V., Sakai, Y., Smythe, K. A., \& Yokobayashi, Y. (2013). Knockdown of recA gene expression by artificial small RNAs in Escherichia coli. Biochemical and Biophysical Research Communications, 430(1), 256-259.

https://doi.org/10.1016/j.bbrc.2012.10.141

Sharma, V., Yamamura, A., \& Yokobayashi, Y. (2012). Engineering artificial small RNAs for conditional gene silencing in Escherichia coli. ACS Synthetic Biology, 1(1), 6-13. https://doi.org/10.1021/sb200001q

Silva, J. M., Li, M. Z., Chang, K., Ge, W., Golding, M. C., Rickles, R. J., ... Hannon, G. J. (2005). Second-generation shRNA libraries covering the mouse and human genomes. Nature Genetics, 37(11), 1281-1288. https://doi.org/10.1038/ng1650

Silva, J. M., Marran, K., Parker, J. S., Silva, J., Golding, M., Schlabach, M. R., .. Chang, K. (2008). Profiling essential genes in human mammary cells by multiplex RNAi screening. Science (New York, N.Y.), 319(5863), 617-620.

$\underline{\text { https://doi.org/10.1126/science.1149185 }}$ 
Silva, R. F., Mendonça, S. C. M., Carvalho, L. M., Reis, A. M., Gordo, I., Trindade, S., \& Dionisio, F. (2011). Pervasive sign epistasis between conjugative plasmids and drug-resistance chromosomal mutations. PLOS Genetics, 7(7), e1002181. https://doi.org/10.1371/journal.pgen.1002181

Silver, L. L. (2011). Challenges of antibacterial discovery. Clinical Microbiology Reviews, 24(1), 71-109. https://doi.org/10.1128/CMR.00030-10

Siryaporn, A., \& Goulian, M. (2008). Cross-talk suppression between the CpxA-CpxR and EnvZ-OmpR two-component systems in E. coli. Molecular Microbiology, 70(2), 494-506. https://doi.org/10.1111/j.1365-2958.2008.06426.x

Sissi, C., \& Palumbo, M. (2010). In front of and behind the replication fork: Bacterial type IIA topoisomerases. Cellular and Molecular Life Sciences, 67(12), 20012024. https://doi.org/10.1007/s00018-010-0299-5

Skerker, J. M., Perchuk, B. S., Siryaporn, A., Lubin, E. A., Ashenberg, O., Goulian, M., \& Laub, M. T. (2008). Rewiring the specificity of two-component signal transduction systems. Cell, 133(6), 1043-1054.

https://doi.org/10.1016/j.cell.2008.04.040

Smillie, C., Garcillán-Barcia, M. P., Francia, M. V., Rocha, E. P. C., \& de la Cruz, F. (2010). Mobility of plasmids. Microbiology and Molecular Biology Reviews : MMBR, 74(3), 434-452. https://doi.org/10.1128/MMBR.00020-10 
Smith, C. A., \& E. N. Baker. (2002). Aminoglycoside antibiotic resistance by enzymatic deactivation. Current Drug Targets Infectious Disorders 2(2), 143-160. http://dx.doi.org/10.2174/1568005023342533

Smith, C., Heyne, S., Richter, A. S., Will, S., \& Backofen, R. (2010). Freiburg RNA Tools: A web server integrating INTARNA, EXPARNA and LOCARNA. Nucleic Acids Research, 38(Web Server issue), W373-W377. https://doi.org/10.1093/nar/gkq316

Spellberg, B., Guidos, R., Gilbert, D., Bradley, J., Boucher, H. W., Scheld, W. M., ... America, the I. D. S. of. (2008). The epidemic of antibiotic-resistant infections: A call to action for the medical community from the infectious diseases society of America. Clinical Infectious Diseases, 46(2), 155-164. https://doi.org/10.1086/524891

Spellberg, B., Powers, J. H., Brass, E. P., Miller, L. G., \& Edwards Jr., J. E. (2004). Trends in antimicrobial drug development: Implications for the future. Clinical Infectious Diseases, 38(9), 1279-1286. https://doi.org/10.1086/420937

Stanger, F. V, Dehio, C., \& Schirmer, T. (2014). Structure of the N-terminal gyrase B fragment in complex with ADP.Pi reveals rigid-body motion induced by ATP hydrolysis. PLOS One, 9(9), e107289. https://doi.org/10.1371/journal.pone.0107289

Stephenson, K., \& Hoch, J. A. (2002). Virulence- and antibiotic resistance-associated two-component signal transduction systems of Gram-positive pathogenic bacteria 
as targets for antimicrobial therapy. Pharmacology \& Therapeutics, 93(2), 293305. https://doi.org/10.1016/S0163-7258(02)00198-5

Stock, A. M., Robinson, V. L., \& Goudreau, P. N. (2000). Two-component signal transduction. Annual Review of Biochemistry, 69(1), 183-215. https://doi.org/10.1146/annurev.biochem.69.1.183

Stock, J. B., Ninfa, A. J., \& Stock, A. M. (1989). Protein phosphorylation and regulation of adaptive responses in bacteria. Microbiological Reviews, 53(4), 450-490. https://www.ncbi.nlm.nih.gov/pubmed/2556636

Stokes, H. W., \& Gillings, M. R. (2011). Gene flow, mobile genetic elements and the recruitment of antibiotic resistance genes into Gram-negative pathogens. FEMS Microbiology Reviews, 35(5), 790-819. https://doi.org/10.1111/j.1574$\underline{6976.2011 .00273 . x}$

Storz, G., Opdyke, J. A., \& Zhang, A. (2004). Controlling mRNA stability and translation with small, noncoding RNAs. Current Opinion in Microbiology, 7(2), 140-144. https://doi.org/10.1016/j.mib.2004.02.015

Sulavik, M. C., Gambino, L. F., \& Miller, P. F. (1995). The MarR repressor of the multiple antibiotic resistance (mar) operon in Escherichia coli: Prototypic member of a family of bacterial regulatory proteins involved in sensing phenolic compounds. Molecular Medicine (Cambridge, Mass.), 1(4), 436-446. https://www.ncbi.nlm.nih.gov/pubmed/8521301 
Sullivan, E. A., Palumbo, L., Ebrahimzadeh, A., Frieden, T. R., Sullivan, E. A., Frieden, T. R., ... Musser, J. M. (1995). Emergence of fluoroquinolone-resistant tuberculosis in New York City. The Lancet, 345(8958), 1148-1150. https://doi.org/10.5555/uri:pii:S014067369590980X

Sundqvist, M., Geli, P., Andersson, D. I., Sjölund-Karlsson, M., Runehagen, A., Cars, H., ... Kahlmeter, G. (2009). Little evidence for reversibility of trimethoprim resistance after a drastic reduction in trimethoprim use. Journal of Antimicrobial Chemotherapy, 65(2), 350-360. https://doi.org/10.1093/jac/dkp387

Surmann, K., Ćudić, E., Hammer, E., \& Hunke, S. (2016). Molecular and proteome analyses highlight the importance of the Cpx envelope stress system for acid stress and cell wall stability in Escherichia coli. MicrobiologyOpen, 5(4), 582-596. https://doi.org/10.1002/mbo3.353

Swick, M. C., Morgan-Linnell, S. K., Carlson, K. M., \& Zechiedrich, L. (2011). Expression of multidrug efflux pump genes acr $A B$-tolC, $m d f A$, and $n o r E$ in Escherichia coli clinical isolates as a function of fluoroquinolone and multidrug resistance. Antimicrobial Agents and Chemotherapy, 55(2), 921-924. https://doi.org/10.1128/AAC.00996-10

Tamae, C., Liu, A., Kim, K., Sitz, D., Hong, J., Becket, E., ... Miller, J. H. (2008). Determination of antibiotic hypersensitivity among 4,000 single-gene-knockout mutants of Escherichia coli. Journal of Bacteriology, 190(17), 5981-5988. https://doi.org/10.1128/JB.01982-07 
Tang, K. L., Caffrey, N. P., Nóbrega, D. B., Cork, S. C., Ronksley, P. E., Barkema, H. W., ... Ghali, W. A. (2017). Restricting the use of antibiotics in food-producing animals and its associations with antibiotic resistance in food-producing animals and human beings: a systematic review and meta-analysis. The Lancet Planetary Health, 1(8), e316-e327. https://doi.org/10.1016/S2542-5196(17)30141-9

Tang, M., Shen, X., Frank, E. G., O’Donnell, M., Woodgate, R., \& Goodman, M. F. (1999). UmuD'(2)C is an error-prone DNA polymerase, Escherichia coli pol V. Proceedings of the National Academy of Sciences of the United States of America, 96(16), 8919-8924. https://doi.org/10.1073/pnas.96.16.8919

Tenover, F. C. (2006). Mechanisms of antimicrobial resistance in bacteria. The American Journal of Medicine, 119(6), S3-S10. https://doi.org/10.1016/j.amjmed.2006.03.011

The world bank report. (2018). http://www.worldbank.org/en/news/pressrelease/2016/09/18/by-2050-drug-resistant-infections-could-cause-globaleconomic-damage-on-par-with-2008-financial-crisis

Tillotson, G. S. (1996). Quinolones: Structure-activity relationships and future predictions. Journal of Medical Microbiology, 44(5), 320-324.

https://dx.doi.org/10.1099/00222615-44-5-320

Tjaden, B. (2008). TargetRNA: a tool for predicting targets of small RNA action in bacteria. Nucleic Acids Research, 36(Web Server issue), W109-W113. https://doi.org/10.1093/nar/gkn264 
Tommasi, R., Brown, D. G., Walkup, G. K., Manchester, J. I., \& Miller, A. A. (2015). ESKAPEing the labyrinth of antibacterial discovery. Nature Reviews Drug Discovery, 14, 529-542. https://doi.org/10.1038/nrd4572

Toprak, E., Veres, A., Michel, J.-B., Chait, R., Hartl, D. L., \& Kishony, R. (2011). Evolutionary paths to antibiotic resistance under dynamically sustained drug selection. Nature Genetics, 44(1), 101-105. https://doi.org/10.1038/ng.1034

Tran, J. H., Jacoby, G. A., \& Hooper, D. C. (2005). Interaction of the plasmid-encoded quinolone resistance protein Qnr with Escherichia coli DNA gyrase. Antimicrobial Agents and Chemotherapy, 49(1), 118-125. https://doi.org/10.1128/AAC.49.1.118$\underline{125.2005}$

Trindade, S., Sousa, A., Xavier, K. B., Dionisio, F., Ferreira, M. G., \& Gordo, I. (2009). Positive epistasis drives the acquisition of multidrug resistance. PLOS Genetics, 5(7), e1000578. https://doi.org/10.1371/journal.pgen.1000578

UniProt Consortium, T. (2018). UniProt: The universal protein knowledgebase. Nucleic Acids Research, 46(5), 2699. https://doi.org/10.1093/nar/gky092

Urano, H., Yoshida, M., Ogawa, A., Yamamoto, K., Ishihama, A., \& Ogasawara, H. (2017). Cross-regulation between two common ancestral response regulators, HprR and CusR, in Escherichia coli. Microbiology, 163(2), 243-252.

https://dx.doi.org/10.1099/mic.0.000410 
Vales, L. D., Rabin, B. A., \& Chase, J. W. (1982). Subunit structure of Escherichia coli exonuclease VII. Journal of Biological Chemistry, 257(15), 8799-8805. http://www.jbc.org/content/257/15/8799.abstract

Vazquez-Anderson, J., \& Contreras, L. M. (2013). Regulatory RNAs: Charming gene management styles for synthetic biology applications. RNA Biology, 10(12), 17781797. https://doi.org/10.4161/rna.27102

Ventola, C. L. (2015). The antibiotic resistance crisis: part 1: causes and threats. $P \& T$ : A Peer-Reviewed Journal for Formulary Management, 40(4), 277-283. https://www.ncbi.nlm.nih.gov/pubmed/25859123

Vila, J., Ruiz, J., Goñi, P., \& De Anta, M. T. (1996). Detection of mutations in parC in quinolone-resistant clinical isolates of Escherichia coli. Antimicrobial Agents and Chemotherapy, 40(2), 491-493. http://doi.org/10.1128/AAC.40.2.491

Viswanathan, M., Lanjuin, A., \& Lovett, S. T. (1999). Identification of RNase T as a high-copy suppressor of the UV sensitivity associated with single-strand DNA exonuclease deficiency in Escherichia coli. Genetics, 151(3), 929-934. https://www.ncbi.nlm.nih.gov/pubmed/10049912

Viswanathan, V. K. (2014). Off-label abuse of antibiotics by bacteria. Gut Microbes, 5(1), 3-4. https://doi.org/10.4161/gmic. 28027

Vogel, J., \& Luisi, B. F. (2011). Hfq and its constellation of RNA. Nature Reviews. Microbiology, 9(8), 578-589. https://doi.org/10.1038/nrmicro2615 
Vogwill, T., \& MacLean, R. C. (2015). The genetic basis of the fitness costs of antimicrobial resistance: A meta-analysis approach. Evolutionary Applications, 8(3), 284-295. https://doi.org/10.1111/eva.12202

Vogwill, T., Comfort, A. C., Furió, V., \& MacLean, R. C. (2016). Persistence and resistance as complementary bacterial adaptations to antibiotics. Journal of Evolutionary Biology, 29(6), 1223-1233. https://doi.org/10.1111/jeb.12864

Wagner, K., Moolenaar, G., van Noort, J., \& Goosen, N. (2009). Single-molecule analysis reveals two separate DNA-binding domains in the Escherichia coli UvrA dimer. Nucleic Acids Research, 37(6), 1962-1972.

https://doi.org/10.1093/nar/gkp071

Walsh, C. (2000). Molecular mechanisms that confer antibacterial drug resistance. Nature, 406(6797), 775-781. https://doi.org/10.1038/35021219

Walsh, C. (2003). Antibiotics: Actions, origins, resistance. Washington: American Society for Microbiology (ASM), 335.

Wang, D., Huang, X., Chen, J., Mou, Y., \& Qi, Y. (2017). Characterization of a novel qepA3 variant in Enterobacter aerogenes. Journal of Microbiology, Immunology and Infection, 50(2), 254-257. https://doi.org/10.1016/j.jmii.2016.01.001

Watanabe, T. (1963). Infective heredity of multiple drug resistance in bacteria. Bacteriological Reviews, 27(1), 87-115. $\underline{\text { http://mmbr.asm.org/content/27/1/87.abstract }}$ 
Watanabe, T., Igarashi, M., Okajima, T., Ishii, E., Kino, H., Hatano, M., ... Utsumi, R. (2012). Isolation and characterization of signermycin B, an antibiotic that targets the dimerization domain of histidine kinase WalK. Antimicrobial Agents and Chemotherapy, 56(7), 3657-3663. https://doi.org/10.1128/AAC.06467-11

Weatherspoon-Griffin, N., Yang, D., Kong, W., Hua, Z., \& Shi, Y. (2014). The CpxR/CpxA Two-component regulatory system upregulates the multidrug resistance cascade to facilitate Escherichia coli resistance to a model antimicrobial peptide. Journal of Biological Chemistry, 289, 32571-32582.

https://doi.org/10.1074/jbc.M114.565762

Webber, M. A., \& Piddock, L. J. V. (2003). The importance of efflux pumps in bacterial antibiotic resistance. Journal of Antimicrobial Chemotherapy, 51(1), 9-11. https://doi.org/10.1093/jac/dkg050

Wei, C.-F., Tsai, Y.-H., Tsai, S.-H., Lin, C.-S., Chang, C.-J., Lu, C.-C., ... Lai, H.-C. (2017). Cross-talk between bacterial two-component systems drives stepwise regulation of flagellar biosynthesis in swarming development. Biochemical and Biophysical Research Communications, 489(1), 70-75. https://doi.org/10.1016/j.bbrc.2017.05.077

Weigel, L. M., Anderson, G. J., \& Tenover, F. C. (2002). DNA gyrase and topoisomerase IV mutations associated with fluoroquinolone resistance in Proteus mirabilis. Antimicrobial Agents and Chemotherapy, 46(8), 2582-2587. https://doi.org/10.1128/aac.46.8.2582-2587.2002 
Weinreich, D. M., Delaney, N. F., DePristo, M. A., \& Hartl, D. L. (2006). Darwinian evolution can follow only very few mutational paths to fitter proteins. Science, 312(5770), 111-114. https://doi.org/10.1126/science.1123539

Weinreich, D. M., Watson, R. A., \& Chao, L. (2005). Perspective: sign epistasis and genetic constraint on evolutionary trajectories. Evolution, 59(6), 1165-1174. https://doi.org/10.1111/j.0014-3820.2005.tb01768.x

WHO Report (2017). https://www.who.int/antimicrobial-resistance/en/

Wilke, C. O. (2004). The speed of adaptation in large asexual populations. Genetics, 167(4), 2045-2053. https://doi.org/10.1534/genetics.104.027136

Willmott, C. J. R., Critchlow, S. E., Eperon, I. C., \& Maxwell, A. (1994). The complex of DNA gyrase and quinolone drugs with DNA forms a barrier to transcription by RNA polymerase. Journal of Molecular Biology, 242(4), 351-363. https://doi.org/10.1006/jmbi.1994.1586

Winkler, J., \& Kao, K. C. (2012). Harnessing recombination to speed adaptive evolution in Escherichia coli. Metabolic Engineering, 14(5), 487-495. https://doi.org/10.1016/j.ymben.2012.07.004

Wolfson, J. S., \& Hooper, D. C. (1985). The fluoroquinolones: Structures, mechanisms of action and resistance, and spectra of activity in vitro. Antimicrobial Agents and Chemotherapy, 28(4), 581-586. https://doi.org/10.1128/aac.28.4.581 
Wong, A. (2017). Epistasis and the evolution of antimicrobial resistance. Frontiers in Microbiology, 8, 246. https://doi.org/10.3389/fmicb.2017.00246

Wong, A., \& Seguin, K. (2015). Effects of genotype on rates of substitution during experimental evolution. Evolution, 69(7), 1772-1785.

https://doi.org/10.1111/evo.12700

Wong, A., Rodrigue, N., \& Kassen, R. (2012). Genomics of adaptation during experimental evolution of the opportunistic pathogen Pseudomonas aeruginosa. PLOS Genetics, 8(9), e1002928. https://doi.org/10.1371/journal.pgen.1002928

Worthington, R. J., \& Melander, C. (2013). Combination approaches to combat multidrug-resistant bacteria. Trends in Biotechnology, 31(3), 177-184. https://doi.org/10.1016/j.tibtech.2012.12.006

Wright, G. D. (2000). Resisting resistance: New chemical strategies for battling superbugs. Chemistry \& Biology, 7(6), R127-R132. https://doi.org/10.1016/S1074$\underline{5521(00) 00126-5}$

Wright, G. D. (2005). Bacterial resistance to antibiotics: Enzymatic degradation and modification. Advanced Drug Delivery Reviews, 57(10), 1451-1470. https://doi.org/10.1016/j.addr.2005.04.002

Wright, G. D. (2010). Antibiotic resistance in the environment: A link to the clinic? Current Opinion in Microbiology, 13(5), 589-594.

https://doi.org/10.1016/j.mib.2010.08.005 
Wright, G. D. (2016). Antibiotic adjuvants: Rescuing antibiotics from resistance. Trends in Microbiology, 24(11), 862-871. https://doi.org/10.1016/j.tim.2016.06.009

Wright, G. D., \& Poinar, H. (2012). Antibiotic resistance is ancient: Implications for drug discovery. Trends in Microbiology, 20(4), 157-159. https://doi.org/10.1016/j.tim.2012.01.002

Yamamoto, K., Hirao, K., Oshima, T., Aiba, H., Utsumi, R., \& Ishihama, A. (2005). Functional characterization in vitro of all two-component signal transduction systems from Escherichia coli. Journal of Biological Chemistry , 280(2), 14481456. http://doi:10.1074/jbc.M410104200

Yamane, K., Wachino, J.-I., Suzuki, S., Kimura, K., Shibata, N., Kato, H., ... Arakawa, Y. (2007). New plasmid-mediated fluoroquinolone efflux pump, QepA, found in an Escherichia coli clinical isolate. Antimicrobial Agents and Chemotherapy, 51(9), 3354-3360. https://doi.org/10.1128/AAC.00339-07

Yoo, S. M., Na, D., \& Lee, S. Y. (2013). Design and use of synthetic regulatory small RNAs to control gene expression in Escherichia coli. Nature Protocols, 8,1694 1707. https://doi.org/10.1038/nprot.2013.105

Yoshida, H., Bogaki, M., Nakamura, M., Yamanaka, L. M., \& Nakamura, S. (1991). Quinolone resistance-determining region in the DNA gyrase gyrB gene of Escherichia coli. Antimicrobial Agents and Chemotherapy, 35(8), 1647-1650. https://doi.org/10.1128/aac.35.8.1647 
Yoshida, H., Bogaki, M., Nakamura, S., Ubukata, K., \& Konno, M. (1990). Nucleotide sequence and characterization of the Staphylococcus aureus nor A gene, which confers resistance to quinolones. Journal of Bacteriology, 172(12), 6942-6949. https://doi.org/10.1128/jb.172.12.6942-6949.1990

Yoshida, T., Qin, L., Egger, L. A., \& Inouye, M. (2006). Transcription regulation of ompF and ompC by a single transcription factor, OmpR. Journal of Biological Chemistry, 281(25), 17114-17123. https://doi.org/10.1074/jbc.M602112200

Zhang, A., Wassarman, K. M., Ortega, J., Steven, A. C., \& Storz, G. (2002). The Sm-like Hfq protein increases OxyS RNA interaction with target mRNAs. Molecular Cell, 9(1), 11-22. https://doi.org/10.1016/S1097-2765(01)00437-3

Zhang, L., Fritsch, M., Hammond, L., Landreville, R., Slatculescu, C., Colavita, A., \& Mah, T.-F. (2013). Identification of genes involved in Pseudomonas aeruginosa biofilm-specific resistance to antibiotics. PLOS One, 8(4), e61625.

$\underline{\text { https://doi.org/10.1371/journal.pone.0061625 }}$

Zhang, N., Darbari, V. C., Glyde, R., Zhang, X., \& Buck, M. (2016). The bacterial enhancer-dependent RNA polymerase. The Biochemical Journal, 473(21), 37413753. https://doi.org/10.1042/BCJ20160741C

Zhang, Q., Sahin, O., McDermott, P. F., \& Payot, S. (2006). Fitness of antimicrobialresistant Campylobacter and Salmonella. Microbes and Infection, 8(7), 1972-1978. https://doi.org/10.1016/j.micinf.2005.12.031 
Zhao, X., \& Drlica, K. (2001). Restricting the selection of antibiotic-resistant mutants: A general strategy derived from fluoroquinolone studies. Clinical Infectious Diseases, 33(Supplement_3), S147-S156. https://doi.org/10.1086/321841

Zhao, X., Wang, J. Y., Xu, C., Dong, Y., Zhou, J., Domagala, J., \& Drlica, K. (1998). Killing of Staphylococcus aureus by C-8-methoxy fluoroquinolones. Antimicrobial Agents and Chemotherapy, 42(4), 956-958. https://doi.org/10.1128/AAC.42.4.956

Zheng, Q. (1999). Progress of a half century in the study of the Luria-Delbrück distribution. Mathematical Biosciences, 162(1), 1-32. https://doi.org/10.1016/S0025-5564(99)00045-0

Zhou, L., Lei, X.-H., Bochner, B. R., \& Wanner, B. L. (2003). Phenotype microArray analysis of Escherichia coli $\mathrm{K}-12$ mutants with deletions of all two-component systems. Journal of Bacteriology, 185(16), 4956-4972. https://doi.org/10.1128/JB.185.16.4956-4972.2003

Zschiedrich, C. P., Keidel, V., \& Szurmant, H. (2016). Molecular mechanisms of twocomponent signal transduction. Journal of Molecular Biology, 428(19), 3752-3775. https://doi.org/10.1016/j.jmb.2016.08.003 


\section{Appendix A}

\section{Appendix to Chapter 1}

Table A2.1 Terminology used for the estimation of mutation rates. Based on MSSlikelihood method (Sarkar et al. 1992).

\begin{tabular}{ll}
\hline Term & \multicolumn{1}{c}{ Definition } \\
\hline$m$ & No. of mutational events per culture \\
$\mu$ & Mutation rate; probability of mutation per cell per division or generation \\
$\mathrm{N}$ & Number of cells \\
$\mathrm{N}_{0}$ & Initial number of cells in a culture \\
$\mathrm{N}_{t}$ & Final number of cells in a culture \\
$r$ & Observed number of mutants in a culture \\
$f$ & Mutant fraction or frequency ( $r / \mathrm{N})$ \\
$\mathrm{C}$ & No. of cultures in experiment \\
$p_{0}$ & Proportion of cultures without mutants \\
$p_{r}$ & Proportion of cultures with $\mathrm{r}$ mutants
\end{tabular}


Box A2.1 Equations used in for the estimation of mutation rates (Based on the MSS-

likelihood method).

The values of $\mathrm{N}_{\mathrm{t}}$ and the probability of $m$ were generated by observing $r$ mutants on selective medium, $p_{r}$ (Equation 1).

$$
p_{0}=e^{-m} ; \quad p_{r}=\frac{m}{r} \sum_{i=0}^{r-1} \frac{p_{i}}{(r-i+1)}
$$

Equation 1. Probability function to measure ' $r$ ' mutants; $p_{r}$ to estimate the initial value of ' $m$ '

The value of $m$ was calculated by the multiplying all the $p_{r}$ for each observed value of $r$ and analyzed until the likelihood function reaches its maximum (Equation 2) (Ma et al. 1992). The mutation rate ' $\mathrm{M}$ ' was calculated by following equation, $\mathrm{M}=m / \mathrm{N}_{\mathrm{t}}$ (Sarkar et al. 1992; Hall et al. 2009).

$$
f(r \mid m)=\prod_{i=1}^{c} f\left(r_{i} \mid m\right)
$$

Equation 2. MSS likelihood function with product of $p_{r}$ for each observed value of $r$, among all the parallel cultures. 
Table A2.2 Spontaneous mutation rate, with 95\% confidence interval in E. coli K-12 (MG1655) supplemented with different quinolone antibiotics.

\begin{tabular}{|c|c|c|c|c|c|}
\hline $\begin{array}{l}E . \text { coli K-12 } \\
\text { (MG1655) } \\
\text { with selective } \\
\text { antibiotic }\end{array}$ & $\begin{array}{l}\text { Antibiotic } \\
\text { concentration } \\
(\mu \mathrm{g} / \mathrm{ml}) \text { at } \\
1 \mathrm{XMIC} \text { and } \\
2 \mathrm{XMIC}\end{array}$ & Mutation 'm' & $\begin{array}{l}\text { Mutation } \\
\text { rate }(\mathrm{M}) \text { per } \\
10^{8} \text { cells }\end{array}$ & $\begin{array}{l}95 \% \\
\text { Confidence } \\
\text { interval } \\
\text { (Upper } \\
\text { difference) }\end{array}$ & $\begin{array}{l}95 \% \\
\text { Confidence } \\
\text { interval } \\
\text { (Lower } \\
\text { difference) }\end{array}$ \\
\hline $\begin{array}{l}\text { Nalidixic acid } \\
(1 \mathrm{XMIC})\end{array}$ & 11.11 & 0.368 & 0.76 & 0.49 & 0.39 \\
\hline $\begin{array}{l}\text { Ciprofloxacin } \\
\text { (1XMIC) }\end{array}$ & 0.015 & 1.158 & 2.41 & 1.06 & 0.91 \\
\hline $\begin{array}{l}\text { Ciprofloxacin } \\
(2 \mathrm{XMIC})\end{array}$ & 0.030 & 0.293 & 0.61 & 0.42 & 0.33 \\
\hline $\begin{array}{l}\text { Levofloxacin } \\
\text { (1XMIC) }\end{array}$ & 0.0312 & 1.067 & 2.22 & 1.01 & 0.86 \\
\hline $\begin{array}{l}\text { Levofloxacin } \\
\text { (2XMIC) }\end{array}$ & 0.0625 & 0.174 & 0.36 & 0.29 & 0.22 \\
\hline
\end{tabular}


Table A2.3 MIC fold-change and resistance mutations in $\operatorname{gyr} A$, gyrB, and marR among $56 \mathrm{NAL}^{\mathrm{R}}, \mathrm{CIP}^{\mathrm{R}}$, and $\mathrm{LEVO}^{\mathrm{R}}$ mutants of E. coli $\mathrm{K}-12$ (MG1655).

\begin{tabular}{|c|c|c|c|c|c|c|c|c|}
\hline Mutant & $\begin{array}{l}\text { Resistance } \\
\text { mutations }\end{array}$ & $\begin{array}{l}\text { MIC to } \\
\text { NAL } \\
\text { (fold } \\
\text { change) }\end{array}$ & Mutant & $\begin{array}{l}\text { Resistance } \\
\text { mutations }\end{array}$ & $\begin{array}{l}\text { MIC to } \\
\text { CIP (fold } \\
\text { change) }\end{array}$ & Mutant & $\begin{array}{l}\text { Resistance } \\
\text { mutations }\end{array}$ & $\begin{array}{l}\text { MIC to } \\
\text { LEVO } \\
\text { (fold } \\
\text { change) }\end{array}$ \\
\hline NAL1 & $\mathrm{D} 87 \mathrm{G}(\operatorname{gyr} A)$ & 128 & CIP1 & & 16 & LEVO1 & S83L $(g y r A)$ & 16 \\
\hline NAL2 & S83L $(g y r A)$ & 128 & CIP2 & L509G $(g y r B)$ & 16 & LEVO2 & L33R ( marR) & 16 \\
\hline NAL3 & D426N $(g y r B)$ & 64 & CIP3 & $\mathrm{S} 83 \mathrm{~W}(g y r A)$ & 32 & LEVO3 & $\mathrm{T} 102 \mathrm{~S}(\operatorname{marR})$ & 8 \\
\hline NAL4 & A67S $(g y r A)$ & 64 & CIP4 & $\mathrm{S} 83 \mathrm{~W}(g y r A)$ & 32 & LEVO4 & Q117stop (marR) & 8 \\
\hline NAL5 & S83L $(g y r A)$ & 256 & CIP5 & $\mathrm{D} 87 \mathrm{~N}(g y r A)$ & 32 & LEVO5 & N126fs (marR) & 8 \\
\hline NAL6 & $\mathrm{D} 87 \mathrm{G}(g y r A)$ & 128 & CIP6 & S83L $(g y r A)$ & 64 & LEVO6 & $\mathrm{D} 87 \mathrm{G}(g y r A)$ & 16 \\
\hline NAL7 & S83L $(g y r A)$ & 256 & CIP7 & $\mathrm{H} 281 \mathrm{~L}(g y r B)$ & 32 & LEVO7 & L123S (marR) & 4 \\
\hline NAL8 & S83L $(g y r A)$ & 256 & CIP8 & D87Y (gyrA) & 64 & LEVO8 & E10stop (marR) & 16 \\
\hline NAL9 & S83L $(g y r A)$ & 256 & CIP9 & G81C $(g y r A)$ & 64 & LEVO9 & S83L $(g y r A)$ & 8 \\
\hline NAL10 & A67S $(g y r A)$ & 64 & CIP10 & D87Y $(g y r A)$ & 32 & LEVO10 & G69E (marR) & 8 \\
\hline \multirow[t]{14}{*}{ NAL11 } & $\mathrm{S} 83 \mathrm{~L}(g y r A)$ & 256 & CIP11 & $\mathrm{D} 87 \mathrm{~N}($ gyrA $)$ & 64 & LEVO11 & S83L $(g y r A)$ & 16 \\
\hline & & & CIP12 & S464Y (gyrB) & 32 & LEVO12 & L64fs (marR) & 8 \\
\hline & & & CIP13 & D87Y $(g y r A)$ & 64 & LEVO13 & S83L $(g y r A)$ & 4 \\
\hline & & & CIP14 & G81D $(g y r A)$ & 128 & LEVO14 & Q42E (marR) & 8 \\
\hline & & & CIP15 & & 64 & LEVO15 & S83L $(g y r A)$ & 8 \\
\hline & & & CIP16 & G81D $(g y r A)$ & 128 & LEVO16 & S83L $(g y r A)$ & 8 \\
\hline & & & CIP17 & D87Y $(g y r A)$ & 32 & LEVO17 & S83L (gyrA) & 16 \\
\hline & & & CIP18 & & 64 & LEVO18 & $\begin{array}{l}\text { S83L }(g y r A) \\
\text { D87G, R27P }\end{array}$ & 16 \\
\hline & & & CIP19 & & 64 & LEVO19 & $($ gyrA, marR $)$ & 16 \\
\hline & & & CIP20 & $\mathrm{D} 87 \mathrm{G}(g y r A)$ & 64 & LEVO20 & G81C $(g y r A)$ & 16 \\
\hline & & & CIP21 & S83L $(g y r A)$ & 64 & LEVO21 & & 8 \\
\hline & & & CIP22 & S83L $(g y r A)$ & 128 & & & \\
\hline & & & CIP23 & S83L $(g y r A)$ & 16 & & & \\
\hline & & & CIP24 & & 64 & & & \\
\hline
\end{tabular}




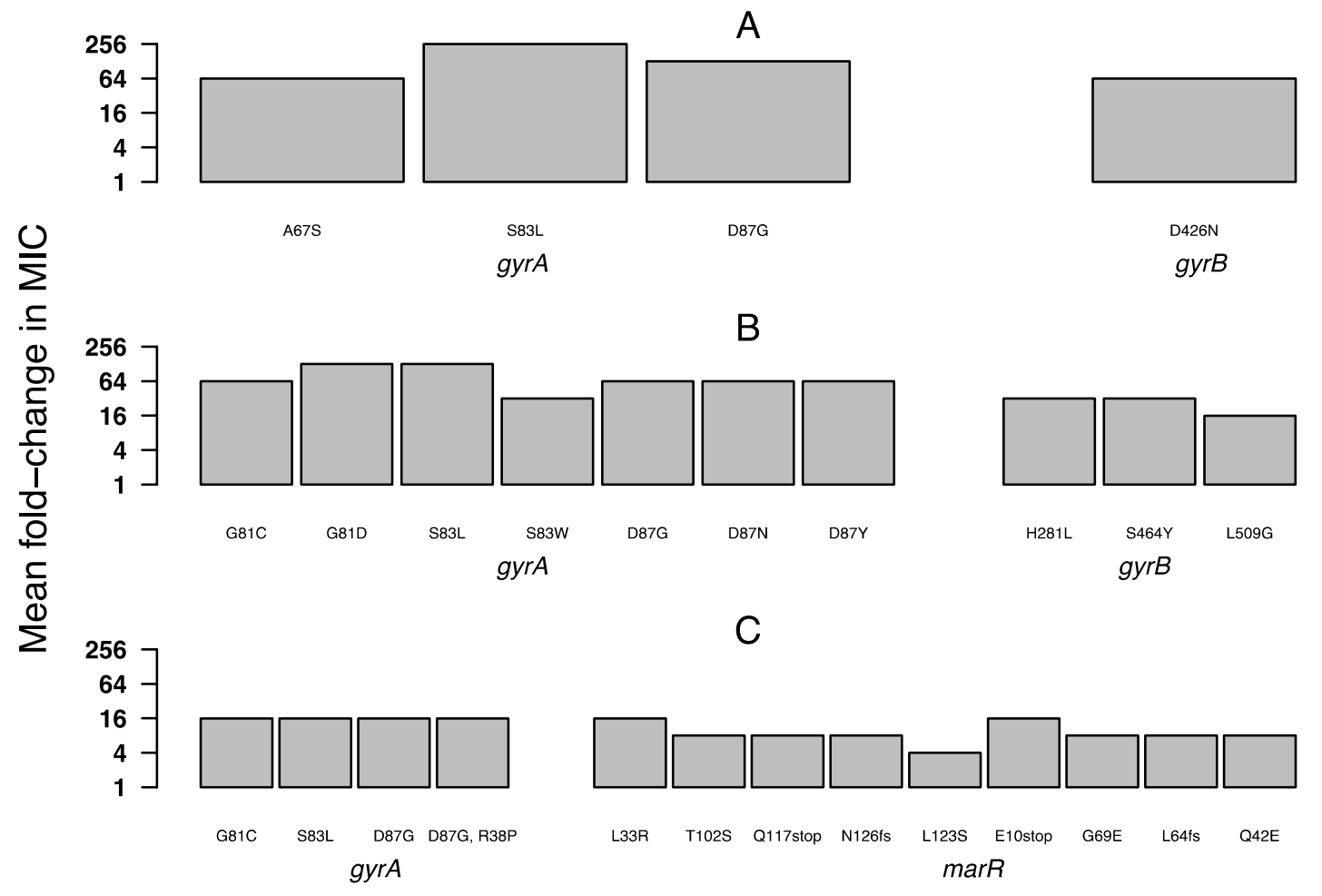

Figure A2.1 Comparison between mean fold-change in MIC values for different resistance mutations in $\operatorname{gyr} A, \operatorname{gyr} B$, and $\operatorname{mar} R$ regions. Resistance mutations isolated as a direct selection on NAL (A), CIP (B), and LEVO (C). 

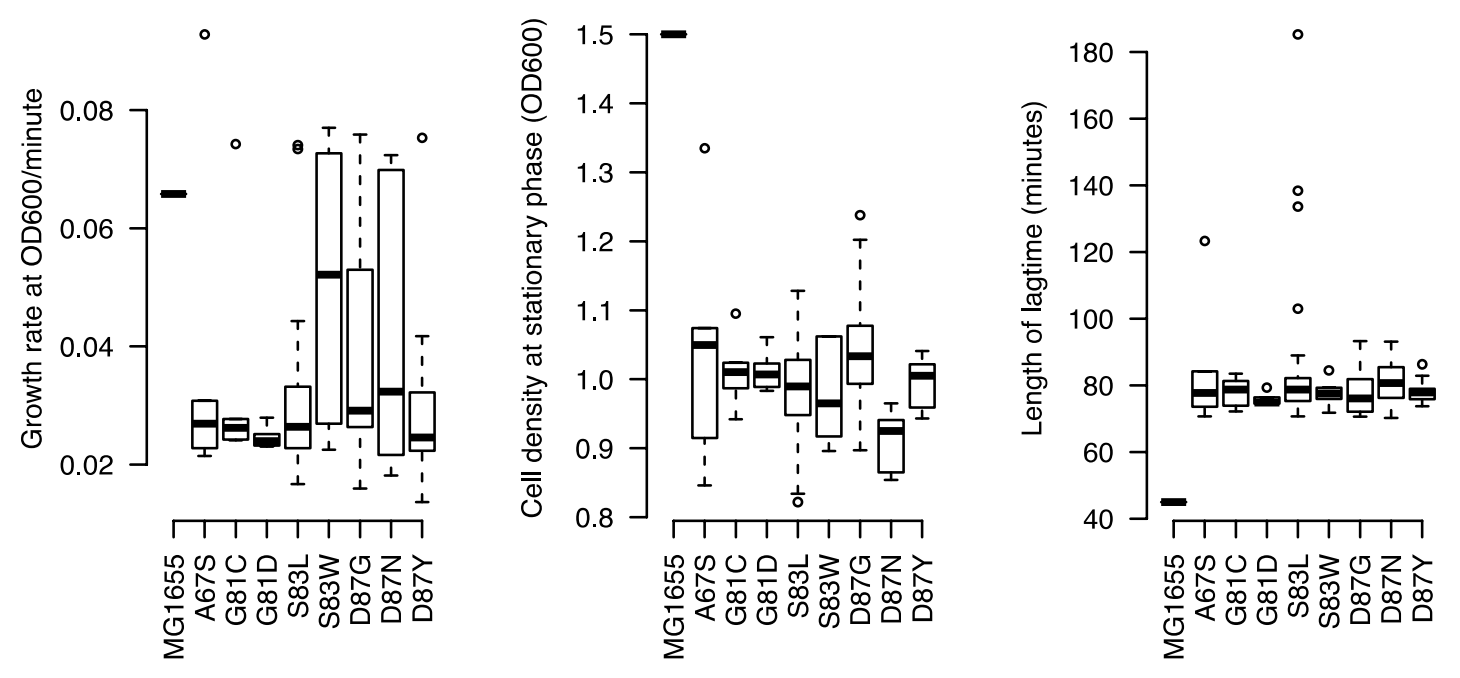

\author{
A. gyr $A$ mutations
}
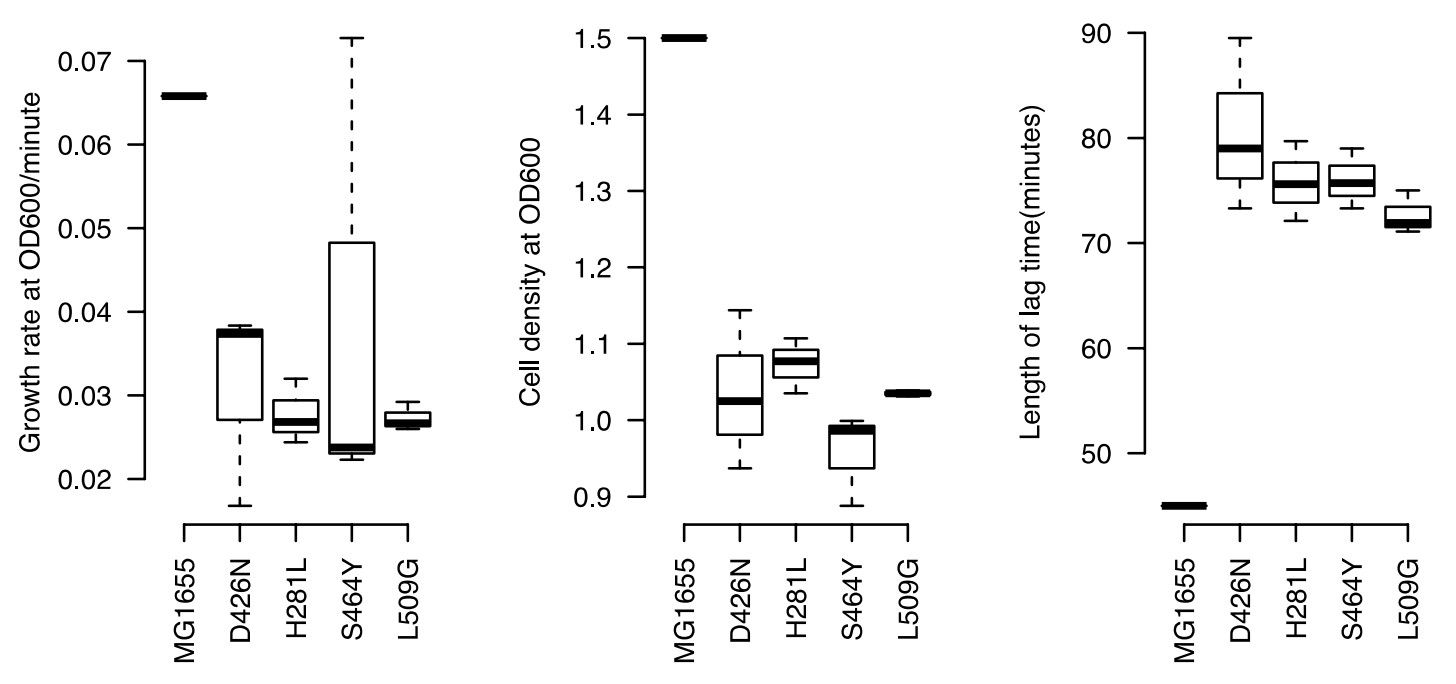

B. $\operatorname{gyr} B$ mutations 

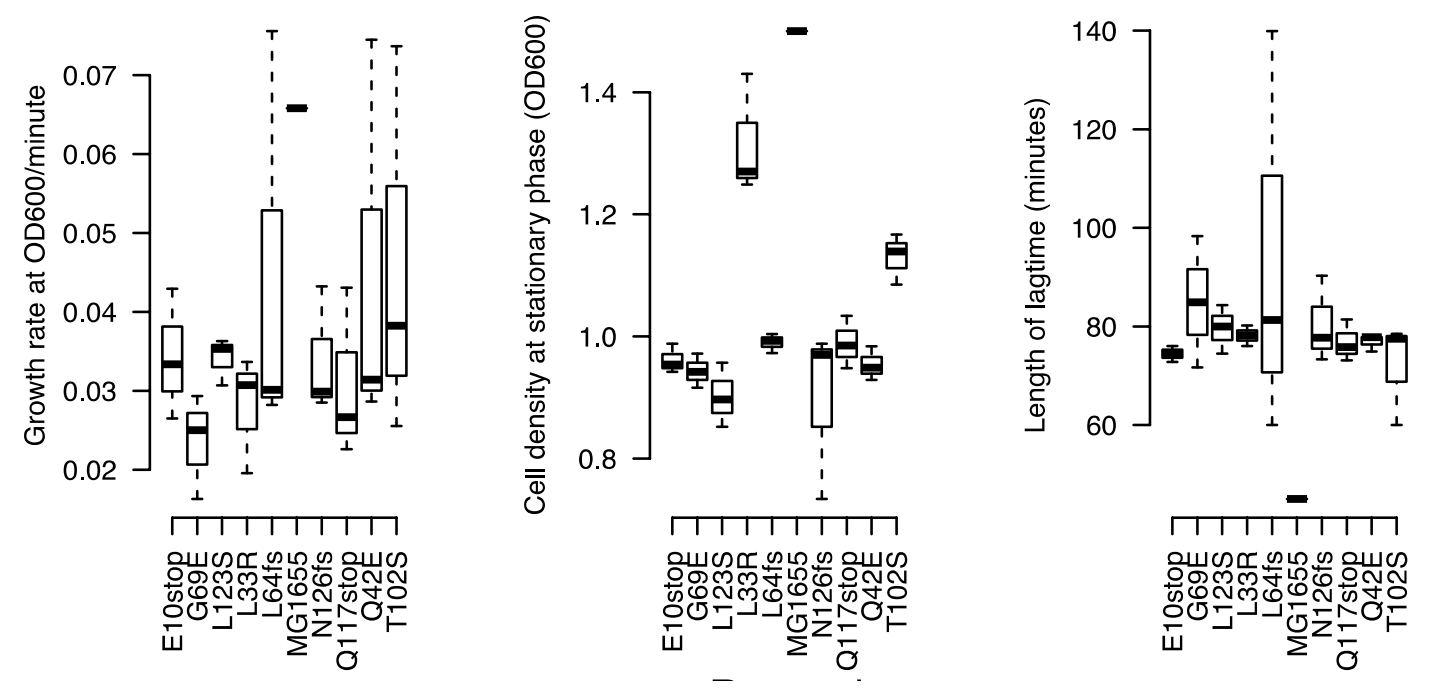

C. $\operatorname{mar} R$ mutations

Figure A2.2 Variation in costs of resistance - growth rate, cell density, and lag phase between $\operatorname{gyr} A(\mathrm{~A}), \operatorname{gyr} B(\mathrm{~B})$, and $\operatorname{marR}(\mathrm{C})$ resistance mutations. All the fitness components are compared to control E. coli K-12 (MG1655). The boxplots present the median, first, and third quartiles, with whiskers showing either the maximum (minimum) value or 1.5 times the interquartile range of the data, whichever is smaller (larger). 


\section{Appendix B}

\section{Appendix to Chapter 2}

Table B3.1 List of oligonucleotides used in this study.

\begin{tabular}{ll}
\hline \multicolumn{1}{c}{ Name } & \multicolumn{1}{c}{ Sequence } \\
\hline pBad_fwd & ATGCCATAGCATTTTTATCC \\
pBad_rev & GATTTAATCTGTATCAGG \\
DsrA_fwd & AATTTTTTAAGTGCTTCTTGCTTAAG \\
DsrA_fwd_randomized & N10-AATTTTTTAAGTGCTTCTTGCTTAAG \\
MicF_fwd & CGTCATTCATTTCTGAATGTCTG \\
MicF_fwd_randomized & N10-CGTCATTCATTTCTGAATGTCTG \\
Spot42_fwd & ATTTGGCTGAATATTTTAGCCGC \\
Spot42_fwd_randomized & N10-ATTTGGCTGAATATTTTAGCCGC \\
Sart_rev & N20-CTCGAGTATGGAGAAACAGTAGAGAG \\
\hline
\end{tabular}


Table B3.2 The list of preliminary (222) gene targets. All the gene targets identified from the 31 sRNA randomized sequences, and their putative mRNA complementarity hits. The hit matches are based on IntaRNA, Target RNA, and RNA predator matches (Mann et al. 2017; Kery et al. 2014; Eggenhofer et al. 2011).

\begin{tabular}{|c|c|c|c|}
\hline S.No & $\begin{array}{l}\text { Native } \\
\text { Scaffold }\end{array}$ & $\begin{array}{l}\text { Randomized } \\
\text { Sequence }\end{array}$ & Target gene hit and their respective function \\
\hline 1 & Dsra & $\begin{array}{l}\text { ttctctcgtcggactgaacgtgga } \\
\text { gctgg }\end{array}$ & $\begin{array}{l}\text { uvrC ATPase and DNA damage recognition protein } \\
\text { of nucleotide excision repair excinuclease UvrABC } \\
\text { exoD Exonuclease V (RecBCD complex); beta } \\
\text { subunit } \\
\text { topB DNA topoisomerase III } \\
\text { emr } A \text { Multidrug efflux system } \\
\text { ampC Penicillin-binding protein beta-lactamase } \\
\text { intrinsically weak } \\
\text { seqA Negative modulator of initiation of replication } \\
\text { tolC Transport channel } \\
\boldsymbol{r p o N} \text { RNA polymerase sigma } 54 \text { (sigma N) factor } \\
\boldsymbol{r e c O} \text { Gap repair protein } \\
\boldsymbol{m i n} C \text { Ftsz ring } \\
\text { umuD Translesion error-prone DNA polymerase V } \\
\text { subunit RecA-activated auto-protease }\end{array}$ \\
\hline 2 & DsrA & $\begin{array}{l}\text { ttgtacctgctttcgatacgactttc } \\
\text { atc }\end{array}$ & $\begin{array}{l}\boldsymbol{m} \boldsymbol{t} \boldsymbol{R} \text { Mannitol operon repressor } \\
\text { tolA Membrane anchored protein in TolA-TolQ-TolR } \\
\text { complex }\end{array}$ \\
\hline 3 & Dsra & $\begin{array}{l}\text { gaattcggaccatgataccactga } \\
\text { gttt }\end{array}$ & 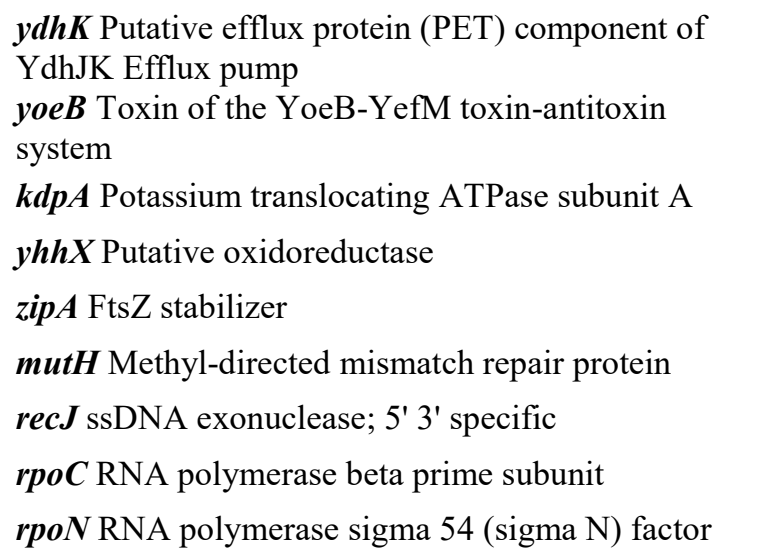 \\
\hline
\end{tabular}


yeeY Predicted DNA binding transcriptional regulator

polA $5^{\prime}$ to $3^{\prime}$ DNA polymerase and $3^{\prime}$ to $5^{\prime} / 5^{\prime}$ to $3^{\prime}$ exonuclease

DsrA gagaccgtgac

ccccacactcccacattcaNNct tgtggg zip $\boldsymbol{A}$ FtsZ stabilizer

helD DNA helicase IV

$\boldsymbol{y} \boldsymbol{g} \boldsymbol{f} \boldsymbol{I}$ Putative DNA-binding transcriptional regulator rpoH RNA polymerase sigma 32 (sigma $\mathrm{H}$ ) factor cho or $y d j Q$ Endonuclease of nucleotide excision repair

dut Deoxyuridinetriphosphatase

rss $\boldsymbol{B}$ PcnB-degradosome interaction factor response regulator

$\boldsymbol{d c u S}$ Sensory histidine kinase in two-component regulatory system with DcuR regulator of anaerobic fumarate respiration

evg $\boldsymbol{A}$ Response regulator in two-component regulatory system with EvgS

$r p o E$ RNA polymerase sigma $\mathrm{E}$ factor

$\boldsymbol{r p o} N$ RNA polymerase sigma 54 (sigma $\mathrm{N}$ ) factor

$\boldsymbol{d n a A}$ Chromosomal replication initiator protein DnaA DNA-binding transcriptional dual regulator $\boldsymbol{r e c} B$ Exonuclease $\mathrm{V}$ (RecBCD complex) beta subunit $\boldsymbol{d} \boldsymbol{k} \boldsymbol{s} \boldsymbol{A}$ DNA binding transcriptional regulator of rRNA transcription; DnaK suppressor protein

recB Exonuclease V (RecBCD complex) beta subunit cheY Chemotaxis regulator transmitting signal to flagellar motor component djla Membrane-anchored DnaK co-chaperone DNAbinding protein

yacG DNA gyrase inhibitor

rec $Q$ ATP-dependent DNA helicase

rpoD RNA polymerase sigma 70 (sigma D) factor

$\boldsymbol{o m p R}$ Response regulator in two-component regulatory system with EnvZ

ити $\boldsymbol{C}$ DNA polymerase $\mathrm{V}$; subunit $\mathrm{C}$

$\boldsymbol{y} \boldsymbol{d a} \boldsymbol{G}$ Putative DNA endonuclease

dnaC DNA biosynthesis protein

recC Exonuclease V (RecBCD complex); gamma chain

holD DNA polymerase III; psi subunit

rpoE RNA polymerase; sigma 24 (sigma E) factor 
$\boldsymbol{d n a A}$ Chromosomal replication initiator protein DnaA DNA-binding transcriptional dual regulator $\boldsymbol{r p o} \boldsymbol{N}$ RNA polymerase sigma 54 (sigma $\mathrm{N}$ ) factor $\min C$ Inhibitor of FtsZ ring polymerization $r p o E$ RNA polymerase sigma $E$ factor recB Exonuclease V (RecBCD complex) beta subunit $r s s B$ PcnB-degradosome interaction factor response regulator

$\boldsymbol{m d t \boldsymbol { A }}$ Multidrug efflux system; subunit A

$\boldsymbol{y p d C}$ Putative DNA-binding protein rho Transcription termination factor $\boldsymbol{y} \boldsymbol{d} \boldsymbol{\boldsymbol { V }} \boldsymbol{\mathrm { R }}$ Rac prophage; predicted DNA replication protein $\boldsymbol{d n a X}$ DNA polymerase III/DNA elongation factor III, tau and gamma subunits

lig $\boldsymbol{A}$ DNA ligase; $\mathrm{NAD}(+)$ dependent $\boldsymbol{d n a B}$ Replicative DNA helicase cgcatt

holE DNA polymerase III theta subunit yacG DNA gyrase inhibitor

$f t s \boldsymbol{Q}$ Division assembly protein; membrane anchored protein involved in growth of wall at septum $\boldsymbol{c p} \boldsymbol{x} \boldsymbol{A}$ Sensory histidine kinase in two component regulatory system with $\mathrm{CpxR}$

11 DsrA acttcattgagacagaacg

$\boldsymbol{y j} \boldsymbol{a} \boldsymbol{A}$ Stress-induced protein

$\boldsymbol{k d p} \boldsymbol{E}$ Response regulator in two-component regulatory system with $\mathrm{KdpD}$

$\boldsymbol{t t d B}$ L-tartrate dehydratase beta subunit $\operatorname{pin} Q$ Qin prophage putative site-specific recombinase

proC Pyrroline-5-carboxylate reductase NAD(P)binding

pepD Aminoacyl-histidine dipeptidase (peptidase D) atoC Fused response regulator of Ato operon in twocomponent system with AtoS: response regulator/sigma54 interaction protein

top $\boldsymbol{A}$ DNA topoisomerase I; omega subunit

hybC Hydrogenase 2 large subunit

12 DsrA caactggtgaa

$\boldsymbol{s m} \boldsymbol{r} \boldsymbol{B}$ Putative DNA endonuclease atoC Fused response regulator of ato operon in twocomponent system with AtoS: response regulator/sigma54 interaction protein

$\boldsymbol{x t h} \boldsymbol{A}$ Exonuclease III 
13

MicF

tacaaccettgccgagaagctagt gttat

tccccttcacgggtgcaacgggc caatgg
15

MicF

16

MicF

tcctcaccegatgtataaaacgtc ccaat

gcagatctccetagatgectagtta ggtg
ypdA Predicted sensory kinase in two component system with $\mathrm{YpdB}$

$\boldsymbol{r} \boldsymbol{b} \boldsymbol{b} \boldsymbol{A}$ Fused ribosome associated ATPase ATP binding protein/ATP binding protein/predicted membrane protein

uvrC ATPase and DNA damage recognition protein of nucleotide excision repair excinuclease UvrABC cho or $y$ dj $Q$ Endonuclease of nucleotide excision repair

$\boldsymbol{z} \boldsymbol{a p B}$ Septal ring assembly factor; stimulates cell division

polA $5^{\prime}$ to $3^{\prime}$ DNA polymerase and $3^{\prime}$ to $5^{\prime} / 5^{\prime}$ to $3^{\prime}$ exonuclease

seqA Negative modulator of initiation of replication

recE Rac prophage exonuclease VIII 5' to 3' specific dsDNA exonuclease

$\boldsymbol{z a p C}$ FtsZ stabilizer

$\boldsymbol{s} \boldsymbol{m} \boldsymbol{f}$ DNA recombination-mediator A family protein

ruvC Component of RuvABC endonuclease

uhpA Response regulator in two-component regulatory system with UhpB

sbmC DNA gyrase inhibitor

$y f j Q$ Uncharacterized protein

yafG $\mathrm{CP} 4-6$ prophage conserved protein

hybC Hydrogenase 2 large subunit

yacG DNA gyrase inhibitor

priA Primosome factor n' (replication factor $\mathrm{Y}$ )

$\min D$ Membrane ATPase of the MinC MinD MinE system

recT Rac prophage; recombination and repair protein rpoD RNA polymerase; sigma 70 (sigma D) factor

$\boldsymbol{d n a E}$ DNA polymerase III alpha subunit

$r p o E$ RNA polymerase sigma $E$ factor

priB Primosomal protein $\mathrm{N}$

zapC FtsZ stabilizer

yhiM Acid resistance protein inner membrane

ftsK DNA translocase at septal ring sorting daughter chromosomes

fts $Y$ Signal Recognition Particle (SRP) receptor 
Multidrug efflux system protein

polB DNA polymerase II

dnaN DNA polymerase III; beta subunit

18

MicF

MicF

19 ctcataaactccgtctccaggatta gggt gacactaaatacgaatgggtggat ttact
recD Exonuclease V (RecBCD complex) alpha chain

$\operatorname{arfA}$ Alternate ribosome-rescue factor $\mathrm{A}$ $\boldsymbol{u} v \boldsymbol{r} \boldsymbol{Y}$ Response regulator in two-component regulatory system with BarA

yneJ Putative DNA-binding transcriptional regulator envY Porin thermoregulatory transcriptional activator

$\boldsymbol{g} \ln \boldsymbol{G}$ Fused DNA binding response regulator in two component regulatory system with GlnL response regulator/sigma54 interaction protein nar $\boldsymbol{X}$ Sensory histidine kinase in two component regulatory system with NarL

recE Rac prophage exonuclease VIII 5' to 3' specific dsDNA exonuclease

rec J 'ssDNA exonuclease; 5' 3' specific

rep DNA helicase and single-stranded DNAdependent ATPase

priB Primosomal protein $\mathrm{N}$

rep DNA helicase and single-stranded DNAdependent ATPase

pyrB Aspartate carbamoyltransferase catalytic subunit

$\boldsymbol{d n a X}$ DNA polymerase III/DNA elongation factor III, tau and gamma subunits

$\boldsymbol{u} \boldsymbol{v r C}$ Excinuclease UvrABC endonuclease subunit

holC DNA polymerase III chi subunit

$\boldsymbol{d n a A}$ Chromosomal replication initiator protein DnaA DNA-binding transcriptional dual regulator $\boldsymbol{u v r B}$ Excision nuclease of nucleotide excision repair DNA damage recognition component

$\boldsymbol{r} \boldsymbol{s} \boldsymbol{g} \boldsymbol{A}$ Ribosome small subunit dependent GTPase A

$\boldsymbol{s b c} \boldsymbol{C}$ Exonuclease; dsDNA; ATP dependent

rep DNA helicase and single-stranded DNAdependent ATPase

$\boldsymbol{c} \boldsymbol{p} \boldsymbol{x} \boldsymbol{A}$ Sensory histidine kinase in two component regulatory system with $\mathrm{CpxR}$

dnaT DNA biosynthesis protein (primosomal protein I)

che $\boldsymbol{A}$ Fused chemotactic sensory histidine kinase in two-component regulatory system with $\mathrm{CheB}$ and CheY: sensory histidine kinase/signal sensing protein

recD Exonuclease V (RecBCD complex) alpha chain 
RNA polymerase beta prime subunit

priC Primosomal replication protein $\mathrm{N} "$

holD DNA polymerase III psi subunit

$\operatorname{din} B$ DNA polymerase IV

sbc $\boldsymbol{C}$ Exonuclease; dsDNA; ATP dependent

recE Rac prophage exonuclease VIII 5' to 3' specific dsDNA exonuclease

zapC FtsZ stabilizer

recE Rac prophage exonuclease VIII 5' to 3' specific dsDNA exonuclease

tus Inhibitor of replication at Ter; DNA binding protein

$\boldsymbol{d n a} \boldsymbol{X}$ DNA polymerase III/DNA elongation factor

III; tau and gamma subunits

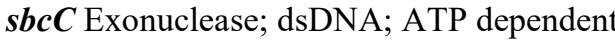

rep DNA helicase and single-stranded DNAdependent ATPase

22 MicF

cagttctttagcagcgcacaggcg tgaca

$\boldsymbol{o m p R}$ Response regulator in two-component regulatory system with EnvZ

$\boldsymbol{y} \boldsymbol{f} \boldsymbol{J} \boldsymbol{J}$ Putative arabinose efflux transporter

rpoB RNA polymerase beta subunit

cheB Fused chemotaxis regulator protein glutamate methylesterase in two component regulatory system with CheA

$\boldsymbol{b a r} \boldsymbol{A}$ Hybrid sensory histidine kinase; in two component regulatory system with UvrY

$\boldsymbol{u} \boldsymbol{v} \boldsymbol{r} \boldsymbol{A}$ ATPase and DNA damage recognition protein of nucleotide excision repair excinuclease UvrABC dnaT DNA biosynthesis protein (primosomal protein I)

torT Periplasmic sensory protein associated with the TorRS two-component regulatory system

recD Exonuclease $\mathrm{V}$ (RecBCD complex) alpha chain

$\operatorname{arc} A$ DNA binding response regulator in two component regulatory system with $\mathrm{ArcB}$ or $\mathrm{CpxA}$ rep DNA helicase and single-stranded DNAdependent ATPase

rnt Ribonuclease $\mathrm{T}$ (RNase $\mathrm{T}$ )

$\boldsymbol{m u t} \boldsymbol{L}$ Methyl directed mismatch repair protein ftsK DNA translocase at septal ring sorting daughter chromosomes I) $\boldsymbol{r e c E}$ Rac prophage exonuclease VIII 5' to 3' specific dsDNA exonuclease 
barA Hybrid sensory histidine kinase; in two component regulatory system with UvrY

$\boldsymbol{r p o B}$ RNA polymerase beta subunit

$\boldsymbol{u v r D}$ DNA-dependent ATPase I and helicase II

dnaT DNA biosynthesis protein (primosomal protein I)

ctaccagaaccgttacgctctaaa caat

gctatcatcattaactttatttattac

MicF

MicF

accctacgaacagtgcaat

MicF

ttaaggaaaaaatgtttNNNNg
tolQ Membrane spanning protein in TolA-TolQ-TolR complex

sapB Antimicrobial peptide transport $\mathrm{ABC}$ transporter permease

$\boldsymbol{d n a} \boldsymbol{Q}$ DNA polymerase III epsilon subunit

rpoZ RNA polymerase omega subunit

$\boldsymbol{f t s} \boldsymbol{A}$ ATP-binding cell division FtsK recruitment protein

torL Response regulator inhibitor for tor operon

$\operatorname{seq} \boldsymbol{A}$ Negative modulator of initiation of replication

$\boldsymbol{o b g} \boldsymbol{E}$ GTPase involved in cell partitioning and DNA repair

$\boldsymbol{y} \boldsymbol{d} \boldsymbol{d} \boldsymbol{M}$ Putative DNA-binding transcriptional regulator

$\boldsymbol{y d j} \boldsymbol{E}$ Putative transporter. putative MFS sugar

$\boldsymbol{r e c} \boldsymbol{A}$ DNA strand exchange and recombination protein with protease and nuclease activity rapA RNA polymerase associated helicase protein (ATPase and RNA polymerase recycling factor) recB exonuclease $\mathrm{V}$ (RecBCD complex) beta subunit $\boldsymbol{d c u S}$ Sensory histidine kinase in two-component regulatory system with DcuR regulator of anaerobic fumarate respiration

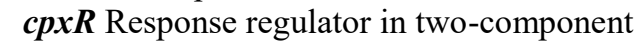
regulatory system with $\mathrm{CpxA}$

yihP Putative 23-dihydroxypropane-1-sulphonate exporter membrane protein

$\boldsymbol{m} \boldsymbol{d t} \boldsymbol{C}$ Multidrug efflux system subunit $\mathrm{C}$

torT Periplasmic sensory protein associated with the TorRS two-component regulatory system

yeh $\boldsymbol{W}$ Putative $\mathrm{ABC}$ transporter permease

$\boldsymbol{r n} \boldsymbol{r}$ Exoribonuclease R; RNase R

$\boldsymbol{u v r} \boldsymbol{B}$ Excision nuclease of nucleotide excision repair DNA damage recognition component

ftsK DNA translocase at septal ring sorting daughter chromosomes

$\boldsymbol{y g} \boldsymbol{b} \boldsymbol{L}$ Putative class II aldolase

cydD Glutathione/cysteine $\mathrm{ABC}$ transporter export permease/ATPase transporter membrane protein 
MicF

ggtagtagactg

29

MicF

ctatacagatcatttacaccc
Spot42

ccgtatagaaccacatctgcetgg ggggg $\boldsymbol{h o l E}$ DNA polymerase III theta subunit

holB DNA polymerase III; delta prime subunit $\operatorname{rap} A$ RNA polymerase associated helicase protein (ATPase and RNA polymerase recycling factor)

hybC Hydrogenase 2 large subunit

priA Primosome factor n' (replication factor Y)

$\boldsymbol{x s e A}$ Exonuclease VII large subunit

topB DNA topoisomerase III

rpoH RNA polymerase sigma 32 (sigma $\mathrm{H}$ ) factor

rрoB RNA polymerase beta subunit

$\boldsymbol{x t h} \boldsymbol{A}$ Exonuclease III

$\boldsymbol{h y} \boldsymbol{b} \boldsymbol{B}$ Predicted hydrogenase 2 cytochrome b type component

rpoH RNA polymerase sigma 32 (sigma $\mathrm{H}$ ) factor

uvrD DNA-dependent ATPase I and helicase II

$\boldsymbol{u v r C}$ Excinuclease UvrABC endonuclease subunit

cpx $\boldsymbol{R}$ Response regulator in two-component regulatory system with $\mathrm{CpxA}$

fic Stationary phase protein; cell division

fts $\boldsymbol{P}$ Septal ring component that protects the divisome from stress; multicopy suppressor of ftsI(Ts)

$\boldsymbol{u v r} \boldsymbol{Y}$ Response regulator in two-component regulatory system with BarA

$\boldsymbol{f t s} \boldsymbol{Q}$ Division assembly protein; membrane anchored protein involved in growth of wall at septum

$\boldsymbol{y} \boldsymbol{h} \boldsymbol{j} \boldsymbol{X}$ Pyruvate-inducible inner membrane protein putative transporter

polA $5^{\prime}$ to $3^{\prime}$ DNA polymerase and $3^{\prime}$ to $5^{\prime} / 5^{\prime}$ to $3^{\prime}$ exonuclease

$\boldsymbol{u v r \boldsymbol { B }}$ Excision nuclease of nucleotide excision repair DNA damage recognition component

dnaT DNA biosynthesis protein (primosomal protein I) umuD Translesion error-prone DNA polymerase $\mathrm{V}$ subunit RecA-activated auto-protease

dnaK Chaperone Hsp70; co chaperone with DnaJ

dnaT DNA biosynthesis protein (primosomal protein I)

torS Hybrid sensory histidine kinase in two component regulatory system with TorR

phoP DNA binding response regulator in two component regulatory system with $\mathrm{PhoQ}$

nar $\boldsymbol{Q}$ Sensory histidine kinase in two component regulatory system with NarP (NarL) 
Table B3.3 The preliminary 74 candidate genes based on their function among 31 artificial sequences of sRNA and their hybridization with target mRNA. Targeted sequencing identified 36/74 mutants (bold) having the gyrA S83L mutation successfully incorporated.

\begin{tabular}{|c|c|c|}
\hline S.NO. & $\begin{array}{c}\text { Target } \\
\text { gene }\end{array}$ & Function \\
\hline 1 & emrA & Multidrug efflux system \\
\hline 2 & fic & Stationary phase protein, cell division \\
\hline 3 & rep & DNA helicase and single-stranded DNA-dependent ATPase \\
\hline 4 & $\operatorname{rec} B$ & Exonuclease V (RecBCD complex) beta subunit \\
\hline 5 & $\operatorname{recC}$ & Exonuclease V (RecBCD complex); gamma chain \\
\hline 6 & recD & Exonuclease V (RecBCD complex) alpha chain \\
\hline 7 & recE & Exonuclease VIII 5' to 3' specific dsDNA exonuclease \\
\hline 8 & recJ & ssDNA exonuclease; 5' 3' specific \\
\hline 9 & $\mathrm{recO}$ & Gap repair protein \\
\hline 10 & $\operatorname{rec} Q$ & ATP-dependent DNA helicase \\
\hline 11 & $\operatorname{rec} T$ & Recombination and repair protein \\
\hline 12 & rho & Transcription termination factor \\
\hline 13 & $r n t$ & Ribonuclease T (RNase T) \\
\hline 14 & rpoC & RNA polymerase beta prime subunit \\
\hline 15 & $r p o D$ & RNA polymerase sigma 70 (sigma $D$ ) factor \\
\hline 16 & $r p o N$ & RNA polymerase sigma 54 (sigma N) factor \\
\hline 17 & $\operatorname{ruvC}$ & Component of RuvABC resolvasome endonuclease \\
\hline 18 & $y a c G$ & DNA gyrase inhibitor \\
\hline 19 & $y p d C$ & Putative DNA-binding protein \\
\hline 20 & $s m r B$ & Putative DNA endonuclease \\
\hline 21 & $\operatorname{smf}$ & DNA recombination-mediator A family protein \\
\hline 22 & $\operatorname{seq} A$ & Negative modulator of initiation of replication \\
\hline 23 & $s b m C$ & DNA gyrase inhibitor \\
\hline 24 & $s b c C$ & Exonuclease; dsDNA; ATP dependent \\
\hline 25 & tus & Inhibitor of replication at Ter; DNA binding protein \\
\hline 26 & $\operatorname{tor} T$ & $\begin{array}{l}\text { Periplasmic sensory protein associated with the TorRS two-component } \\
\text { regulatory system }\end{array}$ \\
\hline
\end{tabular}




\begin{tabular}{|c|c|c|}
\hline 27 & tolA & Membrane anchored protein in TolA-TolQ-TolR complex \\
\hline 28 & tolQ & Membrane spanning protein in TolA-TolQ-TolR complex \\
\hline 29 & tolC & Transport channel \\
\hline 30 & topB & DNA topoisomerase III \\
\hline 31 & ити $C$ & DNA polymerase $V$; subunit $C$ \\
\hline 32 & $u v r D$ & DNA-dependent ATPase I and helicase II \\
\hline 33 & $u v r C$ & Excinuclease UvrABC endonuclease subunit \\
\hline 34 & $x t h A$ & Exonuclease III \\
\hline 35 & $x \operatorname{se} A$ & Exonuclease VII large subunit \\
\hline 36 & ypdA & Predicted sensory kinase in two component system with YpdB \\
\hline 37 & yee $Y$ & Predicted DNA binding transcriptional regulator \\
\hline 38 & $y d a G$ & Putative DNA endonuclease \\
\hline 39 & $y d a V$ & Predicted DNA replication protein \\
\hline 40 & $y d d M$ & Putative DNA-binding transcriptional regulator \\
\hline 41 & $\operatorname{zip} A$ & FtsZ stabilizer \\
\hline 42 & $\operatorname{ampC}$ & Penicillin-binding protein beta-lactamase intrinsically weak \\
\hline 43 & cho & Endonuclease of nucleotide excision repair \\
\hline 44 & cheB & $\begin{array}{l}\text { Fused chemotaxis regulator protein glutamate methylesterase in two } \\
\text { component regulatory system with CheA }\end{array}$ \\
\hline 45 & $\operatorname{cpx} A$ & Sensory histidine kinase in two component regulatory system with $\mathrm{CpxR}$ \\
\hline 46 & $\operatorname{cpx} R$ & Response regulator in two-component regulatory system with $\mathbf{C p x A}$ \\
\hline 47 & $\operatorname{dnaK}$ & Chaperone Hsp70; co chaperone with DnaJ \\
\hline 48 & $\operatorname{dnaQ}$ & DNA polymerase III epsilon subunit \\
\hline 49 & dnaT & DNA biosynthesis protein (primosomal protein I) \\
\hline 50 & $\operatorname{din} B$ & DNA polymerase IV \\
\hline 51 & $e m r B$ & Multidrug efflux system protein \\
\hline 52 & $h y b B$ & Predicted hydrogenase 2 cytochrome b type component \\
\hline 53 & holE & DNA polymerase III theta subunit \\
\hline 54 & holC & DNA polymerase III chi subunit \\
\hline 55 & holD & DNA polymerase III; psi subunit \\
\hline 56 & helD & DNA helicase IV \\
\hline 57 & $h y b C$ & Hydrogenase 2 large subunit \\
\hline 58 & $m d t A$ & Multidrug efflux system; subunit A \\
\hline 59 & $m d t C$ & Multidrug efflux system subunit C \\
\hline 60 & $\min C$ & Inhibitor of FtsZ ring polymerization \\
\hline 61 & $m t l R$ & Mannitol operon repressor \\
\hline 62 & mutL & Methyl directed mismatch repair protein \\
\hline
\end{tabular}




\begin{tabular}{|c|c|c|}
\hline 63 & ompR & Response regulator in two-component regulatory system with EnvZ \\
\hline 64 & $\operatorname{pin} Q$ & Qin prophage putative site-specific recombinase \\
\hline 65 & polB & DNA polymerase II \\
\hline 66 & priA & Primosome factor n' (replication factor Y) \\
\hline 67 & priB & Primosomal protein $\mathbf{N}$ \\
\hline 68 & priC & Primosomal replication protein $\mathrm{N}^{\prime \prime}$ \\
\hline 69 & proC & Pyrroline-5-carboxylate reductase NAD(P)-binding \\
\hline 70 & $\operatorname{pyr} B$ & Aspartate carbamoyltransferase catalytic subunit \\
\hline 71 & $r b b A$ & $\begin{array}{l}\text { Fused ribosome associated ATPase ATP binding protein/ATP binding } \\
\text { protein/predicted membrane protein }\end{array}$ \\
\hline 72 & $r n r$ & Exoribonuclease R; RNase R \\
\hline 73 & $r s g A$ & Ribosome small subunit dependent GTPase A \\
\hline 74 & $u v r A$ & $\begin{array}{l}\text { ATPase and DNA damage recognition protein of nucleotide excision repair } \\
\text { excinuclease UvrABC }\end{array}$ \\
\hline
\end{tabular}


Table B3.4 MIC values (ng/ml) of single (Keio knockout mutant) and double mutant (Keio knockout mutant + gyrA S83L mutation) towards Ciprofloxacin.

\begin{tabular}{|c|c|c|c|c|c|c|}
\hline Target gene & $\begin{array}{c}\text { Keio } \\
\text { knockout } \\
\text { mutant } \\
\text { MIC }\end{array}$ & $\begin{array}{c}\text { Double } \\
\text { mutant } \\
\text { strain MIC } \\
\text { (Keio } \\
\text { knockout } \\
\text { mutant }+ \\
\text { gyr } A \text { S83L } \\
\text { mutation) }\end{array}$ & $\begin{array}{l}\text { Effect of } \\
\text { the Keio } \\
\text { mutation } \\
\text { vs. cybC }\end{array}$ & $\begin{array}{c}\text { Effect on } \\
\text { S83L } \\
\text { background }\end{array}$ & $\begin{array}{c}\text { Effect on } \\
\text { WT } \\
\text { background }\end{array}$ & $\begin{array}{c}\text { Effect on } \\
\text { S83L } \\
\text { background }\end{array}$ \\
\hline$c y b C$ (Control) & 31 & 1000 & 1 & 1 & 0 & 0 \\
\hline $\mathrm{recC}$ & 15 & 500 & 0.5 & 0.5 & -1 & -1 \\
\hline$r e c D$ & 31 & 500 & 1 & 0.5 & 0 & -1 \\
\hline recJ & 31 & 500 & 1 & 0.5 & 0 & -1 \\
\hline rnt & 31 & 500 & 1 & 0.5 & 0 & -1 \\
\hline rpoN & 31 & 1000 & 1 & 1 & 0 & 0 \\
\hline$s m r B$ & 31 & 500 & 1 & 0.5 & 0 & -1 \\
\hline $\operatorname{sbm} C$ & 15 & 125 & 0.5 & 0.125 & -1 & -3 \\
\hline tus & 7.8 & 62.5 & 0.25 & 0.0625 & -2 & -4 \\
\hline top $B$ & 31 & 500 & 1 & 0.5 & 0 & -1 \\
\hline uтис & 31 & 500 & 1 & 0.5 & 0 & -1 \\
\hline$u v r D$ & 15 & 125 & 0.5 & 0.125 & -1 & -3 \\
\hline xseA & 7.8 & 62.5 & 0.25 & 0.0625 & -2 & -4 \\
\hline dnaQ & 31 & 500 & 1 & 0.5 & 0 & -1 \\
\hline helD & 31 & 500 & 1 & 0.5 & 0 & -1 \\
\hline priB & 31 & 500 & 1 & 0.5 & 0 & -1 \\
\hline priC & 31 & 500 & 1 & 0.5 & 0 & -1 \\
\hline$r n r$ & 15 & 125 & 0.5 & 0.125 & -1 & -3 \\
\hline$u v r A$ & 15 & 125 & 0.5 & 0.125 & -1 & -3 \\
\hline yee $Y$ & 31 & 500 & 1 & 0.5 & 0 & -1 \\
\hline$m t l R$ & 31 & 500 & 1 & 0.5 & 0 & -1 \\
\hline$y d a G$ & 31 & 500 & 1 & 0.5 & 0 & -1 \\
\hline$y d a V$ & 31 & 500 & 1 & 0.5 & 0 & -1 \\
\hline ompR & 15 & 500 & 0.5 & 0.5 & -1 & -1 \\
\hline cheB & 15 & 125 & 0.5 & 0.125 & -1 & -3 \\
\hline срхA & 31 & 500 & 1 & 0.5 & 0 & -1 \\
\hline$c p x R$ & 31 & 500 & 1 & 0.5 & 0 & -1 \\
\hline dnaK & 31 & 500 & 1 & 0.5 & 0 & -1 \\
\hline
\end{tabular}




\begin{tabular}{lrrrrrr} 
hybB & 31 & 500 & 1 & 0.5 & 0 & -1 \\
emrB & 31 & 125 & 1 & 0.125 & 0 & -3 \\
tolA & 31 & 500 & 1 & 0.5 & 0 & -1 \\
tolQ & 7.8 & 62.5 & 0.25 & 0.0625 & -2 & -4 \\
tolC & 15 & 62.5 & 0.25 & 0.0625 & -2 & -4 \\
$\min C$ & 15 & 500 & 0.5 & 0.5 & -1 & -1 \\
zipA & 31 & 1000 & 1 & 1 & 0 & 0 \\
proC & 15 & 500 & 0.5 & 0.5 & -1 & -1 \\
pyrB & 31 & 500 & 1 & 0.5 & 0 & -1 \\
\hline
\end{tabular}



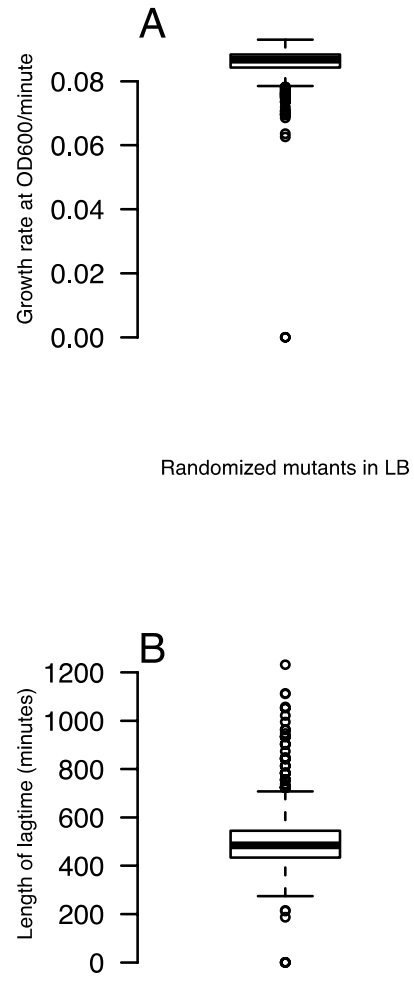

Randomized mutants in LB

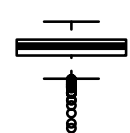

$\circ$

Randomized mutants in LB+CIP

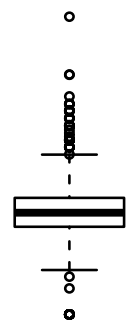

Randomized mutants in LB+CIP

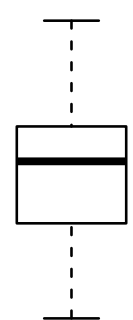

Non-randomized plasmids in LB+CIP

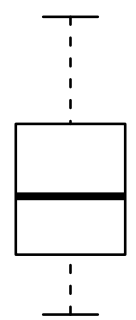

Non-randomized plasmids in LB+CIP

Figure B3.1 A boxplot distribution of 528 randomized plasmid constructs growing in the presence and absence of CIP. The growth parameters tested here are (A) growth rate $\left(\mathrm{OD}_{600} /\right.$ minute) and (B) length of lag phase (minutes). Non-randomized plasmid growing in the presence of CIP is used as a control. The boxplots present the median, first, and third quartiles, with whiskers showing either the maximum (minimum) value or 1.5 times the interquartile range of the data, whichever is smaller (larger). 


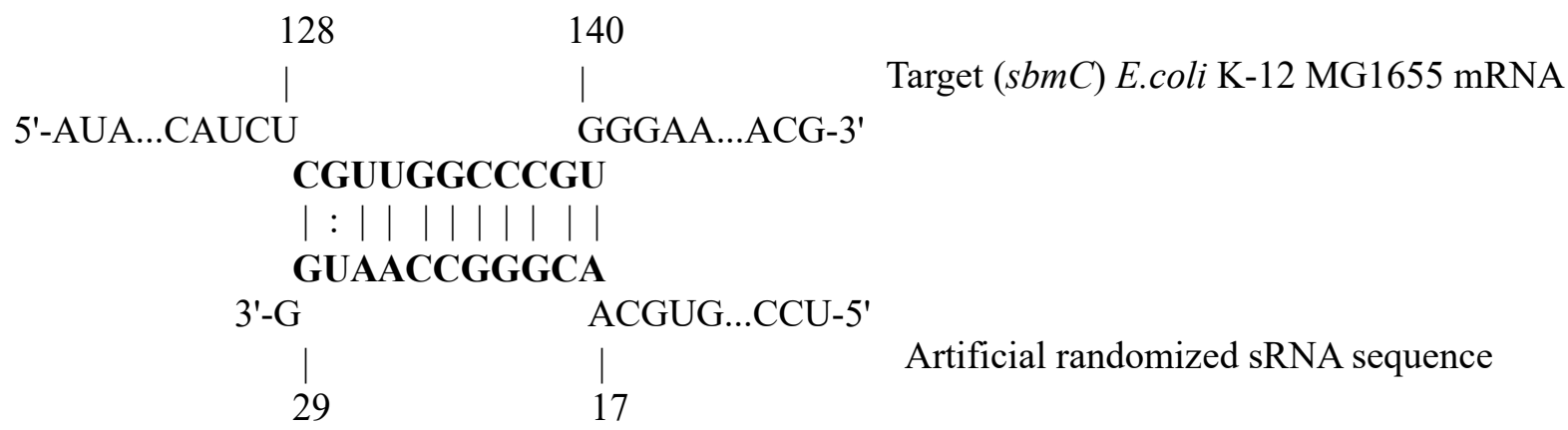

Figure B3.2 A model predicted by IntaRNA, showing the complementary base-pairing between artificial sRNA sequence and its target mRNA hit (Mann et al. 2017). The mRNA target is identified as $s b m C$, which is a DNA gyrase inhibitor gene. The complementary base-pairs and positions are indicated in the figure. 


\section{Appendix C}

\section{Appendix to Chapter 3}

Table C4.1 The list of stressors and their concentrations used in this study.

\begin{tabular}{|c|c|c|c|}
\hline TCS & Stressor & Concentration & References \\
\hline Pho & $\mathrm{MgCl}_{2}$ & $200 \mathrm{uM}$ & $\begin{array}{l}\text { Montero et al. 2009; Ram and Gaulian } \\
2013\end{array}$ \\
\hline $\begin{array}{l}\text { EnvZ- } \\
\text { OmpR }\end{array}$ & $\mathrm{NaCl}$ & $0.5 \mathrm{M}$ & $\begin{array}{l}\text { Mascher 2006; Siryaporn and Goulian } \\
2008\end{array}$ \\
\hline Cpx & $\mathrm{NaCl}$ & $0.5 \mathrm{M}$ & Bury-Moné 2009; Delhaye 2016 \\
\hline Bae & $\mathrm{NaCl}$ & $0.5 \mathrm{M}$ & $\begin{array}{l}\text { Raffa and Raivio, 2002; Baranova } \\
\text { 2002; Bury-Moné } 2009\end{array}$ \\
\hline
\end{tabular}


Table C4.2 The antibiotics used in this study and their mode of action.

\begin{tabular}{llll}
\hline Antibiotic & Class & \multicolumn{1}{c}{ Mode of action } & References \\
\hline Ceftazidime & Third- & Bactericidal. Interfere with bacterial cell & Hayes and \\
& generation & wall synthesis and prevents cross- & Orr 1983 \\
& cephalosporin & linking of peptidoglycan & \\
Ciprofloxacin & Second & Bactericidal. Inhibit enzymes DNA & LeBel 1988 \\
& generation & gyrase and topoisomerase IV, which are & \\
& fluoroquinolone & essential for bacterial DNA replication, & \\
& & recombination, repair, and transcription & \\
Tetracycline & Tetracyclines & Bacteriostatic. Binds reversibly to the & Chopra and \\
& & bacterial 30S ribosomal subunit and & Roberts 2001 \\
& & making the ribosomal site inaccessible & \\
& & for incoming aminoacyl tRNA & \\
& & Bacteriostatic. Interferes with bacterial & Allison et al. \\
& Phenicol & protein synthesis. & 1962 \\
\hline
\end{tabular}


Table C4.3 MIC values (ug/ml) of TCS mutants under stressor +/- antibiotic conditions. (The MIC assay was performed in triplicates with similar results).

\begin{tabular}{|c|c|c|c|c|}
\hline Mutant +/- Stressor & CIP & CEF & TET & СНM \\
\hline$c y b C$-Stressor & 0.015 & 0.06 & 1 & 2 \\
\hline$c y b C+$ stressor $(\mathrm{NaCl})$ & 0.015 & 0.06 & 1 & 2 \\
\hline$c y b C+$ stressor $\left(\mathrm{MgCl}_{2}\right)$ & 0.015 & 0.06 & 1 & 2 \\
\hline phoQ+stressor & 0.015 & 0.125 & 2 & 4 \\
\hline phoQ-stressor & 0.0075 & 0.015 & 0.125 & 0.25 \\
\hline phoP + stressor & 0.031 & 0.125 & 2 & 4 \\
\hline phoP-stressor & 0.0075 & 0.015 & 0.125 & 0.5 \\
\hline env $Z+$ stressor & 0.031 & 0.031 & 0.5 & 0.5 \\
\hline envZ-stressor & 0.0037 & 0.015 & 0.0625 & 0.125 \\
\hline ompR + stressor & 0.031 & 0.125 & 0.5 & 1 \\
\hline ompR-stressor & 0.0037 & 0.015 & 0.0625 & 0.125 \\
\hline$c p x R+$ stressor & 0.015 & 0.25 & 2 & 4 \\
\hline$c p x R$-stressor & 0.0075 & 0.031 & 0.0075 & 0.0075 \\
\hline cpxA+stressor & 0.031 & 0.125 & 1 & 4 \\
\hline cpxA-stressor & 0.0075 & 0.125 & 0.0075 & 0.0075 \\
\hline baeS+stressor & 0.031 & 0.25 & 2 & 4 \\
\hline baeS-stressor & 0.0075 & 0.015 & 0.031 & 0.015 \\
\hline bae $R+$ stressor & 0.031 & 0.5 & 2 & 4 \\
\hline baeR-stressor & 0.0075 & 0.015 & 0.015 & 0.031 \\
\hline
\end{tabular}


Table C4.4 Normalized colony size measurement for eight mutants compared to $c y b C$ on solid media under various experimental treatments. (Each value represents the average measurement of three independently obtained normalized values for each of the mutants).

\begin{tabular}{|c|c|c|c|c|c|c|c|c|c|}
\hline \multirow{2}{*}{ (stressor+/-antibiotic } & \multicolumn{9}{|c|}{ Mutant normalized colony size } \\
\hline & $c y b C$ & baes & baeR & cpxA & cpxR & phoQ & phoP & envZ & ompR \\
\hline M9 MM & 1.96078 & 1.46685 & 1.62367 & 1.50108 & 1.30515 & 1.31109 & 1.54409 & 1.78252 & 1.9344 \\
\hline $\mathrm{M} 9 \mathrm{MM}+\mathrm{NaCl}$ & 1.8848 & 1.35939 & 1.22135 & 1.24019 & 0.96509 & $\mathrm{X}$ & $\mathrm{X}$ & 1.72534 & 1.82534 \\
\hline $\mathrm{M} 9 \mathrm{MM}+\mathrm{MgCl}_{2}$ & 1.86216 & $\mathrm{X}$ & $\mathrm{X}$ & $\mathrm{X}$ & $\mathrm{X}$ & 1.03005 & 1.01651 & $\mathrm{X}$ & $\mathrm{X}$ \\
\hline M9MM+CIP & 1.62013 & 0.02171 & 0.48431 & 0.52069 & 0.7713 & 0.61499 & 0.36078 & 0.89668 & 0.86933 \\
\hline $\mathrm{M} 9 \mathrm{MM}+\mathrm{CEF}$ & 1.7705 & 0.62872 & 0.66601 & 0.96078 & 0.88786 & 0.90878 & 0.81564 & 1.29549 & 1.66537 \\
\hline M9MM+TET & 1.61107 & 0.53866 & 0.37668 & 0.69576 & 0.60208 & 0.43666 & 0.31144 & 1.12696 & 1.15737 \\
\hline M9 MM+CHM & 1.06001 & 0.81899 & 0.85856 & 1.3487 & 0.70793 & 0.95608 & 0.74267 & 1.58049 & 1.96078 \\
\hline $\mathrm{M} 9 \mathrm{MM}+\mathrm{CIP}+\mathrm{NaCl}$ & 1.73427 & 1.0765 & 1.15495 & 1.06656 & 0.87118 & $\mathrm{X}$ & $\mathrm{X}$ & 0.37632 & 0.21836 \\
\hline $\mathrm{M} 9 \mathrm{MM}+\mathrm{CEF}+\mathrm{NaCl}$ & 1.76641 & 1.19183 & 1.32562 & 1.11561 & 1.39772 & $\mathrm{X}$ & $\mathrm{X}$ & 0.67201 & 0.77201 \\
\hline $\mathrm{M} 9 \mathrm{MM}+\mathrm{TET}+\mathrm{NaCl}$ & 1.09607 & 1.27137 & 1.16077 & 1.26457 & 1.06434 & $\mathrm{X}$ & $\mathrm{X}$ & 0.6495 & 0.6495 \\
\hline $\mathrm{M} 9 \mathrm{MM}+\mathrm{CHM}+\mathrm{NaCl}$ & 1.73609 & 1.07932 & 1.3881 & 1.16078 & 0.91582 & $\mathrm{X}$ & $\mathrm{X}$ & 0.56968 & 0.79239 \\
\hline $\mathrm{M} 9 \mathrm{MM}+\mathrm{CIP}+\mathrm{MgCl}_{2}$ & 1.74177 & $\mathrm{X}$ & $\mathrm{X}$ & $\mathrm{X}$ & $\mathrm{X}$ & 0.41048 & 1.3516 & $\mathrm{X}$ & $\mathrm{X}$ \\
\hline $\mathrm{M} 9 \mathrm{MM}+\mathrm{CEF}+\mathrm{MgCl}_{2}$ & 1.06291 & $\mathrm{X}$ & $\mathrm{X}$ & $\mathrm{X}$ & $\mathrm{X}$ & 1.16078 & 1.23997 & $\mathrm{X}$ & $\mathrm{X}$ \\
\hline $\mathrm{M} 9 \mathrm{MM}+\mathrm{TET}+\mathrm{MgCl}_{2}$ & 0.92565 & $\mathrm{X}$ & $\mathrm{X}$ & $\mathrm{X}$ & $\mathrm{X}$ & 0.78968 & 0.93113 & $\mathrm{X}$ & $\mathrm{X}$ \\
\hline $\mathrm{M} 9 \mathrm{MM}+\mathrm{CHM}+\mathrm{MgCl}_{2}$ & 1.00179 & $\mathrm{X}$ & $\mathrm{X}$ & $\mathrm{X}$ & $\mathrm{X}$ & 0.91726 & 1.08668 & $\mathrm{X}$ & $\mathrm{X}$ \\
\hline
\end{tabular}


Table C4.5 A two-way analysis of variance (ANOVA) for the effects of different treatments between control and mutants.

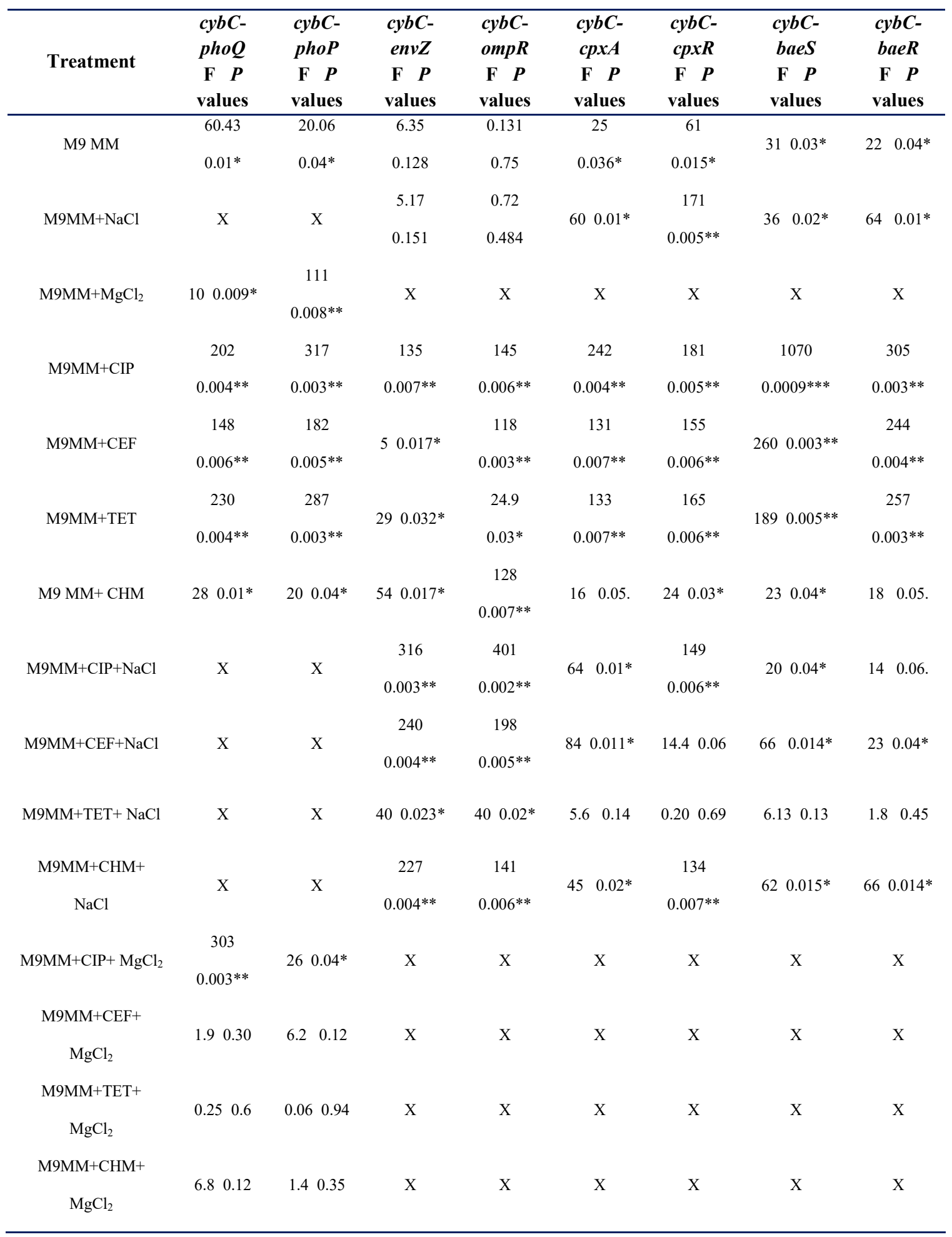




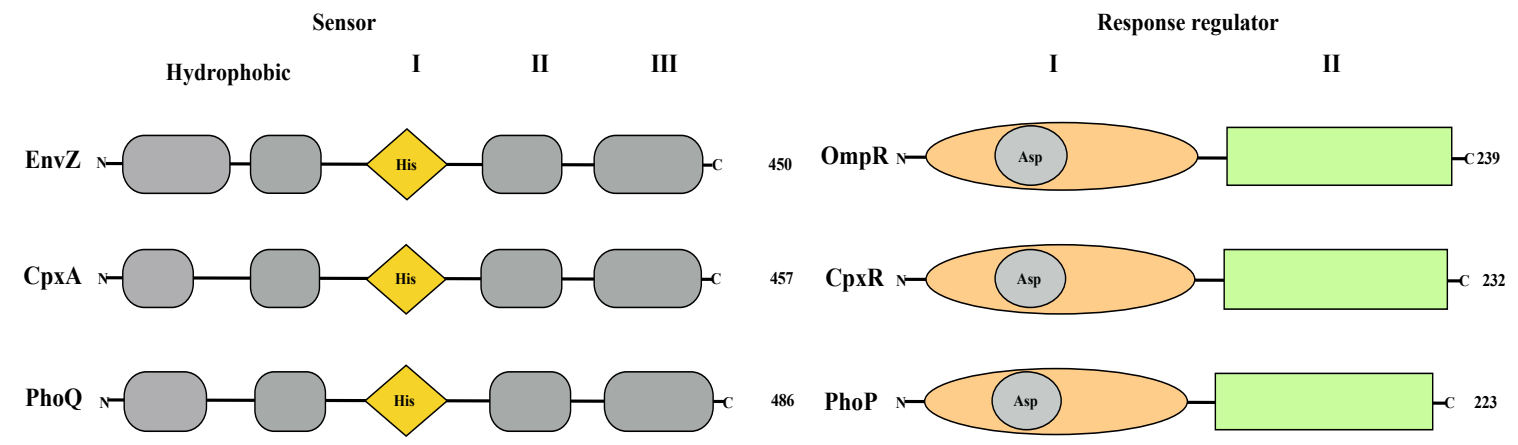

Figure C4.1 Structural features and domain organization of sensor (HK) and response regulator (RR) proteins of E. coli TCS (EnvZ-OmpR, Cpx, and Pho). Region I consist of conserved histidine residue (His), region II is the conserved asparagine, and region III contains glycine-rich residues. The RR consists of N-terminal receiver domain (I) with conserved aspartate (Asp) residue and the other homologous C-terminal output domains (II) for transcriptional regulation. The numbers on the right shows the total number of amino acid residues. (The domain structure and sequences are inspired from Stock et al. 1989; Mizuno et al. 1997; Finn et al. 2016; Keseler et al. 2017) 


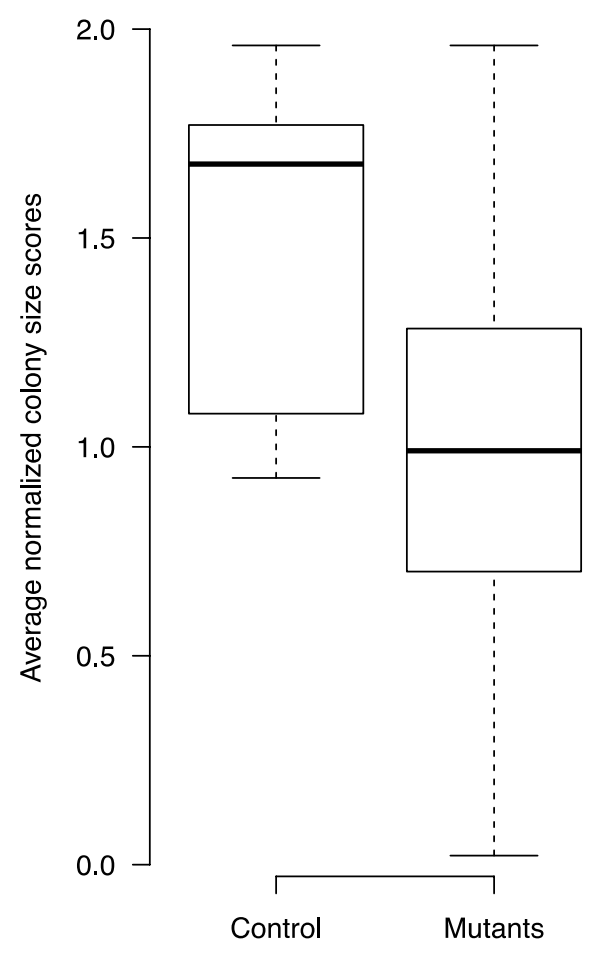

Figure C4.2 Boxplot of averaged normalized colony-size scores comparing various growth treatments (M9 MM and stressor+/-antibiotic) of control $c y b C$ versus eight mutants. The boxplot present the median, first, and third quartiles, with whiskers showing either the maximum (minimum) value or 1.5 times the interquartile range of the data, whichever is smaller (larger). 


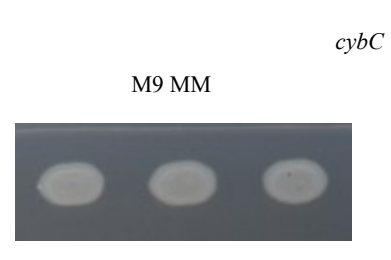

Stressor (-)

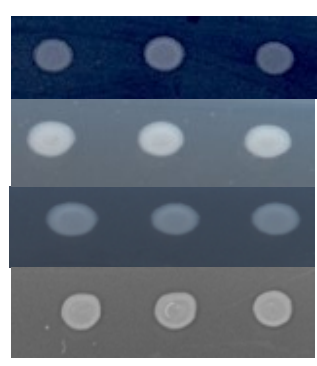

M9MM+Stressor $(\mathrm{NaCl})$

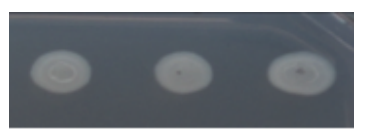

Stressor (+)

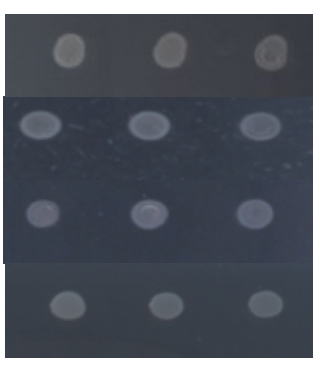

envZ

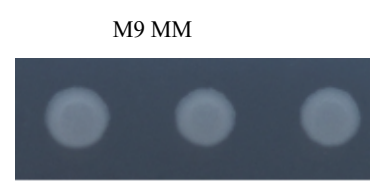

Stressor (-)

Stressor $(+)$

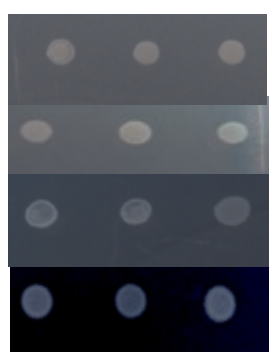

CIP

CEF

TET

CHM

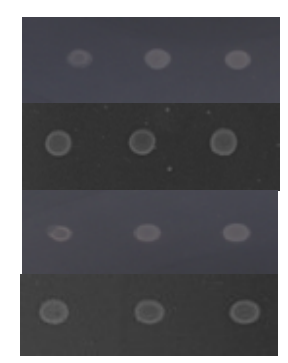

Figure C4.3. A representative image for phenotypic quantification of colony size variation between control $c y b C$ and Keio knockout mutant env $Z$ under various treatments (M9 MM, M9MM+stressor, and stressor +/- antibiotic). 